\title{
Notice
}

DORTEUS OF THIS REPQPT GRE ILLGRBE

LA-8752-MS

4 has been ropraduced from the hest

UC-20f

avallatis copy to fermit the broded

Issued: January 1982

\section{Four-Feedplate Operation of}

\section{ZT-40 with Passive Crowbars}

D. A. Baker

C. J. Buchenauer

L. C. Burkhardt

J. N. Downing

K. B. Freese

R. F. Gribble

A. Haberstich

R. B. Howell
A. R. Jacobson
K. A. Klare
G. Miller
J. A. Phillips
K. S. Thomas
R. G. Watt
P. G. Weber

LA $--8752-$ LS

DE82 008942 
CONTENTS

Abstract $\ldots \ldots \ldots \ldots \ldots \ldots \ldots \ldots \ldots \ldots \ldots \ldots \ldots \ldots \ldots \ldots \ldots \ldots \ldots \ldots \ldots \ldots \ldots 1$

I. Overview of Results--D. A. Baker ........................ 2

II. ZT-40 Description of Operation--K. S. Thonas and J, N. Downing ..... 11

III. Synopsis of operating Diagnostics--R. G. Watt ............... 15

IV. Breakdown Phenomena- R. G. Watt $\ldots \ldots \ldots \ldots \ldots \ldots \ldots \ldots \ldots \ldots \ldots \ldots \ldots \ldots$

V. RF Glow Discharge--L. C. Burkhardt ........................ 20

VI. Start-up Phenomena During Four-Feedplate Operation

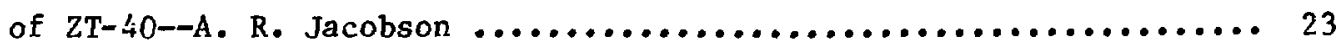

VII. $\mathrm{X}$-Ray and streak observations--L. C. Burkhardt ............... 34

VIII. Alded-Reversal Operation--R. G. Watt ..................... 38

IX. Self-Reversal operation--D. A. Baker ..................... 43

X. Current Decay Times--D. A. Baker ......................... 48

XI. Thomson Scattering Electron Temperature Measurements--A. Haberstich, K. B. Freese, K. A. Klare, and R. G. Watt ................ 58

XII. Basic Features of Electron Density Behavior--A. R. Jacobson ....... 77 XIII. Polarization of Magnetic Fluctuations

at the Wal1--A. R. Jacobsun and C. J. Buchenauer ............ 86

XIV. Pololdal Flux Input as a Function of Toroldal

Current R1setime--J. A. Phill1ps ........................ 95

XV. Measurements of Magnetic Fields in ZT-40--L. C. Burkhardt and J. A. Phillips ................................. 99

XVI. Helical Coil Measurements--G. Miller ...................... 113

XVII. UV Spectroscopic Observations--R. B. Howe11 ................... 119

XVIII. Electron Temperature Estimates From Helium-Like

Ion Line Emisston--P. G. Weber ............................ 135

XIX. Radially Resolved Luminosity Measurements--R. G. Watt ............ 140

XX. Simulations of ZT-40 Current and Voltage Waveforms--R. F. Gribble .. 145 Acknowledgment .................................... 153 
FOUR-FEEDPLATE OPEPATION OF ZT-40 WITH PASSIVE CROWBARS

by

D. A. Baker, C. J. Buchenauer, L. C. Burkhardt,

J. N. Downing, K. B. Freese, R. F. Gribble,

A. Haberstich, R. B. Howell, A. R. Jacobson,

K. A. Klare, G. Miller, J. A. Phillips,

K. S. Thomas, R. G. Watt, and P. G. Weber

\section{ABSTRACT}

This report summarizes the measuremenics made on the ZT-40 reversed-field pinch during the start-up on October 5, 1979, until May 1980, when the power crowbar circultry became operational. The material contained herein pertains to the operation of $2 T-40$ with four current feedpoints, passive crowbars, and a nonconducting, high-purity alumina liner. The plasma properties are given for reversed-fleld pinches obtalned either by aided or self-reversal and for nonreversed-field (stabilized) pinches. It is demonstrated that $\mathrm{ZT}-40$ was capabie of betng ohmically heated to $\sim 100 \mathrm{eV}$ electron temperatures during the $\sim 0.1$-ms rise to peak current. Operation is obtained in which the fluctuations during start-up are observed to decrease later in the discharge, productng quiet periods of $\sim 0.1 \mathrm{~ms}$. 
CHAPTFR I

OVERVIFW OF RESILTS

by

D. A. Baker

\section{TNTRODUCTION}

This report describes the initial results obtained with the operation of ZT-40 using four current feedpoints around the toroidal circumference and a current risetime of $\sim 100 \mu \mathrm{s}$, and with passive crowbar switches on both the toroidal and poloidal field circults. The work of various $2 T-40$ investigators has been divided into separate chapters for each topic.

II. DESCRIPTION OF THE FXPERIMENT

The 2T-40 experiment (Fig. I-1) consists of a high-purity ceramic ( $99.5 \%$ alumina), toroidal vacuum vessel (major radius $=114 \mathrm{~cm}$, minor inside radius = $20 \mathrm{~cm}$, wall thickness $=8 \mathrm{~mm}$ ) surrounded by an aluminum toroldal shell (major radius $=114 \mathrm{~cm}$, minor inside radius $=22 \mathrm{~cm}$, wall thickness $=2.2 \mathrm{~cm}$ ) to provide magnetohydrodynamic (MHD) equilibrium and stability. The vacuum chamber is equipped with sapphire viewing windows and ports for diagnostics and pumping. The details of the segmented construction and arrangements of ports and windows are documented in Refs. 1 and 2. The toroldal fleld is produced by pololdal coils wound on the outside aluminum she11. These coils are energized by a capacitor bank which provides toroidal fields up to $0.5 \mathrm{~T}$, with a risetime of $0.05 \mathrm{~ms}$. The fields are then sustained by a crowbar system. After the toroidal field is energized, the toroldal current is initiated by switching a capacitor bank applying voltage to four feedpoints spaced equally around the toroidal stabilizing shell. The toroldal plasma current is coupled to the toroldal current by 12 equally spaced iron cores (total of $2 \mathrm{~V}-\mathrm{s}$ ) linking the primary and plasma currents. The above arrangement delivers toroldal currents up to $\sim 600 \mathrm{kA}$ rising in $\sim 0.1 \mathrm{~ms}$. The 


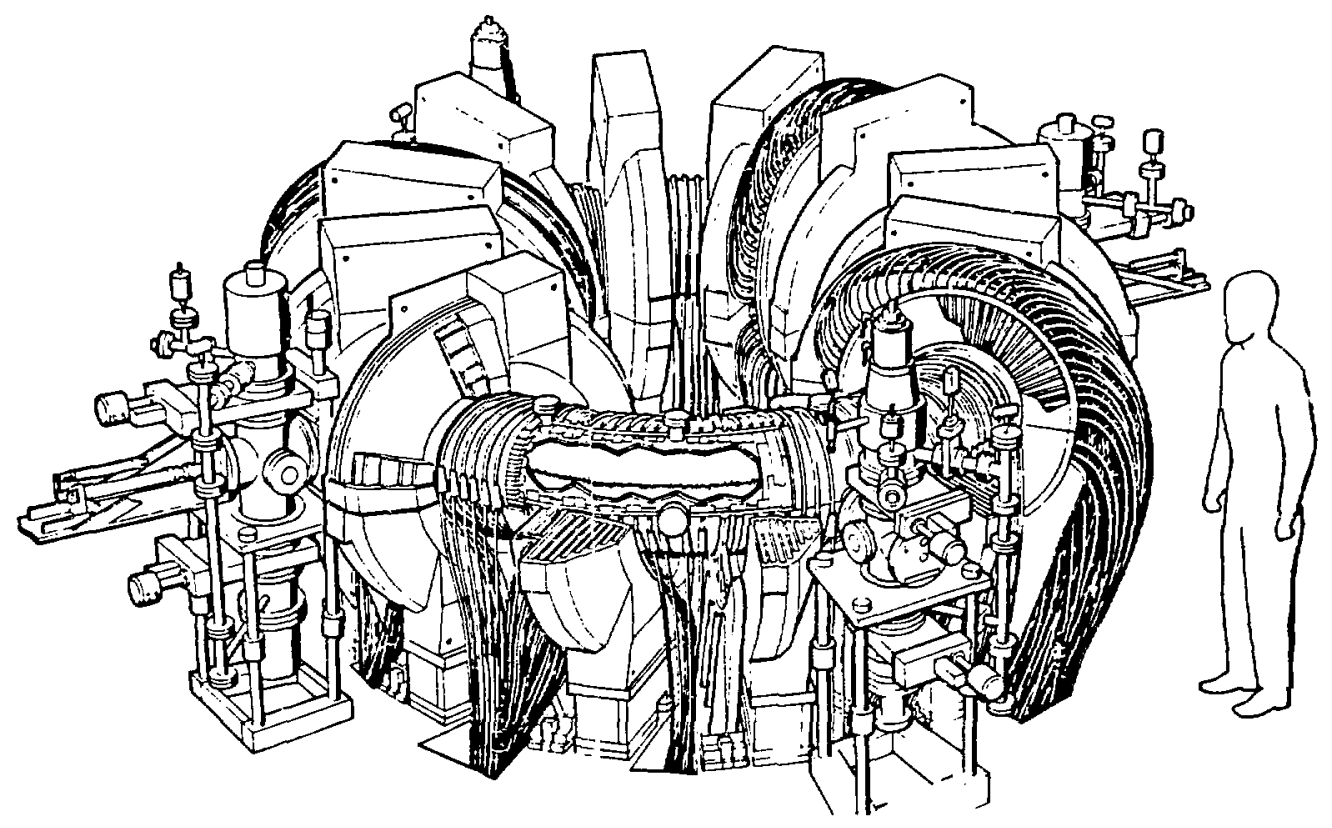

Fig. I-1.

ZT-40 experiment.

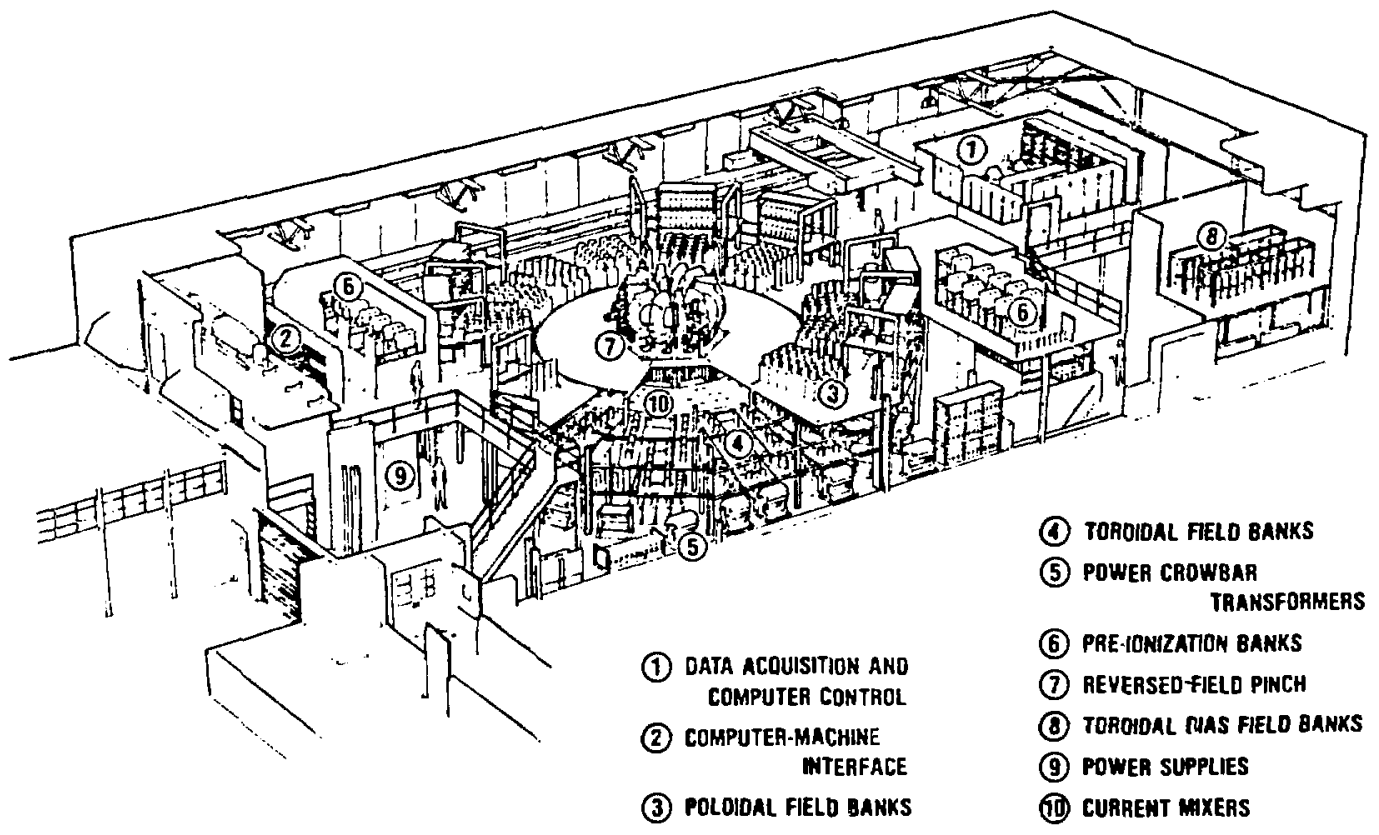

Fig. I-2.

ZT-40 facility. 
details of the energy system are given in Chapter II. An overview of the entire $\mathrm{ZT}-40$ system is shown in Fig. I-2.

The torus is pumped at four equally spaced positions around the major circumference by means of a pair of air bearing, turbomolecular punps and a pair of cryogenic pumps. The base vacuum in the torus reaches $\sim 8 \times 10^{-8}$ mtorr. The initial ionization was assisted, when desired, by a capacitively coupled 27-MHz rf discharge. This rf discharge was also used for glow discharge cleaning. The preionization phase was further implemented by applying a few kiloamperes of toroidal current prior to switching the main. toroidal current capacitor bank.

\section{MODES OF OPERATION}

The $7 T-40$ experiment has been operated as (1) a nonreversed-field pinch (stabilized pinch), (2) a self-reversed pinch, and (3) a reversed-field pinch produced by programming reversed currents in the toroidal-field windings (aided reversa1).

The toroidal current is normally initiated near the time of peak current in the toroidal-field windings. If the toroidal-field windings are crowbarred at this time, the discharge will develop into a reversed or nonreversed pinch configuration depending upon the ratio of the toroidal current to toroidal flux. This ratio is commonly characterized by a dimensionless quantity called the pinch parameter, $\theta=\mathrm{B}_{\theta \mathrm{wal1}} /\left\langle\mathrm{B}_{\phi}\right\rangle$. In $2 \mathrm{~T}-40$ a self-reversal occurs at $\theta \gtrsim 1.5$ or $\geq 1.6$ when $\theta$ is evaluated at the vacuum wall or metal shell, respectively.

The aided-reversal operation is accomplished by delaying the time of crowbar of the toroidal-field circuit, allowing the current to swing through zero and reverse. This mode of operation allows a variety of wavefcrms, depending upon the relative times when the toroidal and poloidal currents are started and their subsequent crowbar switching times.

IV. START-UP

A. Ionization

It was noted that early in the firing history of ZT-40 the main current bank was capable of initiating discharges with deuterium fill pressures down to 2 mtorr. About 800 shots later the lowest unaided breakdown fill pressure was $\sim 5$ mtorr. Lower fill operation then required preionization measures. 
This increasing difficulty of gas breakdown after many shots was attributed to a general discharge cleaning of the vacuum vessel surfaces. The initial preionization was provided by a capacitively coupled $27-\mathrm{MHz}$ rf discharge which was also used for glow discharge cleaning. The preionization was further assisted by applying a few kiloamperes of toroidal current prior to switching the main toroidal current capacitor bank.

B. Density Fluctuations During Start-up

The early time start-up behavior of a stabilized pinch, in which the toroidal bias fie'd is crowbarred (clamped) just prior to the onset of the toroidal current, was studied as a function of fill pressure. It was found that a striking transition in the start-up behavior occurred between fill pressures of 15 and 20 mtorr. Above this transition a multichannel interferometer indicated that the electron density peaked smoothly at the outer minor radius near the wall and this density sheath persisted for the first $\sim 30 \mu \mathrm{s}$ of the current rise. At the lower fill pressures the sheath became unstable and fluctuations in the density appeared. This transition is further evidenced by a marked increase in the early time inductance as deduced from voltage and $\dot{I}$ as the fill pressure was varied between 15 and 20 mtorr. Below the transition fill pressure there was a marked decrease in the subsequent decay rate of the current and an increase in the $x$-ray production presumably by runaway electrons. As discussed later, the deterioration of the decay time of the sticilized pinch was overcome when the discinarge was operated in the reversed-field pinch modes. It was also observed that starting up the current hefore crowbarring the pinch could cause the electron density to peak at the center rather than at the wall.

C. Poloidal Flux Loss During Start-up

The time integral of the toroidal loop voltage was investigated for current risetimes ranging from $0.23 \mathrm{~ms}$ to $0.033 \mathrm{~ms}$. The shorter risetimes were accomplished and obtained by operating at higher than normal capacitor bank voltages and crowbarring the current early during the current rise. The longest risetime, $0.23 \mathrm{~ms}$, was obtained by temporarily operating $\mathrm{ZT}-40$ with one feedplate. The absence of internal magnetic fleld data required the comparison of the measured volt-seconds with assumed model field profiles. This preliminary study indicated that the pololdal volt-second input during start-up was an increasing function of current risetime; the value at peak current for the shortest risetime was about half of that supplied at the 
longest risetime. This effect is believed to be associated with a larger poloidal flux annihilation occurring for longer risetimes. The volt-second input at 3 mtorr fill was ohserved to be about five times the amount at $30 \mathrm{mtorr}$. This correlates with the fluctuation and other phenomena mentioned above and probably is indicative of a larger resistivity at lower fill pressures. The comparison with model field profiles indicates that the poloidal magnetic field has diffused throughout the pinch at the time of peak current for all risetimes.

V. OVERCOMING THE RADIATION BARRIER

One of the first objectives of the $2 T-40$ experiment was to demonstrate that temperatures could be reached well above the 0 VI "radiation barrier" at $\sim 25 \mathrm{eV}$. In earlier small experiments the large line radiation from oxygen impurity atoms clamped the electron temperatures at $\sim 15-25 \mathrm{eV}$ and precluded the ohmic heating of those experiments to higher temperatures. After a number of clean-up discharges, measurements of electron temperature by Thomson scattering showed that $\mathrm{T}_{e}$ on the axis of $\mathrm{ZT}-40$ was reaching $\sim 100 \mathrm{eV}$ by the time of peak current. The electron temperature was therefore not being clamped by the impurities and the radiation barrier had been overcome.

Supporting evidence of the burnthrough of the radiation barrier was given by spectroscopic observations. The oxyger impurity $0 \mathrm{~V}$ and $O$ VI spectral lines were observed to reach a feak and fall as the lines were exclted and stripped. In general, $z$ second peak in the radiation from these states appeared as the temperature fell later in the discharge. Numerical solution of the rate equations for oxygen fonfzation levels and onmic heating indicates that the observed $T_{e} \sim 100 \mathrm{eV}$ is consistent with the observed burnthrough time of O VI. Observations on the helium-like ionization states of nitrogen, carbon, and oxygen also give temperature estimates which support the conclusion that $Z T-40$ has reached eiectron temperatures well above the oxygen radiation barrier.

\section{SUSTAINMENT PHASE}

A. Current Decay Time

It was noted early in the operation of $2 T-40$ that, for nonreversed-field operation, the discharge displayed a marked transition in its behavior as the filling pressure was reduced. Between 15 and 20 mtorr fill a trend began in 
which the current decay rate increased with decreasing fill pressure. This behavior is correlated with the changes in early time inductance, density sheaths, and density fluctuations mentioned in Section IV-B. An 1riteresting feature is that the current decay time markedly improved as the discharge was operated in the alded-reversal mode, converting it into a reversed-field pinch (RFP). The decay time of the current was observed to lengthen as increasing amounts of aided reversal were applied by delaying the time of crowbar of the reversed fleld. These longer decay times are generally interpreted as indicating a lower dissipation and loss rate in the plasma resulting from the better containment properties of the reversed-field configuration. Studies of the current waveforms show that the longest current decay times (0.3-0.4 ms) are being influenced markedly by the external circult resistance. The effective plasma resistance deduced from the calculations for the RFP mode are on the order of $3 \mathrm{~m} \Omega$. This effective resistance is defined to give the total field dissipation (that is, $I_{\phi}{ }^{2} R_{e f f}=\int_{v o l}^{\vec{J}} \cdot \vec{E} d v$ ) and therefore includes energy which may be going into macroscopic motion and turbulence as well as ohmic dissipation.

\section{B. Temperature}

The temperatures on the minor axis of the discharge have been measured for nonreversal, self-reversal, and alded-reversal modes of operation. In the first instance the temperature at peak current was found to be a monotonic decreasing function of fill pressure, dropping from a maximum of $\sim 80 \mathrm{eV}$ at $\sim 5$ mtorr fill to $20-30 \mathrm{eV}$ at $40 \mathrm{mtorr}$. It is notable that temperatures as high as $\sim 30 \mathrm{eV}$ were seen even at high fill pressures, indicating that the Impurity level was low. The measurements indicate that $T_{e}$ at the time of peak current rose nearly linearly with the value of peak current. This deviation from ohmic $I^{2}$ scaling resulted from the loss processes which occurred during the current rise.

The measurements for RFP configurations indicated about the same $T_{e}$ time dependence for both self-reversal and alded-reversal methods of production. For currents of $480 \mathrm{kA}$ the temperatures reached $\sim 100 \mathrm{eV}$ and decayed with the current to $\sim 30 \mathrm{eV}$ at $\sim 0.2 \mathrm{~ms}$ after peak current.

C. Density observations

The observations of the path-averaged electron density along seven vertical chords with a multichord interferometer indicated that the density profile became flattened by the time of peak current. The measurements 
indicated that the total electron inventory measured across the discharge cross section dropped after reaching a maximum near the time of peak current. The inventory reduction generally ranged from $\sim 50 \%$ to $\sim 80 \%$ of the initial fi11, with the larger percent reductions occurring at the lower fill pressures. Similar reductions of electron density during setup were observed recently in Eta Beta II. ${ }^{3}$ Evidently this reduction is associated with the fluctuations accompanying the setting up of the discharge configuration.

D. Magnetic Field Measurements

Internal magnetic probe data taken with the passive crowbar operation was very limited. There is the constant concern that breakage of the probe jacket can result in contamination of the discharge. A special single loop probe was constructed from aluminum and ceramic and encased in a sapphire jacket. These data indicate that there is no pronounced current sheath and that the fields deviate significantly from the Bessel function Taylor states near the walls. E. Observations of Quiet Periods

An important objective of the RFP program is to achieve a configuration in which the transport losses are low enough to allow the temperature to continue to rise due to the ohmic heating from the large current densities that are present. Of particular interest is the observation of a lowfluctuation-level discharge, after the initial turbulence which accompanies the current rise, seen first on $\mathrm{Zeta}^{4}$ and later on Eta Beta II. ${ }^{3}$ These quiet perinds have been reported to be associated with low energy loss rates, relatively high plasma conductivity, and a rising electron temperature. Indications of such a phenomenon on ZT-40 were first seen on a multichannel interferometer which simultancously measures the integral of the electron density along seven vertical chords normal to the midplane of the torus. During self-reversal operation at 4-to 5-mtorr filling pressures, a large reduction in the density fluctuations was observed in about one-third of the discharges. Although the appearance of these "density quiet periods" are not reproducible, it is notable that they only apprared under similar conditions reported for Zeta, namely only during the decay of the toroldal current when $E_{\phi}$ and $B_{\phi}$ at the wall were reversed. The density quiet periods terminate near the time the reversed field was lost. However, a noticeable difference in these quiet periods from zeta and Eta Beta II is that they were not accompanied by large drops in the level of the dI/dt fluctuations. 
In contrast to the above density quiet periods, when $2 \mathrm{~T}-40$. was operated with aided reversal, "electrical quiet perfods" were observed in which large reductions in the fluctuations of toroidal voltage $\mathrm{dI} / \mathrm{dt}$ and signals from ${ }^{\mathrm{B}_{\theta}}$ and $\mathrm{B}_{\phi}$ magnetic pickup loops outside the vacuum torus occurred. Ccrresponding drops in density fluctuations were often observed, but not always. These electrical quiet periods probably represented a condition of lower transport loss because they are normally accompanied by a reduction in the decay rate of the current. These quiet periods also displayed shot-to-shot variations. For fill pressures of $\sim 5$ mtorr they occurred, at best, in about two out of three discharges. The rate of occurrence of these quiet periods varied strongly on plasma parameters and operating conditions. For passive crowbar operation these quiet periods varled in length, lasting $\lesssim 0.1 \mathrm{~ms}$. The electrical quiet periods for $2 \mathrm{~T}-40$ had a much more unpred ctable nature than those observed in Eta BEta II. In that machine, once proper operating conditions were obtained, the duration and occurrence of the quiet periods were very reproducible. The nonreproducibility of these quiet periods in $\mathrm{ZT}-40$ precluded a detalled measurement of ohmic heating during the low -fluctuation period. The most probable cause for the nonreproducibility appears to be associated with the use of a nonmetallic vacuum liner in the ZT-40 experiment. Possible explanations include: (1) a metallic liner in direct contact with the plasma can suppress electrostatic modes, thus reducing the turbulence and transport, (2) the resistive liner has a damping effect in the plasma-circuit system, (3) the ceramic liner may be releasing gas later in the discharge, quenching the temperature.

\section{CONCLUSION}

The $\mathrm{ZT}-40$ experiment has successfully cvercome the oxygen radiation barrier and achieved $\sim 100 \mathrm{eV}$ temperatures and poloidal beta values of $\sim 10 \%$. The grossly stable RFP configurations have been achieved using either self-reversal or aided reversal, and the existence of quiet periods with low fluctuations has been demonstrated. The quiet periods have been limited in duration to $\sim 0.1 \mathrm{~ms}$. The duration and occurrence of the quiet periods are much less reproducible than observed in Eta Beta II. It appears that this difference comes from the use of an insulating vacuum chamber in $\mathrm{ZT}-40$. This hypothesis will be studied when $Z T-40$ comes on-1ine with a metallic vacuum 
liner. The results obtalsed utflizing power crowbar circults to extend the toroldal current and reversed field will be discussed in subsequent reports.

\section{RFFERENCES}

1. W. E. Hauth, R. T. B1ake, J. M. Dickenson, H. L. Rutz, and S. N. Stoddard, "Fabrication of An Alumina Torus for Thermonuclear Fusion Containment," Los Alamos Scientific Laboratory report LA-7255-MS (May 1978).

2. R. S. Dike and A. Haberstich, "Ceramic Toroldal Vacuum Chamber for the ZT-40 Fxperiment," Proc. 8th Symp. on Eng. Problems of Fusion Research, San Francisco, Calffornia, November 13-16, 1979 (IEEF Publication - 79CH1441-5NPS) Vo1. 4, P. 2117.

3. A. Buffa, S. Costa, R. DeAngelis, L. Giudicotti, C. W. Gowers, G. Malesani, G. F. Nalesso, S. Ortolani, P. Scarin, ard M. R. C. Watts, "Reversed-Field Pinch Plasmas With Improved Confinement," Proc. 8th Int. Conf. on Plasma Phys. and Controlled Nucl. Fusion Research, Brussels, Belgium, July 1-10, 1980 (IAEA, Vienna, Nuclear Fusion, Supplement 1981 Publication) Vo1. 2 , p. 275.

4. H. A. B. Bodin and A. A. Newton, Nucl. Fusion 20, 1255 (1980). 
CHAPTER II

ZT-40 DESCRIP'IION OF OPERATION

by

K. S. Thomas and J. N. Downing

This section describes the configuration of the ZT-40 energy systems and the various programing modes used for the experiments presented in this report.

\section{ENERGY SYSTEMS}

It was initially intended to operate the $2 \mathrm{~T}-40$ experiment with plasma current risetimes of about $10 \mu \mathrm{s}$. However, favorable results from the ETA BETA II experiment at Padua, Italy in the summer of 1979 led to the decision in September 1979 to change the experiment to operate at longer risetimes.

Slow operation was achieved in the following manner. The $10-\mathrm{kV}, 30.6-\mathrm{mF}$ capacitor bank, which was originally intended to suppiy the inftial toroldal magnetic fiela, was disconnected from the $I_{\theta}$ circuit and reconnected to the $I_{\phi}$ circult. This provided a plasma current risetime of $\sim 0.1 \mathrm{~ms}$ with the four feedplates used in the original configuration. With a charge voitage of $8.5 \mathrm{kV}$, plasma currents of $600 \mathrm{kA}$ were achieved.

To provide the toroidal field, the $15-\mathrm{kV}, 2.1-\mathrm{mF}$ capacitor bank, which was originally intended to provide preionization of the $D_{2}$ gas $f 111$, was taken out of a two-stage Marx configuration and connected into the $I_{\theta}$ circuit. It provided toroidal fieids of up to $0.5 \mathrm{~T}$ with a magnetic field risetime of $0.05 \mathrm{~ms}$. Aided reversal was achieved by crowbarring the bank after it had reversed the toroidal field outside the plasma.

Both capacitor banks were crowbarred using the Scyllac-type crowbar systems which were installed to crowbar the $50-\mathrm{kV}$ capacitor banks required for fast operation. The changes in the energy system took less than one week to 
accomplish. Circuit diagrams of the two circuits for this configuration are shown in Fig. II-1, whlch shows the inductance associated with both the start and crowbar systems.

In May 1980, a power crowbar (PCB) was installed in the $I_{\phi}$ circuit. At the same time the crowbar switches were changed to ignitrons in order to handle the Increased amount of coulombs which pass through the switches during PCB operation. Energy from the $10-\mathrm{kV}$ PCB capacitor bank is coupled into the circuit by a 10:1 stepdown transformer. The circuit arrangement is shown in Fig. II-2. The external circuit with ignitron crowbars and PCB transformers is more lossy than the high-voltage passive crowbar circuit. Depending on plasma current, about $2-4 \mathrm{kV}$ charge is required on the PCB bank in order to make up the additional iosses. Therefore, operation of the experiment with low voltage on the $\mathrm{PCB}$ bank is almost equivalert to operation with the high-voltage passive crowbar system.

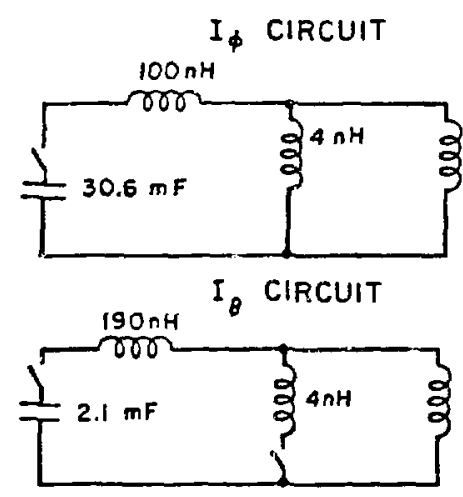

F1g. II-1. ZT-40 circuits.

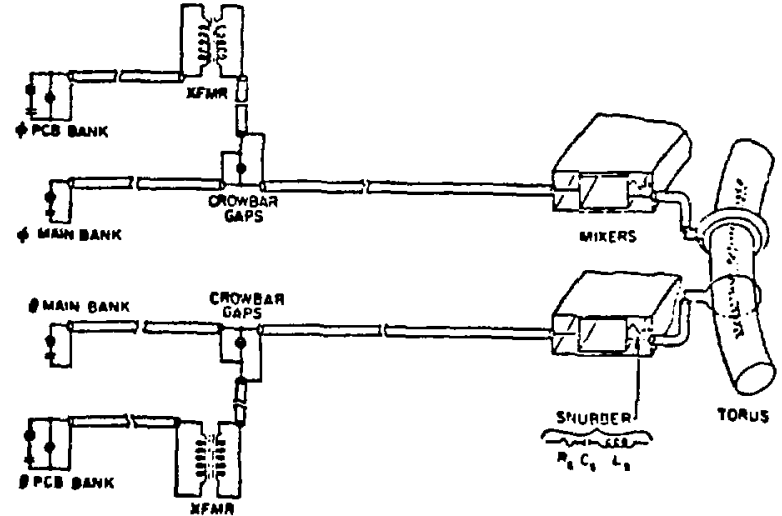

Fig. II-2. Arrangement of $\mathrm{ZT}-40$ energy systems, current mixers, and loads. 
II. PROGRAMMING MODES

The 2T-40 experiment was operated in four different progranming modes. In two of thesse modes, stabilized pinch and self-reversal, the toroidal magnetic field is brought to its peak value and then crowbarred. Simultaneously, the toroldal current is initiated. The difference in the two modes is the size of the toroidal field. In the self-reversal mode the field is small enough so that the plasma current causes a reversal of the magnetic field at the discharge-tube wa1l. Figures II-3 and 4 show the time derendence of the toroldal flux inside the discharge tube, the toroldal field at the wall, and the plasma current for the stabilized pinch and self-reversal modes.

In the other two modes, aided reversal and pitch programming, the toroidal magnetic fleld is crowbarred after the peak field. In the aided-reversal mode, the plasma current is initiated at the peak of the toroidal field. In the pitch-programing mode the plasma current is started

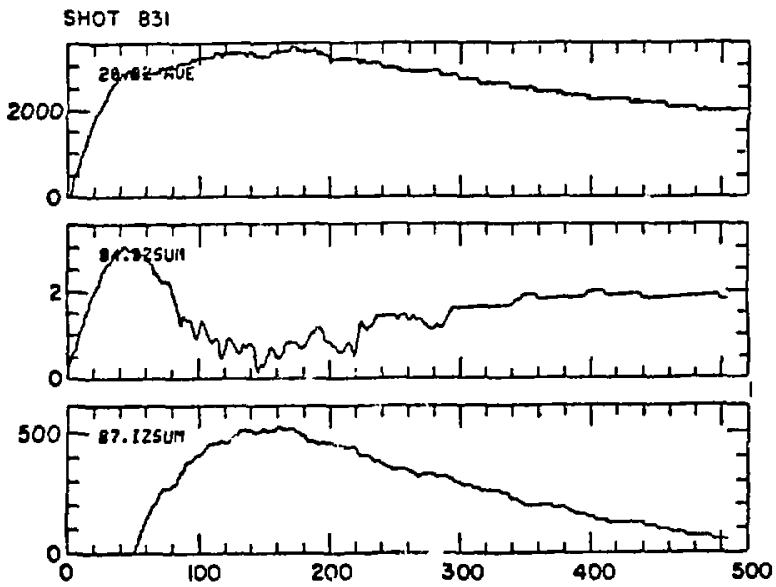

Fig. II-3.

Stabllized pinch operation. $B_{\phi}$ (average) in gauss; $B_{z}$ sum ( $B_{\phi}$ at the wall) in kilogauss; $\mathrm{I}_{\phi}{ }^{\phi}$ sum (toroidal current) in kiloamps.
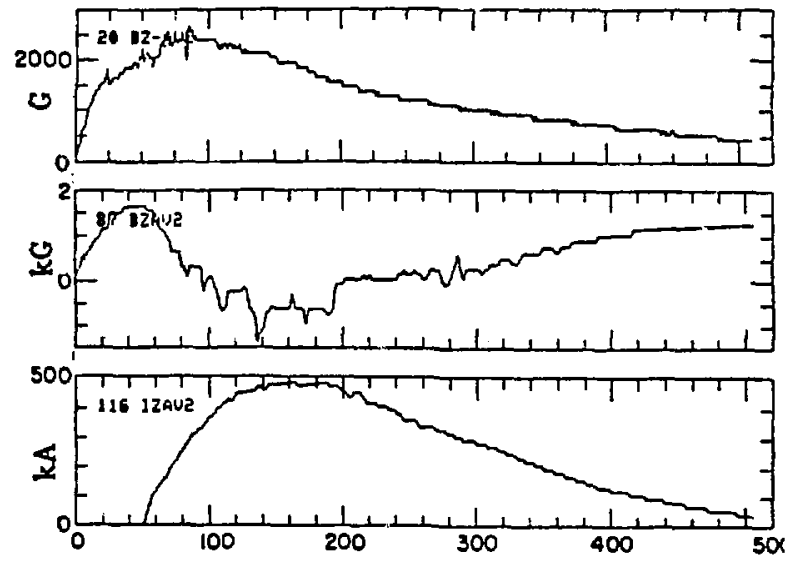

Fig. II-4.

Self-reversal operation. 
$0.02 \mathrm{~ms}$ after the start of the toroidal field in a partial attempt to build in the desired magnetic fleld profiles. The toroldal flux, magnetic field at the wal1, and plasma current for these two modes is shown in Figs. II-5 and 6 .
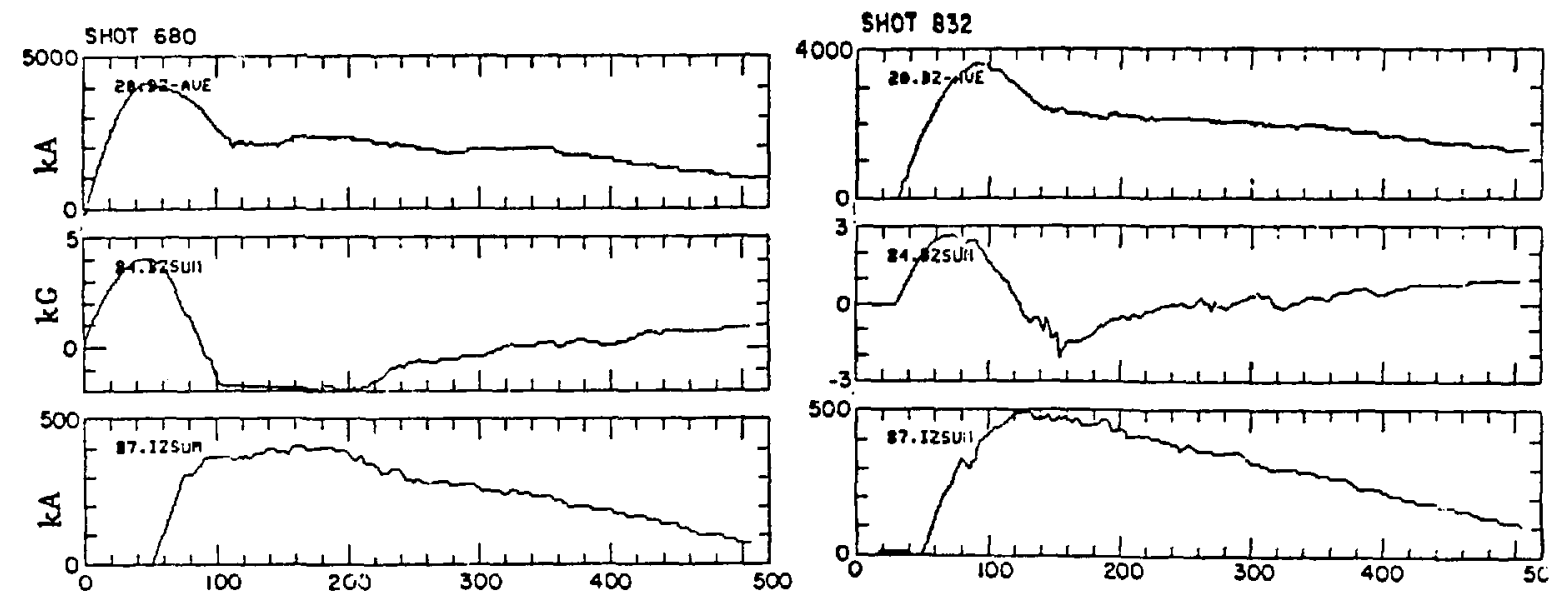

Fig. II-5.

Aided-reversal operation.

Fig. $I I-6$. Alded-reversal operation with pitch programing. 
CHAPTER III

SYNOPSIS OF OPERATING DIAGNOSTICS

by

R. G. Watt

Preliminary to a discussion of operational results on $2 T-40$ in the $100-\mu s$ current risetime mode, let us review what the basic diagnostics available on the machine are. In the northwest section there are two spectroscopic systems, one for the visible region and one in the VUV. Located in the west section there is a two-channel, soft $x$-ray detector system and a 5-chord, fiber-optically coupled visible luminosity system utilizing band pass transmission filters for wavelength discrimination. In the south there is at present a quartz Hilger spectrometer for taking plates in the vfsible. slightly around the torus is a seven-chord interferometer (3.39- $\mathrm{mm} \mathrm{He}-\mathrm{Ne})$. These diagnostics all have extensive data and the results will be discussed in separate sections. In the near future a FIR laraday rotation system will come on-line in the west section. A 2-D Thomson scattering system is to be implemented in the south section. One-dimensional Thomson scattering is already in operation in the north section. The results of that measurement will also be discussed in a separate section.

The preceding diagnostics are direct plasma diagnostics. In addition to these there are numerous electrical dignostics. In both north and west sectors we have toroidal field fluy loops that are constantly monftored. There is a hairpin coll located in the north sector used to measure toroidal field at one point on the minor circunference (outside radius). Also in that sector are two partial Rogowski coils positioned $90^{\circ}$ apart in minor circumference that are used to measure motion of the toroidal current centroid (as well as acting as a pickup for the toroidal current protection circuit, which dumps the $I_{\phi}$ energy if the plasma does not lonize sufficiently quickly, 
to avoid saturating the iron core). In the east sector there is a full Rogowsiki used to measure total current as a backup to a more sophisticated probe system located close to where the interferometer is. This more sophisticated system consists of 24 pickup coils located just outside the ceramic ( 12 for toroldal and 12 for poloidal fields). These loops a:o designed to show plasma motion and distinguish between various types of instability that might exist. At the present time eight of the loops are hooked up to give toroidal current, toroldal field and the differences in these quantities between inner and outer radil. An insertable magnetic probe ( 10 stations radially, $B_{\phi}$ and $B_{\theta}$ ) is 1 the north section but has not yet been inserted on any shots. A simpler, single probe assembly has been used on a limited number of shots with a single feedplate. Finally, there are helical field coils located at four points around the machine designed to pick up particular instability modes. These loops will also be discussed in a separate section. 
CHAPTER IV

BREAKDOWN PHENOMENA

by

R. G. Watt

During the first 1200 shots, ZT-40 had no consistently used prefonization scheme. During this period changes occurred in the current initiation phase of the discharges. When ZT-40 first came into operation it was noted that the unaided toroldal current bank was able to break down the plasma at fill pressures as low as 2 mtorr. Later operations (after 800 shots) revealed that the lower breakdown limit had shifted to $\sim 5$ mtorr. The effect is clearly related to the time necessary to Ionize the gas. This time is related to the hang-up time noted on many similar machines.

Figure IV-1 shows a plot of the hang-up time vs fill pressure for several blocks of shots upon which a pressure scan was performed. Hang-up time as used here is that time necessary to get to $\sim 50 \mathrm{kA}$ after turning on the toroidal current bink. These shots had a charging voltage of 5000-6000 $\mathrm{V}$ on the toroidal current bank. In the earlier shots the toroidal field was slightly self-reversed (toroidal field bank at $4600 \mathrm{~V}$ ), whereas the later shots were stabllized pinch (toroidal field bank at $9000 \mathrm{~V}$ ). The effect of $B_{\phi}$ on the breakdown has been studied elsewhere ${ }^{l}$ and $\operatorname{larger} B_{\phi}$ has been shown to aid the breakdown, the opposite of what is apparent here. This increase in hang-up time over the life of the machine can be explained as a clean-up phenomenon in which fewer impurities were present to aid the sreakdown in the later shots. 


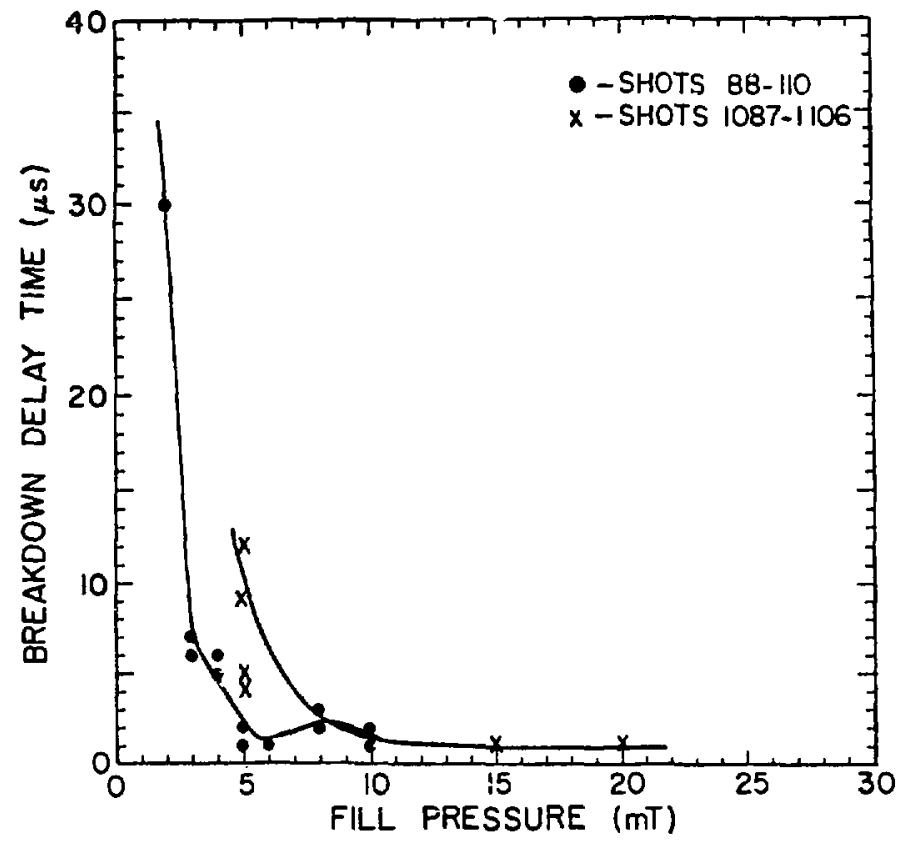

Fig. IV-1.

Breakdown delay time vs fill pressure.

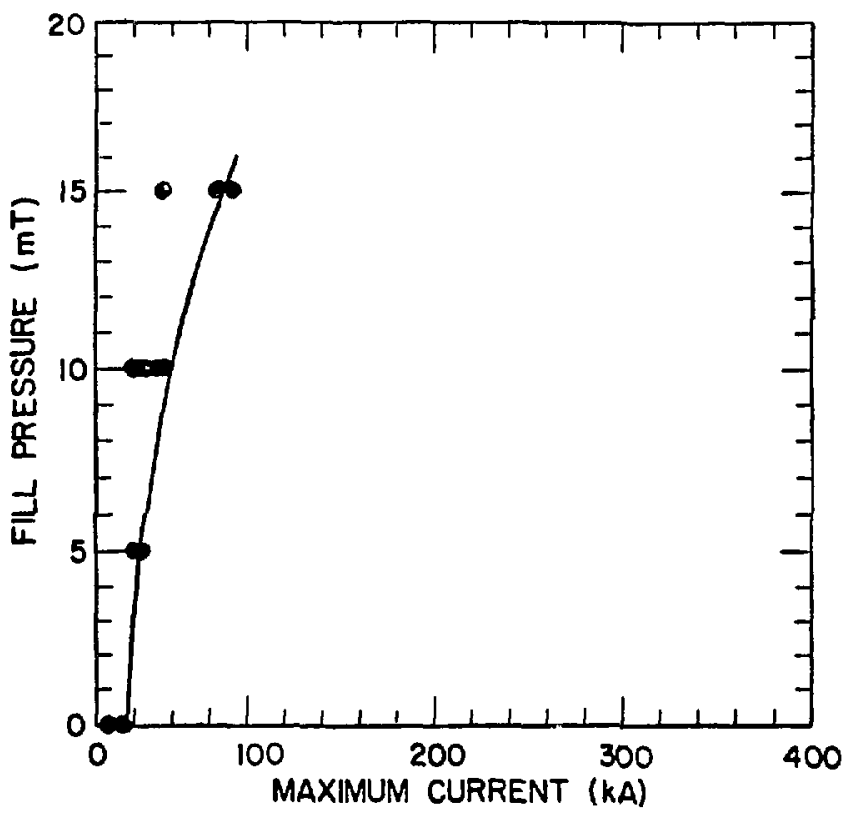

Fig. IV-2.

Fill pressure vs maximum current obtained for a series of shots with constant $v_{z}$. 
Several other pressure-dependent phenomena have been noted in the later shots: a current dependence and decay time dependence on fill pressure. Figures IV-2 and 3 show the peak current and time to decay to $1 / \mathrm{e}$ of peak current as a function of fill pressure for a series of shots in which all other conditions were held constant (stabilized pinch mode). These types of phenomena will be discussed in more detall in the sections on individual modes of operation. This pressure dependence was not obvious in the early shots, but whether it is a manifestation of the clean-up phenomena that probably accounts for the hang-up time is not clear.

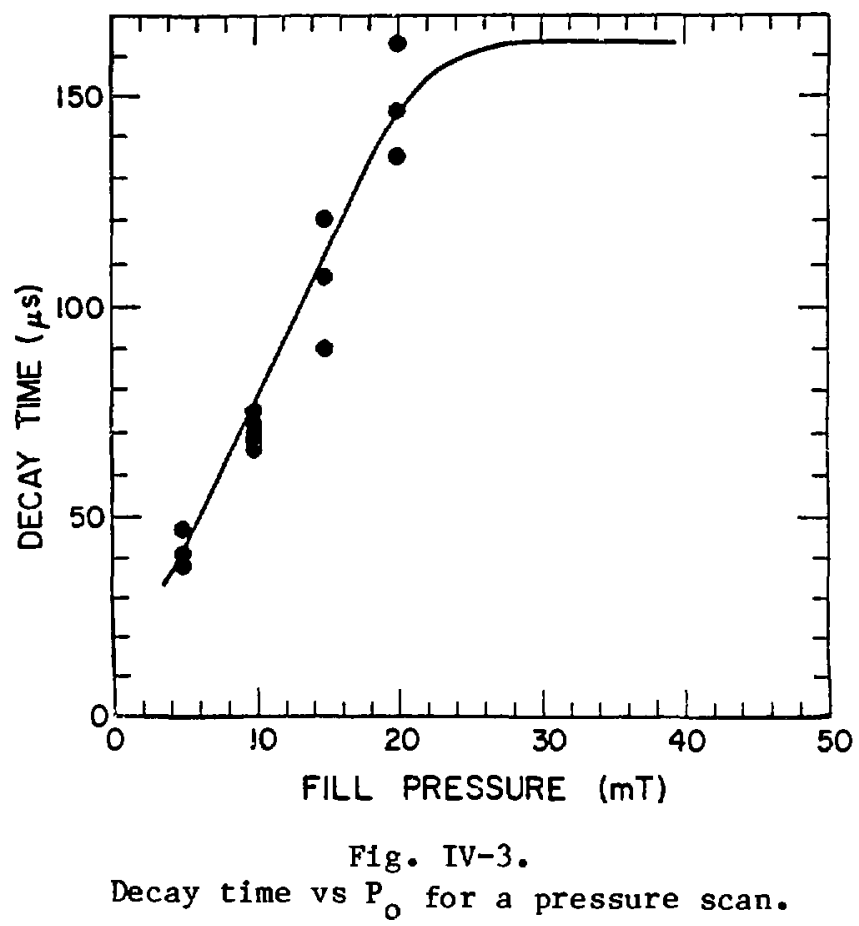

\section{REFERENCE}

1. R. Papoolar, Nucl. Fusion 16, 1 (1976). 
CHAPTER V

RF GLOW DISCHARGE

by

L. C. Burkhardt

An rf glow of a few watts at four stations (see Fig. V-1) was applied to the device with a few microns of deuterfum gas in the torus. The purpose was twofoid: (1) for discharge cleaning, and (2) to aid in the breakdown of the gas before the pre-prefonization current pulse. Figure V-1 shows (a) general

(a)

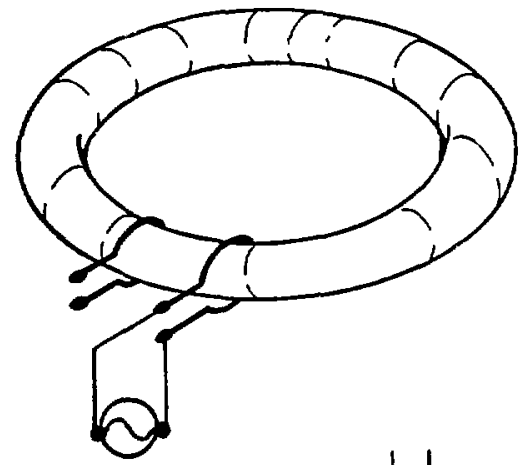

(b)
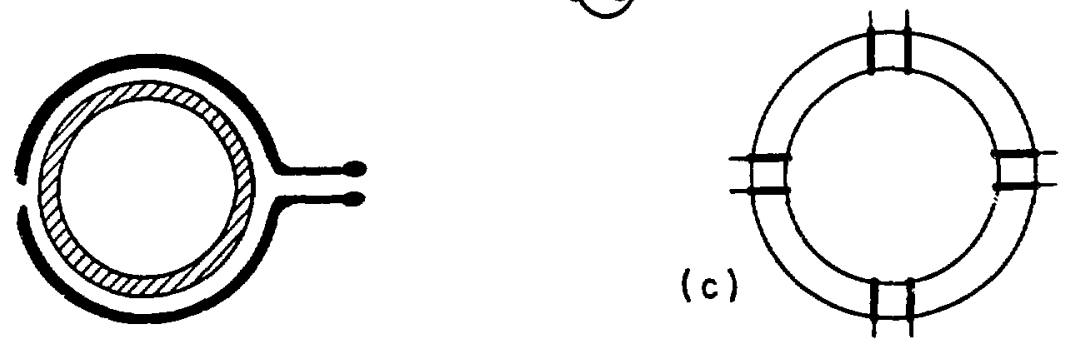

$\mathrm{F} 1 \mathrm{~g} \cdot \mathrm{V}-1$.

Part (a) depicts the general arrangement around the torus, (b) shows the capacitive circuit around the ceramic tube, and (c) shows the coupling locations at four stations around the major circumference of the torus. 
coupling arrangement at each station, (b) capacitive coupling shown as cross section of torus, and (c) all four ZT-40 stations (looking dorm on the machine).

Whenever we exclte the gas for easier breakdown at lower pressures, the effect is the same as has been observed on these devices since the mid-1950s. After the ceramic had torched itself out from several hundred shots, it no longer would break down at $5 \mu \mathrm{m}$, but with rf easily broke down at $2 \mu \mathrm{m}$.

These effects result from a few watts of about $27 \mathrm{mHz}$ rf power being fed capacitively across the diameter of the ceramic at the north, east, south, and west quadrants as in Fix. V-1. Coupling is imperfect, with reflected power about half forward power and approximately $5 \mathrm{~W}$ per station.

The general scheme for rf excitation is laid out in Fig. V-2. More details of the $50-\mathrm{kV}$ isolation transformers are shown in Fig. $\mathrm{V}-3$ at " $\mathrm{A}$ " and "B." At this stage $1 t$ appears $\mathrm{rf}$ does not make any difference at $20 \mu \mathrm{m}$ operation and above. Below $10 \mu \mathrm{m}$ it does, and it is essential below $7 \mu \mathrm{m}$, allowing operation as low as $1 \mu \mathrm{m}$. Also, it appears to be worthwhile, while pumping the machine to achieve a better vacuum, to leave $\mathrm{rf}$ on, such as overnight and during weekends.

The details of this mechanism are not known, but in practice one must start with a glow in the micron range. Then as gas is pumped ous, the glow to the eye becomes fainter and fainter until it is not observable to the naked eye under ordinary seeing conditions. However, rf is stiil effective in the sense that a residual gas analyzer shows different abundances of species with and without $\mathrm{rf}$, even at pressures in the $10^{-7}-\mathrm{mm}-\mathrm{Hg}$ range. Turning the $\mathrm{rf}$

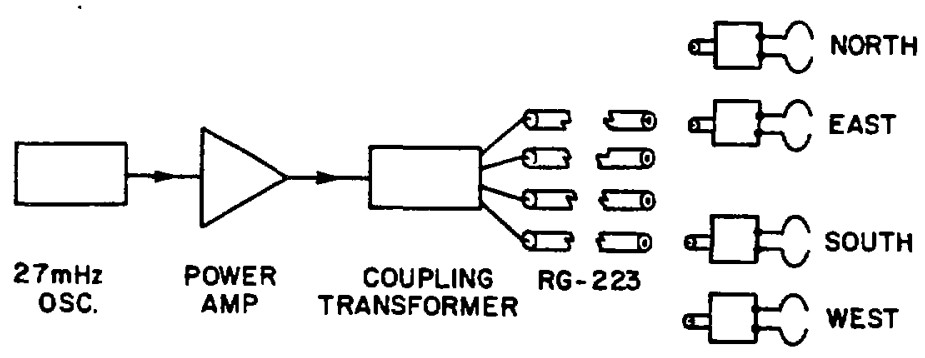
A flow schematic of the $r$ fig. $V-2$.
Fig. V-2. transformer isolating system. 


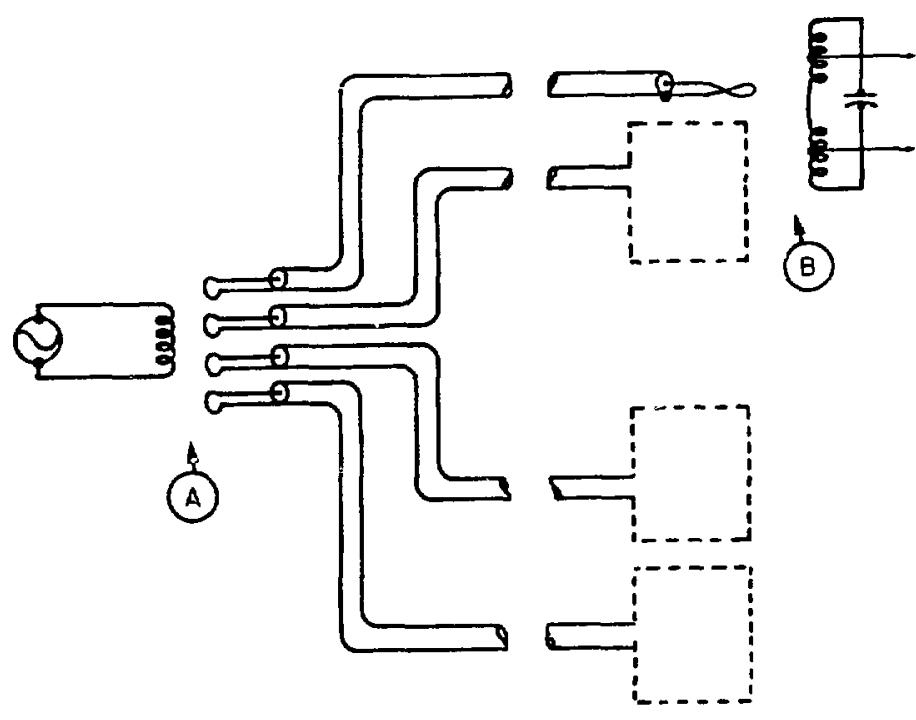

Fig. V-3.

A $27-\mathrm{mHz} \mathrm{cw}$ generator is shown at the left, 1solated at A for $20 \mathrm{kV}$ and also for $20 \mathrm{kV}$ at $\mathrm{B}$; the tuned circuit includes the capacity to the plasma.

power off at low pressures, and then back on does not restore this pumping action. Instead, one must restart. a glow discharge in the micron range and then pump down again wih the rf power on. This effect on the chemistry of residuals (for example, the effect upon the ratio of nitrogen to water vapor) covered in a later section. 
CHAPTER VI

START-UP PHENOMENA DURING FOLR-FEEDPLATE OPERATION OF ZT-40

by

A. R. Jacobson

ZT-40 was operated with four toroidal current feeds uninterruptedly between September 1979 and March 1980. Many modes of operation (stabilized plnch, self-reversal, programmed reversal, etc.) were explored during that period. The results varied widely between operation in different modes. There was a recognizadle trend for the stabllized pinch discharges to deteriorate (for example, become more turbulent, with shorter current decay time) as the fill pressure was reduced below some critical level. This trend was especially surprising in view of the recent experience ${ }^{2}$ of the ETA BETA-II experiment. In that case, low pressure ( $\left.\mathrm{p}_{0}<7 \mathrm{mtnrr}\right)$ was a necessary condition for "good" operation (low fluctuation level, high $T_{e}$, long current decay time), presumably because the oxygen impurity cuuld be "burned through" only at low pressure.

In order to investigate this tendency of the $\mathrm{ZT}-40$ discharge to deteriorate at low pressure, a simple stabilized pinch series of discharges was studied as a function of $f 111$ density, with no other parameters intentionally varied. During the course of two runs (\#880-900 and \$1085-1112) the fill pressure was varied from 55 mtorr down to $5 \mathrm{mtorr}\left(D_{2}\right)$. The toroldal flux was crowbarred (at a density of $2.7 \mathrm{kG}$ ) prior to the current $\Sigma$ ise. The toroidal current rose to $I_{\max } \simeq 350 \mathrm{kA}$, with $\tau_{\text {rise }} \simeq 90$ us. The toroidal fleld (nea: the flux conserver) and current are shown in Fig. VI-1.

As can be seen from the current trace in Fig. VI-1, the decay is almost linear, so a slope is more appropriate than an e-foiding rate. The data for decry slope (as a function of fi:1 pressure) are shown in Fig. VI-?. It is apparent that between 20 mitorr and 15 mtorr a trend begins, in which the decay 
rate becomes greater as the pressure is reduced. (This is the opposite of what one might expect if simple burnthrough of constant impurities were controlling the pinch resistance.)

The sharp transition in decay rate vs pressure between 20 mtorr and 15 mtorr turns out to be well correlated with various measurements made early in the toroldal current rise. In Fig. VI-3 are shown the early feedplate toroldal voltage (one-quarter of "loop voltage") and toroldal current for a 15-mtorr (top) and a 20-mtorr (bottom) discharge. In efther case there is a recognizable "constant Inductance" stage during which both $\mathrm{V}$ and $\mathrm{dI} / \mathrm{dt}$ are constant, beginning at $t \simeq 55 \mu \mathrm{s}$. In the case of $15 \mathrm{mtorr}$, the constant Inductance stage persists for about $15 \mu \mathrm{s}$, whereas at 20 mtorr, 1t lasts for about $20 \mu \mathrm{s}$. The inductance so estimated is shown in Fig. VI-4 as a function of f1ll pressure. The inductance decreases roughly linearly with pressure between 55 mtorr and 20 mtorr. Between 20 mtorr and 15 mtorr the feedplate Inductance decreases more sharply and at st111 lower pressures rises again, in a curve that describes a "big dipper." Because the voltage is measured at the cable header on the feedplate, it is possible to refer the inductance to $r=20 \mathrm{~cm}$ (inside surface of ceramic ilner) by subtracting $0.037 \mu \mathrm{H}$ (the calculated series inductance in the feedplate structure and vacuum gap). The corrected data are summarized in Fig. VI-5. The inductances for $\mathrm{p}_{0} \geqslant 20 \mathrm{mtorr}$ lie roughly on a stralght line with intercept $0.045 \mu \mathrm{H}$ and slope $1.7 \times 10^{-3} \mu \mathrm{H} / \mathrm{mtorr}$.

The measured inductance does not uniquely determine the (diffuse) current profile. However, if one assumes a model, one can make some statements about the influence of $f 111$ pressure. If it is assumed that the current is uniform within an annulus (in minor radius) and zero elsewhere, then the straight-line fit to the data for $p_{0}>20$ mtorr would correspond to an annulus with outer boundary at $r=17.7 \mathrm{~cm}$ and a thickness given by $\delta(\mathrm{cm}) \simeq 0.17 \times p_{0}$ (mtorr). The sharp drop in inductance at $p_{0}=15$ mtorr would indicate an annulus that is thinner and/or further out, compared to an extrapolation of the straight-line fit.

The early-time inductance data, including the dramatic transition at $P_{0}=15$ mtorr, are qualitatively consistent with the interferometer data. In the pressure range $P_{0}>20$ mtorr, the early lonization occurs in a smooth sheath that is peaked toward the outside and that persists until the smooth Implosion occurs, driven by $B_{\theta}$. There is a clear trend in this pressure range 


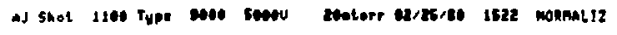

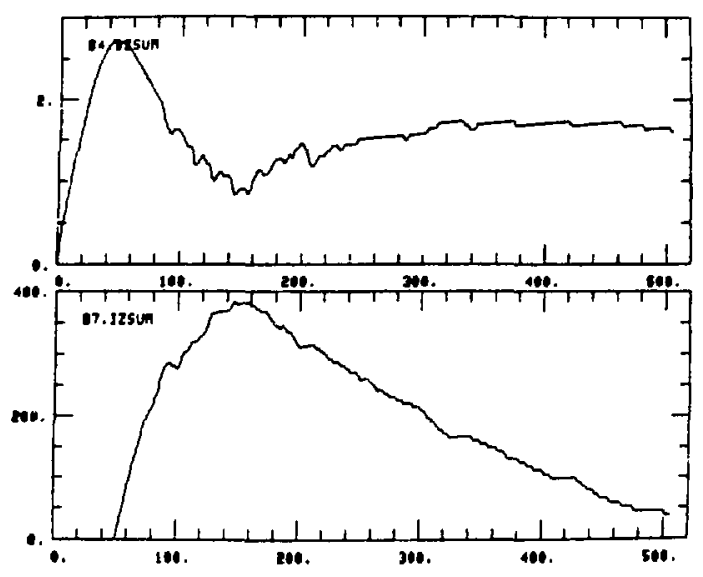

Fig. VI-1.

Top: Toroidal magnetic fleld (kG) vs time ( $\mu s$ ) at conducting wall. Bottom: Toroldal current (kA) vs time ( $\mu s$ ).

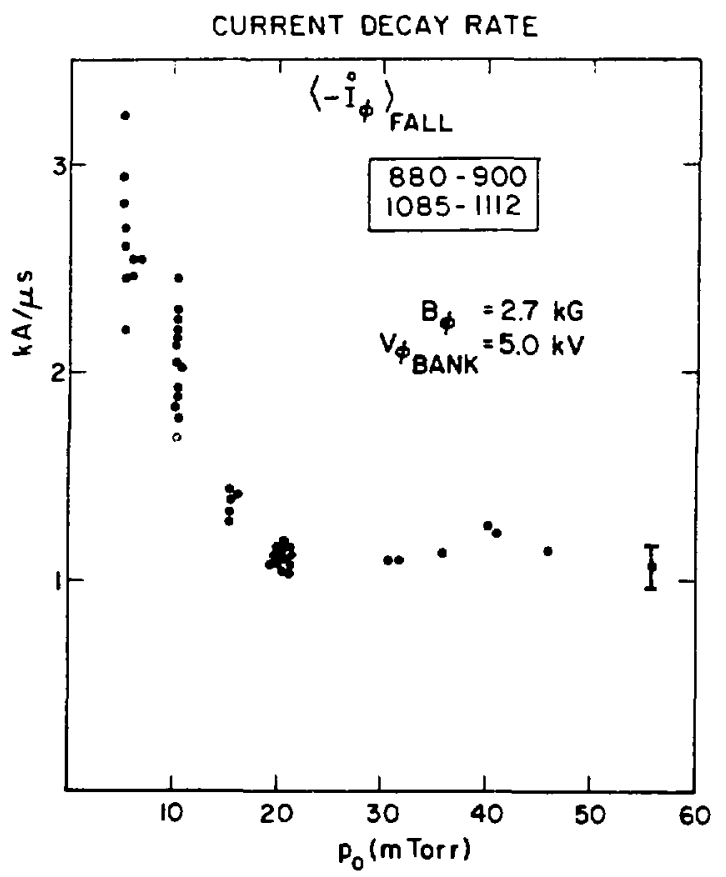

Fig. VI-2.

Toroidal current decay rate during approximately linear fall of current, as a function of deuterium gas $\mathrm{f} 111$ pressure.

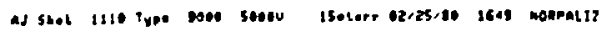

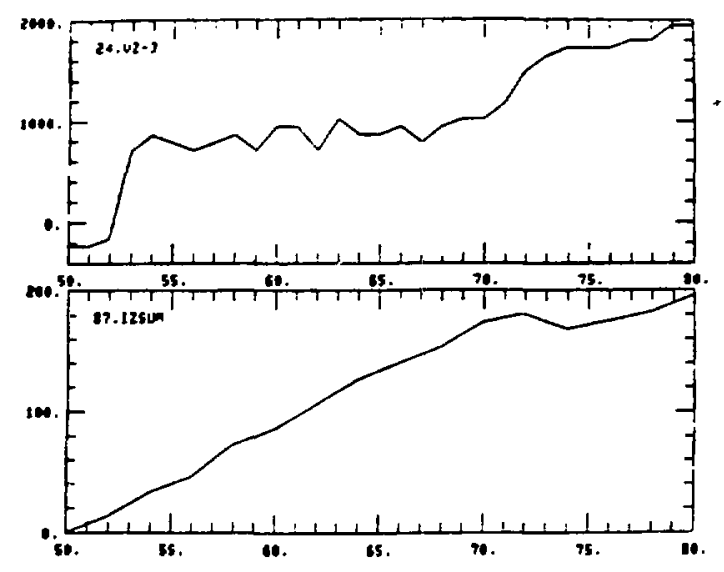

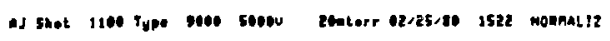

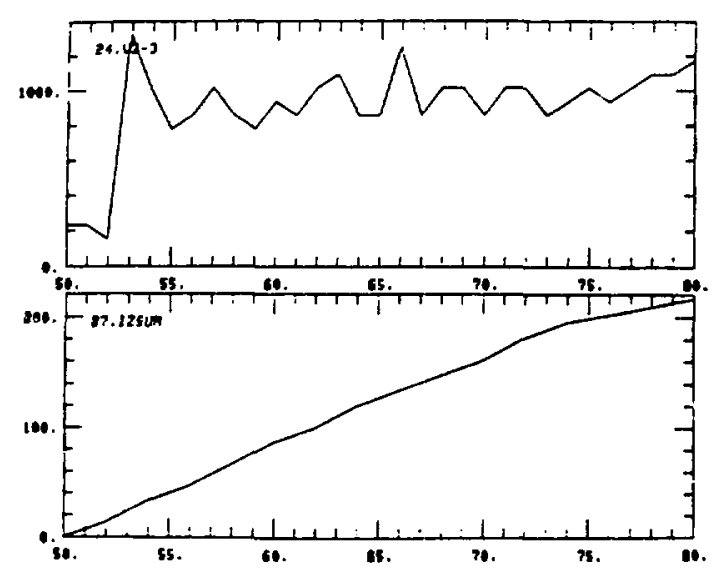

Fig. VI-3.

Feedplate toroldal voltage (volts; one-quarter of loop voltage) and toroidal current ( $\mathrm{kA}$ ) vs time ( $\mu \mathrm{s}$ ) during start-up.

Top: Data for 15 mtorr.

Bottom: Data for 20 mtorr. 
EARLY INDUCTANCE (PER FEEDPLATE)

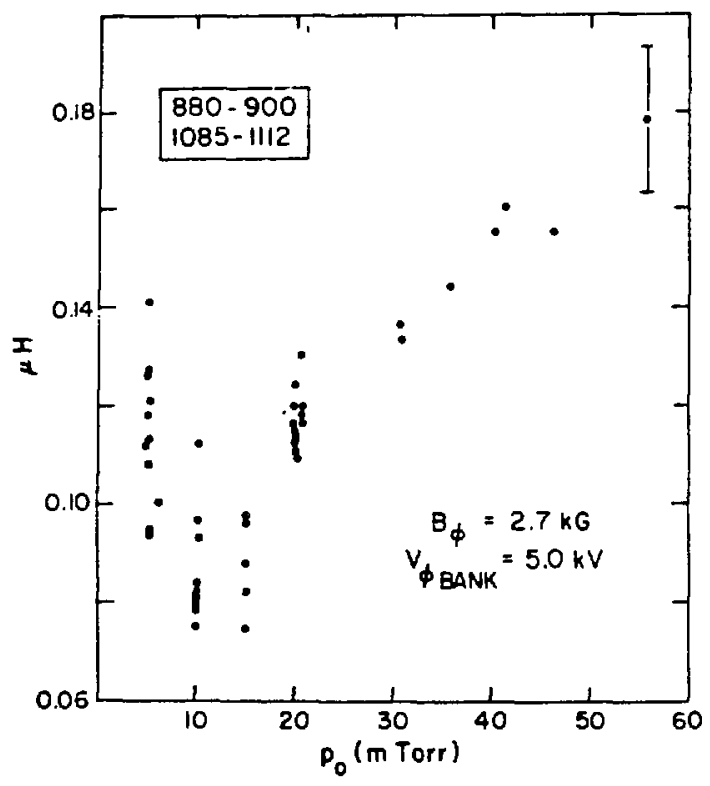

Fig. VI-4

Inductance per feedplate $(\mu \mathrm{H})$ vs fill pressure during early current rise phase of the discharge.

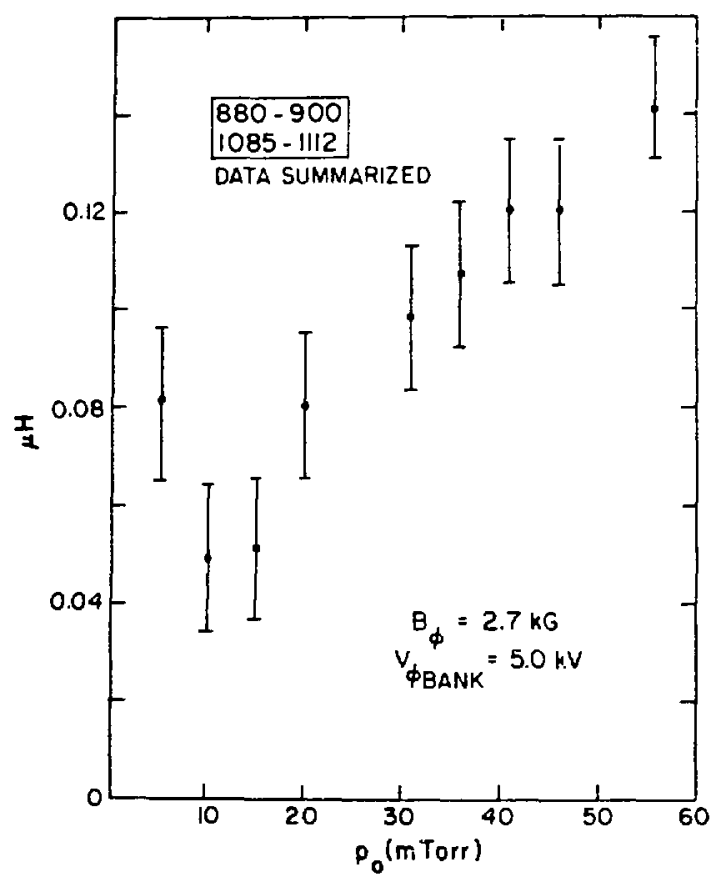

$\mathrm{F} 1_{\mathrm{g}}$. VI-5

Inductance per feedplate $(\mu \mathrm{H})$ referred to $r=20 \mathrm{~cm}$ vs $f 111$ pressure, summarizing data of Fig. VI-4.

for the density sheath to become more diffuse at higher pressure. A qualitative discontinuity occurs at $\mathrm{P}_{0}=15 \mathrm{mtorr}$. Figure VT-h compares the chordal phase-shift data (during the first $30 \mu \mathrm{s}$ of current rise) for $\mathrm{p}_{\mathrm{o}}=15 \mathrm{mtorr}$ and $\mathrm{p}_{\mathrm{o}}=20 \mathrm{mtorr}$. The higher pressure discharge proceeds smoothly, whereas the 15 mtorr sheath becomes disturbed about halfway through the period shown. This transition is quite general: for $P_{0} \leqslant 15$ mtorr the sheath becomes unstable, whereas for $P_{0}>20$ mtorr, the sheath smoothly ionizes and remains intact (during the first 30 is of current rise). It should be noted that $I_{\phi} \simeq 200 \mathrm{kA}$ at $t=80 \mu \mathrm{s}\left(t_{0}+30 \mu \mathrm{s}\right)$.

The same data is presented in Fig. VI-7 in a format more amenable to quantitative analysis. The chordal phase shifts for $p_{0}=20$ mtorr show that after $t \simeq 70 \mu \mathrm{s}$, the innermost chords (located at $R-R_{0}=-5.5,+0.5,+5.0$, and $+7.9 \mathrm{~cm})$ tend to saturate at $\phi \simeq 0.45-0.60$ fringe. The saturation effect in the case of $P_{0}=15$ mtorr is seen to occur 5 us earlier and, more 

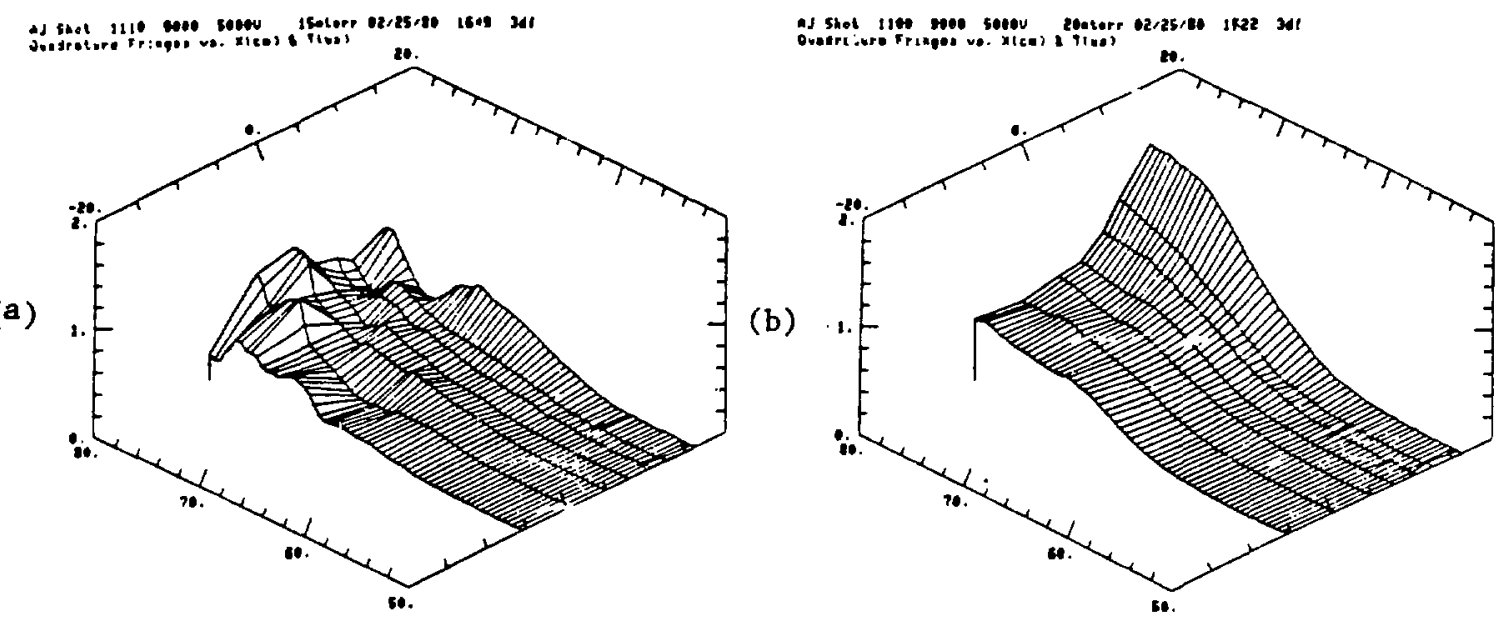

Fig. VI-6.

Interferometer phase shifts (fringes, vertical axis) vs $R-R_{0}$ $(\mathrm{cm}$, from -20 to +20 ) and time ( $\mu$ s from 50 to 80 ) during the first $30 \mu \mathrm{s}$ of current rise. (a) Data for 15 mtorr. (b) Data for 20 mtorr.
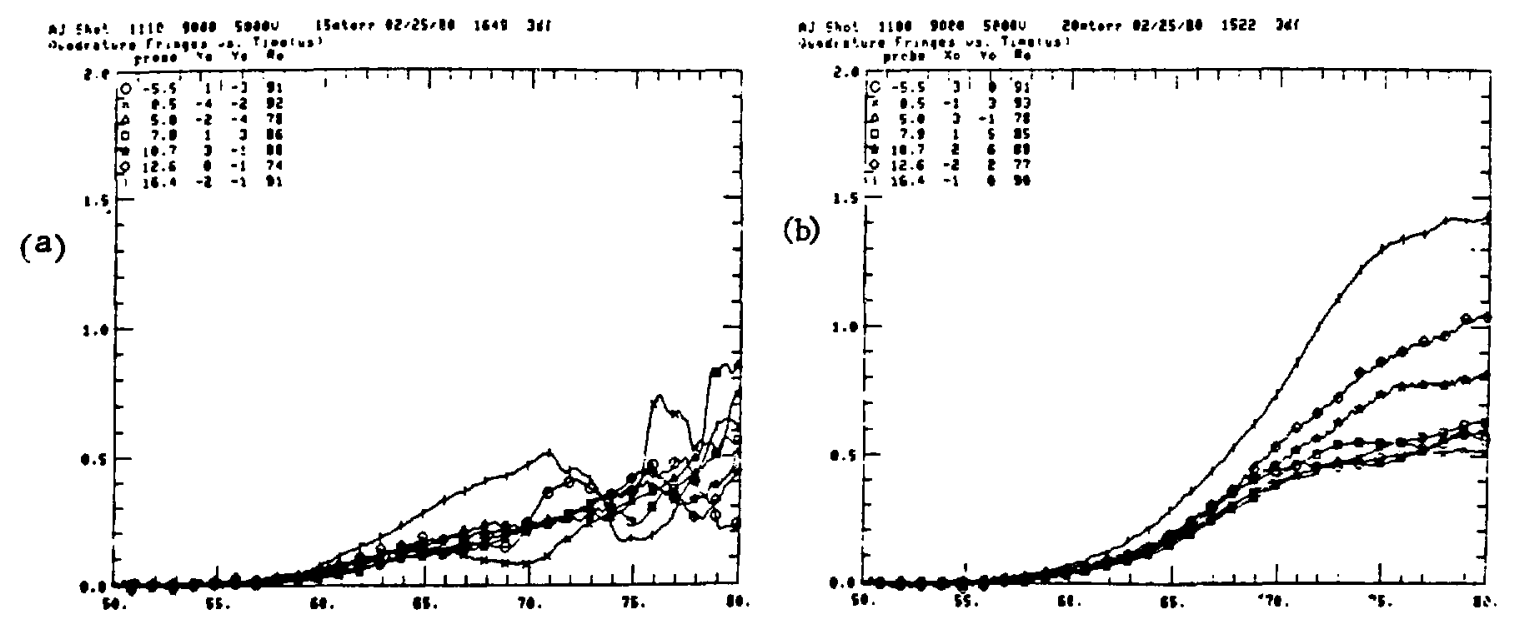

Fig. VI-7.

Same data as Fig. VI-6(a) and (b), but in two-dimensional format: phase shifts (fringes) vs time ( $\mu s)$. The chordal positions (in $R-R_{0}, \mathrm{~cm}$ ) are Indicated by number next to symbol in key, upper left. (a) Data for 15 mtorr. (b) Data for 20 mtorr. 
significantly, at a much lower level, $\phi \simeq 0.10-0.15$ fringe. A1so, density disturbances are seen for $t>70 \mu$ in the low fill pressure case.

The saturation value of the center chords' phase can be used to estimate the effective sheath thickness. In the model, the saturation of the central chords' phase is due to completion of the ionization in the annulus. The tendency of the outer chords' phase to continue to grow might be due either to accretion on the outside or to influx from the wall. Assuming no plasma compression, the saturated central phase shift can be converted to a sheath thickness. This has been done for all discharges ( $\mathrm{p}_{0}>15 \mathrm{mtor}$ ), and the data are summarized at each pressure in Fig. VI-8. Between $p_{0}=55 \mathrm{mtorr}$ and $p_{0}=20$ mtorr, the effective thickness tends to decrease at lower pressures. At $\mathrm{P}_{0}=15$ mtorr there is a sudden large reduction in sheath thickness. The location (in fill pressure) of this discontinuity of sheath thickness corresponds to the inductance discontinulty (see Figs. VI-4 and VI-5). It should be emphasized again that both the inductance and density sheath discontinuities occur at the same pressure ( $p_{0}=15$ mtorr) where the current decay rate starts to rise (see Fig. VI-2).

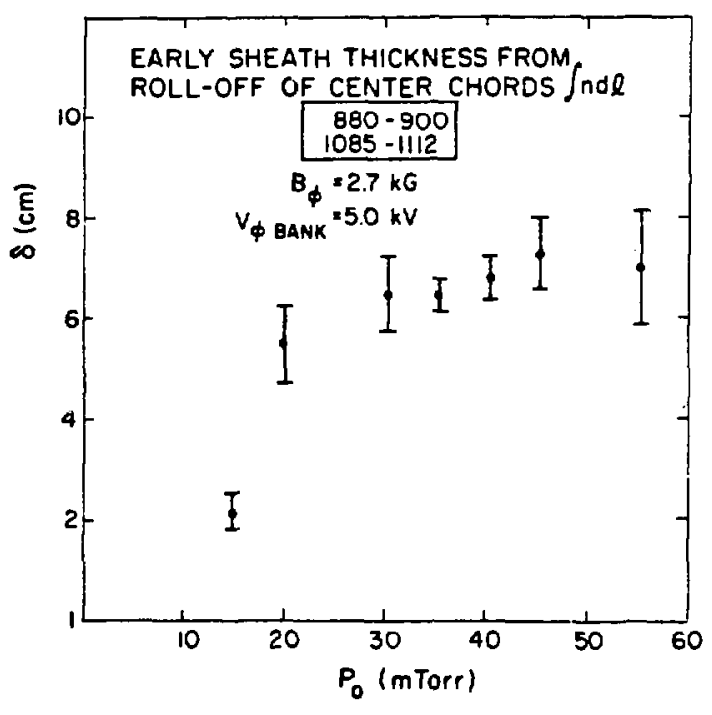

FIg. VI-8

Sheath thickness as a function of fill pressure, estimated from interferometer data early during the current rise. 
The formation of a density sheath that becomes almost fully lonized is supported by data on Balmer $B$ emmission (along a diameter) supplied by R. B. Howe11. The emission signal generally increases at early times, briefly stops after 15-25 $\mathrm{us}$, and then increases much mo $\therefore$. The pause in Balmer $B$ light occurs exactly when the central chords' phase shift saturates. This reinforces the picture of an annulus becoming nearly fully ionized. Figure VI-9 compares Balmer $\beta$ emmission at 15 mtorr and 20 mtorr (for the same shots shown in Fig. VI-7). Comparison of Figs. VI-7 and VI-9 clearly indicates a colncidence in time between saturation of the central chords' phase and saturation of the emission. It is also clear that at 15 mtorr the early resumption of growth in the emmission ( $\simeq 75 \mu \mathrm{s})$ correlates with the onset of density disturbances. Presumably this is due to mixing of the current with the as yet un-ionized gas at smaller radii than the annulus.

Another issue that deserves attention is the formation of runaway electrons at early times. The current channel inductance during lonization (see Fig. VI-5) can be used (along with the $0.037 \mu \mathrm{H}$ "external inductance") to infer the voltage stress per unit pressure at the ceramlc wall. These data are shown in Fig. VI-10. For $p_{0}>10$ mtorr, the variation is weaker than $p_{0}^{-1}$; this is due to the dependence of current channel inductance on pressure in that range of $p_{0}$ (see Figs. VI-4 and VI-5). The recovery of the inductance at

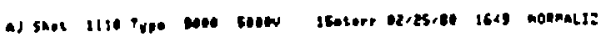

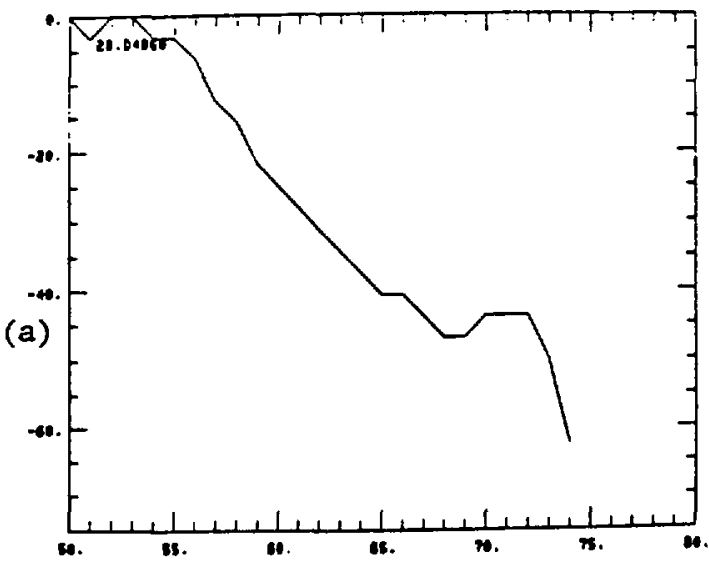

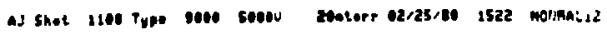

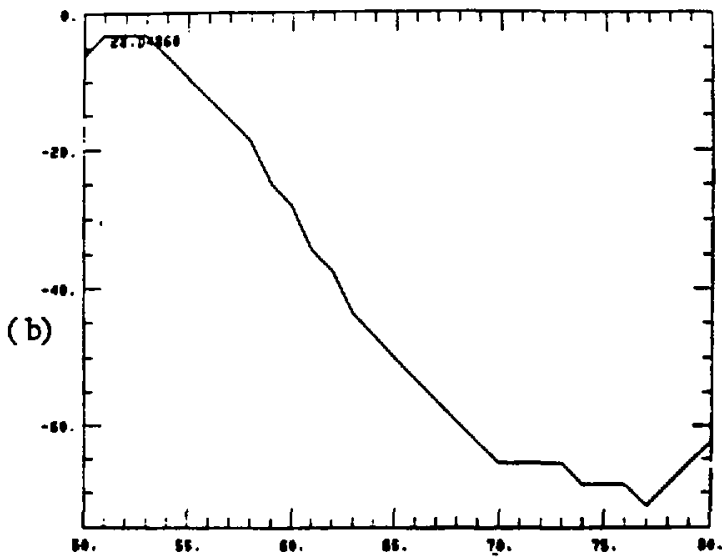

Fig. VI-9.

Balmer beta emission during the first $30 \mu \mathrm{s}$ of current rise (downwards is more emission). (a) Data for 15 mtorr. (b) Data for 20 mtorr. 


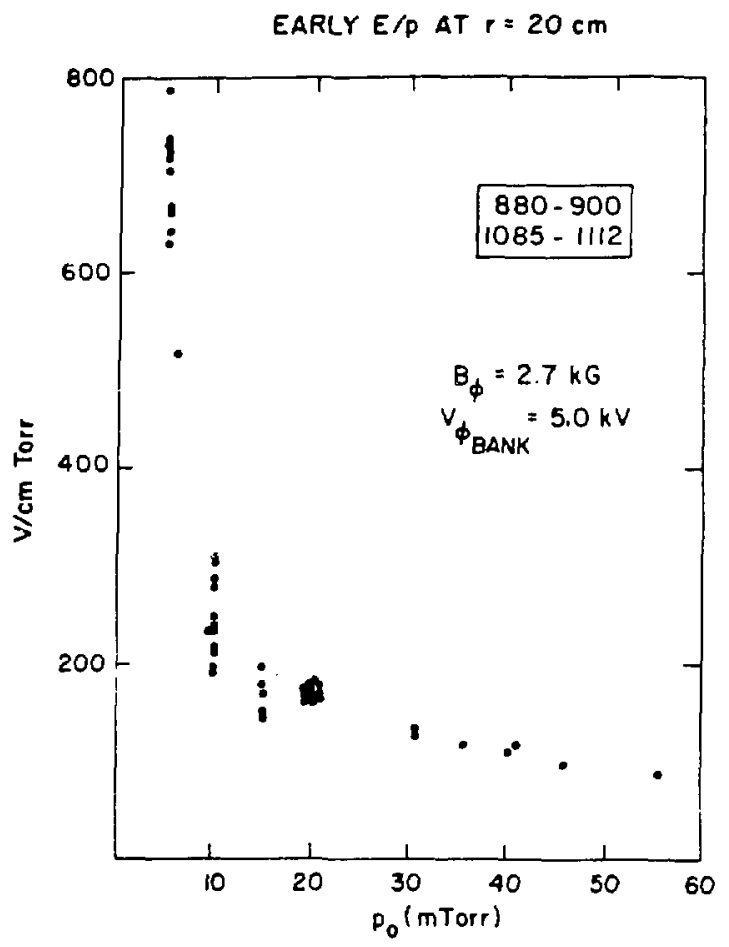

Voltage stress (parallel to toroidal bias field) per unit pressure at the plasma edge during the first $10 \mu \mathrm{s}$ of current rise.

$\mathrm{p}_{\mathrm{o}}=5$ mtorr is responsible for the sudden increase of $\mathrm{E} / \mathrm{p}$ at that pressure (since less voltage is dropped across the $0.037 \mu \mathrm{H}$ external inductance). Runaway formation has been reported in the literature at $\mathrm{E} / \mathrm{p}>200 \mathrm{~V} / \mathrm{cm}$ torr, ${ }^{2}$ so it would not be surprising to find hard $x$ rays at the low end of this pressure scan occurring during the "constant inductance stage," $50 \mu \mathrm{s}<\mathrm{t}<80 \mu \mathrm{s}$. Data supplied by L. C. Burkhardt of photocurrent from $a$ photomultiplier (combined with a sodium lodide scintillator) are shown in Fig. VI-11. (The aluminum primary of $2 T-40$ presumably acts as a shield and provides energy selectivity.) All the 5 mtorr shots have an early runaway signature; none of the other shots do. This is consistent with the E/P data (see Fig. VI-10).

It should be stressed that the deterioration of the pinch at low pressures and the related phenomena at early times are observed in a wide variety of discharge modes. For example, the current decay rate for shots with $40 \%$ more toroidal voltage (and $40 \%$ higher peak current) is shown as a 


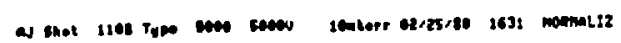

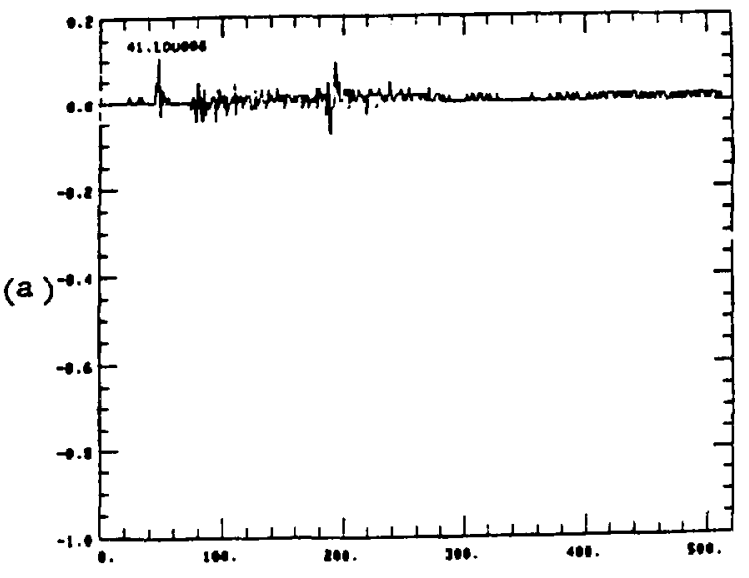

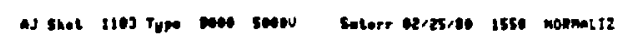

(b)

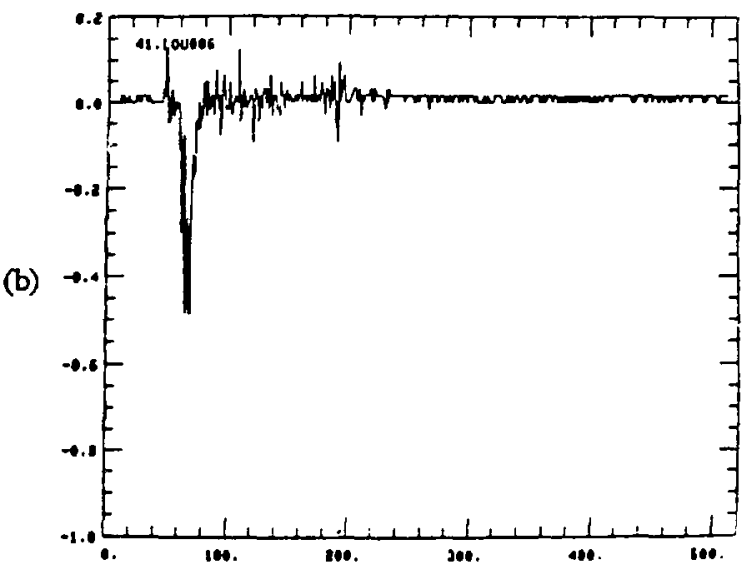

FIg. VI-11.

Hard $x$-ray signal (downwards is more x-rays) vs time ( $\mu s$ ). (a) Data for 10 mtorr. (b) Data for 5 mtorr.

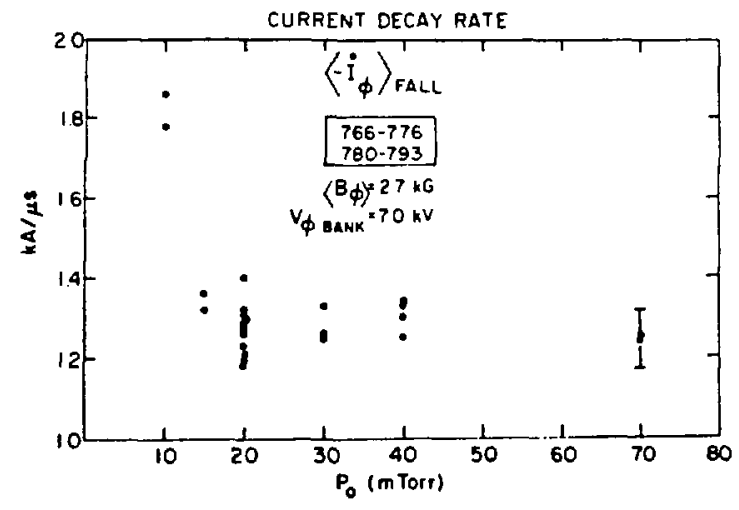

Fig. VI-12.

Current decay rate vs f 111 pressure for a higher-current series of discharges.

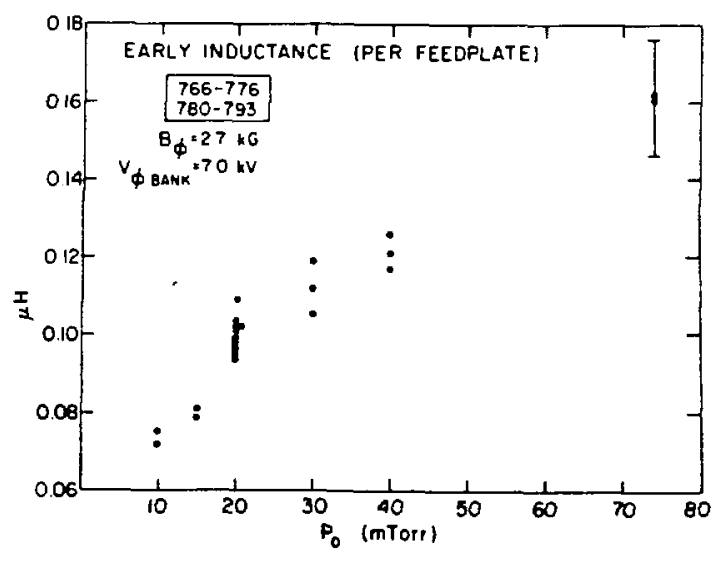

Fig. VI-13.

Early inductance per feedplate $(\mu \mathrm{H})$ vs fill pressure for the higher-current series of discharges.

function of pressure in Fig. VI-12. Again, there is a transition to worse performance for $\mathrm{P}_{0}<20 \mathrm{mtorr}$. The early inductance for that series of shots is shown in Fig. VI-13. Note the discontinuity between $p_{0}=20$ mtorr and $\mathrm{p}_{0}=15$ mtorr. 
The use of weak rf preionization (due to L. C. Burkhardt) does not dramatically improve the discharge behavior (other than facilitating breakdown for $\mathrm{p}_{0}<5$ mtorr). The original pressure scan (\#880-900 and \#1085-1112) was sketchily repeated with a visible glow discharge in the fill gas preceding each shot. The results in terms of current decay rate are compared in Fig. VI-14. The early inductance behavior is shown in Fig. VI-15.

In conclusion, a deterioration of the current decay time is observed at low pressures. A transition is noticeable between $\mathrm{P}_{0}=20$ mtorr and $\mathrm{P}_{\mathrm{O}}=15$ mtorr. At the same place in pressure both the early inductance and early density sheath show dramatic transitions. This correlation suggests that the pinch behavior is significantly controlled by start-up conditions. Hopefully, more diagnosis of the plasma will isolate the memory mechanism (for example, impurities).

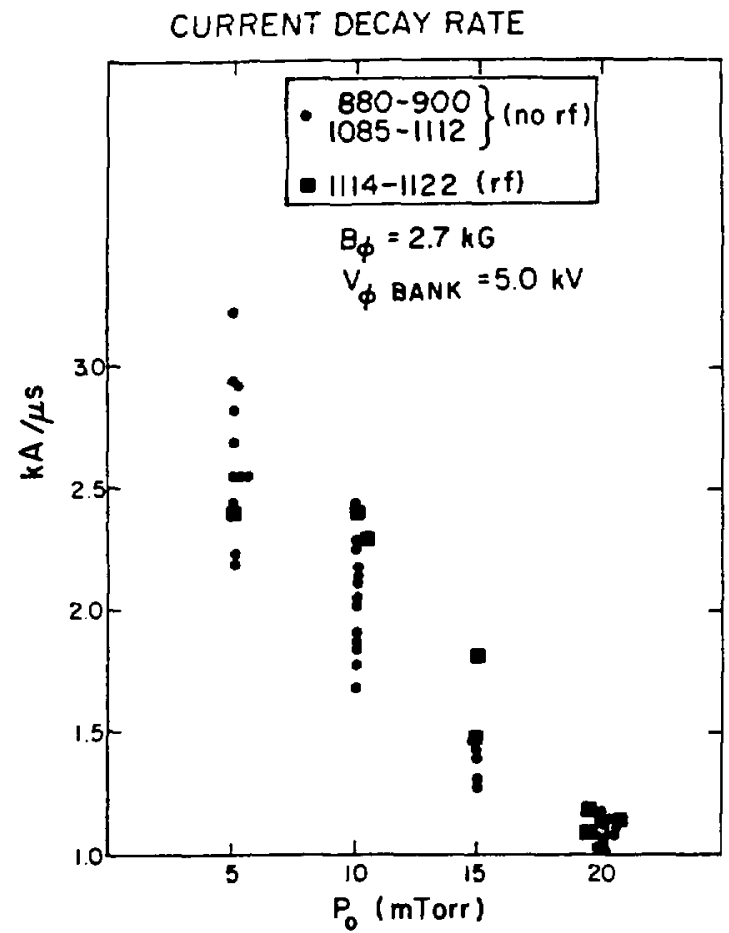

Fig. VI-14. Current decay rate vs fill pressure, with and without rf preionization.
EARLY INDUCTANCE (PER FEEDPLATE)

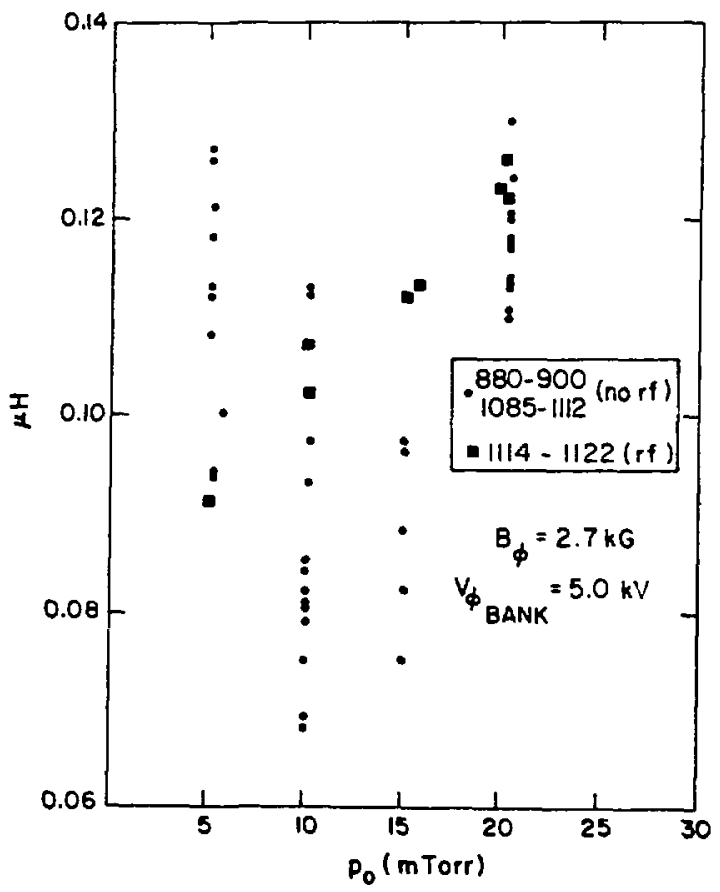

Fig. VI-15. Early inductance per feedplate $(\mu \mathrm{H})$ with and without $\mathrm{rf}$ prelonization. 


\section{REFERENCES}

1. A. Buffa, S. Costa, R. DeAngelis, J. N. DiMarco, L. Giudicotti, G. Malesani, G. F. Nalesso, S. Ortolani, and P. Scarin, "First Results from the Eta Beta II Experiment," Ninth European Conference on Controlled Fusion and Plasma Physics, Oxford, England, September 1979 (to be published).

2. A. V. Gurevich, Sov. Phys.-JETP. 12, No. 5, p. 909 (1961). 
CHAPTER VII

X-RAY AND STREAK OBSERVATIONS

by

L. C. Burkhardt

We Iimit observations to hard $x$ rays; in this case "hard" is defined as emanations capable of penetrating the ceramic discharge tube plus the one-inch aluminum primary shell of ZT-40. Three classes of $x$-ray detectors were used: a dosimeter, thermoluminescent detectors (TLDs), and a sodium-iodidetype scintillator plus photomultiplier (PM) combination. We were primarily interested in personnel safety. In order to reach the PM-scintillator, an additional 0.060 inches of brass had to be penetrated by the $x$ rays. The essential information required from the real-time detector was pulse length; the duration of pulses must be known in order to know whether the TLDs are underestimating the dose. The scintillator was placed one meter below the torus and tangent to the outer shell, or primary circuit conductor.

The dosimeter was read about once per month, as were the TLDs; the machine can be fired about 50 times per working day. At no time was a signal above background detected on efther system. Dosages above 0.03 rem would have been seen, which averages to an estimate of about $10^{-4} \mathrm{rem} / \mathrm{shot}$. (Neutron packets also showed nothing above background; an exposure above 0.58 could have been detected, giving a maximum dose rate of about $10^{-4} \mathrm{rad} / \mathrm{shot}$.) Thus radiation dose rates to personnel tens of meters away are not a concern.

of more interest in terms of plasma behavior are the signals' time histories as recorded by the photomultiplier. Signals appeared over a large range of pressures, electric flelds, and toroldal magnetic fields: pressures were in the 5- to 55-mtorr region, electric flelds were 5 to $20 \mathrm{~V} / \mathrm{cm}$, and toroldal $B$ flelds varied between 500 and 2000 gauss. Figure VII-1 gives an overview of $x$-ray emission over a wide range of parameters. Note that in this 

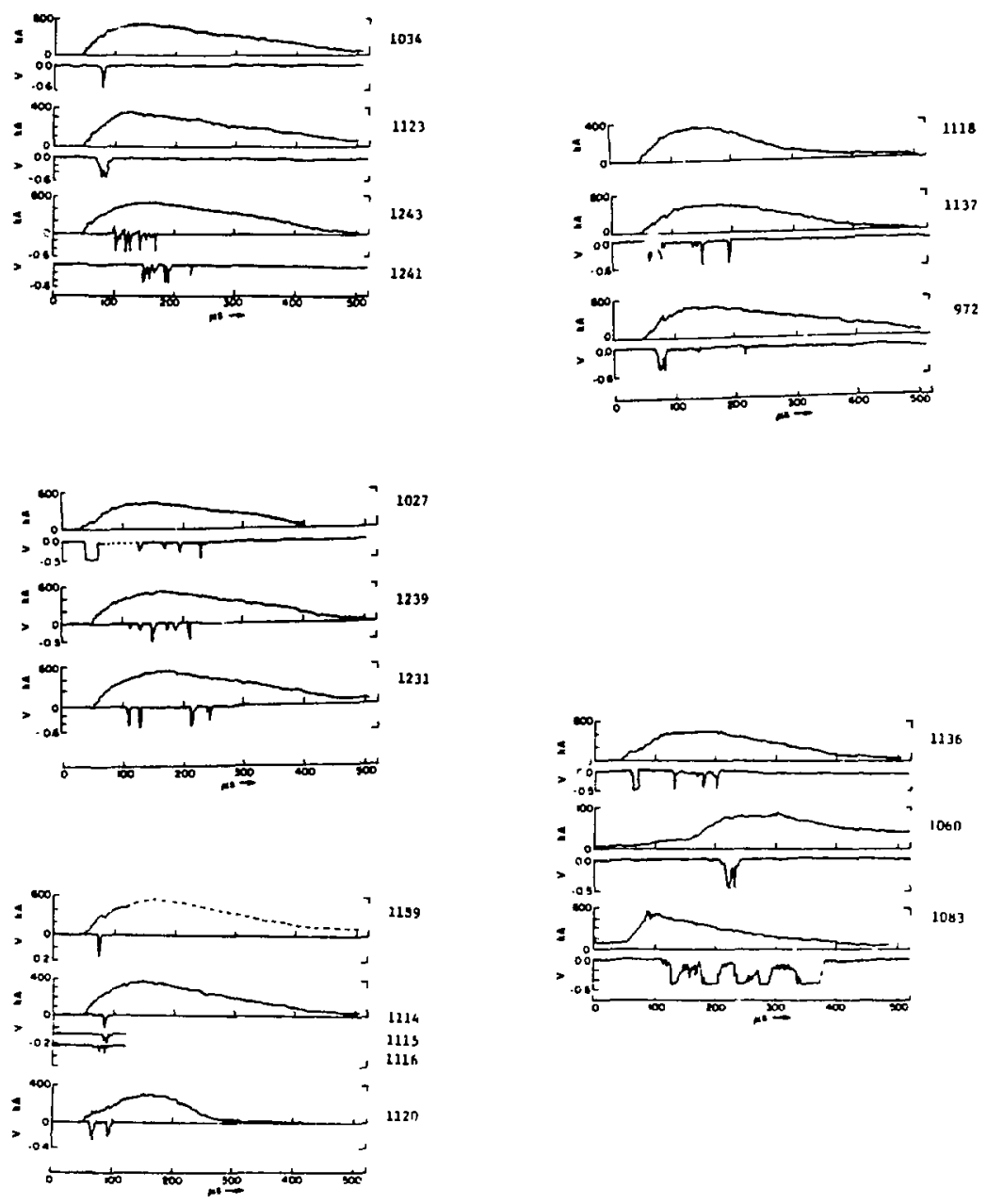

Fig. VII-1.

Overview of $x$-ray production vs time under a variety of plasma parameters.

sampling of events, numbered between 600 and 1245, not all signals appear at the start of toroldal current flow. This suggests the production mechanism is more complex than runaway electrons, or perhaps that there is wore than one production mechanism. 
An attempt was made to correlate $x$-ray signals with changes in the electron line density as measured with Jacobson's seven-chord interferometer (see Chapter VI); A. Haberstich suggested this search. Because it is thought that $x$ rays are produced when runaway electrons hit the walls of the torus, one might postulate that the time of appearance of $x$ rays is synchronous with changes in the electron line densities of the two outer chords of Jacobson's infrared interferometer. Figure VII-2 shows 20-mtorr shots with 3-kG toroidal $B$ field and $17 \mathrm{~V} / \mathrm{cm}$ from the I toroldal bank (for shots around 非190). (If changes were perfectly correlated, all points would fall along the $45^{\circ} 1$ ine.) Toroldal currant starts to flow at about $55 \mu \mathrm{s}$. On the basis of these data we conclude a positive correlation can exist between appearance of $x$ rays and changes in the outer chord signals of the infrared densitometer.

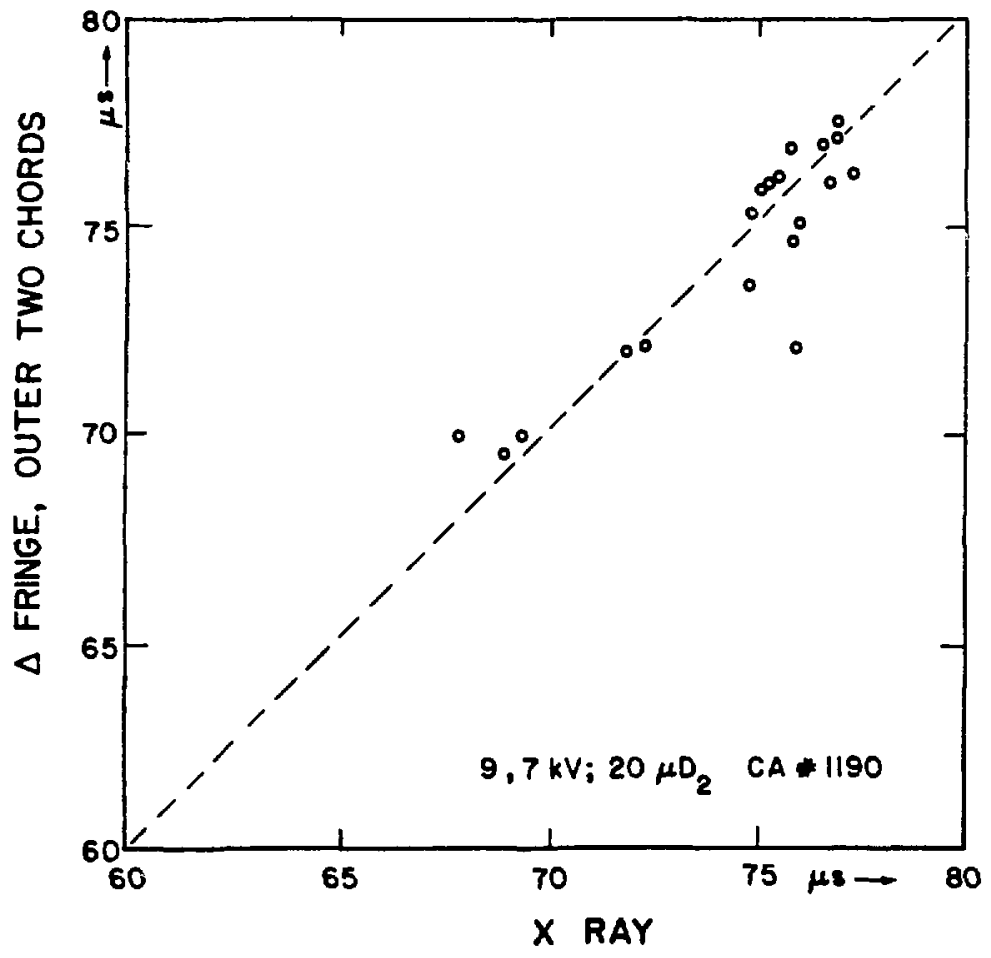

Fig. VII-2.

Fiot showing the positive co:relation between $x$-ray production and changes in electron line densities seen in the infrared densitometer. 
An Imacon 790 camera was used to examine light emanations in the visible through sapphire windows (in the streaking mode). About 800 photos were taken, most of them stereoscopic, looking in the major plane of the torus inwards toward the major axis for one view--and down for the other. The view downward could be correlated with the seven-chord densitometer, because both diagnostics used the same windows in part. Some close correlations indeed were made, provided one looked at changes in luminosity and compared them with changes in ilne density. On fourteen double-blind tests there was one-to-one correspondence. Some work with a yellow and a blue filter $(5714 \pm 61 \AA$ and $4750 \pm 116 \AA$ ) was done as a function of low and high deuterium pressure. Here, as was true in general, streak photography served well as a gross monitor of the operation of the device in various modes of operation (for example, aided reversal incrased the light output). Nonetheless it was difficult to quantify the results. Because the data were not computerized, and because streak photography therefore took more manpower, reliance was eventually put on other diagnostics as they came on-1ine, and particularly because they were able to resolve the spectral emission directly. Most of the streak photos taken were in the shot interval 20 to 1245 , and, as expected in this low pressure, slow-current rise regime did not show the traditional sheath-collapse structure. (The engineering support of Aldred Schofield was important, as was the assistance of Gilhert Maestas and Yashikazu Yoshida.) 
CHAPTER VIII

\title{
AIDED-REVERSAL OPERATION
}

\author{
by
}

R. G. Watt

Among the numerous modes of operation of $\mathrm{ZT}-40$, the one with the longest decay times ( $600 \mu \mathrm{s}$ at $350 \mathrm{kA}$ peak current with $6.0 \mathrm{kV}$ on the toroidal current power crowbar at a f111 pressure of $20 \mathrm{mtorr}$ ) and longest field reversal times ( 225 us for the same conditions) is the alded-reversal mode. This mode is created by allowing the toroldal fleld bank to ring through zero before crowbarring $1 \mathrm{t}$. The result is to let flux escape from the plasma and create a field at the wall that can be as large as ${ }_{\phi}$,peak ${ }^{*}$ The depth of reversal as well as the amount of flux escape is controllable by the time at which the crowbar is applied to the $B_{\phi}$ circuits. A typical set of waveforms is shown in Fig. VIII-1.

The aided-reversal mode produces a lower net current for a given set of bank conditions than any other mode (at 7500 volts on the toroidal current bank, $I_{\max }$ is $\sim 370 \mathrm{kA}$, as opposed to $\sim 490 \mathrm{kA}$ for a stabllized pinch discharge), presumably due to higher compression (due to flux escape) and, consequently, larger plasma Inductance. This effect can be controlled to some extent by the application of pitch programming (that is, changing the time delay between $B_{\phi}$ and $I_{\phi}$ start triggers). By programming (allowing only $20 \mu s$ between the two, as opposed to the standard $50 \mu \mathrm{s})$, it is possible to hold $I_{\max }$ up to about $460 \mathrm{kA}$ for the previous conditions even though a similar flux escape occurs in both cases. Figure VIII-2 shows a typical pltch-programmed discharge.

Alded-reversal operations have several benefits that show up experimentally: longer decay times, various types of quiet periods, and the appearance of a flat spot during the current decay. The decay times are 


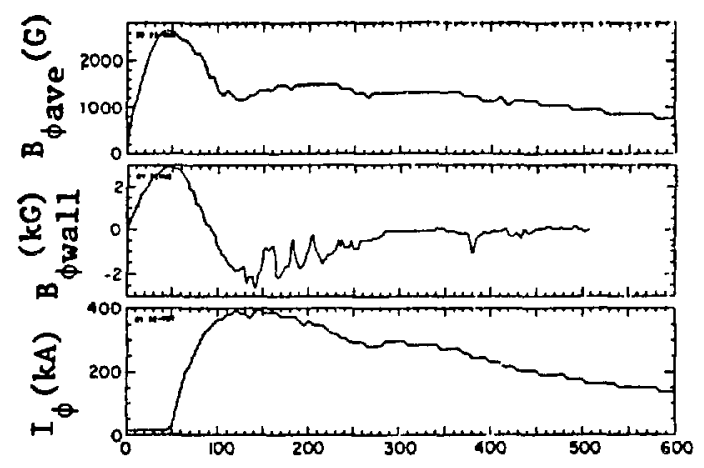

Fig. VIII-I.

Typlcal afded-reversal operation.
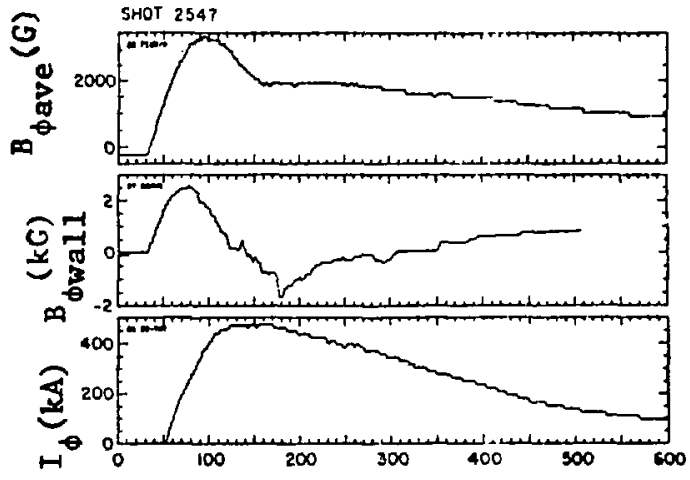

Fig. VIII-2.

Typical pitch-programmed aided reversal for conditions stmilar to Fig. VIII-1.

consistently $30-40$ us longer than the self-reversed case, with identical current levels. Several types of quiet periods occur, one in $\delta n_{e} / n_{e}$ and one in the electrical diagnostics $\dot{\mathrm{I}}_{\phi}$ and the feedplate voltage. The electrical quiet periods, such as Fig. VIII-3, last up to about 100 us and usually collapse (if they collapse) when $\mathrm{B}_{\phi}$ returns above zero. These electrical quiet periods often correlate with a temporary halt in the decay of the current, as shown in Fig. VIII-3. There are also many discharges when the $\dot{\mathrm{I}}_{\phi}$ signal fluctuations never return, as in Fig. VIII-4, but classifying those as quiet periods is not as easy. The electrical quiet periods are usually well correlated with quiet periods in $\delta n_{e} / n_{e}$, as shown in Fig. VIII-5 for the same shot as was shown in Fig. VIII-3. These $\delta n_{e} / n_{e}$ quiet periods usually start earlier than the electrical quiet periods and last longer, but often occur without corresponding electrical quiet periods. Indeed, $\delta n_{e} / n_{e}$ quiet periods were observed long before the electrical ones. What the relationship is, if any, is open to question, but the $\delta n_{e} / n_{e}$ quiet periods seem to be a slightly lower level of discharge fluctuation quietness than the electrical ones. They may represent a marginal discharge from a fluctuation point of view. 


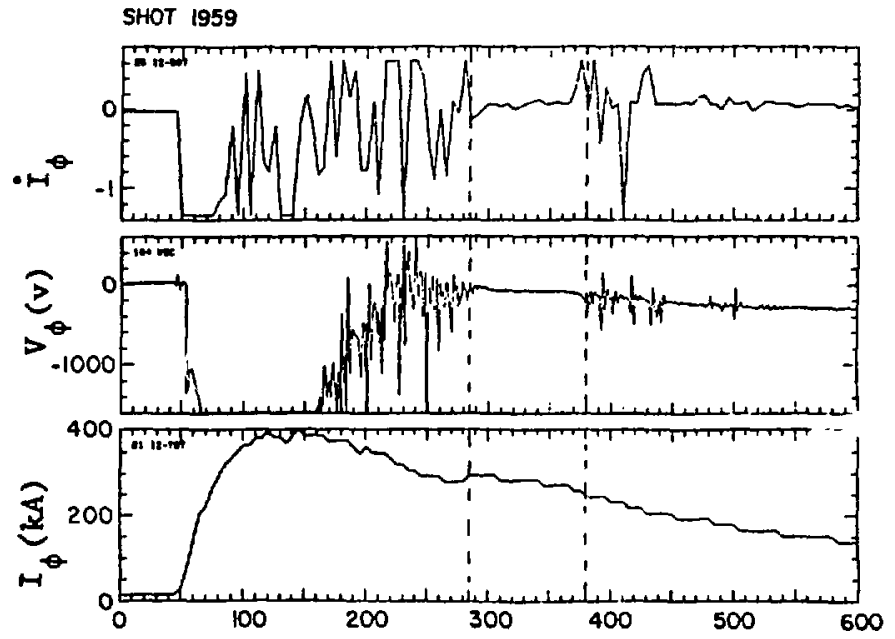

Fig. VIII-3.

Electrical quiet perfods observed in $\dot{I}_{\phi}$ and feedplate voltage diagnostics, and a current waveform from the same discharge showing a flattening out of the decay during the quiet period. The vertical dashed lines denote the interval of reduced fluctuations.

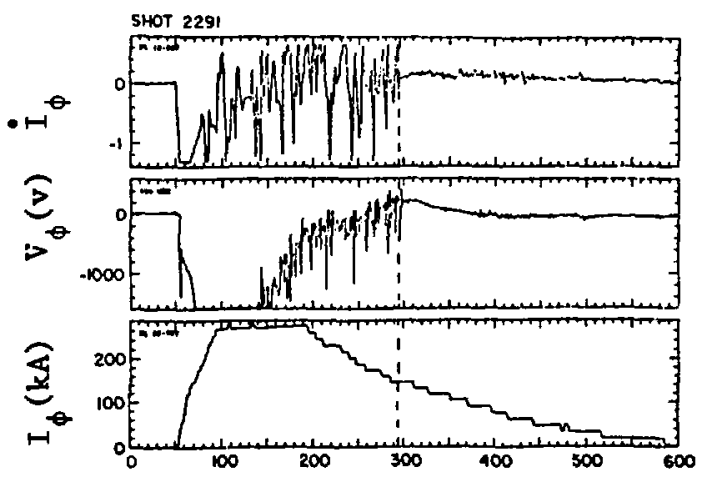

Fig. VIII-4.

The same quantities as in

Fig. VIII-3, but for a discharge in which the fluctuations never returned.

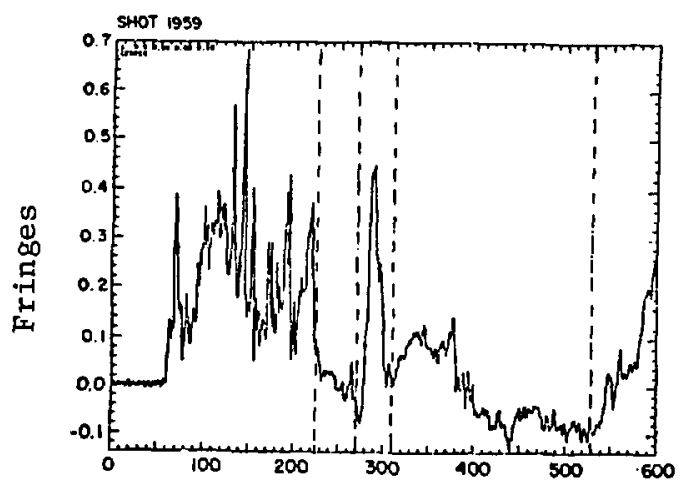

Fig. VIII-5. Interferometer traces showing $\delta n_{e} / n_{e}$ quiet period from the same discharge as Fig. VIII-3. 
Because of severe difficulties with background radiation and low signal levels, it has been very difficult to get any time history of $\mathrm{T}_{e}$ during afded-reversal operation, particularly during quiet periods. What little information is as yet avallable is shown in Figs. VIII-6 and VIII-7, for 3.0 and $6.0 \mathrm{kV}$ on the power crowbar, respectively. As an indicator of a temperature increase or decrease we have a bremstrahlung measurement that may be referred to (see the section on Radially Resolved Luminosity Measurements). Recent information from this diagnostic shows that in the pitch-programmed, aided-reversal mode, during a quiet period, the (density-corrected) radiation levei went down, consistent with an increase in temperature, as shown in Fig. VIII-8. Although this is a very crude indicator, the fact that this is the only such instance of a decrease is encouraging, considering that on $95 \%$ of all stabilized pinch discharges the (corrected) radiation level is rising, consistent with a falling temperature. More work remains to be done in the temperature diagnostic area before any conclusion may be drawn with certainty.

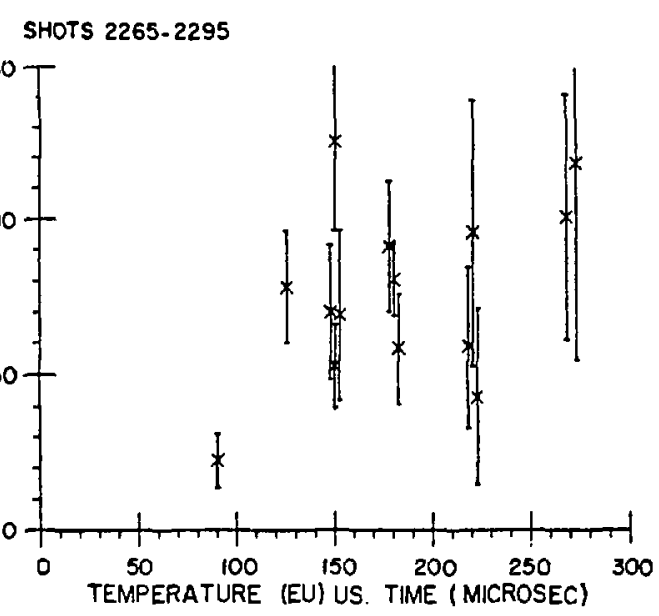

Fig. VIII-6.

$T_{e}$ vs time with $3.0 \mathrm{kV}$ on the power crowbar and a peak current of $280 \mathrm{kA}$.
SHOTS $2311-2332$

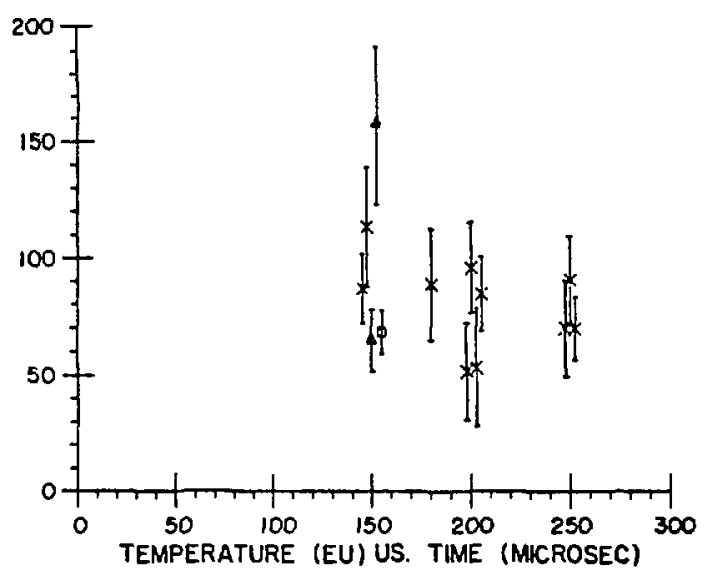

Fig. VIII-7.

$T_{e}$ vs time with $6.0 \mathrm{kV}$ on the power crowbar and a peak current of $280 \mathrm{kA}$. 


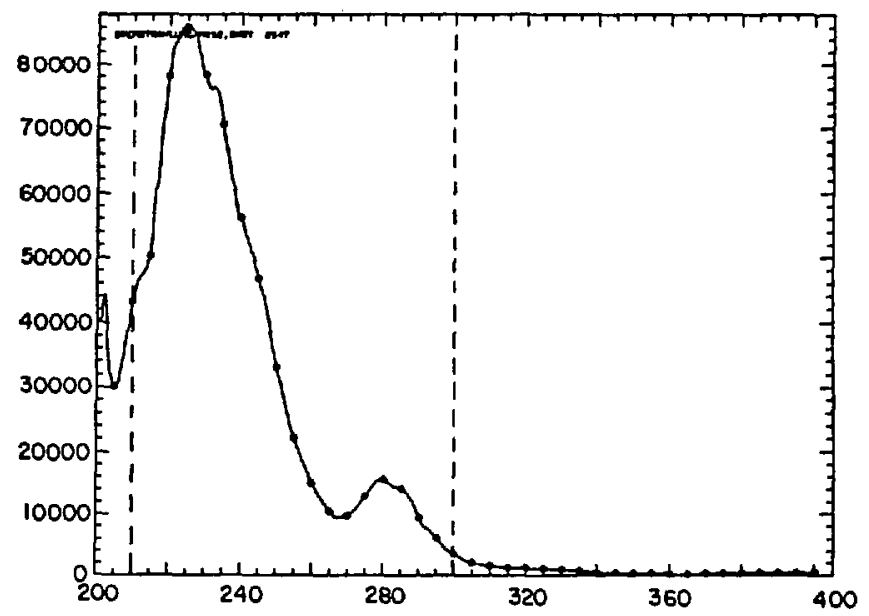

Fig. VIII-8.

Continuum radiation at $520 \mathrm{~nm}$ after correcting for density effects. The radiation should scale like $Z_{\text {eff }}^{2} / T_{e}^{1 / 2}$ so that a decrease could be caused by a temperature increase or a decrease in $Z_{\text {ff }}$ during the shot ( $Z_{\text {eff }}$ Is much more 11kely to increase due to Impurity influx from the wall than to decrease during the discharge). 
CHAPTER IX

SELF-REVERSAL OPERATION

by

D. A. Baker

\section{INTRODUCTION}

Self-reversal is easily accomplished on $2 \mathrm{~T}-40$ owing to the small external toroldal flux reservolr outside the vacuum vessel. The self-reversal operation is accomplished by first applying a toroldal bias field, which is then crowbarred just as the toroldal current is initiated. As the current rises, the toroidal magnetic fleld just inside the conducting shell f.s observed to decrease to a value determined by the ratio of toroidal current to toroidal flux. It is customary to express this ratio as a dimensionless quantity $\theta$, called the pinch parameter, defined as $\theta=B_{\theta w a 11} /\left\langle B_{\phi}\right\rangle$. When discussing the self-reversal process, a second dimensionless parameter, $F=B_{\phi w a l l} /\left\langle B_{\phi}\right\rangle$, is often used to describe the toroidal field at the wall. Self-reversed configurations are characterized by $F<0$. Waveforms for a self-reversing discharge are shown in Fig. IX-1. Shown are the toroldal current, the average toroldal flux (as obtained from a flux loop of radius $21.8 \mathrm{~cm}$ located outside the vacuum wa11), and the external toroidal field outside the vacuum vessel. For this passive crowbar operation, the reversed field normally remains for $\sim 0.1-0.2 \mathrm{~ms}$. It is noted that during the self-reversal process the toroidal flix, which is proportional to $\left\langle B_{\phi}\right\rangle$ in the figure, increases slightly as the positive toroldal flux between the flux measuring loop and the crowbarred toroidal-field colls $a=25 \mathrm{~cm} 1 \mathrm{~s}$ transferred inside the flux measuring loop. The measured electron temperatures for this mode of operation are given in Chapter XI. 

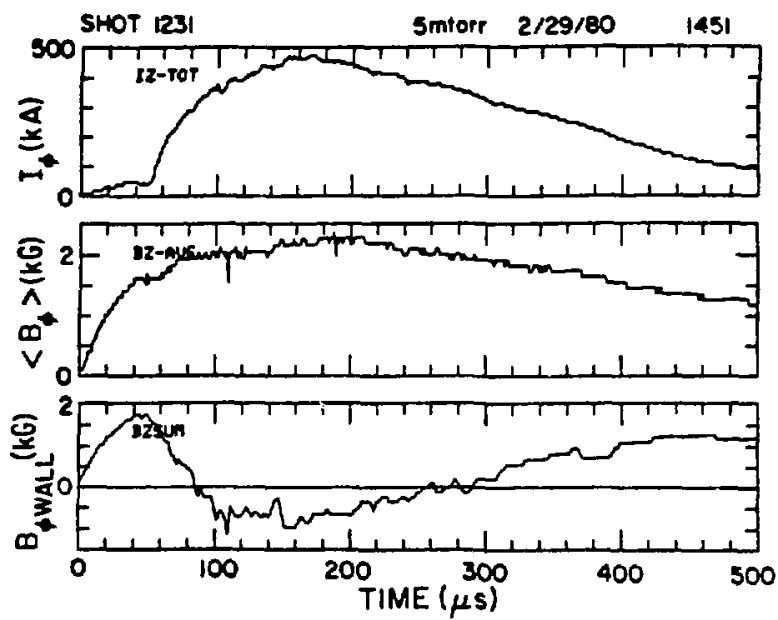

F1g. IX-1.

Toroidal current, toroldal flux, and torofdal magnetic field at the wall for a self-reversed pinch.

II. COMPARISON WITH LOWEST ENERGY STATE PREDICTIONS

The work of Taylor contains the assumptions that the plasma column is totally surrounded by a perfectly conducting wall and that the magnetic helicity $K=\int_{\text {vol }} \vec{A} \cdot \vec{B} d \tau$ is conserved. It predicts that the toroldal field will reverse in the outer portion of the plasma volume for $\theta$ above the critical value of 1.2. The cooling effect of the wall may have an effect in real experiments. It should also be noted that in the RFP experiments the highly conducting shell is not in intimate contact with the plasma and the conditions for Taylor's arguments are not fulfilled. Reversed-field experiments normally have an insulating (as in the case in the present operation of ZT-40) or a resistive liner inside a slotted outer shell. Toroidal-flux conservation applies only to the crowbarred poloidal-field colls on the shell rather than at the conducting shell. Thus there is an ambiguity in the wall radius to use in evaluating $F$ and $\theta$ for comparison with the Taylor prediction. For ZT-40 the critical $\theta$ value for self-reversal is $\sim 1.5$ or $\sim 1.6$, depending on whether the vacuum chamber wall radius or the radius of the metal shell is used. A sample F- $\theta$ diagram evaluated at the vacuum chamber wall is shown in Fig. IX-2. other possible choices of radius for calculating the $F-\theta$ diagram are the radius of the shorted toroidal-field colls or a larger effective radius, which sometimes is used to account for the inductance in the crowbar circuitry. clearly a generalization of the Taylor argument is needed. A difficulty 


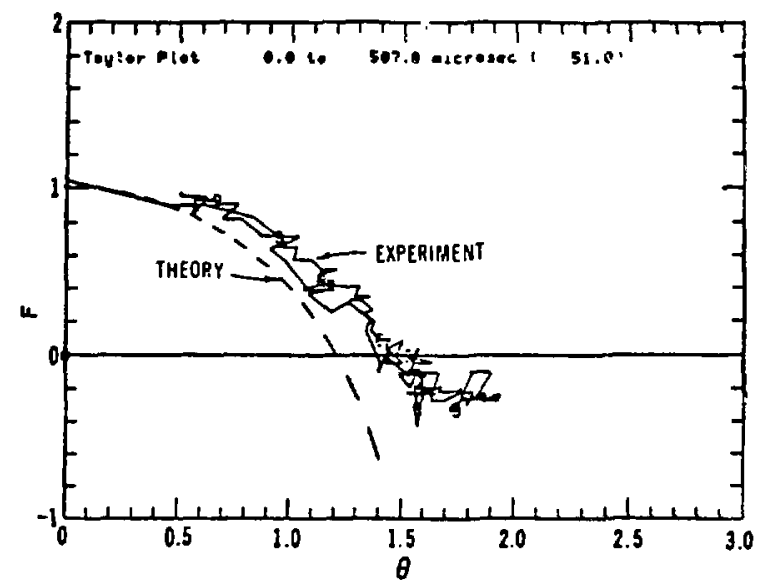

F1g. IX-2.

Experimental Taylor diagram for ZT-40 operated with self-reversal. $F$ and $\theta$ are evaluated at $r=20 \mathrm{~cm}$.

arises in that there is no justification for assuming the conservation of magnetic helicity $K$ inside a volume which includes a region outside the plasma. The observation that the critical $\theta$ value for field reversal occurs for values well above the 1.2 value of Taylor indicates departures from the Taylor states. Such deviations can be obtained by theories which depart from the basic Taylor analysis but still ignore the practical matters just discussed. Such modifications have followed along several lines: (1) incomplete relaxation as a result of resistive diffusion, ${ }^{2}$ (2) the use of different constraints for the deduction of the minimum energy states, 3,4 and (3) the treatment of the transport of the dissipated energy during the relaxation. 5 These approaches deviate from the Taylor result in that they allow states which magnetically contain finite plasma pressure, have low current densities at the wall, and have sufficient freedom to allow field reversal at $\theta$ values near those observed on $\mathrm{ZT}-40$.

\section{DENSITY QUIET PERIODS}

A notable feature of the self-reversal operation was the quiet periods in the density fluctuations as observed on the multi-chord interferometer. A drastic drop in these fluctuations occurred after peak current during the decay and terminated near the time the field reversal was lost. A sample of these phenomena is shown In Fig. IX-3. Such quiet periods occurred in about 


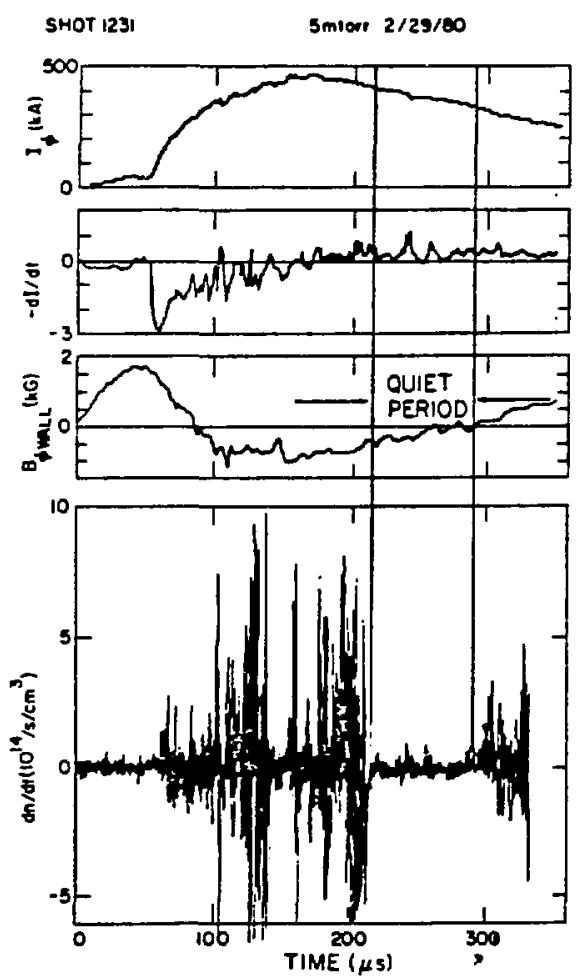

F1g. IX-3.

Quiet period, self-reversed mode. Toroidal current, time derfvative of toroldal current, toroidal magnetic field at the wall, and time derlvative of chord integrated densities vs time; 5 mtorr fill.

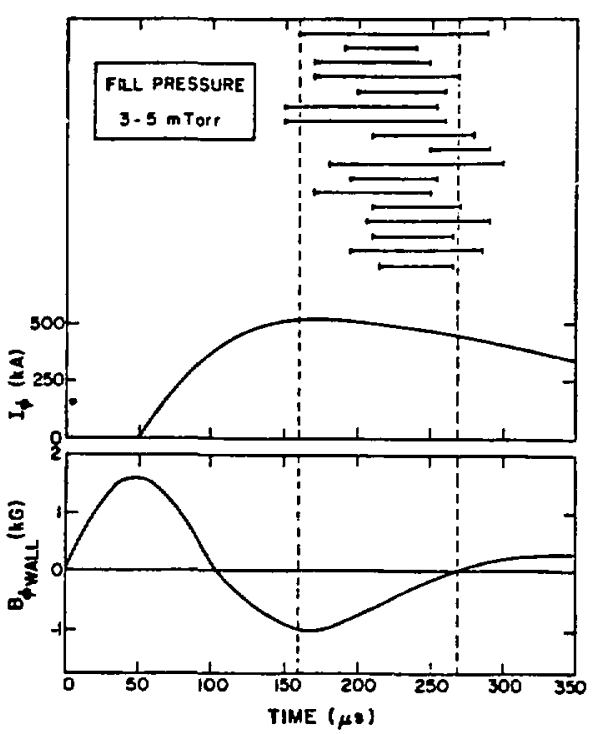

F1g. IX-4. Self-reversed pinch. Toroldal current and field at the wall vs time. Horizontal bars represent duration of quiet density perlods for several discharges.

one out of three discharges and duration of the quiet periods varied, as shown in Fig. IX-4. The vertical dashed lines in the figure show the times of current peak and the time the reversed field is lost, as obtained by averaging over the 17 discharges whose quiet-period duration is shown by the horizontal bars in the figure. These quiet periods differ from those of Zeta, ETA BETA II, and for ZT -40 operated with aided reversal in that a corresponding large drop in fluctuations in the $\dot{I}$ values was not observed. 
IV. CONCLUDING REMARKS

The self-reversal operation is easily obtained and is the simplest method of setting an RFP since no reversed-current programming of the toroldal field circult is needed. One might expect that more turbulence is needed than for an aided-reversal mode in order to obtain the reversed magnetic field and that more wall interaction and impurity release might be involved. The observed temperatures (see Chapter XI) do not appear to bear this out, however. Whether the self-reversal process can be used to maintain the reversed-field state against resistive diffusion and still have acceptable losses has yet to be determined.

\section{REFERENCES}

1. J. B. Taylor, "Relaxation of Toroldal Discharges," Proc. 3rd Topical Conf. Pulsed High Beta Plasmas, Culham, England, September 1975, (Pergamon Press, 1976), pp. 59-67.

2. د. Turner and J. P. Christiansen, "Incomplete Relaxation of Pinch Discharges," Phys. Fluids 24, No. 5, May 1981, pp. 893-898.

j. E. Hameiri, Bul1. Amer. Phys. Soc. 25, 1027 (1980).

4. A. Bhattacharjee, R. L. Dewar, and D. A. Monticello, Phys. Rev. Lett. 45 , 347 (148?!!

5. G. Marklin and A. Bondeson, Bul1. Amer. Phys. Soc. 25, 1027 (1980). 
CHAPTER X

CURRENT DECAY TIMES

by

D. A. Baker

\section{INTRODUCTION}

The decay time of the ZT-40 toroidal plasma current is of interest since it is related to the energy storage and dissipation rate in the plasma. The decay of the toroidal current is controlled by an involved interaction between the plasma and external cirruts which are coupled via the poloidal and toroidal magnetic fields. Studies of the decay time can be conducted using computer codes which use various theoretical descriptions of the plasma physics. The calculations normally involve a complex system of plasma equations, Maxwell equations, and clrcuit equations. The degree of complexity increases drastically as the number of spatial dimensions describing the plasma is increased. The time dependent plasma codes presently avallable at Los Alamos for ZT-40 analysis have, at most, one space dimension and assume the plasma is cylindrically symmetric; they have yet to be coupled to the $\mathrm{ZT}-40$ circuit equations.

II. CURRENT DECAY IN ZT-40

The decay of the toroldal current on ZT-40, when passively crowbarred, is observed to be nearly linear in time (see Fig. X-1). For a nonreversed-field pinch (NRFP) (stabilized pinch), the decay rate increases markedly as the fill pressure is reduced. This effect is correlated with increased density fluctuations as discussed in Chapter VI. The value of the linear current decay rate is plotted vs fill pressure in Fig. X-2 for both NRFP (solid points) and self-reversed pinches (open points). The decays for self-reversed operation are nearly independent of fill pressure. A further observation of 


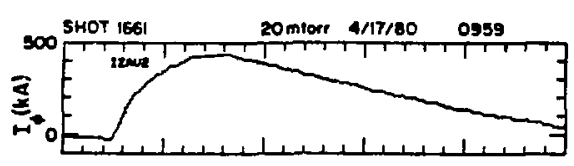

Fig. X-1.

Sample current waveform in ZT-40 showing the linear current decay.

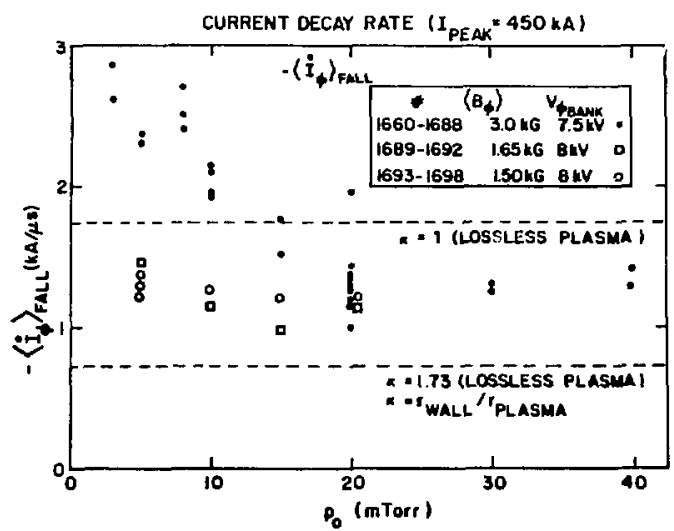

Fig. $\mathrm{X}-2$.

Decay rate of $\mathrm{ZT}-40$ operated as an NRFP (solid points) and a self-reversed RFP (open points). The predicted decay rates for a uniform current density pinch are shown by the dashed lines.

Interest is that a sharp transition in the curreit decay time is seen as the NRFP discharge is converted to an RFP by varying the amount of aided reversal (see Fig. $X-3$ ), which is a function of crowbar time on the field reversing coils. The longest decay time observed in ZT-40 with passive clamping was $\sim 0.40 \mathrm{~ms}$, and as is discussed later, this is maximum value predicted by external circuit dissipation and the estimated plasma inductance.

\section{SIMPLIFIED ANALYSIS}

A simple idealization which can be applied to ZT-40 toroidal current decay time studies is to assume the system can be represented by a series connection of time dependent resistances and inductances representing the plasma and the external crowbarred toroidal current circuit, as shown in Fig. $\mathrm{X}-4(\mathrm{a})$. An alternative approach is to replace the external circuit by the toroldal voltage and current, assumed known from measurements, as shown in $\mathrm{Fig}$. $\mathrm{X}-4(\mathrm{~b})$. The quantities $\mathrm{R}_{e}$ and $\mathrm{L}_{e}$ represent the resistance and inductance of the external circuit after the time of applfcation of a passive crowbar, referred to single turn equivalent values, and $R_{p}$ and $L_{p}$ represent the corresponding plasma parameters. Since ZT-40 has a ferromagnetic coupling of the external circuit to the plasma, the magnetizing current is small and has 


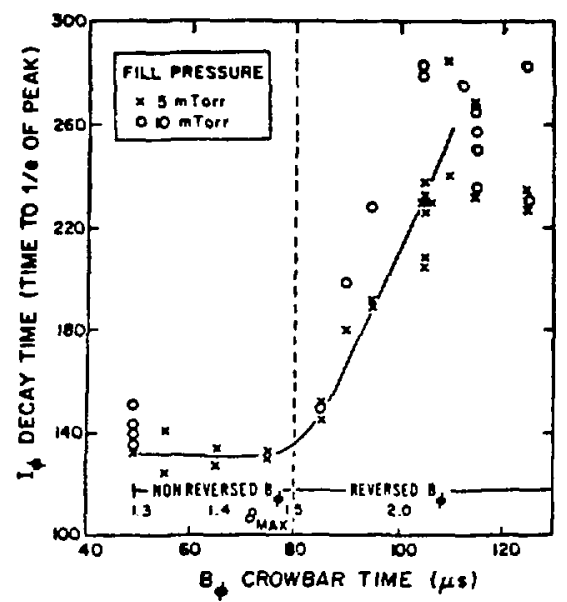

Fig. X-3.

Toroldal current decay time (us) vs toroidal current crowbar time at 5 and 10 mtorr f111. Peak current 400-500 kA.

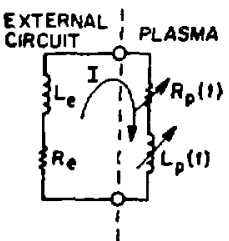

(a)

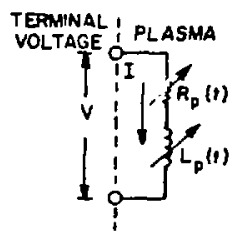

(b)
Fig. $\mathrm{X}-4$.

Simplified $2 T-40$ equivalent circuits.

been neglected. The plasma parameters $R_{p}$ and $L_{p}$ will be time dependent, and since the crowbar clrcuits contain gaseous switches, $R_{e}$ and $I_{e}$ can be time dependent as we11. In addition, field diffusion into the external conductors also gives rise to time-varying external parameters.

Given the observed toroidal current decay wave forms $I(t)$ and $V(t)$, one would like to deduce something about the $7 T-40$ plasma parameters $R_{p}(t)$ and $L_{p}(t)$. We have two prublems: (1) the deduction $R_{p}$ and $L_{p}$ from $I$ and $V$, and (2) the deduction of average plasma parameters from $R_{p}(t)$ and $L_{p}(t)$. We shall address each of these problems in turn.

IV. CIRCUIT EQUATION

We first attack the circuit problem by writing the conventional efrcuit equations for the circuits of Fig. X-4(a) and Fig. X-4(b). For Fig. X-4(a), the equation is

$$
\mathrm{d}(\mathrm{LI}) / \mathrm{dt}+\mathrm{RI}=0 \text {, }
$$

where $L(t)=L_{e}+L_{p}, R(t)=R_{e}+R_{p}$, and $I(t)$ is the toroida1 plasma current. 
For Fig. $\mathrm{X}-4(\mathrm{~b})$, the equation is

$$
d(L I) / d t+R I=V(t),
$$

where $L(t)=L_{p}, R(t)=R_{p}$, with $I(i)$ and $V(t)$ being the toroidal plasma current and the applied toroldal voltage. Since Eq. (1) is a special case of Eq. (2) with $V(t)=0$, a general solution of Eq. (2) will suffice. Whether Eq. (1) or Eq. (2) is used, the basic difficulty in drawing conclusions solely from the current and voltage waveforms is now evident; there is only one circuit equation from which to determine the two unknown functions $L(t)$ and $R(t)$ In terms of $I(t)$ and $V(t)$ (or $I(t), R_{e}$, and $L_{e}$ ). Solving $E q$. (1) for $R(t)$ yields

$$
R(t)=V / I-d L / d t-L(d I / d t) / I
$$

Alternatively, Eq. (1) can be solved for $L(t)$ :

$$
L(t)=\frac{1}{I}\left[L(0) I(0)+\int_{0}^{t}(V-I R) d t\right] .
$$

To make further progress $1 t$ is clear that either more information or assumptions are needed to determine either $R(t)$ or $L(t)$; if one is known, then a knowledge of voltage and current time dependence or external circuit parameters w111 allow the other to be determined.

\section{APPLICATION TO LINEAR ZT-40 CURRENT DECAYS}

As noted earlier, the decay of the ZT-40 current is often very nearly linear in time (see for example Fig. $X-1$ ). It is instructive to apply Eqs. (3) and (4) to the linear decay case for the two special cases when $R(t)$ and $L(t)$ are separately assumed to be constant in time. An experimentally observed linear drop of current can be described as follows. 


$$
\begin{aligned}
& I(t)=I_{0}\left(1-t / t_{d}\right), \quad 0<t<t_{d} \text { and } \\
& I(t)=0, \quad t>t_{d},
\end{aligned}
$$

where $t$ is measured from the beginning of the linear decay and $t_{d}$ is the time required for the current to fall to zero. We will take the approach of Eq. (1) so that $L(t)$ and $R(t)$ represent the sum of the plasmi and external inductances and resistances, respectively. The case of an assumed constant value of $L \equiv L_{O}$, the value of $R(t)$ deduced from $F q .(3)$ (with $V(t) \equiv 0$ ), is shown in $\mathrm{Fig}$. $\mathrm{X}-5$. The resistance varies inversely with the current, becoming infinite as the current approaches zero. The dependence of $L(t)$ for $R \equiv R_{0}$ assumed constant and $\mathrm{L}(0) \equiv \mathrm{L}_{0}$ is given in Fig. $\mathrm{X}-6$. The inductance becomes infinite as $I(t)$ approaches zero with the exception of the case $\mathrm{L}_{0} /\left(\mathrm{R}_{\mathrm{O}} \mathrm{t}_{\mathrm{d}}\right)=1 / 2$, In which case the inductance decays 1 inearly to zero. It is noted that for $L_{o} /\left(R_{0} t_{d}\right)<1 / 2$, negative values of $L(t)$ are required to give a linear current decay. In general, $R(t)$ and $L(t)$ can both vary. It 1 s not possible to determine uniquely time dependences of each unless more data is

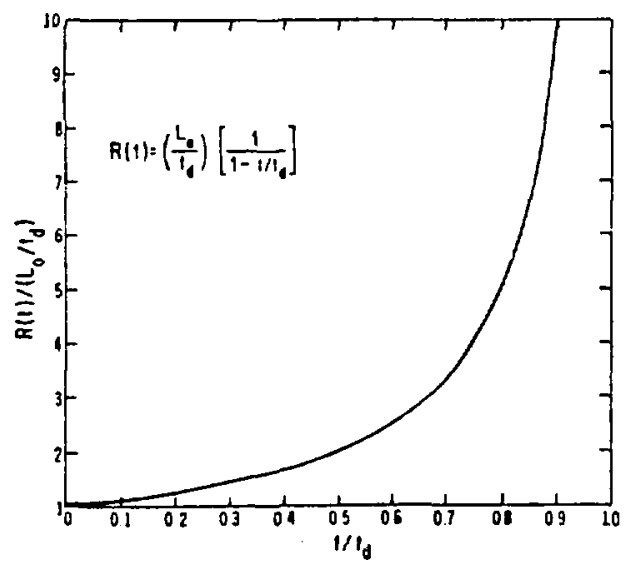

Fig. X-5.

Functional form of resistance needed in circuit Eqs. (1) or (8) (with $\mathrm{V}=0$ ) to cause a linear decay of current from a value $I_{0}$ to zero in a time $t_{d}$ for a constant inductance $\mathrm{L}_{0}$.

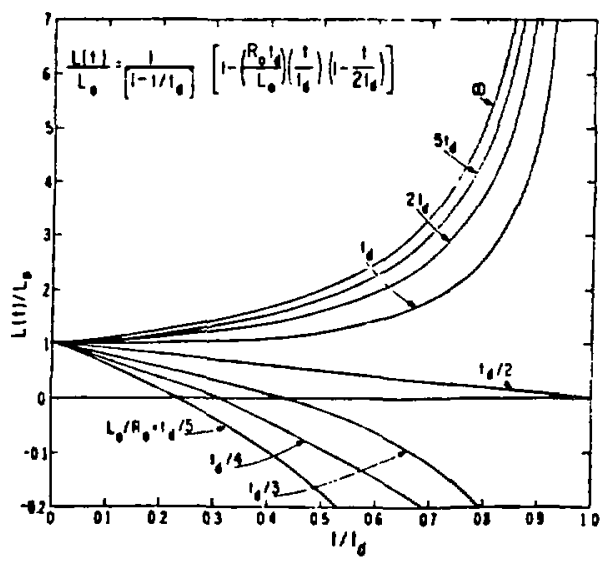

Fig. $X-6$. Inductance needed in Eq. (1) to give a linear decay in current from $I_{o}$ to zero in a time $t_{d}$ if the resistance has a constant value $R_{0}$. $L_{0}$ is the value of $\mathrm{J}$ at $\mathbf{t}=0$. 
avallable than the current and voltage decay waveforms. Internal field measurements would be very useful in this regard.

VI. INTERPRETATION OF THE MEANING OF THE R AND L PARAMETERS

As we have seen, there is a continuum of $R(t)$ and $L(t)$ function pairs which give solutions to Eq. (1) if $I(t), R_{e}$, and $L_{e}$ are known or to Eq. (2) if $I(t)$ and $V(t)$ are known. To be useful, a relationship of $R_{p}$ and $L_{p}$ to the global plasma parameters is needed. For example, we can require that $R$ and $L$ be defined so that $I^{2} \mathrm{~K}$ is the total ohmic dissipation and $\frac{1}{2} \mathrm{LI}^{2}$ is the total stored field energy in the cfrcuit. These interpretations come from standard circuit theory, which are normally derived using thin conductors for which the current can be treated as unfform over the conductor cross section, and the field energy and flux inside the conductors are negligible. For the diffuse plasma column of ZT-40, these approximations are not valid and care must be taken if valid estimates are to be made from circuit equations in lieu of multidimensional computer codes.

A. Stationary Case

For the case of a stationary plasma with toroldal current only, we have energy balance in the plasma column (that is, power in = ohmic dissipation + rate of change of magnetic field energy). For the requirement that the first and second terms on the right-hand side of this energy balance expression are given by $I^{2} R(t)$ and the time rate of change of $\frac{1}{2} L(t) I^{2}$, respectively, one obtains

$$
\begin{aligned}
V I & =I^{2} R+d / d t\left(\frac{1}{2} L I^{2}\right) \\
& =I^{2} R+L I d I / d t+\frac{1}{2} I^{2} d L / d t,
\end{aligned}
$$

where $V$ and $I$ are the applied toroidal voltage and the total toroidal current. In the stationary case $L$ can change only because of field diffusion. Dividing Eq. (6) by $I$ we obtain the circuit equation relating $V$ and $I$ for the above interpretation of $R$ and $L$, 


$$
\mathrm{LdI} / \mathrm{d} t+\frac{1}{2} \mathrm{IdL} / \mathrm{d} t+\mathrm{IR}=\mathrm{V}
$$

or alternatively,

$$
\frac{1}{2}\left[L \frac{d I}{d t}+d(L I) / d t\right]+I R=V
$$

It is now apparent that the $R$ and $L$ obtained using the conventional circuit equation, Eq. (2), does not clearly attach the ohmic heating to $R$ and the magnetic energy storage to $\mathrm{L}$ as does $\mathrm{Eq}$. (7). If $\mathrm{Eq}$. (2) is used one has either of two choices: (1) $\mathrm{L}$ is defined so that $\frac{1}{2} \mathrm{LI}^{2}$ is the field energy and $R$ defined so that the ohmic heating is given by $\left(R+\frac{1}{2} d L / d t\right) I^{2}$, or (2) $R$ is defined so that $I^{2} R$ is the ohmic heating and $L$ must be defined so that the rate of change of stored field energy is given by the unconventional formula $\mathrm{d} / \mathrm{dt}\left(\frac{1}{2} L \mathrm{I}^{2}\right)+\frac{1}{2} \mathrm{I}^{2} \mathrm{dL} / \mathrm{dt}$. It appears that the use of $\mathrm{Eq}$. (7) may be preferable since a clean association of ohmic heating and magnetic energy between the $R$ and $L$ parameters with the form of the energy terms is the same as they appear in standard circuit theory. (For the case of constant inductance, the two circuit equations, Eqs. (2) and (7), colncide, that is, L dI/dt $+I R=0$. ) The analogs of Eqs. (3) and (4) then become

$$
\begin{aligned}
& R(t)=V / I-\frac{1}{2} d L / d t-L(d I / d t) / I \text { and } \\
& L(t)=L(0)\left(\frac{I(0)}{I(t)}\right)^{2}+\frac{2}{I^{2}} \int_{0}^{t} I(V-I R) d t
\end{aligned}
$$

The time dependence of $\mathrm{L}(\mathrm{t})$ as given by Eq. (10) for the case of constant resistance and $V=0$ (that is, the equivalent circuit of $F i g . X-4(a)$ ) is shown in F+g. $\mathrm{X}-7$. This is to be compared with the corresponding result of Fig. $\mathrm{X}-6$ for the more conventional circuit equation, Eq. (1). One notes from Fig. X-7 that the use of Fq. (7) avoids the negative inductances which appeared in Fig. X-6. Again the use of Eq. (7) is perhaps preferable to Eq. (2). The use 


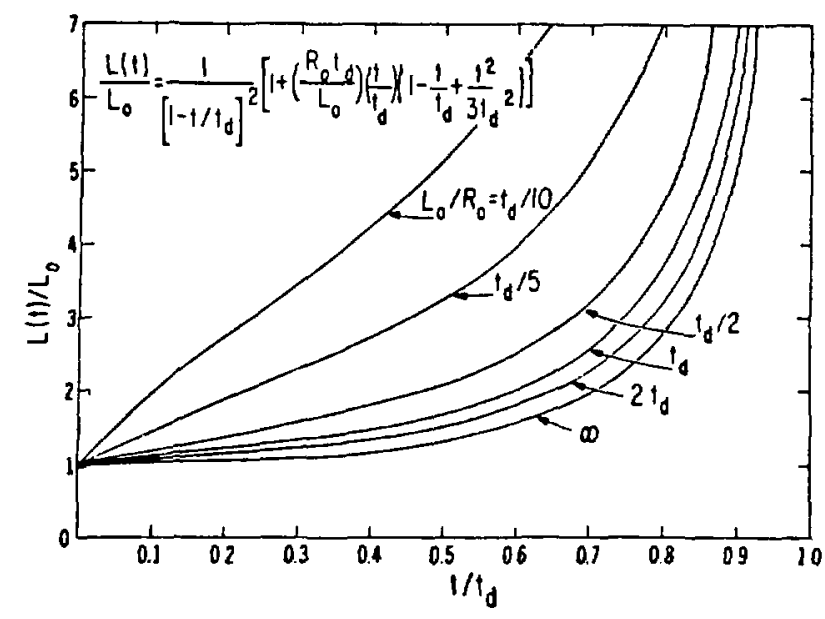

Fig. $\mathrm{x}-7$.

Same as Fig. X-6 except $L$ is to be used in Eqs. (7) or (8).

of Eq. (2) either mixes the rate of change of plasma energy between terms involving $R$ and $L$ or requires the use of a nonstandard formula for the rate of change of field energy.

B. Moving Conductor Case

When plasma motion is included in the model, there are further complications. It follows from Faraday's law that a moving thin resistive loop containing a voltage source $V$ carrying a current $I(t)$ can be described by Eq. (2) If LI is the flux linking the loop. If we multiply Eq. (2) by the current we obtain

$$
V I=d\left(\frac{1}{2} L I^{2}\right) / d t+I^{2} R+\frac{1}{2} I^{2} d L / d t
$$

The terms [starting on the left-hand side of Eq. (11)] can be identified as the power input, the rate of change of fleld energy, and the ohmic heating. This leaves the last term to represent the rate that the mechanical energy is changing due to the motion of the loop. In this approximation the distributed inductance due to field inside the conductor is negligible. For a plasma, however, the internal magnetic energy is important and can vary both because of motion and because the field diffuses. We have just shown that when diffusion is present it is clear that, if Eq. (7) is used for the moving 
plasma case and the magnetic energy is defined by the usual expression $\frac{1}{2} \mathrm{LI}^{2}$, $L$ is associated with stored fleld energy and hence the $I^{2} R$ of $E q$. (6) will of necessity include the rate of change of energy due to macroscopic plasma motion as well as ohmlc dissipation. The use of Eq. (7) has the property that L is clearly defined in terms of the fleld energy,

$$
L=\left.\frac{2}{I^{2}} \int_{\text {system }}^{\mid B}\right|^{2} /\left(2 \mu_{0}\right) d \tau
$$

and $R$ is given by total energy deposition rate (thermal plus nonthermal motion) into the plasma,

$$
R=\frac{1}{I^{2}} \int_{\text {system }}^{\vec{J}} \cdot \vec{E} d \tau
$$

Rather than try to justify Eq. (2) for distributed diffusing systems, we w111 use Eq. (7) and the defining Eqs. (12) and (13).

C. Numerical Estimates for the I,inear Decay in ZT-40

For the data in Fig. X-2 for the RFP operation of ZT-40, we have the lowest decay rate $\dot{I}=-I_{0} / t_{d}=\sim 1 \mathrm{kA} / \mu \mathrm{s}$. For a typlcal peak current of $I_{0} \simeq 400 \mathrm{kA}$, this corresponds a $0.4-\mathrm{ms}$ time interval $t_{d}$ for the current to decrease to zero. If we assume a constant inductance of $\mathrm{L}_{0} \simeq 1 \mu \mathrm{H}$ during the decay (this value is based on diffuse model profiles), the foregoing analysis using the circuit model of $\mathrm{F} 1 \mathrm{~g} \cdot \mathrm{X}-4(\mathrm{a})$ gives the total circult resistance as follows.

$$
R(t)=\frac{L_{o}}{E_{d}}\left(\frac{1}{T-E / t d}\right)=2.5\left(\frac{1}{T-2.5 t(m s)}\right) m \Omega
$$

Thus the total $R$ varies from $2.5 \mathrm{~m} \Omega$ to a very high value at the end of the decay. This is the sum of the plasma and the external circult resistance $R_{e}$. 
The approximate value of $R_{e}$, however, varies from $\sim 2.8 \mathrm{~m} \Omega$ at $400 \mathrm{kA}$ to $\sim 3.6 \mathrm{~m} \Omega$ at $200 \mathrm{kA}$. The variance is due to the spark gap switches that are

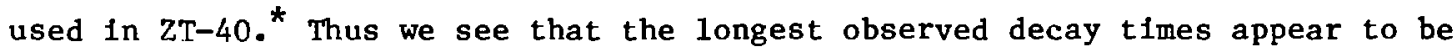
dominated by the external circult resistance, which increases with time. The single-turn-equivalent external inductance is $\sim 0.22 \mu \mathrm{H}$ and has 1ittle effect in the above argument. Thus the decay time for the longest decays in ZT-40 appears to be limited by the external resistance. The shorter decays 1mply $R_{p} \sim 3 m \Omega$ or greater. One must bear in mind that $R$ is an effective resistance which includes plasma gross motion or turbulence when f.t exists. We can, on the other hand, assume a constant $R(t)$ and look at the $L(t)$ variation to give She linear decay. From the graph of Fig. X-6 we see that the inductance of the piasma would have to vary nearly an order of magnitude to explain the observed decay. This seems unlikely. A variation in both $R_{p}(t)$ and $L_{p}(t)$ during the decay is certainly a possibility. The increase in decay time (Fig. X-3) for the RFP field configuration is belleved to be a manifestation of the lower effective resistance due to the better confinement properties of the RFP. A change of inductance cannot be ruled out as having an effect, however.

The simple method above becomes limited when the coupling of the $v_{\theta}$ circuit is included. A general more sophisticated scheme which has been proven successful in matching waveforms of the $7 T-40$ over the whole discharge is discussed in Chapter XX.

₹ The $R_{e}$ and $I_{e}$ numbers were obtained from $R$. Gribble. 
CHAPTER XI

THOMSON SCATTERING ELECTRON TEMPERATURE MEASUREMENTS

by

A. Haberstich, K. B. Freese, K. A. Klare, and R. G. Watt

The electron temperature of $\mathrm{ZT}-40$ has been measured with a single-point, single-time Thomson scattering diagnostic. Data have been obtained in the stabilized (nonreversed), self-reversed, and aided-reversal modes. All measurements have been performed on the minor geometric axis of the discharge.

I. DIAGNOSTIC

A general view of the diagnostic is shown in Fig. XI-1. The $6943 \mathrm{~A}$ incident beam is produced by a Holobeam model 6081 ruby laser generating 5 Joule pulses with a nominal divergence of 1.5 mrad. The laser is located in

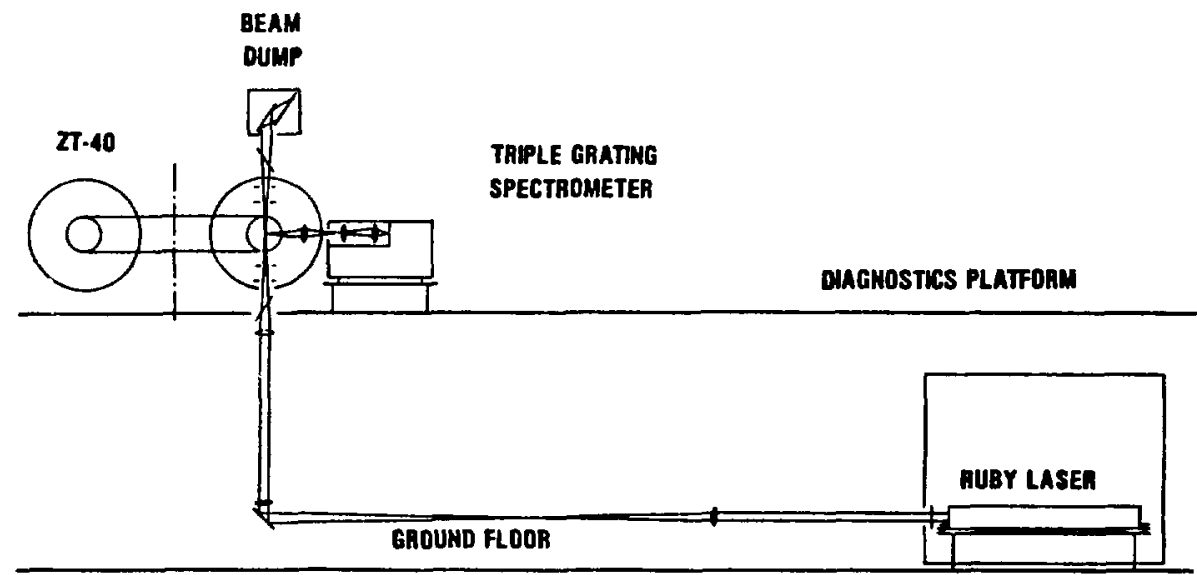

Fig. XI-1.

ZT-40 single-point Thomson scattering diagnostic. 
a shielded room, on the ground floor of the ZT-40 fac1lity, $15 \mathrm{~m}$ away from the experiment. Control and monitor signals to and from the laser room are transmitted by optical links to prevent electrical interference. The laser beam is transferred to the experimental platform by two relay lenses, a focusing lens, and two turning prisms, and traverses the discharge chamber through two Brewster-angle windows.

Figure XI-2 shows the scattering geometry. The focusing lens is movable, to allow the incident beam to be focused at any chosen position along the 40-cm minor diameter of the discharge chamber. Nine 5.08-cm (2 1nches) diameter sapphire windows are sealed in the wall of the ceramic liner to allow the light scattered by the plasma to be observed at various radial positions. The nature of the ceramic chamber makes the use of a viewing dump impractical.

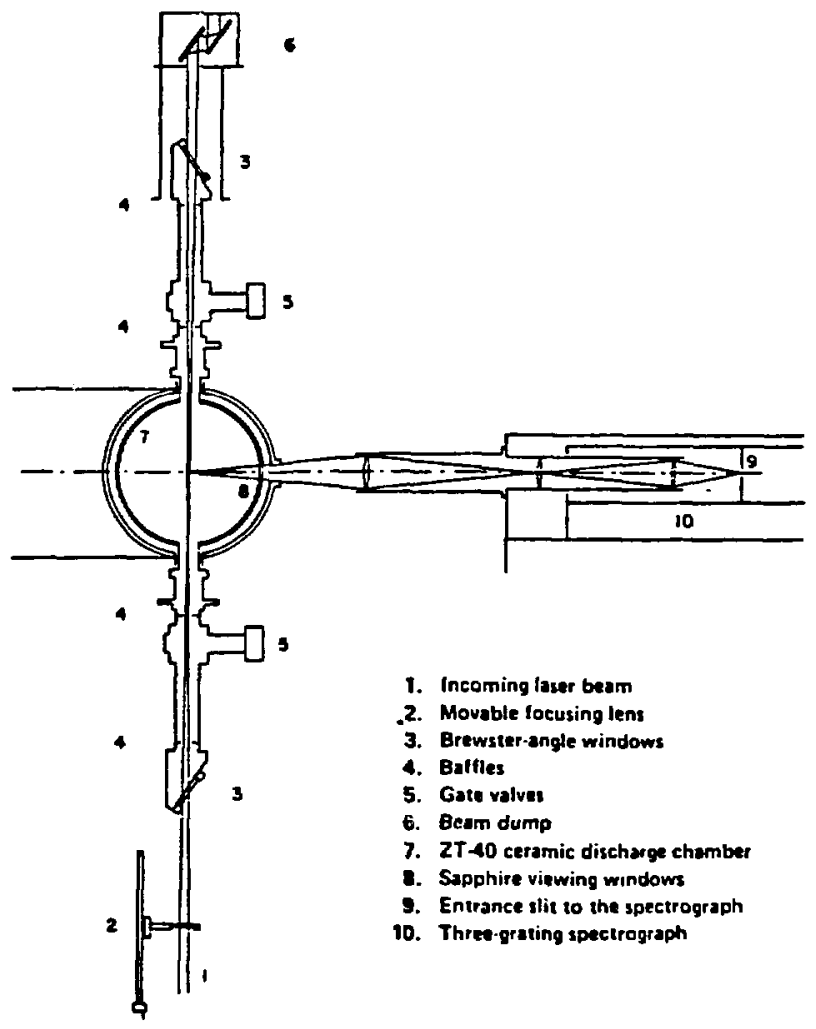

F1g. XI-2.

Scattering geometry. The Brewster-angle windows and the beam dump are rotated $90^{\circ}$ for clarity. 
Two light-gathering optics have been used to focus the scattered light on the 1-by-10-mm entrance slit of the spectrum analyzer. The first geometry, consisting of three simple lenses, as shown in the figure, gave a usable plasma scattering area of 2 by $20 \mathrm{~mm}$. This arrangement was later replaced by a single lens made of two aerial camera objectives mounted back to back. This configuration gave a scattering area of 1 by $10 \mathrm{~mm}$. The reason for the change to a higher quality optic was to allow a more uniform illumination of the first grating of the spectrometer.

The spectrum analyzer is a triple-grating instrument used previously on the Scylla IV-P theta pinch experiment, and modified electrically for use on 2T-40. The principle of operation of this instrument, shown schematically in FIg. XI-3, has been described in detail elsewhere.1,2 The scattered light entering sitt $S_{1}$ is dispersed by the grating $G_{1}$, spectrally filtered by the mask $M$, recombined at the grating $G_{2}$, and focused on the slit $s_{2}$. The spectrum of the light passing through $S_{2}$ is dispersed by the grating $G_{3}$, and focused on an array of 10 light guldes connected to 10 photomultipliers. The advantage of the three-grating concept is to provide effective rejection of the unwanted 6943 \& radiation. This particular spectrograph has an f-number of 5 , a dispersion of $20 \AA / \mathrm{mm}$, and a theoretical $6943 \AA$ rejection ratio of $10^{-12}$. The width of the light guides increases exponentially from $1 \mathrm{~mm}$ on channel 1 to $10.6 \mathrm{~mm}$ on channel 10. This arrangement is Ideal to look at the blue side of kilcvolt spectra.

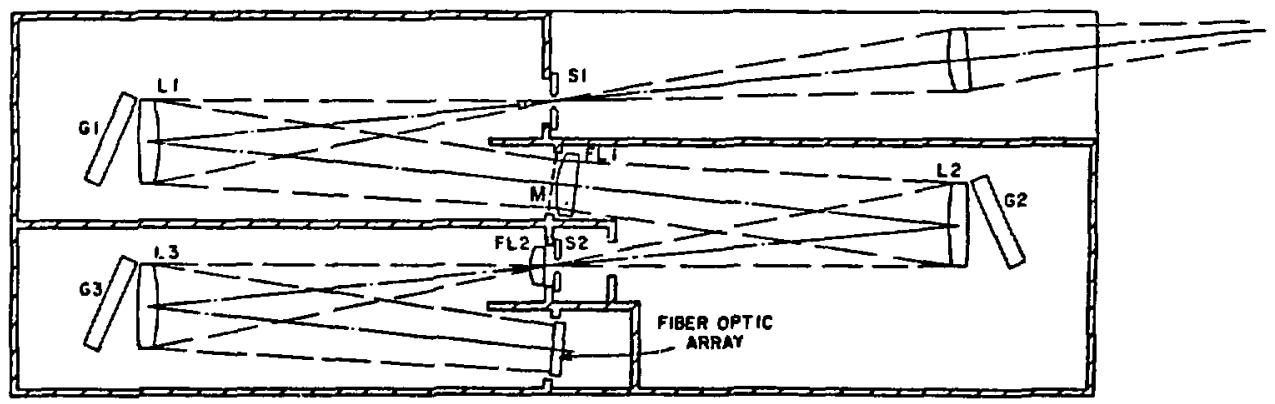

Fig. XI-3.

Triple-grating spectrometer. 
Because of the lower ZT-40 temperatures, only five of the photomultiplier channels have been activated for the reversed-field pinch measurements. The locations of these channels with respect to hypothetical 50 and $100 \mathrm{eV}$ spectra are shown in Fig. XI-4. Two configurations have been used: (1) configuration A looking at the blue side, and (2) configuration B looking at both sides of the Maxwellian. The purpose of the second geometry is to monitor the symmetry of the scattering spectrum.

The photomultipliers are multialkal1, 12-stage tubes, RCA type 8852 or C31000A with quantum efficiencies of about $4 \%$ at $6943 \AA$. The original photomultiplier assemblies have been modified to improve magnetic shielding and to turn on the tubes microseconds before the firing of the laser. This gating is done to avold depletion of the high-voltage supply due to background

\section{DETECTOR CHANNEL POSITIONS}

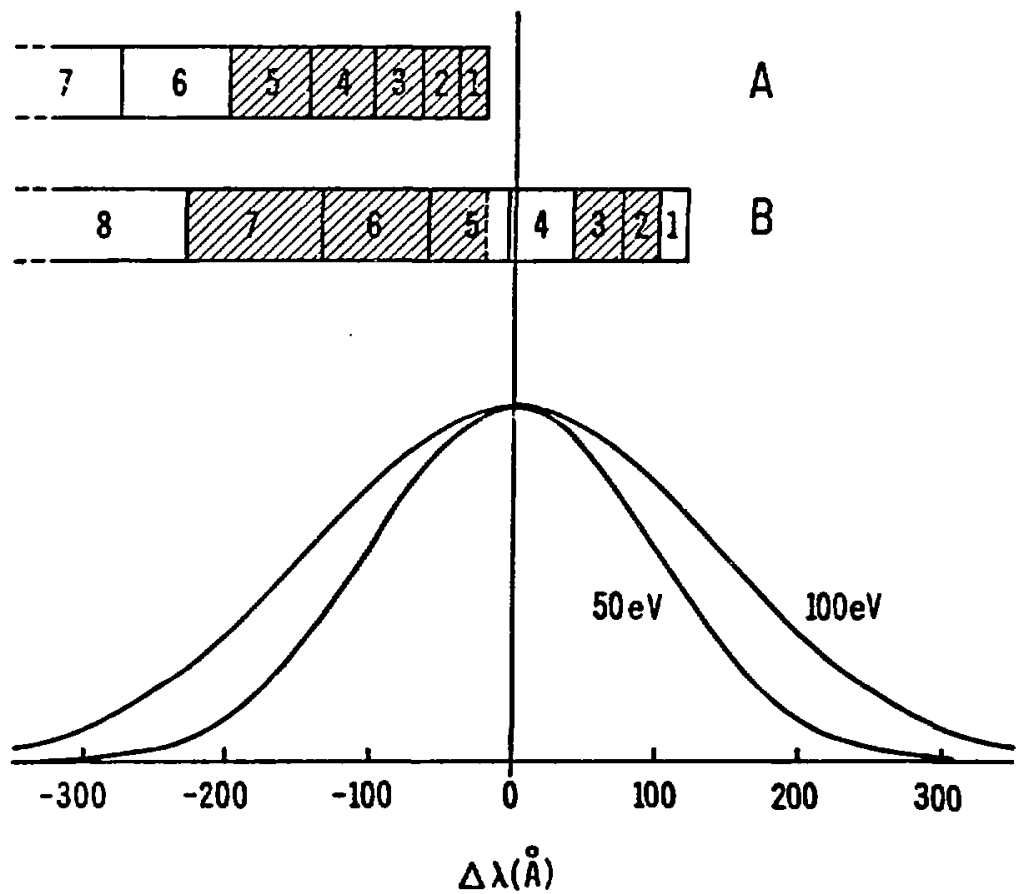

Fig. XI-4

Photomu1tiplier channel configurations: (A) sampling the blue side, and (B) sampling both sides of the scattered spectrum. For comparison, 50and 100-eV Maxwellians are shown. 


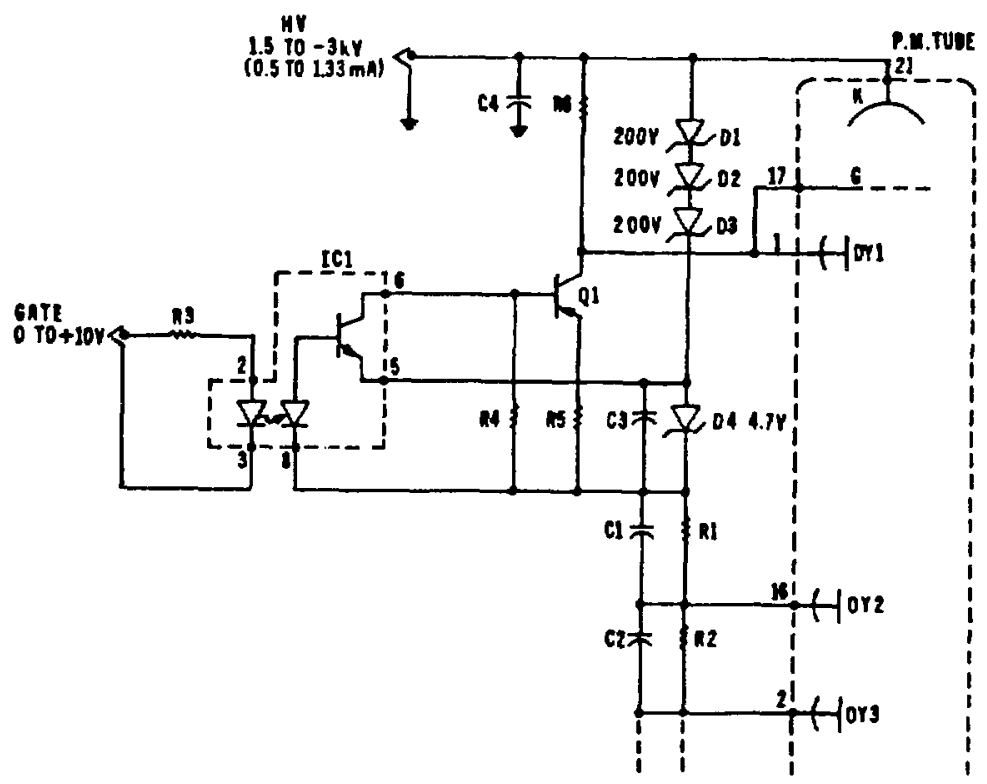

Fig. XI-5.

Photomultiplier gating circuit.

radiation from the discharge. A schematic diagram of the photomultiplier base is shown in Fig. XI-5. The turn-on time of the gate is about $5 \mu$, whereas the less important turn-off time is a few hundred microseconds.

The photomultiplier outputs are recorded simultaneously by oscilloscopes and by charge-sensitive LeCroy model 2249 analog-to-digital converters. A schematic diagram of the computerized data acquisition system is shown in Fig. XI-6. The system reads the five photomultiplier signals and a monitor signal during and shortly after the laser pulse. The second reading is subtracted from the first to determine the net scattering signal. The monitor signal, generated by an ITT FW114A fast photodiode sampling the $6943 \AA$ laser beam, gates the A/D converters and is used to normalize the data. Following the ZT-40 discharge, another reading takes place to record the base Iines. The interval counter provides a record of the timing of the measurement with respect to the beginning of the discharge.

The primary calibration of the instrument is done with a quartz lodine lamp. The light from the filament is converted into light pulses by a fast-rotating slit. This calibration, performed at least once a day, is used for the data analysis. A secondary calibration is done with a xenon strobe 


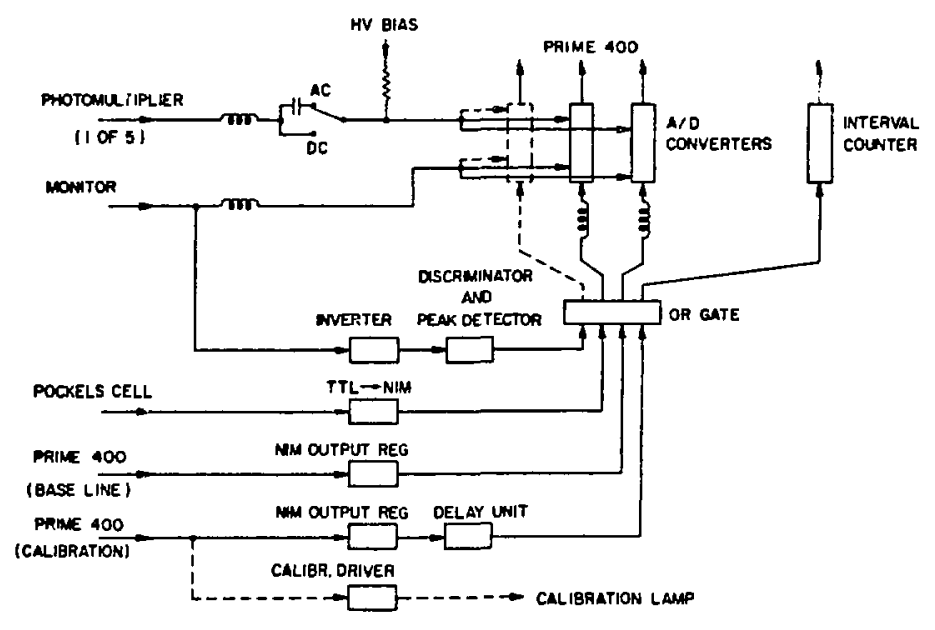

Fig. XI-6.

Computerized data acquisition system. Dashed lines linolcate proposed mudifications.

light located inside the spectrometer, near the entrance slit. The strobe lamp is fired 3 us after the laser pulse, and the pictures taken with the oscilloscopes can be checked to detect changes in sensitivity, if any, during the discharges.

Temperatures are obtained in the usual way by fitting the photomultiplier data to a Maxwellian distribution. Because of the relatively low electron temperatures, relativistic corrections are ignored. The computer and hand analyses differ in the sense that the computer code fits the data to a Gaussian function of the wavelength, whereas the hand analysis fits the logarithm of the data to a linear function of the square of the wavelength. There is another difference in that the computer takes the finite widths of the photomultiplier channels into account by performing a convolution with the calculated spectral distribution. The hand analysis assumes infinitely narrow channels. Reduction of the same data with the two programs produces differences that are negligible, when compared to other uncertainties.

The useful range of the diagnostic is limited by the noise caused by photon statistics on the background radiation from the plasma and from the wall of the discharge chamber. Whereas the intensity of the scattered light is proportional to the density of the plasma, the statistical nolse amplitude varies only with the square root of the background light. The resulting 
degradation of the signal-to-nolse ratio at low plasma density is accentuated by the fact that background radiation around $6943 \AA$ does not decrease significantly at lower fill pressures. A comparison of computer and scope acquired data shows good agreement at f111 pressures of $10 \mathrm{mtor} r$ and above. At lower pressures, discrepancies develop because of the noise problem. The results presented here have, therefore, been obtained or confirmed by hand on the basis of scope data.

\section{MEASUREMENTS}

The temperatures have all been measured on the minor geometric axis of the experiment. Each temperature plot is the result of at least one dedicated ZT-40 run, and almost all the data taken during this run are shown. Each data point represents one measurement during one discharge. The error bars are calculated in two ways. The first error is a propagated error proportional to the uncertainty in the raw data. The second is a chi-squared error describing the goodness of the fit with the Maxwellian distribution. The larger of the two errors, usually the chi-squared error, is plotted in the figures.

Figure XI-7(a) shows the result of an electron temperature measurement in the stabllized (nonreversed) mode of operation of $\mathrm{ZT}-40$. The data are plotted as functions of deuterium $\mathrm{f} 111$ pressure. Typlcal toroldal current, average toroldal fleld, and toroldal fleld at the wall are shown In Fig. XI-8 as functions of time. The toroldal current is turned on at time $50 \mu \mathrm{s}$ and peaks and is passively crowbarred at $150 \mu \mathrm{s}$. The toroldal field is turned on at time zero, and is passively crowbarred at the onset of the toroidal current. The anticlpated drop of the toroldal field at the wall, between 50 and $140 \mu \mathrm{s}$, is due to the compression of the fleld on axis under constant toroldal flux conditions.

FIgure XI-7(b) shows data similar to Fig. XI-7(a), but taken approximately 4 months later. The toroldal current is now power crowbarred, but with a voltage low enough to approximate the earlier passive crowbar operation. Also, the earlier spark gap switches are replaced by ignitrons. The current, flux, and fleld waveforms remain similar to the traces shown in Fig. XI-8. Figure XI-7(a) is obtained with the photomultiplier configuration $A$ and Fig. XI-7(b) with configuration $B$. 

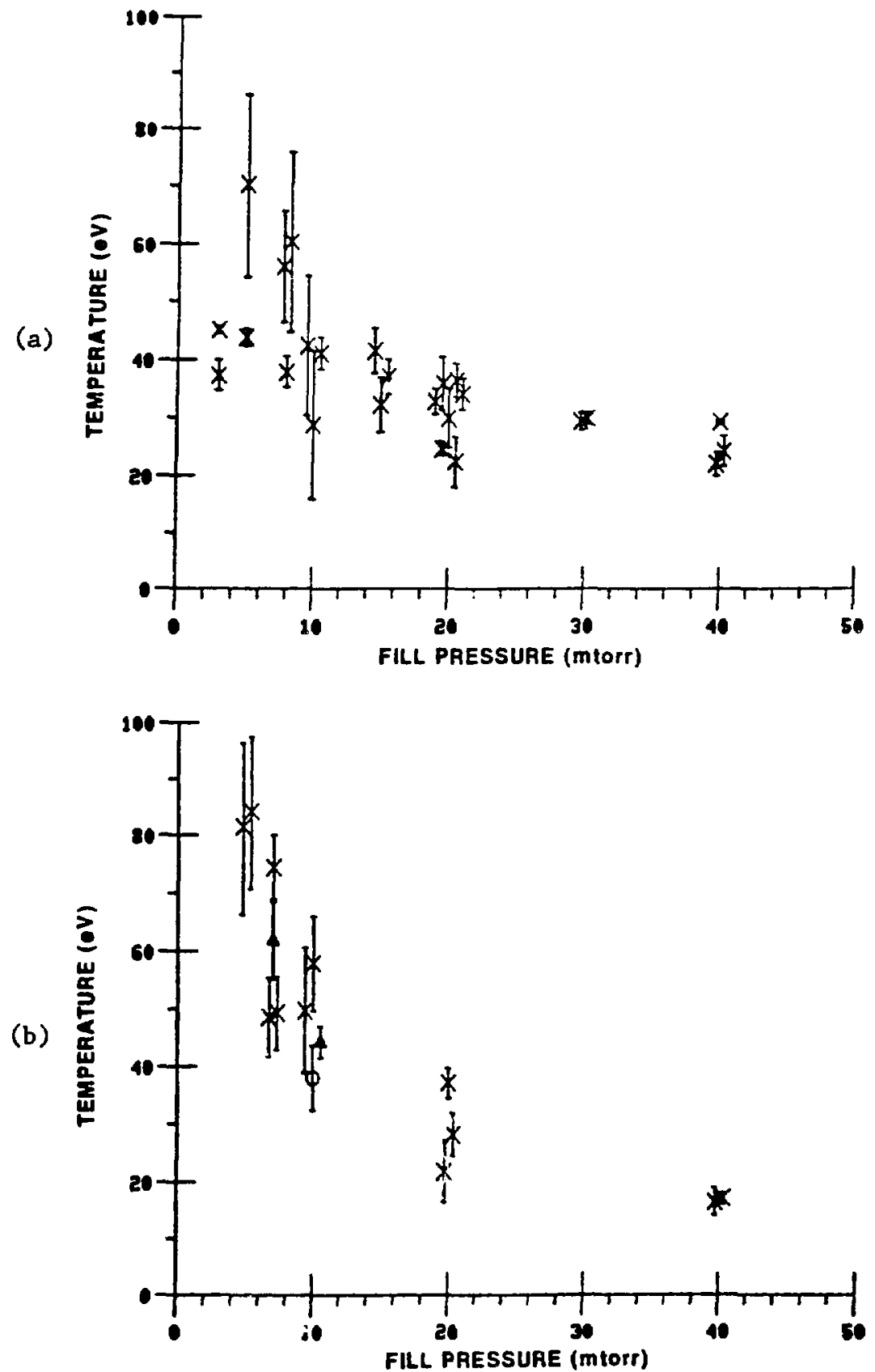

F1g. XI-7.

Electron temperatures as functions of fill pressure. (a) April 17, 1980, configuration A, time $150 \mu \mathrm{s}$, (b) August 11, 1980, configuration B, crosses $150 \mu \mathrm{s}$, circle $125 \mu \mathrm{s}$, triangles $180 \mu \mathrm{s}$. Peak toroidal current, $450 \mathrm{kA}$. 
Shot 1661
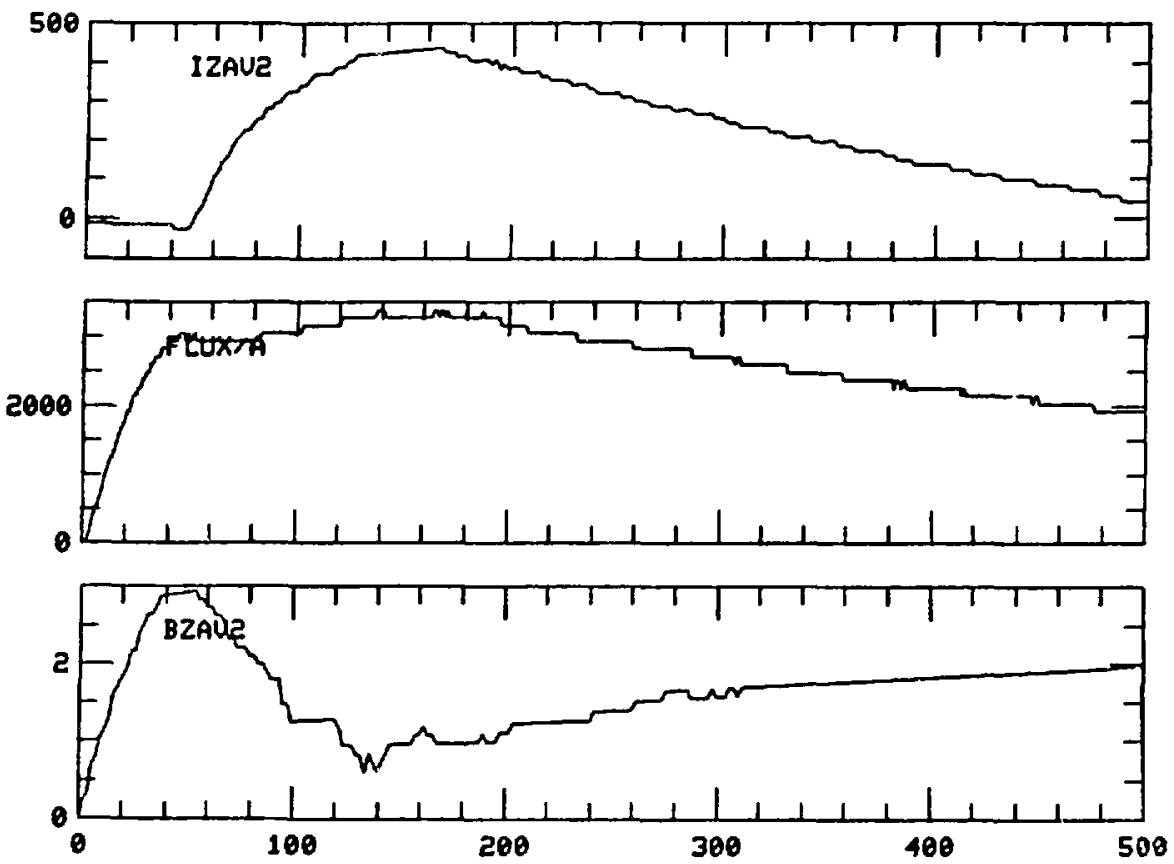

F1g. XI-8.

Stabilized pinch. Toroldal current (kA), average toroidal fleld (G), and toroidal field at the wall $(\mathrm{kG})$, as functions of time ( $\mu s$ ).

The temperatures observed with the new photomultiplier wavelengths are comparable to the earlier measurements. Combining the results of Figs. XI-7(a) and (b), one concludes that the axial temperature at peak toroidal current in this mode is 20 to $25 \mathrm{ev}$ at the high end of the fill pressure range. The temperature peaks at around $80 \mathrm{eV}$ at 5 mtorr fill and then drops at lower density.

The corresponding relative electron densities, obtained from the area under the curve of the scattered spectrum, are shown in Figs. XI-9(a) and (b). Because of the 6943 \& mask, an absolute callbration of the spectrometer has not been done. The density scales are arbitrary and are different in the two figures. Figure XI-9(b) shows a linear increase of the central density with f111 pressure.

F1gures $X I-10(a)$ and (b) show electron temperatures as functions of peak value of the toroldal current. These results are again obtained in the stabilized (nonreversed) mode, at peak toroldal current, at $150 \mu 8$. The 

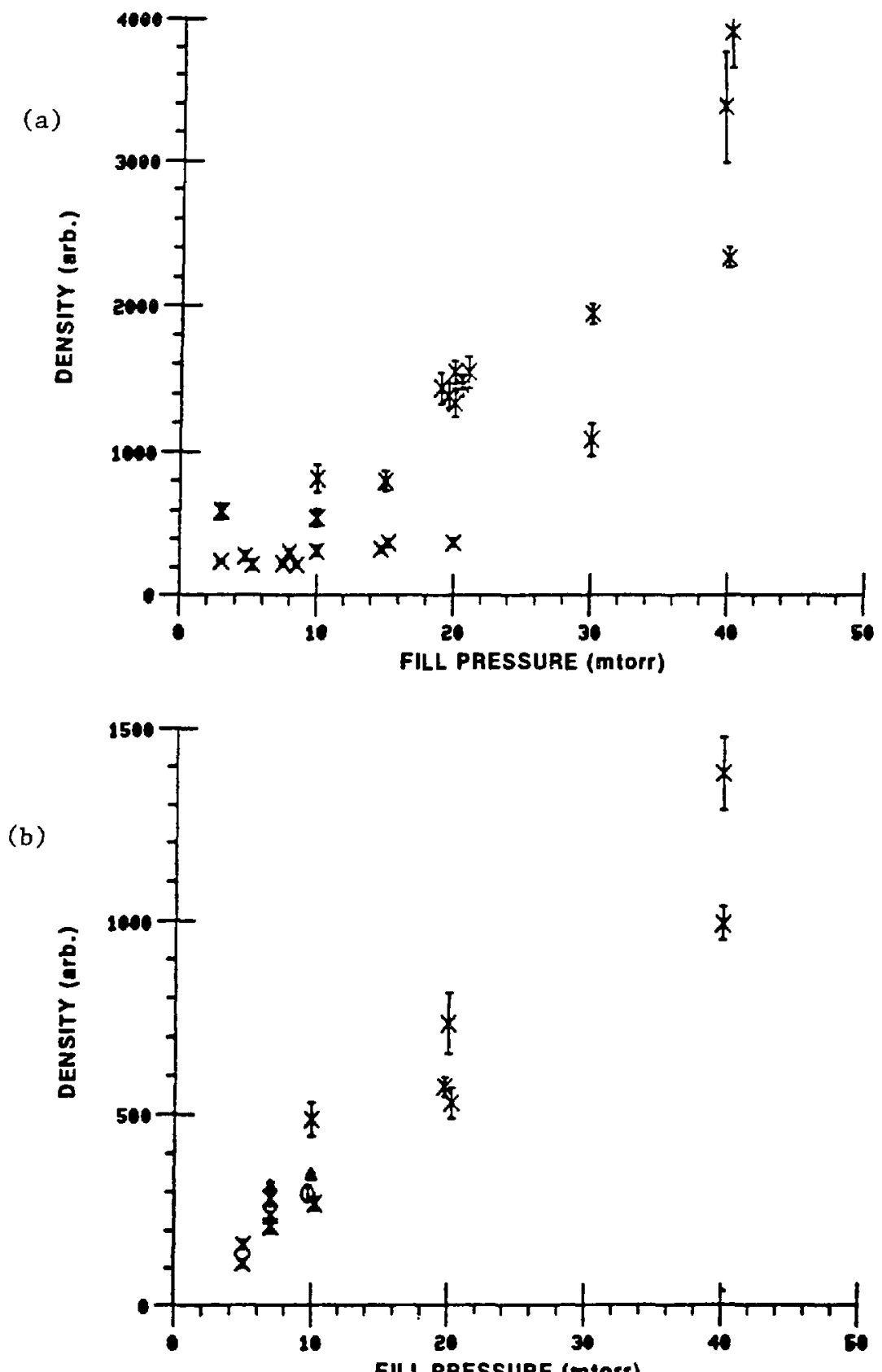

FILL PRESSURE (mtorr)

Fig. XI-9.

Stabilized pinch. Relative electron densities corresponding to the data of F1g. XI-7. 

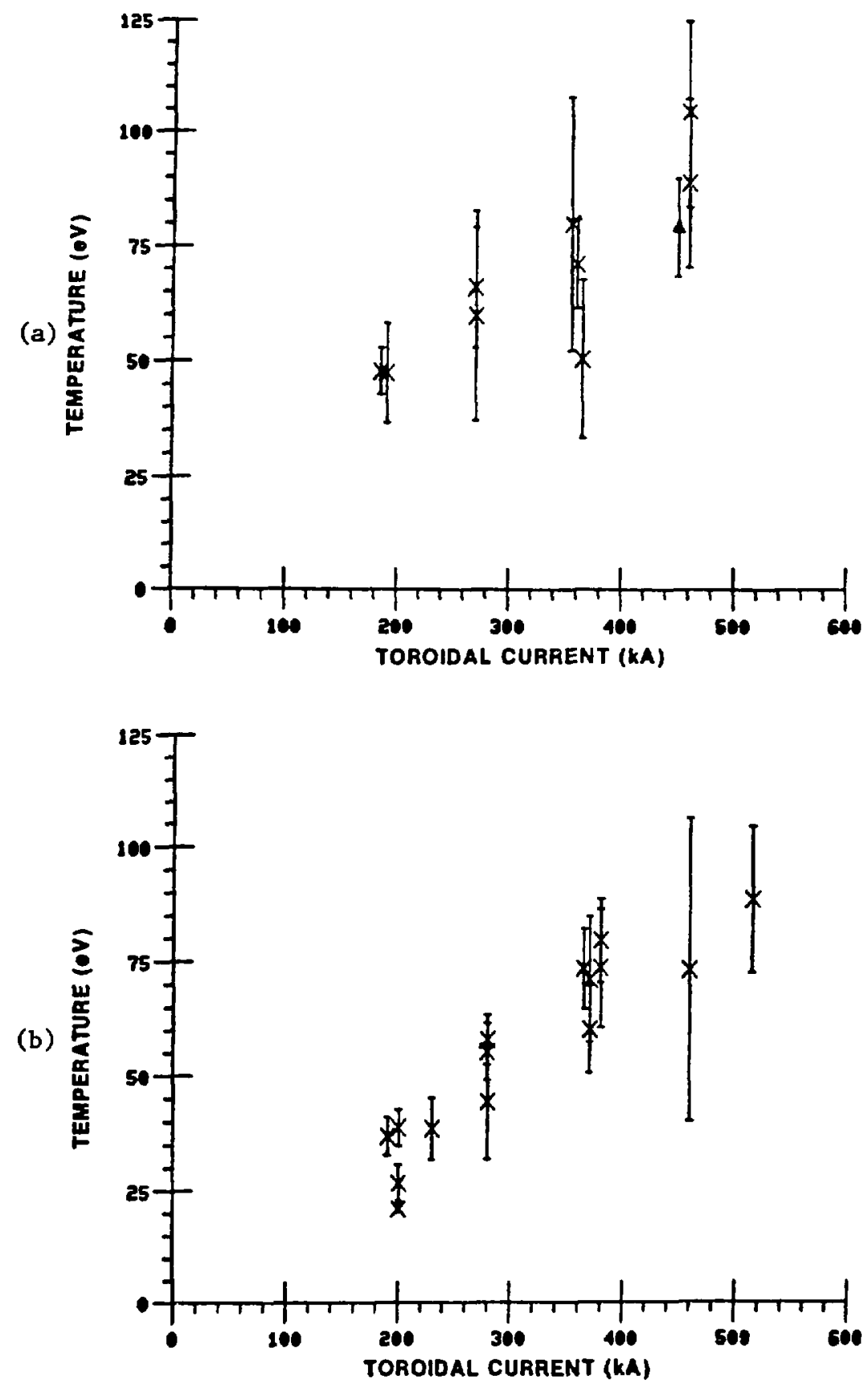

F1g. XI-10.

Stabilized pinch. Electron temperatures as functions of peak toroidal current: (a) 7-mtorr fill and (b) 10-mtorr fill; time, $150 \mu \mathrm{s}$. The triangle in XI-10(a) indicates a higher theta discharge. 
deuterium fill pressure is 7 mtorr in $71 \mathrm{~g}$. XI-10(a) and 10 mtorr in Fig. XI-10(b). The charging voltages of the toroldal and poloidal banks are varied simultaneously to maintain an approximately constant value of theta. Theta is defined as the ratio of the poloidal field at the wall over the average torcidal field. The measured temperatures are seen to increase from 25 to $40 \mathrm{eV}$ at $200 \mathrm{kA}$ to $85 \mathrm{eV}$ at $500 \mathrm{kA}$. Temperatures obtained at 5 mtorr deuterium fill pressure, In Fig. XI-11(a), show the same trend, although the error bars are much larger. The 5-mtorr f 111 density measurements, shown on a relative scale in $\mathrm{Fig}$. XI-11(b), indicate an increase in central density as a function of peak toroidal current. In other words, the temperature does not increase at the expense of a loss of central density. Figures XI-10 and 11 are obtained by looking only at the blue side of the scattered spectrum.

Temperature measurements have also been carried out in the self-reversed mode of operation of 2T-40. This mode is obtained by lowering the initial toroidal magnetic field. The compression of the toroidal field on axis is then accompanied by a reversal of the toroldal field at the wall. This spontaneous reversal has to do with the tendency of reversed-field pinches to relax into a low energy state. 'Waveforms measured during this run are shown in Fig. XI-12. The toroldal current peaks at about $150 \mu \mathrm{s}$, and the reversal is seen to last, In this case, from time 100 to $260 \mu \mathrm{s}$. Figure XI-13 shows the electron temperature as function of time for a peak toroidal current of $480 \mathrm{kA}$ and a deuterium $\mathrm{f} 111$ pressure of 8 mtorr. The measurements have been made at 150, 250, and $350 \mu \mathrm{s}$ in the discharge. As in some of the other figures, the data are slightly spread out along the abscissa for clarity.

A third mode of operation of $2 T-40$ is the alded-reversal mode. Alded reversal is obtalned by choosing approximately the same inftial value of the toroldal magnetic fleld as in the stabilized mode, and by delaying the crowbar on the toroldal field beyond the peak of the toroldal current. A set of waveforms is shown In FIg. XI-14. Note that the onset of the toroidal fleld takes place at 30 us rather than at time zero during this run. This type of programing is similar to so-called pitch programming, where the desired magnetic pitch profile is convected into the plasma from the wa11. Typical of the afded-reversal mode is the rapid drop of toroldal magnetic flux caused by the natural oscillation of the torofdal field capacitor bank. This drop is checked at $140 \mu s$, with the application of a passive crowbar. The toroldal current was both passively and actively crowbarred during this run. 

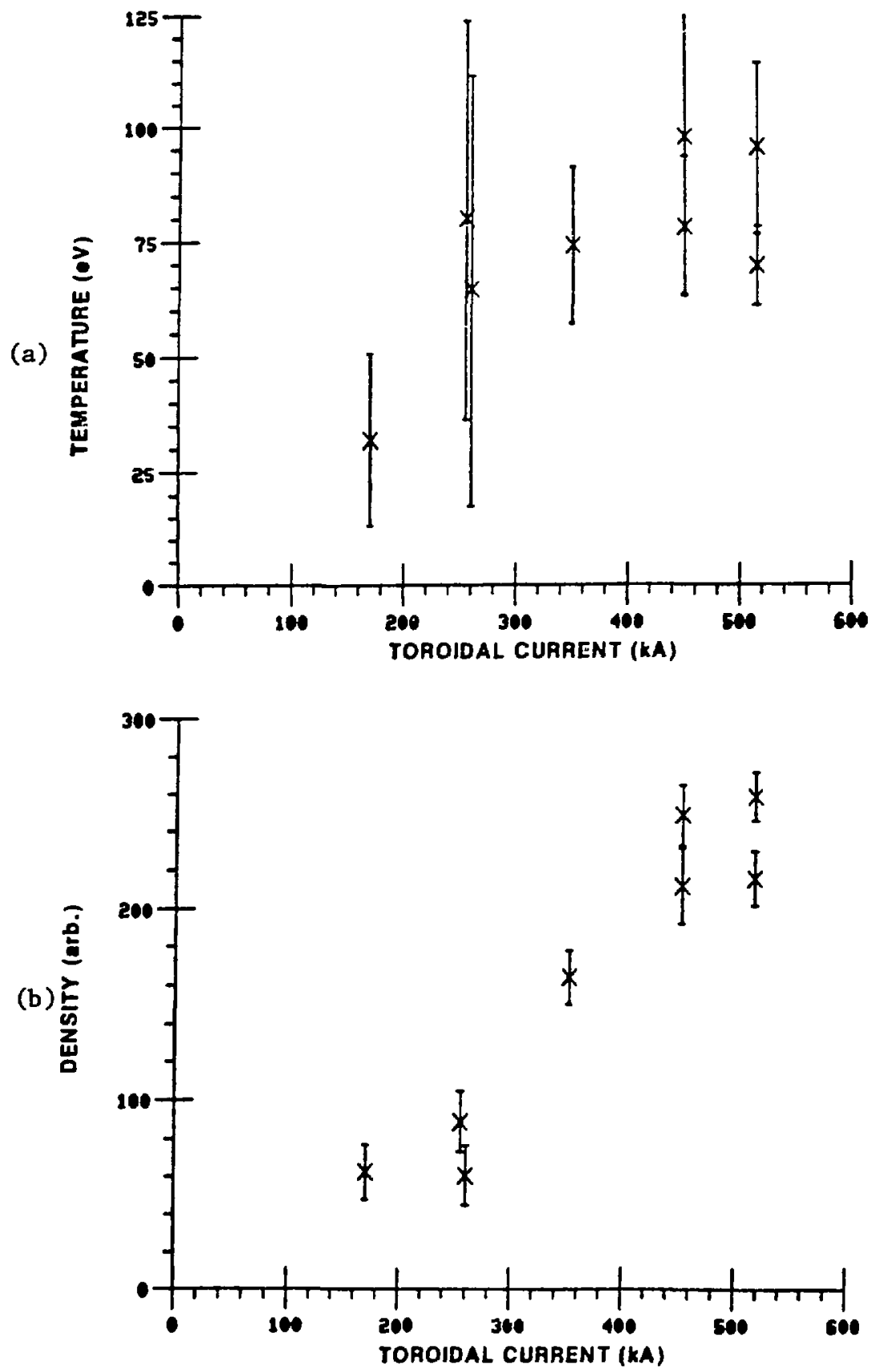

Fig. XI-11.

Stabilized pinch. (a) Electron temperature and (b) relative electron density as functions of peak toroldal current. F111 pressure--5 mtorr, t1me--150 us. 


\section{Shot 2401}
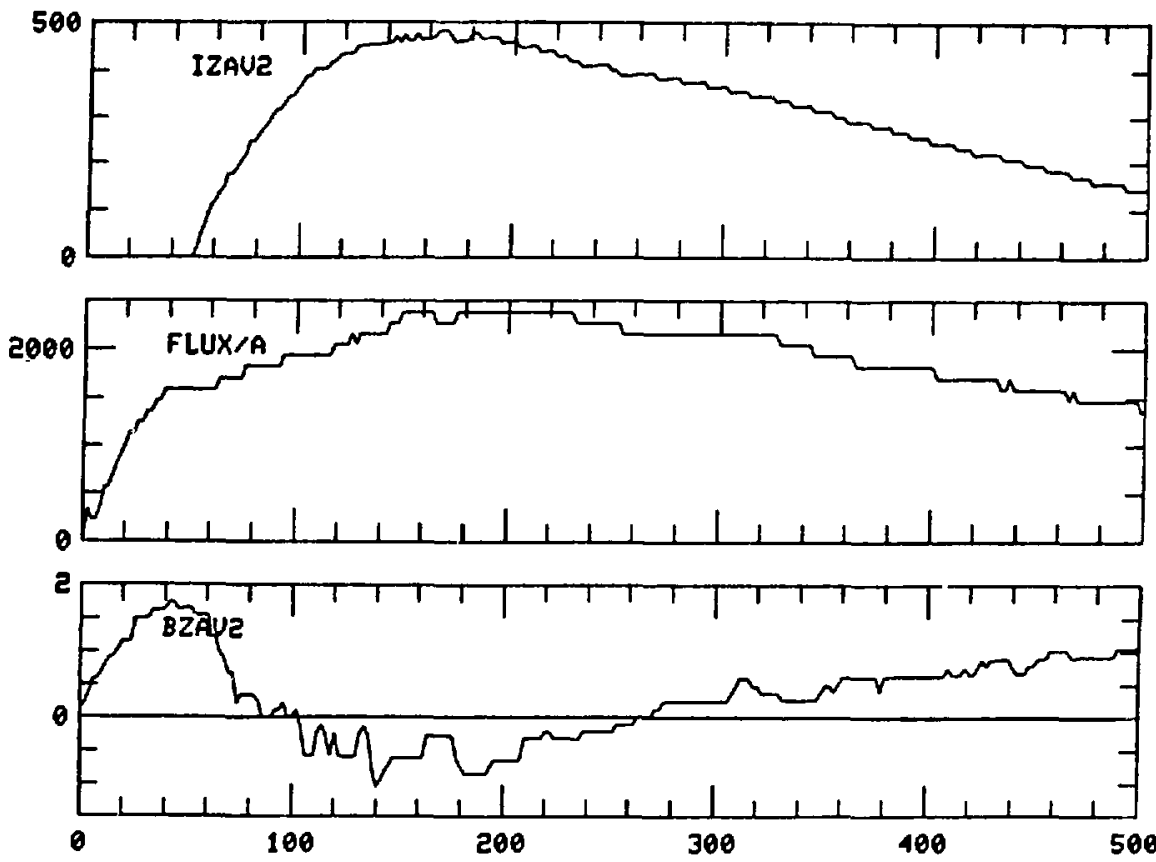

Fig. XI-12.

Self-reversed pinch. Same display as in Fig. XI-8.

Figures $X I-15(a)$ and (b) show measured temperatures as functions of time for peak values of the toroidal current of 480 and $280 \mathrm{kA}$. The deuterium fill pressure is 7.5 mtorr in both cases. The maximum measured electron temperature is about $90 \mathrm{eV}$ at $480 \mathrm{kA}$ and $70 \mathrm{eV}$ at $280 \mathrm{kA}$. Note that the temperature peaks in both cases approximately $50 \mu \mathrm{s}$ after peaking of the toroldal current. These results were obtained with the photomultiplier configuration $B, 100 k i n g$ at both sides of the spectrum.

All the data discussed so far have been measured with the ZT-40 experiment being run in a four-feed configuration. Data were also taken during the brief period when the voltage producing the toroldal current was applied at a single feed point. This mode of operation lengthens the rise time of the toroidal current from 100 to $250 \mu \mathrm{s}$. Typical toroldal current, average toroldal fleld, and toroldal field at the wall are shown in FIg. XI-16, for a $f 111$ pressure of 20 mtorr. 


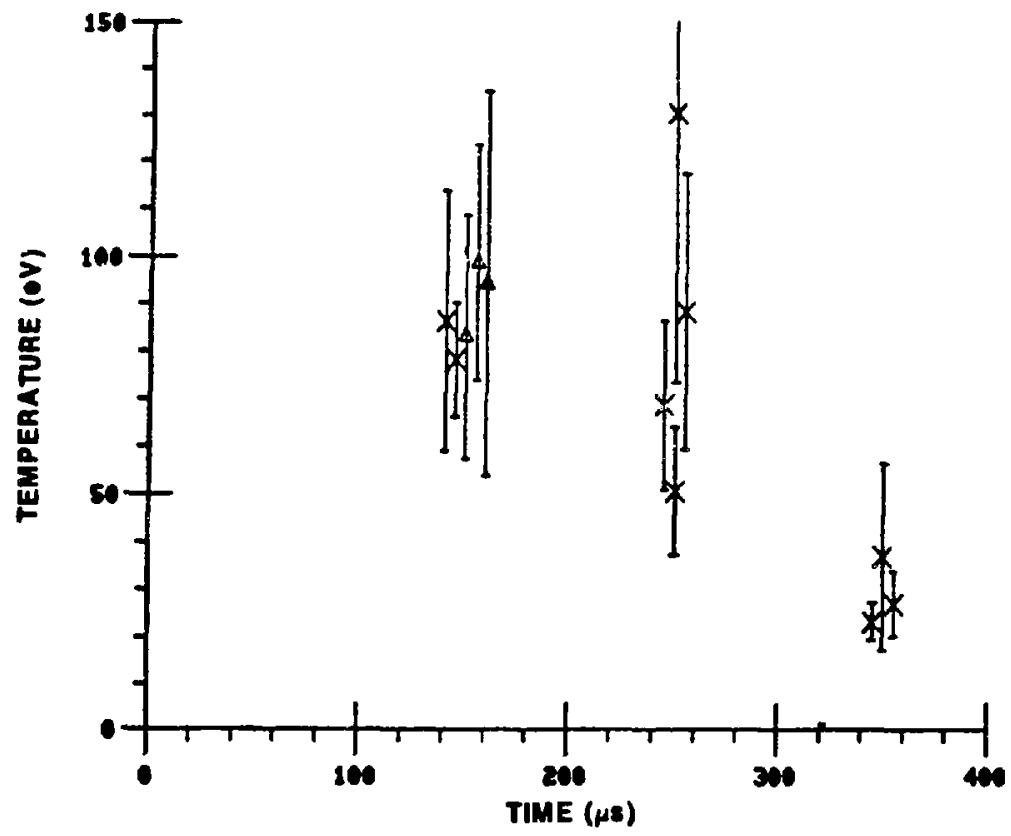

Fig. XI-13.

Self-reversed pinch. Electron temperature as function of time. Eightmtorr fill, peak toroldal current: crosses $480 \mathrm{kA}$, triangles $350 \mathrm{kA}$, same value of theta.

Corresponding electron temperature readings are reported in Fig. XI-17, as functions of time in the discharge. The peak toroidal current during this 20 -mtorr deuterlum run was $400 \mathrm{kA}$. The photomultipliers were stationed according to configuration $A$.

\section{DISCUSSION}

It was difficult, in some Instances, to match the data to a Maxwellian distribution. Channel 1 often had to be 1gnored. In certain cases, such as in Fig. XI-7(a), channel 2 tended to be low and had to be left out to avold an overestimate of the temperature. The problem occurred when looking at the blue side of the spectrum (configuration A In Fig. XI-4).

The response of channe1 1 is affected by the proximity of the $6943 \AA$ mask, and part of the problem with this channel may have been due to focusing difficulties at the plane of the mask. The discrepancy with channel 2 usually happened at fill pressures below 10 mtorr, mostly at high electron 
Shot 2711
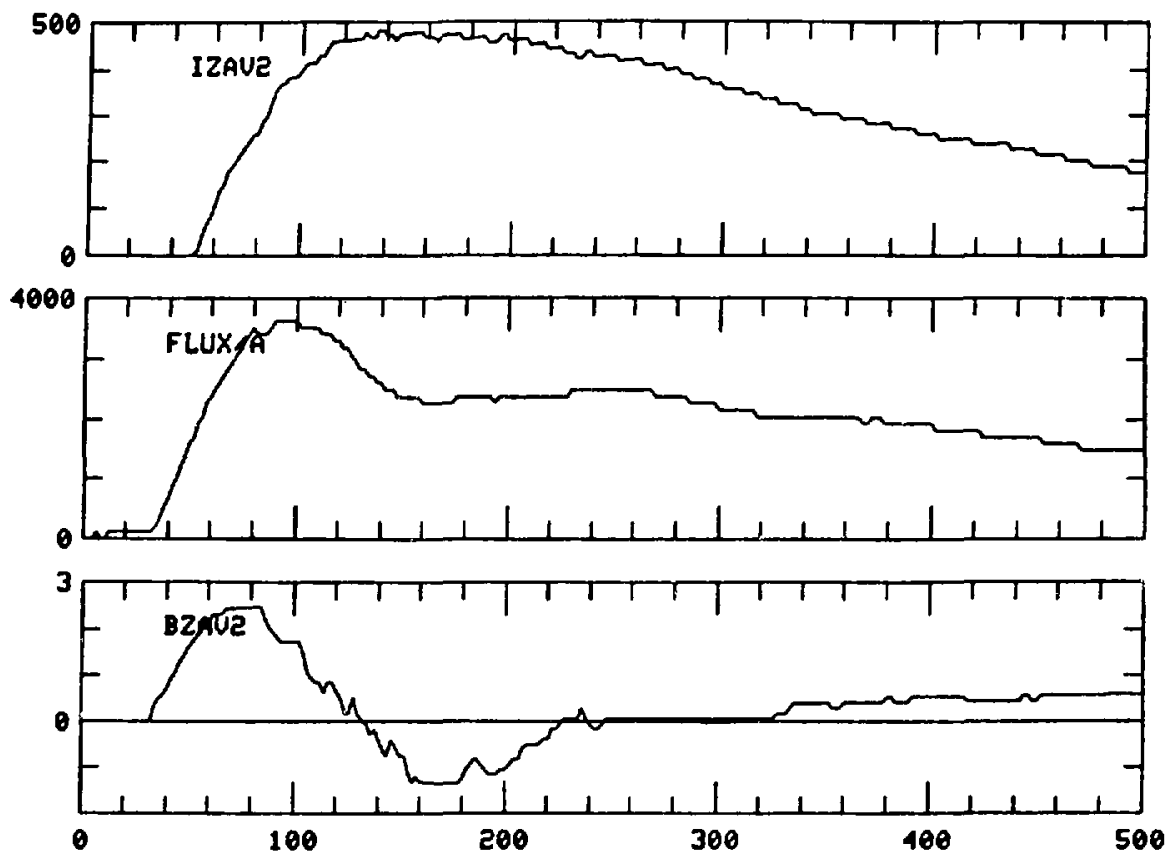

F1g. XI-14.

Aided reversal. Same display as in Fig. XI-8.

temperatures. Whether this problem was due to the plasma or to the diagnostic has not been resolved.

The possibility of an asymmetry of the scattered spectrum was investigated by looking at both sides of the spactrum (configuration $B$ in Fig. XI-4). At temperatures of the order of $50 \mathrm{eV}$ or less, the spectral distribution appeared to be symmetric. Whether this held true at higher temperatures was difficult to ascertain without extending the measurement further into the red. This was not done because of the increasing widths of the existing light guides. To explore the possibility of a shift due to the azimuthal component of the plasma current density, the direction of the toroldal magnetic field was temporarily reversed. This had no noticeable effect on the symmetry of the spectrum.

As mentioned earlier, the photomultiplier tubes were gated, and the secondary calibration was timed to check the sensitivity of the photomultiplier channels within microseconds of the laser pulse. Also, the 

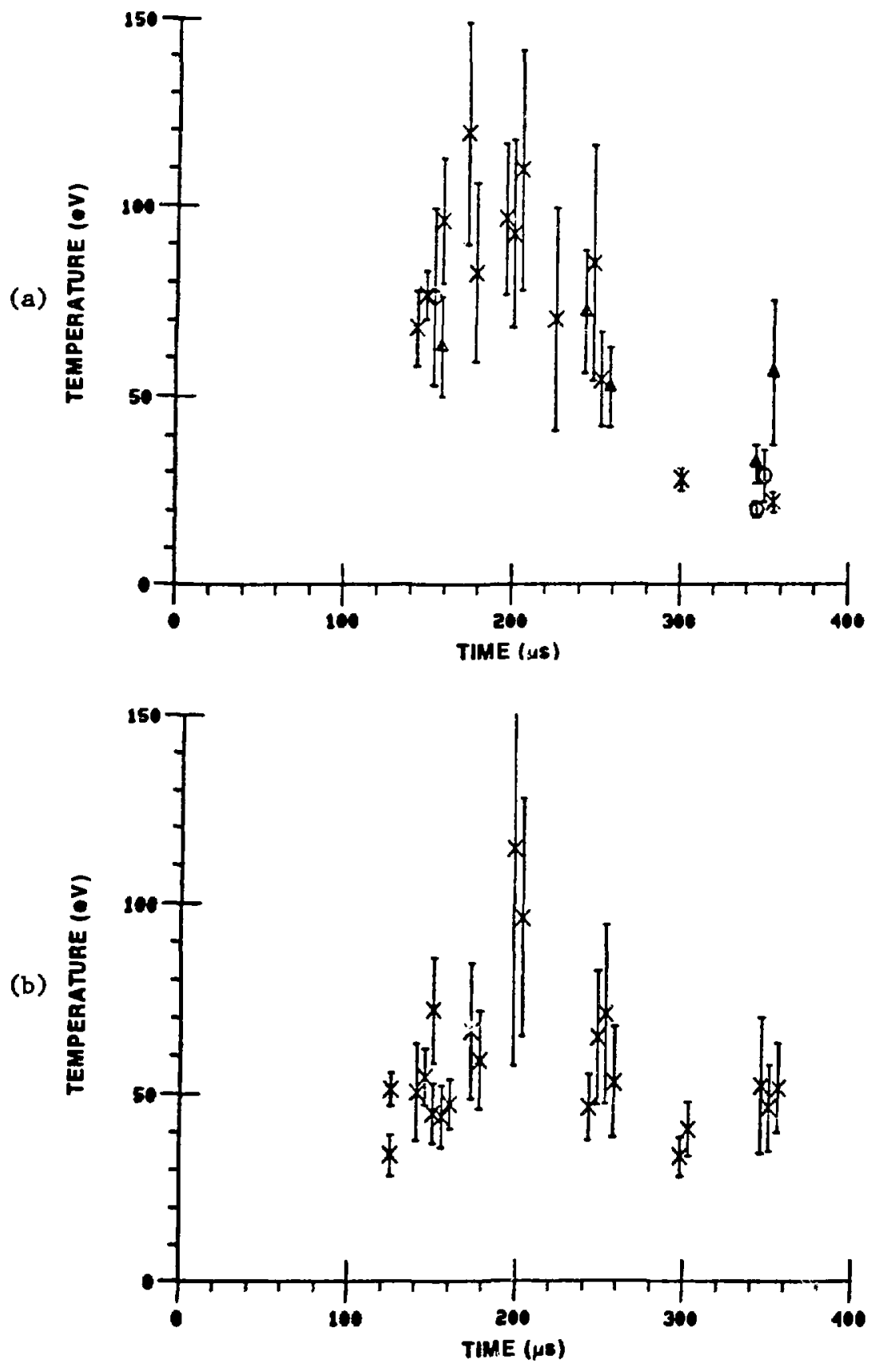

F1g. XI-15.

Atded reversal. Electron temperatures as functions of time: (a) $480 \mathrm{kA}$ and (b) $280 \mathrm{kA}$ peak toroldal current. F111 pressure 7.5 mtorr. The crosses, circles, and triangles in (a) are measured during different runs. The toroldal current peaks at $150 \mu \mathrm{s}$. 

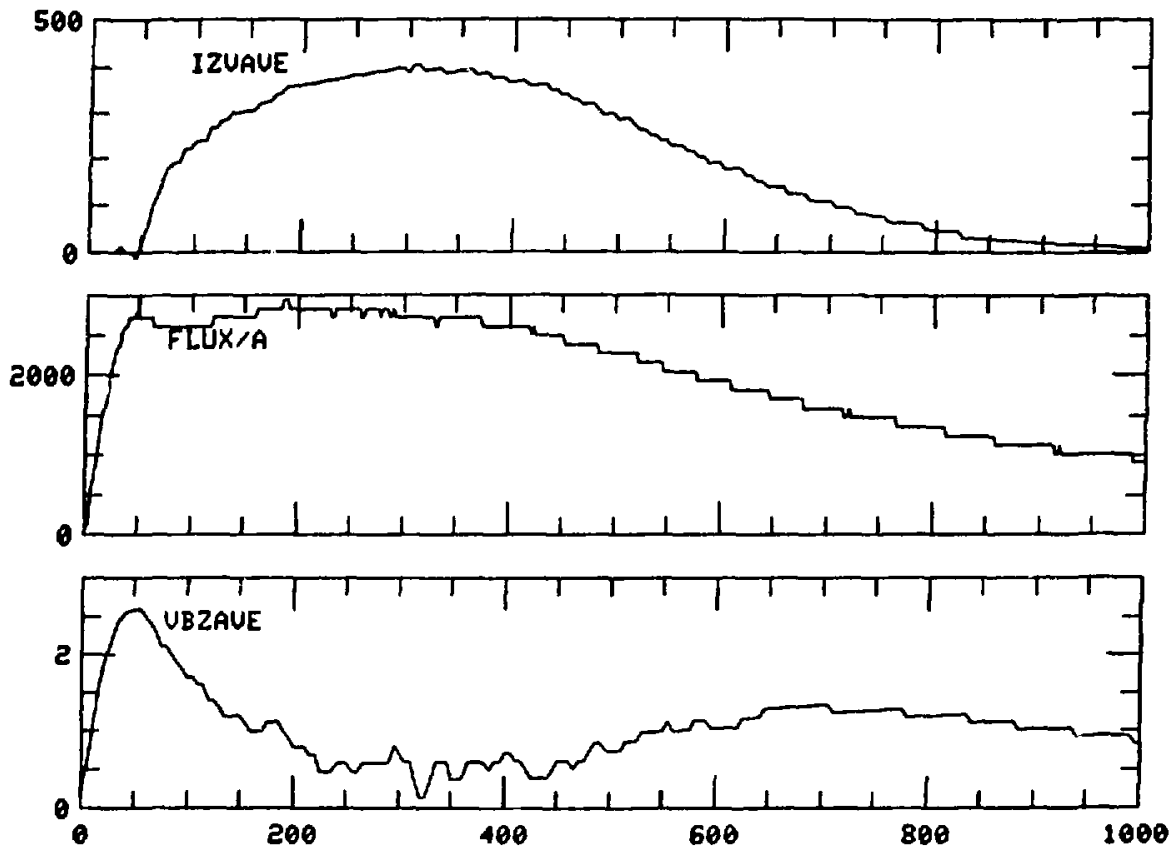

F1g. XI-16.

Single-feed configuration, stabilized pinch. Same display as in

F1g. XI-8.

light-gathering optic was Improved to make the spectrometer calibration less sensitive to the exact location of the scattering volume. These changes had no significant effect on the outcome of the measurements.

The situation will be explored further in future measurements. The fiber optics is being replaced by an array of forty-three 20-A-wide channels. These channels will be combined on a lesser number of photomultiplfers to look at the blue and red side of the spectrum over a wider range of temperatures. Also, If the effect is due to the plasma, it will be possible to explore the scattered spectrum with a higher resolution.

other improvements planned for the future Involve the computerized data acquisition. The dashed 1 ines in F1g. XI-5 w111 be implemented to determine the level of background radiation by interpolation rather than assumption of constancy. This will extend the useful range of the data acquisition systen to lower f111 pressures. 


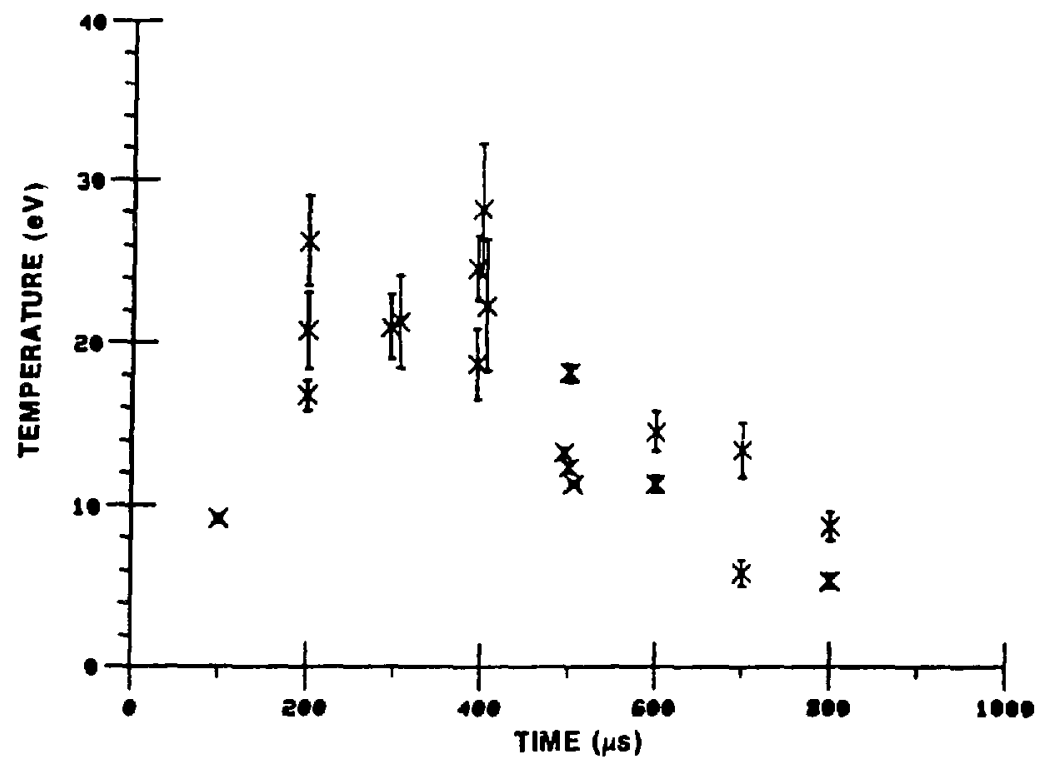

Fig. XI-17.

Single-feed configuration, stablilzed pinch. Electron temperature as function of time. F111 pressure--20 mtorr. Peak toroidal current- $400 \mathrm{kA}$ at $300 \mathrm{\mu s}$.

\section{ACKNOWLEDGMENTS}

The authors acknowledge helpful discussions with R. R. Bartsch, P. R. Forman, R. E. Siemon, and F. C. Jahoda. L. J. Jolin played an essential role in the setting up and testing of the diagnostlc. T. L. Petersen designed the photomultiplier gating circuit. K. S. Thomas was responsible for the operation of $\mathrm{ZT}-40$.

\section{REFERENCES}

1. M. Greenwald and W. I. B. Smith, App1. Opt. 16, 587 (1977)

2. K. B. Freese, R. R. Bartsch, and R. E. Siemon, "A Portable Thomson Scattering Apparatus for Use on the Scylla IV-P Theta Pinch," Bull. Am. Phys. Soc. 23, 848 (1978)

3. J. B. Taylor, Phys. Rev. Lett. 33, 1139 (1974) 
BASIC FEATURES OF ELECTRON DENSITY BEHAVIOR

by

A. R. Jacobson

The path-averaged electron density is measured along seven vertical chords simultaneously (see F1g. XII-1) using a $\lambda=3.39 \mu \mathrm{m}$ interferometer. 1 This chapter presents some very general observations relating to electron density profile, inventory, and fluctuations.

The interferometer measures chord integrals of $\mathrm{N}_{e}$ at seven locations in major radius, as shown in Fig. XII-1. If the density profile were peaked on axis, one would expect the average density along a chord to decrease with larger $\left|R-R_{0}\right|$. In fact, such peaking is rarely observed. The profile becomes leveled, or even hollow, usually before peak current is reached. Thereafter, the inward migration (due to $\vec{E} \times \vec{B}$ drifts in the ohmic heating electric field) is apparent1y weaker than the outward migration (which may be due to particle diffusion enhanced by fluctuations). For example, the data of F1g. XII-2 are the chord-averaged $\mathrm{N}_{e}$ as functions of offset major radius $\left(R-R_{0}\right)$ for 30 identical, 20-mtorr stabilized-pinch discharges. The data points are averaged over shots and over a 100- $\mu$ s period centered around peak toroldal current $(450 \mathrm{kA})$. The vibration-induced absolute uncertainty in $\mathrm{N}_{e}$ (averaged along a chord) 18 approximately $0.5 \times 10^{14} \mathrm{~cm}^{-3}$, Therefore the profile In Fig. XII-2 is consistent with a density distribution that is flat vs offset major radius, $R-R_{0}$. (This would transform to a flat distribution in minor radius as well, subject to a large uncertainty near $r=0_{0}$ )

Similar data are shown in Fig. XII-3 for a set of 14 self-reversing, 5-mtorr discharges. (The spatial point at $R-R_{0}=10.7 \mathrm{~cm} 1 \mathrm{~s} \mathrm{missing}$ due to poor alignment during the day on which the data were taken.) The density distribution may be hollow, even after allowing for vibrations 


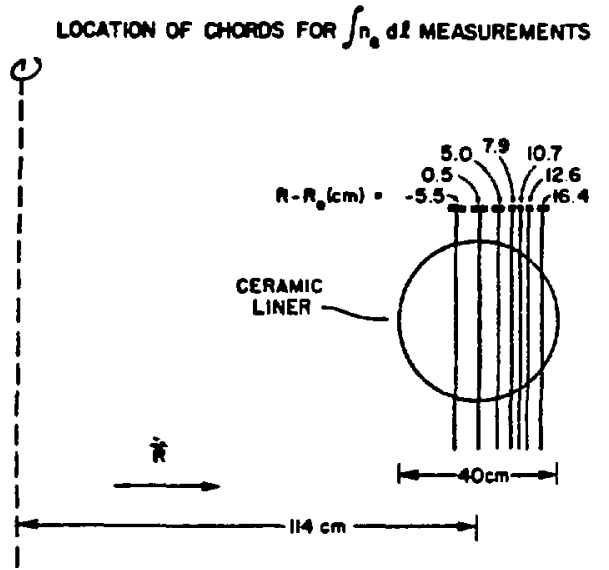

Fig. XII-I.

Arrangement of $\lambda=3.39 \mu \mathrm{m}$ interferometer chords on ZT-40.

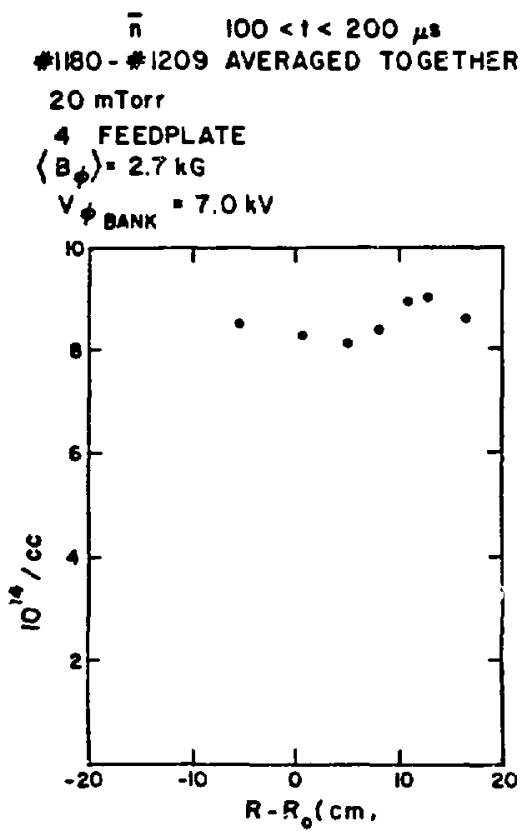

Fig. XII-2.

Chord-averaged electron density profile (in offset major radius, $R-R_{0}$ ) during peak current, averaged over 30 identical stabilized pinch discharges at 20 mtorr.

$\left(0.5 \times 10^{14} \mathrm{~cm}^{-3}\right)$. The data are averaged over shots and over a 100- $\mu$ s period centered around peak toroidal current ( $450 \mathrm{kA}$ ).

Finally, data for a series of low-current ( $\left.I_{\phi}=250 \mathrm{kA}\right), 20$-mtorr discharges are shown in FIg. XII-4. The 150-us averaging time is centered around peak current. The density profile is somewhat hollow in $R-R_{0}$, and this effect would be more pronounced in terms of $\mathrm{N}_{e}$ vs minor radius.

To summarize the electron density profile data, peaked distributions are rarely observed on $\mathrm{ZT}-40$, in contrast to the situation that occurs $1 \mathrm{n}$ the first several microseconds of a fast pinch. Instead, the profiles tend to be flat, or even hollow, vs offset major radius. (At 5 mtorr or below in ffll pressure, the effects of mechanical vibrations interfere with profile determination after approximately 200 us of current pulse.)

The electron inventiry (also known as "line denst:y," or electrons per unit axial length of the pinch) is re:dily measured by the multichord interferometer. Again, for times greater than $200 \mu s$ into the discharge (at fill pressures of 5 mtorr or less) the profile determination, and that of electron inventory, are unreliable because of mechanical vibrations. In the 


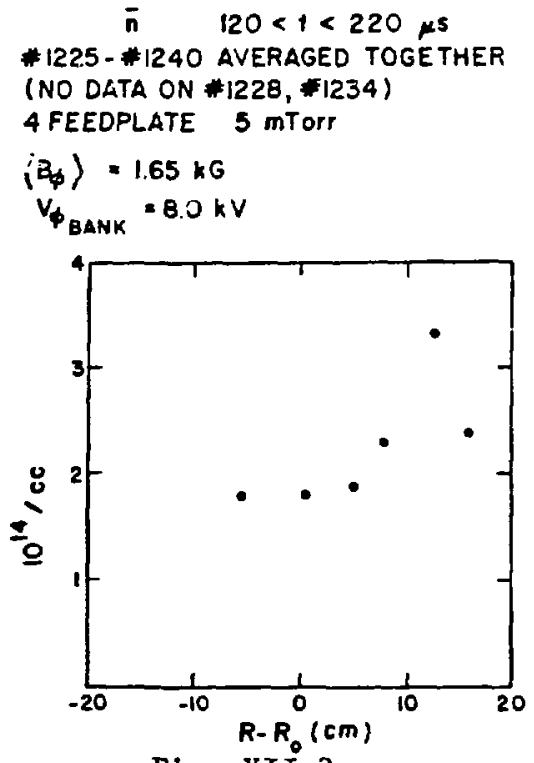

Fig. XII-3.

Chord-averaged electron density profile ( 1 n offset major radius, $r-R_{0}$ ) during peak current, averaged over 14 identical self-reversing discharges at 5 mtorr.
$\bar{H} \quad i 00<1<250 \mu \mathrm{s}$ 24 SHOTS AVERAGED TOGETHEH BE IWEEN \#I8IO AHD \#1843 20 mTOrP 4 FEEDPLATE

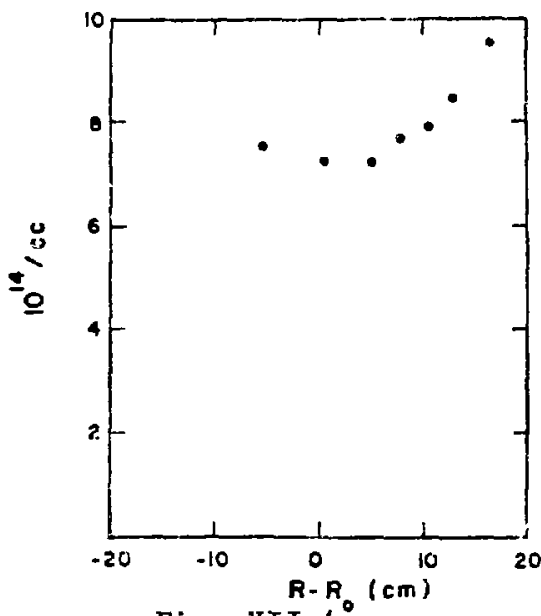

Fig. XII-4:

Chord-averaged electron densicy profile (in offset major radius, $R-R_{0}$ ) during peak current, averaged over 24 identical low-current, stabilized pinch discharges at 20 mtorr.

observations to follow, therefore, we will not deal with time suales longer than $200 \mu s$ or fill pressures lower than 5 mtorr.

The electron Inventory shows, to varying degrees, a "pump-out" effect, so that typically the plasma density (integrated over the pinch crcss section) is significantly less than the fully lonized gas fill. To begin with, the inventory increases monotonically during the first few tens of microseconds to $50 \%$ or more of the gas f111. Thereafter, in varying degrees, the inventory declines (over time scales as short as 20 or $30 \mu \mathrm{s}$ ) to some fraction of the attained peak. These two processes often occur by the time of peak curreni (approximately $100 \mu$ into the discharge), that is, during a period of strongly inward Poynting flux. Thus, the observed ejection of plasma during setup nften occurs in the presnnce of (and apparently in spite of) a strong inward $\vec{E} \times \vec{B} \mathrm{drift}$. The most striking feature of the electron inventory pumpout is that it becomes more extreme at lower fill pressure (all other things being held constant). This effect is seen in a wide varlety of discharge modes (stabilized pinch, self-reversal, aided reversal, and pitch programming). Data are shown in Fig. YII-5 for a series of 350-kA stabilized 
pinch discharges. The current starts at $t=50 \mu \mathrm{s}$ and peaks at $t=140 \mu \mathrm{s}$. Maximum inventory is reached by $t=90 \mu \mathrm{s}$. The data in Fig. XII-5 are the time-averaged inventory during the latter $40 \mu \mathrm{s}$ of the current rise. Each datum is one discharge. (The time scale is short enough that mechanical vibrations may be Ignored, even at the low pressure end.) Clearly the fractional inventory declines as the fill pressure is lowered; at $p=5$ mtorr, the time averaged inventory is only about $20 \%$ of the gas fill. This trend (toward lower fractional inventory as the fill pressure is decreased) is quite general and is not peculiar to stabilized pinch operation.

The final set of observations deals with electron density fluctuations. Since the correlation times of observed fluctuations are on the order of a few microseconds, the slow (hundreds of microseconds) phase shifts due to mechanical vibrations do not affect the fluctuation measurements.

The density fluctuations tend to increase as the fill pressure is reduced (all other things being held constant)。As in the case of pump-out (see above), this trend is apparent in any of the operating modes. (An important exception is provided by high-current, low-density RFP discharges in which a dramatic reduction in the density fluctuations is observed; such "density quiet periods" are discussed elsewhere in this report.) The data of Fig. XII-6 illustrate this trend. The discharges are the same as in Fig. XII-5 and each datum is one discharge. The averaging time is the latter $70 \mu s$ of the 90- $4 \mathrm{~s}$ current rise. Each datum is computed as follows: At every sampling time $t$ during the 70-us interval, for each chord a running average is calculated of the phase shift between $t-10 \mu \mathrm{s}$ and $t+10 \mu \mathrm{s}$. This running average is subtracted from the instantaneous phase at time $t$. The difference is then divided by the running average. In this manner a $\delta \mathrm{n} / \mathrm{n}$ is obtained (for each chord) as a function of time. The rms $\delta n / n$ (on each chord) is then calculated; finally, all seven chordal rms values are averaged. The data of Fig. XII-6 clearly illustrate the trend toward increased fractional fluctuations at lower $f 111$ pressures. It is noteworthy that for the same discharges, both the inventory pump-out (Fig. XII-5) and the fractional fluctuations (Fig. XII-6) scale similarly with pressure.

Another general feature of the density fluctuations is that outer chords show more (chord-averaged) turbulence than do central chords. This applies to a wide varlety of operating conditions, as will be 1llustrated below. The data are analyzed as follows: Along each measurement chord (see Fig. XII-1), 


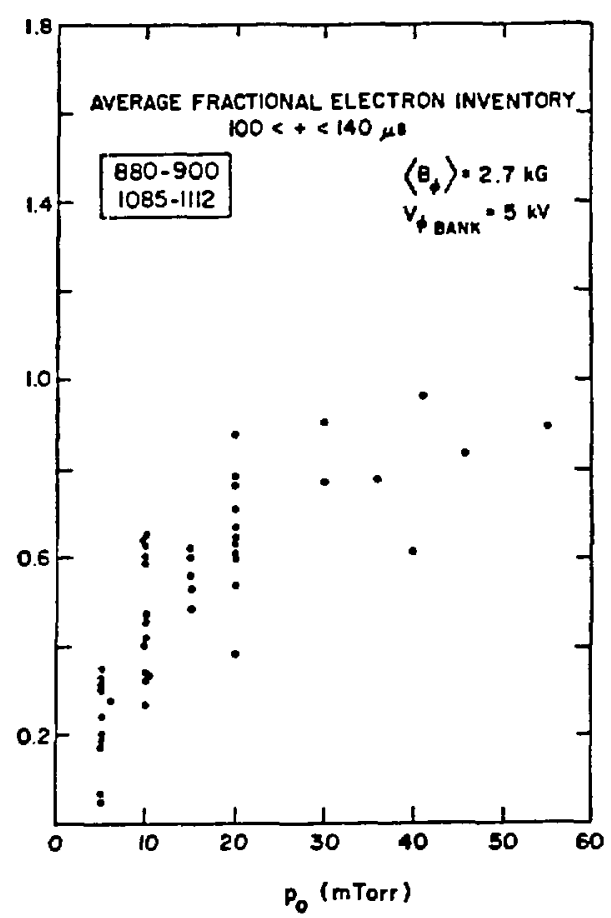

Fig. XII-5.

Electrons present (as a fraction of $100 \%$ lontzed fill) vs fill pressure for a series of stabllized pinch discharges. Each datum is one discharge. The averaging time is 40 Hs near the peak current.

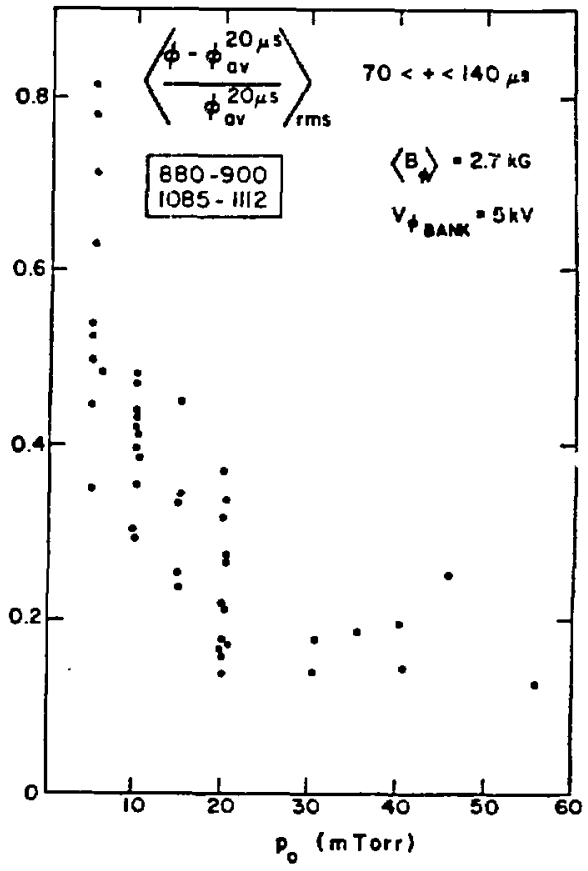

Fig. XII-6.

Fractional phase fluctuations vs f111 pressure for the same group of discharges as in Fig. XII-5. The averaging time is 70 us and includes part of the current rise and flattop.

the chord-averaged electron density $\bar{n}$ is tabulated as a function of time during an interval centered around peak current. The time derivative is then taken, and the rms of that derivative (for each chord) is calculated for the interval. This rms $\mathrm{d} \bar{n} / \mathrm{dt}$ is then plotted as a function of offset major radius. (It is not known at this time whether the turbulence is isotropic; thus, Abel inversion of the data, to obtain fluctuation level vs minor radius, is not justified.)

Data for a series of 30 identical 20-mtorr stabilized pinch ( $\left.I_{\phi}=450 \mathrm{kA}\right)$ discharges are shown in Fig. XII-7. For any particular discharge, the distribution of rms $d \bar{n} / d t$ varies widely from the series average. What is shown, then, is the statistically meaningful distribution after shot-to-shot irreproducibilities have been averaged away. This statistically significant 
distribution shows nearly twice as much fluctuation near the edge as near the center. The average density (not fluctuation) profile for the same series of discharges is shown in FIg. XII-1 and is essentially flat. This provides some evidence that $\delta \mathrm{n} / \mathrm{n}$ is larger near the edge than near the center. Figure XII-8 shows the statistical fluctuation profile for a similar series of shots, except at higher fill pressure (40 mtorr). Once again, the fluctuations are evidently edge-peaked.

In order to acquire data on density fluctuations under conditions of longer current risetime, the same statistical analysis has been applied to single-feedplate discharges. The averaging time (centered around peak

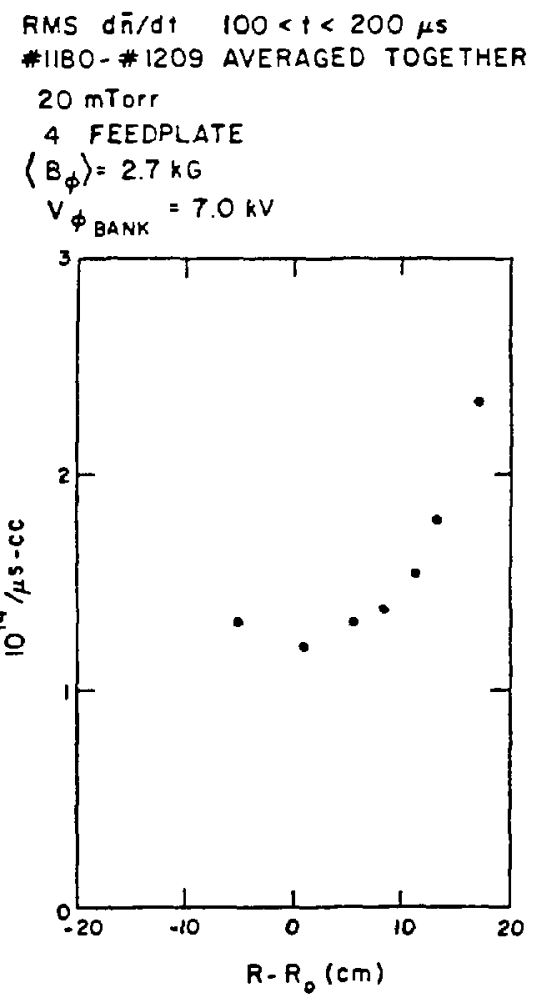

Fig. XII-7.

Chord-averaged density fluctuation profile (in offset major radius, $R-R_{0}$ ) during peak current, averaged over 30 identical stabilized pinch discharges at 20 mtorr.

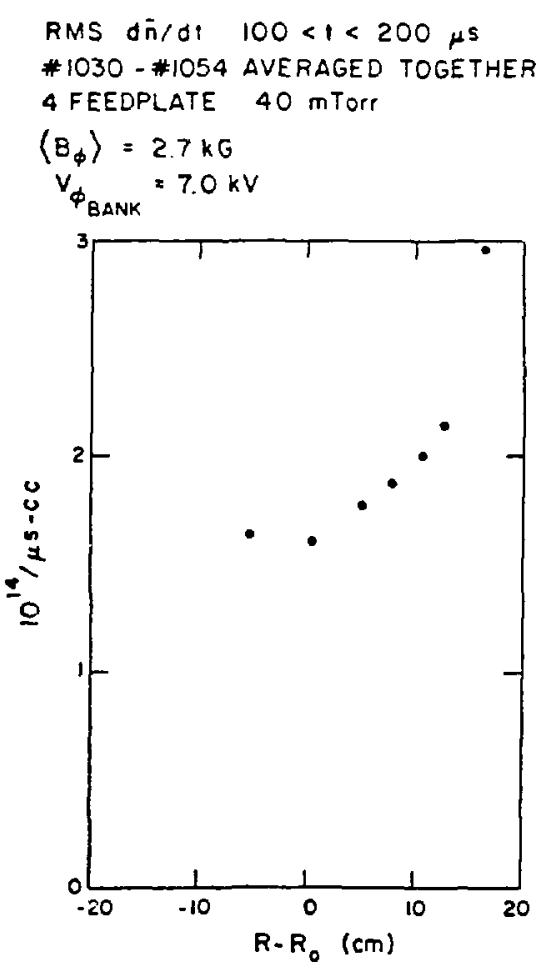

Fig. XII-8. Chord-averaged density fluctuation profile (in offset major radius, $R-R_{O}$ ) during peak current, averaged over 25 identical stabilized pinch discharges at 40 mtorr. 
current) is doubled (to $200 \mathrm{hs}$ ) because of the slower current waveform. Figure XII-9 shows the fluctuation profile for 21 identical 20-mtorr stabilized pinch discharges with $I_{\phi}=400 \mathrm{kA}$. Figure XII-10 shows data for a similar series of 18 discharges, except at 10 mtorr. In either case there is at least twice as much chord-averaged density fluctuation at the edge as through the center. Thus the edge-peaking of the density fluctuations is seen during peak current with either risetime attempted to date and is not peculiar to four-feedplate operation.
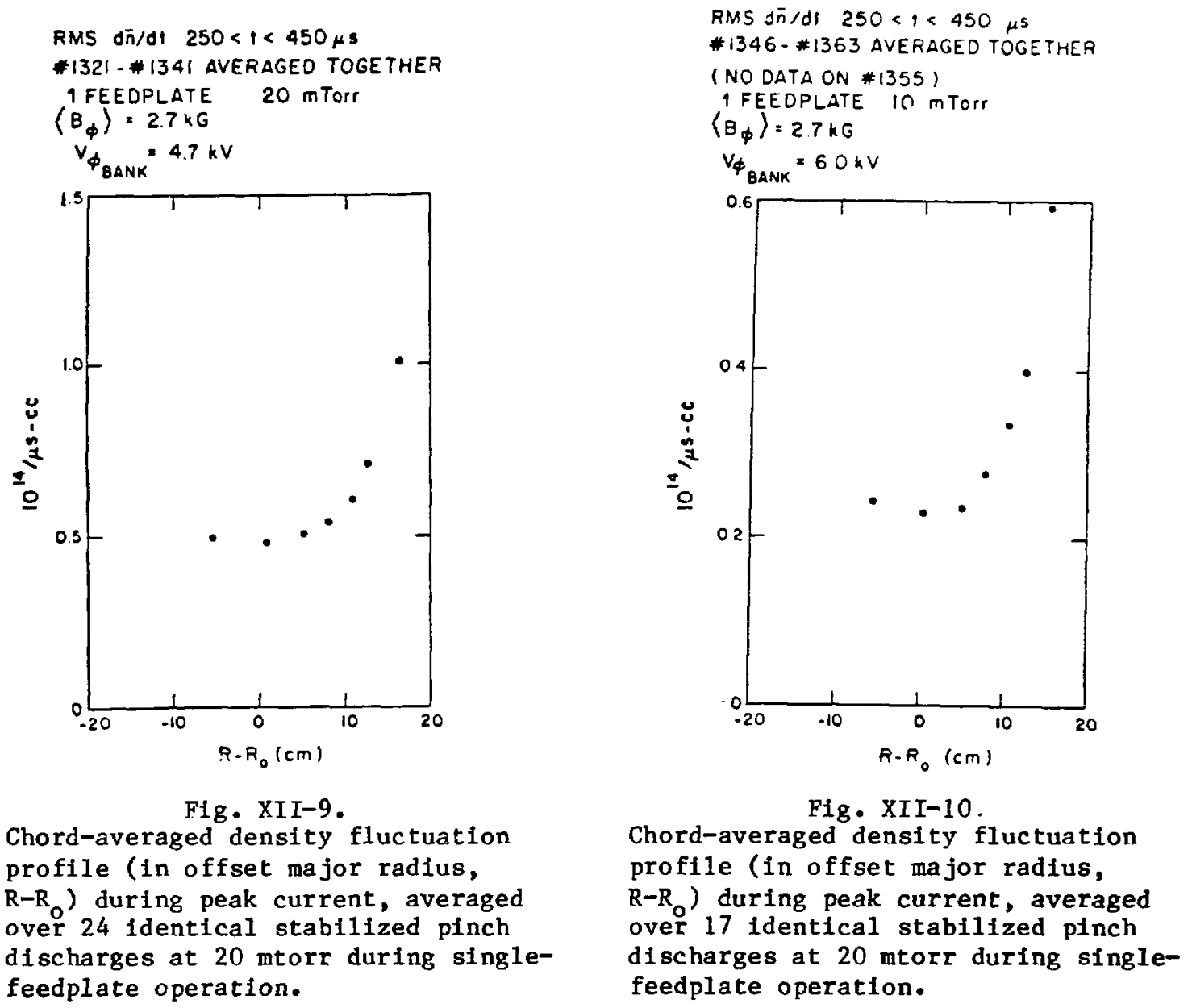
Self-reversal discharges with peak $I_{\phi}=500 \mathrm{kA}$ and 5 mtorr frequently show a dramatic reduction of the density fluctuations (see elsewhere in this report) during the decay of the current. However, during peak current these discharges are turbulent. Average turbulence profiles (during 100 us centered around peak current) are shown in FIg. XII-11, for 14 identical shots of this type, prior to the reduction of fluctuation level. The edge those are about is three times as high as those averaged along the central chord. The data of Fig. XII-3 (for the same 14 discharges) show less hollowness in the actual density profile. Thus, there is some evidence for peaking of $\delta \mathrm{n} / \mathrm{n}$ near the edge in the self-reversing type of discharge.
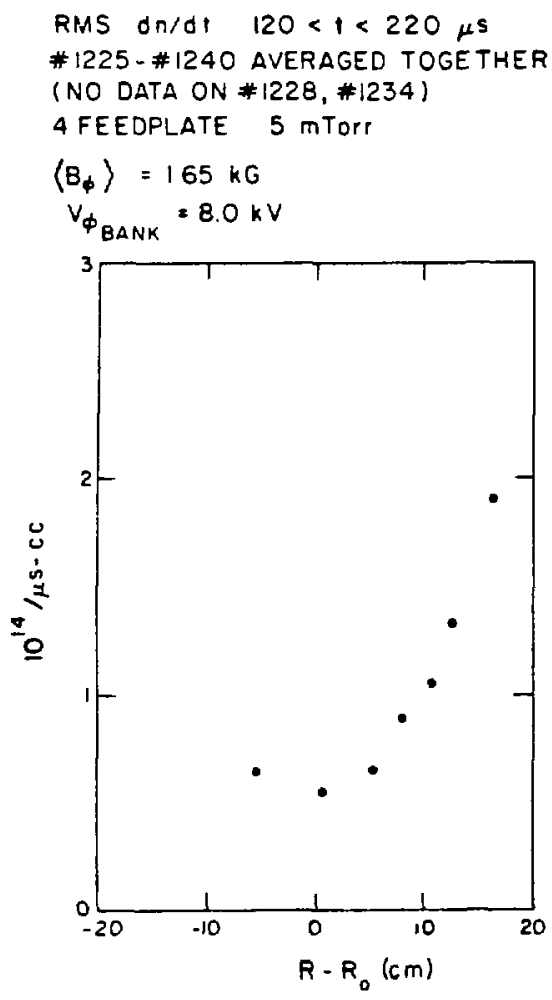

Fig. XII-11.

Chord-averaged density fluctuation profile (in offset major radius, $\mathrm{R}-\mathrm{R}_{\mathrm{o}}$ ) during peak current, averaged over 14 identical self-reversing discharges at 5 mtorr.

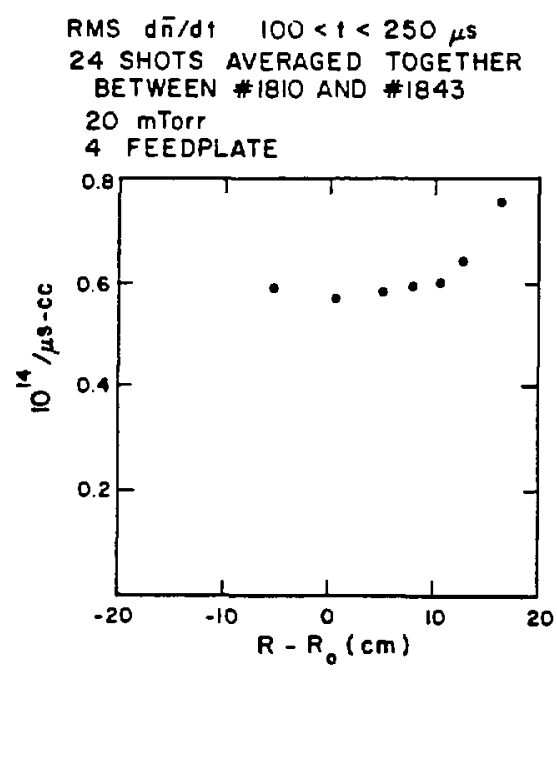

F1g. XII-12.

Chord-averaged density fluctuation profile ( $1 \mathrm{n}$ offset major radius, $\mathrm{R}-\mathrm{R}_{\mathrm{O}}$ ) during peak current, averaged over 25 identical low-current, stabilized pinch discharges at 20 mtorr. 
Finally, fluctuation distributions for a series of very low current $\left(I_{\phi}=250 \mathrm{kA}\right)$ discharges are shown in Fig. XII-12. Apparently the turbulence is less edge-peaked than at more standard current levels. The density profiles (see Fig. XII-4) are similarly hollow in $R-R_{0}$. Thus, at these low currents there is no evidence that $\delta n / n$ peaks at the edge.

The basic observations on plasma density behavior may be summarized as follows:

1. The density profiles are seldom peaked on axis and are more typically observed to be flat or even hollow in offset major radius $R-R_{0}$.

2. The electron inventory usually becomes lower than the fill, and this effect is more pronounced at lower fill pressures.

3. The fluctuations in density (averaged along chords) are genera11y peaked near the edge.

\section{REFERENCE}

1. A. R. Jacobson, "Heterodyne Quadrature Interferometer for Simultaneous Measurements of Plasma Density Along Several Chords," Rev. Sc1. Instrum. 49, No. 5, pp. 673-674, May 1978 . 
POLARIZATION OF MAGNETIC FLUCTUATIONS AT THE WALI

by

A, R. Jacobson and C. J. Buchenauer

Studies have been made of the fluctuating magnetic field at $r=r_{w}=21.8 \mathrm{~cm}$, which is close to the she11 $(r=22.0 \mathrm{~cm})$ and outside the ceramic liner. The probes, constructed by $\mathrm{C}$. J. Buchenauer, measure $\mathrm{B}_{\theta}$ and $\mathrm{B}_{\phi}$ at two poloidal locations separated by $\Delta \Theta=\pi$. One pair of colls is near the inside and the other near the outside, in terms of major radius. The signals are recorded as common-mode and differential-mode:

$$
\begin{aligned}
& \mathrm{B}_{\theta, \phi}^{(o)} \equiv \frac{1}{2}\left\{\mathrm{~B}_{\theta, \phi}^{\text {Inside }}+\mathrm{B}_{\theta, \phi}^{\text {outside }}\right\} \text { and } \\
& \mathrm{B}_{\theta, \phi}^{(1)} \equiv \frac{1}{2}\left\{\mathrm{~B}_{\theta, \phi}^{\text {Inside }}-\mathrm{B}_{\theta, \phi}^{\text {outside }}\right\}
\end{aligned}
$$

The common-mode field $\vec{B}(0)$ includes the equilibrium component and even-m perturbations (if any). The differential-mode field $\vec{B}(1)$ contains $|m|=1$ and $|\mathrm{m}|=3,5, \ldots$. perturbations (if any). It is convenient to reexpress the differential-mode fleld vector in terms of components parallel and perpendicular to the instantaneous common-mode field at the wa1l:

$$
\begin{aligned}
& B_{\|}(1) \equiv \vec{B}(1) \cdot \hat{e}_{\|}, \\
& B_{1}(1) \equiv \vec{B}(1) \cdot \hat{e}_{\perp} .
\end{aligned}
$$


These quantities as a function of time during a typlcal stabilized pinch discharge are shown in Fig. XIII-1. Clearly the magnetic disturbances are partially parallel and partially perpendicular. (Remember that the latter two axes are being constantly updated, since $B(0) / B(0)$ at the wall varies as a function of time.) Also apparent is a high degree of correlation between the two components.

We wish to study the "polarization" ratio $B\{(1) / B(1)$ as a function of discharge conditions. Because a portion of each component is uncorrelated with the other component, and because we wish to exclude the effects of slow offsets of elther signal (with respect to zero), it will be convenient to examine the ratio of two correlations:

$$
\frac{\left\langle\dot{\mathrm{B}}_{\|}^{(1)} \dot{\mathrm{B}}_{\perp}^{(1)}\right\rangle}{\left\langle\left[\dot{\mathrm{B}}_{\perp}^{(1)}\right]^{2}\right\rangle} \text {, }
$$
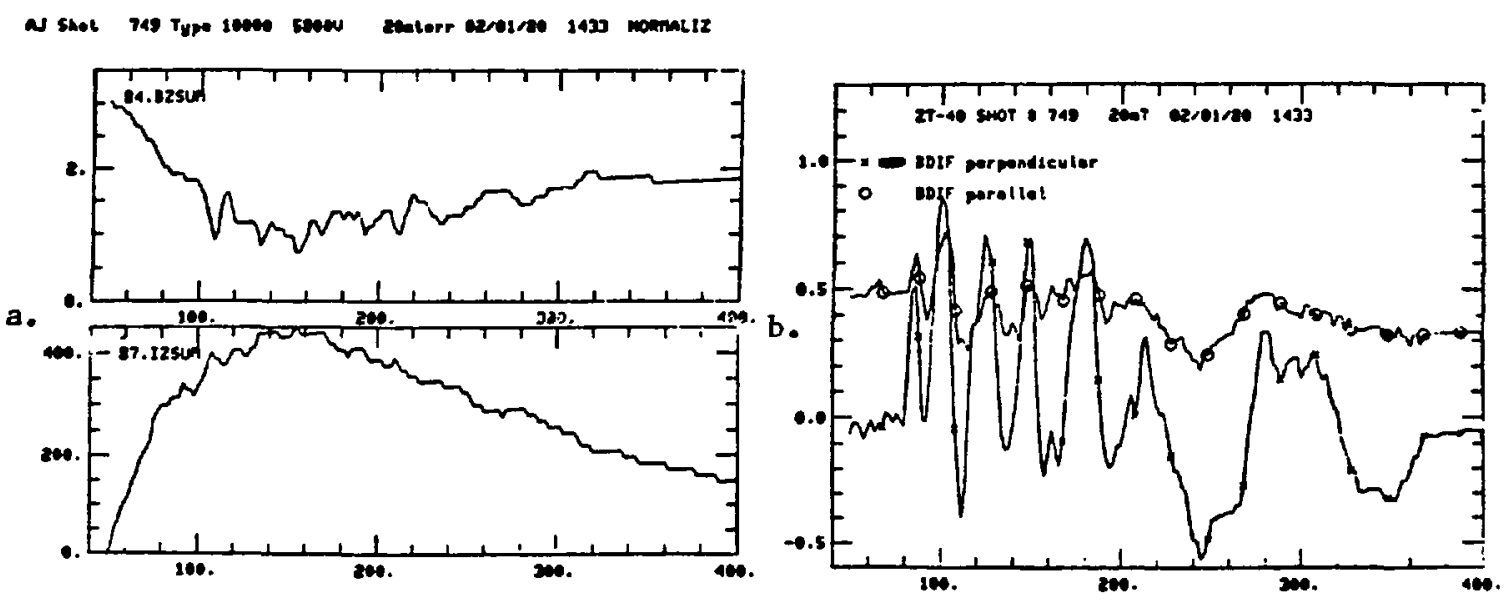

Fig. XIII-1.

(a) Toroidal magnetic fleld at wall (kG) and toroidal current (kA) vs time (us) for a nonreversing 20-mtorr discharge.

(b) Differential-mode magnetic flelds (kG) vs time ( $\mu s)$ for the same discharge. The curve marked by circles is the parallel component; the curve marked by $x^{\prime} s$ is minus the perpendicular component. 
where

$$
\begin{aligned}
& \left\langle B_{\|}^{(1)} \dot{B}_{\perp}^{(1)}\right\rangle=\int_{\tau} \dot{B}_{\|}^{(1)}(t) \dot{B}_{\perp}^{(1)}(t) d t \text { and } \\
& \left\langle\left[B_{\perp}^{(1)}\right]^{2}\right\rangle=\int_{\tau}\left[\dot{B}_{\perp}^{(1)}(t)\right]^{2} d t
\end{aligned}
$$

It should be remarked that the numerator is the "turbulence-weighted" polarization ratio, since

$$
\text { numerator }=\left\langle\dot{\mathrm{B}}_{\|}^{(1)} \dot{\mathrm{B}}_{\perp}^{(1)}\right\rangle=\left\langle\frac{\dot{\mathrm{B}}_{\|}^{(1)}}{\dot{\mathrm{B}}_{\perp}^{(1)}}\left[\dot{\mathrm{B}}_{\perp}^{(1)} \cdot 2\right\rangle\right.
$$

The averaging perlod, $\tau$, is chosen to colnctde with the flat maximum of $\theta$ versus $t$ in the discharge and is sufficiently short (50 $\mu$ s in the data to follow) that $F / \theta$ is reasonably constant throughout. It should be emphasized that, for the 1deal case of a single mode (in $\mathrm{m}, \mathrm{k}_{\phi}$, and $\omega$ ), the ratio of correlations reduces to $B(1) / B \overline{(1)}$ for that single mode.

A large varlety of 20-mtorr discharges (stabilized pinch, self-reversal, and alded reversal) with peak currents between $300 \mathrm{kA}$ and $500 \mathrm{kA}$ was studied. Plotted in Fig. XIII-2 is (minus) the ratio of the correlations for those discharges. These shots were accepted solely on the basis of two criterla: first, $P_{0}=20$ mtorr and second, the four transient digitizers Involved (al1 Biomation model $610 \mathrm{~B}^{\prime} \mathrm{s}$ ) recorded properly and did not saturate. For each data point (i.e., discharge), the position on the abscissa corresponds to $B(0) / B(0)$ averaged over $\tau=50 \mu \mathrm{s}$. The accual spread of each datum along the abscissa is $\Delta(F / \theta) \lessgtr 0.05$.

There are two striking features of the data shown in Fig. XIII-?. First, it has a preferred sign: $\dot{\mathrm{B}}_{\|}^{(1)}$ and $\dot{\mathrm{B}}_{\perp}^{(1)}$ are anti-correlated. Indeed, only one of the many shots examined contradicts this statement. Second, there is a trend toward larger values of the ordinate as the pinch becomes more deeply reversed. 
A plausible physical mechanism for the fact that $\dot{\mathrm{B}}_{\|}^{(1)}$ and $\dot{\mathrm{B}}_{\perp}^{(1)}$ are anticorrelated experimentally w111 be developed by considering a single mode (in $\mathrm{m}, \mathrm{k}_{\phi}$, and $\left.\omega\right)$. Since the probes cannot in fact resolve various $k_{\phi}$ or odd $\mathrm{m}$, the model offered here is inexact.

Fig. XIII-3 illustrates Imagined equilibrium field orientations at three minor radil for the case of an un-reversed pinch. At $r=0, \vec{B}(0)$ is purely toroidal (along $\hat{\phi}$ ). As $r$ increases, the fleld vector rotates progressively toward the pololdal direction. The field orlentation at $r_{w}$ (defined as the measurement position) is taken as the basis for the parallel-perpendicular coordinate system (differing from $\theta-\phi$ by a right-hand rotation about $\hat{\mathbf{r}}$ ), which is shown in Fig. XIII-4.

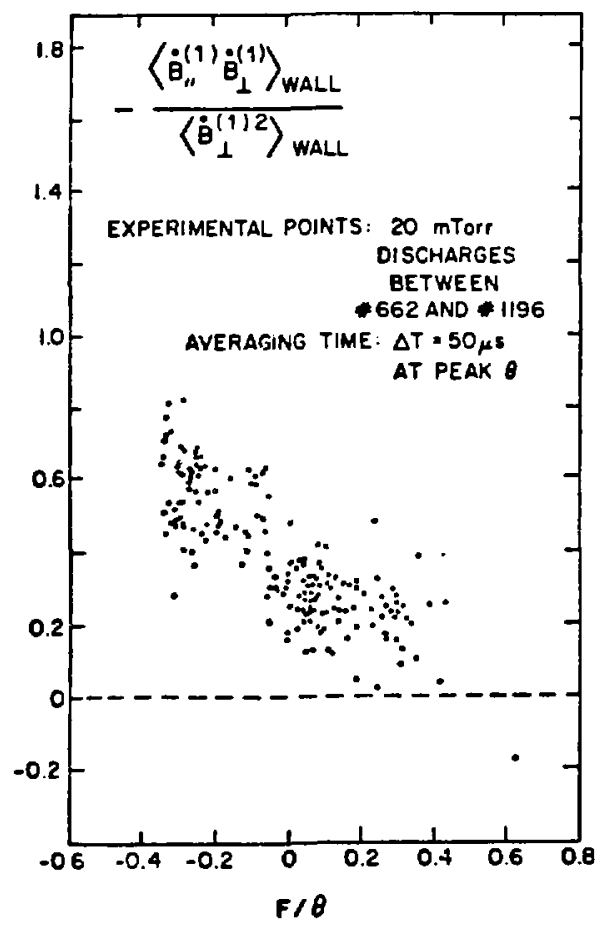

Fig. XIII-2.

Polarization of magnetic fluctuations as a function of $F / \theta=B_{\phi} / B_{\theta}$ at the wal1. Each datum is one discharge, averaged during a 50- $\mu$ s interval at peak $\theta$. All but one of the data points exhibits anticorrelation of $B_{\perp}^{(1)}$ and $B_{\|}^{(1)}$.

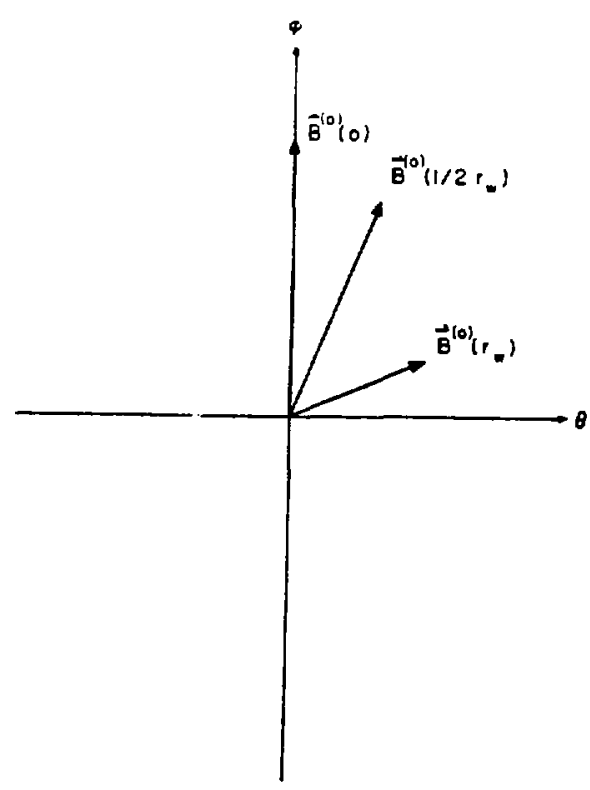

Fig. XITI-3.

Equilibrium magnetic fleld at three different minor radii, showing orientation in toroidal-puloidal "plane." 
The differential-mode magnetic: field

$$
\vec{B}(1)=B(1) \hat{e}_{\|}+B(1) \hat{e}_{\perp}
$$

could in principle lie in any quadrant within the coordinate system of Fig. XIII-4, and the corresponding polarization ratios are shown. (The experimental data 1ie in the second and fourth quadrants, chiracterized by a negative ratio.)

The differential-mode magnetic field at $r_{\mathrm{W}}$ is in a current-free region and is therefore expressible in terms of a scalar potential:

$$
\vec{B}^{(1)}=-\vec{\nabla}_{\phi}(1)=-\vec{k}_{\phi}(1),
$$

where

$$
\vec{k}=k_{\phi} \hat{\phi}+\frac{m}{r} \hat{\theta}
$$

Hence the experimental data of Fig. XIII-2 imply $\vec{k}\left(r_{w}\right)$ lying in the second and fourth quadrants of Fig. XIII-4 just like $\vec{B}(1)$, since $\vec{k}$ and $\vec{B}(1)$ are parallel (or antiparalle1). This situation is shown schematically in Fig. XIII-5, where the perturbation field at $r_{w}$ lies in the second quadrant, while the wavevector at $r_{w}$ lies in the fourth quadrant (relative to coordinate system of Fig. XIII-4). As one approaches $r=0$, the wavevector, $k(r)$, rotates toward the $\theta$-axis due to the fact that $k_{\theta}=m / r$. Referring to Fig. XIII-3, it is clear that at some intermediate radius, $0<r^{*}<r_{w}$, it is possible to satisfy the "resonant surface" condition:

$$
\vec{k}\left(r^{*}\right) \cdot \vec{B}(0)\left(r^{*}\right)=0
$$

Here, $r=r *$ is the location of perturbations whose pitch would match that of the equilibrium magnetic field. 


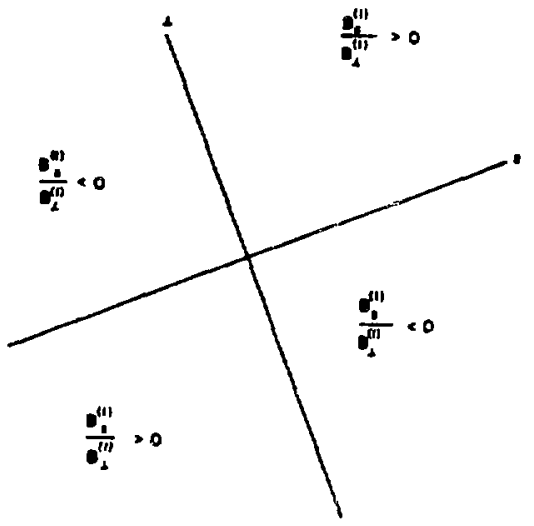

FIg. XIII-4.

The paralle1-perpendicular coordinete system (with respect to equilibrium magnetic field at wall) with the polarization ratios shown for each quadrant.

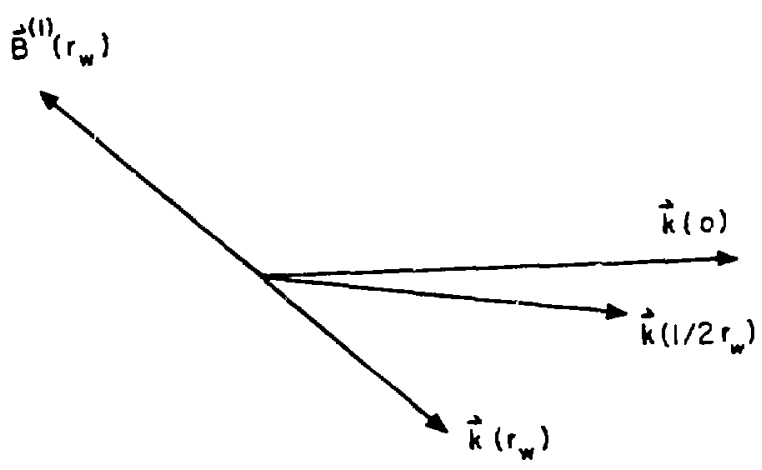

F1g. XIII-5.

Differential-mode magnetic field at vector (at wall) lying in second quadrant implies that associated wavevector (at wall) lies in fourth quadrant, with respect to parallel-perpendicular coordinate system. As the minor radius approaches zero, the wavevector rotates toward e direction.

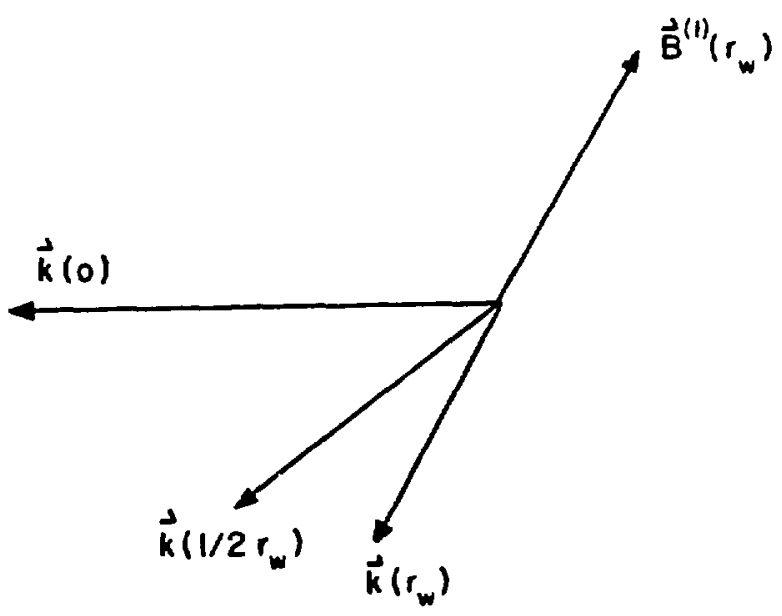

Fig. XIII-6.

Differential-mode magnetic field vector (at wall) lying in first quadrant Implies that assoclated wavevector (at wall) lies in third quadrant, with respect to parallel-perpendicular coordinate system. As the minor radius approaches zero, the wavevector rotates toward the minus $\theta$ direction. 
Consider the case of polarization opposite to what is found experimentally. This is shown in Fig. XIII-6, where $B^{(1)}\left(r_{w}\right)$ 1ies in the first quadrant (relative to the coordinate system of Fig. XIII-4), so that $B(1) / B(1)>0$. As in the other situation, $\vec{k}$ rota:ns in the $(-) \theta$ direction as one goes inward in minor radius. Comparison wis the equilibrium magnetic fleld behavior (FIg. XIII-3) shows that the "iesinant surface" criterion cannot be satisfied except exactly on axis, $r^{*}=0$.

Thus, the two cholces of polarization [sign of $B(1) / B(1)$ ] imply--within the 1dealized linear, single-mode picture-qualitatively different mechanisms for the origin of the measured fluctuations. It is noteworthy that experimentally we find $B(1) / B(1)<0$, which 1 s consistent with "resonant surfaces" at which

$$
\vec{k}\left(r^{*}\right) \cdot \vec{B}^{(0)}\left(r^{*}\right)=0
$$

Another approach to the polarization data is to assume that all the magnetic fluctuations observed at $r=r_{w}$ arise from interior "resonant surfaces" and then to infer some logical consequence regarding the radial profile of $\vec{B}^{(0)}$. Specifically, consider what would happen if a large "vacuum" region existed in the outer edge of the pinch, in which $B_{\phi}=$ constant and $B_{\theta} \sim 1 / r$. "Resonant surfaces" in that outer region would then generate polarizations such that $B(1) / B(1)>0$ at $r=r_{w}$, as can be seen by arguments similar to those of Figs. XIII-3-6. Since the data does not show such a polarization, the implication would be that such a "vacuum" region, if there is one in $7 \mathrm{~T}-40$, does not play a role here.

Arother model-dependent piece of information contained in the polarization data of Fig. XIII-2 is the actual location, in minor radius, of the hypothesized "resonant surfaces." The Idealized 11near, single-mode analysis will be used in deriving this further information; therefore, caution is advisable in comparing the (naive) theory with the (complicated) experimental results.

Consider the critical layer $r=r *$ where

$$
\vec{k}\left(r^{*}\right) \cdot \vec{b}(0)\left(r^{*}\right)=0
$$


or

$$
k_{\phi} B(0)\left(r^{*}\right)+\frac{m}{r^{*}} B_{\theta}^{(0)}\left(r^{*}\right)=0,
$$

which 1mplies

$$
\frac{\mathrm{B}_{\phi}^{(0)}\left(\mathrm{r}^{*}\right)}{\mathrm{B}_{\delta}^{(0)}\left(\mathrm{r}^{*}\right)}=\frac{-\mathrm{m}}{\mathrm{kr^{* }}} .
$$

At the location of external measurements,

$$
\vec{B}^{(1)}=-\vec{\nabla}_{\phi}(1),
$$

so that

$$
\begin{aligned}
\frac{B_{\phi}^{(1)}\left(r_{w}\right)}{B_{f}^{(1)}\left(r_{w}\right)} & =\frac{k r_{w}}{m} \\
& =\frac{-r_{w}}{r^{*}} \frac{B \delta^{(0)}\left(r^{*}\right)}{B_{\phi}^{(0)}\left(r^{*}\right)} .
\end{aligned}
$$

The parallel and perpendicular perturbation fields are, respectively,

$$
\begin{aligned}
& B_{\|}(1) \equiv \vec{B}(1) \cdot \hat{e}_{H}, \\
& B_{\perp}(1) \equiv \vec{B}^{(1)} \cdot \hat{e}_{\perp} .
\end{aligned}
$$

Combining these identities, it is possible to relate the polarization ratio to the equilibrium field:

$$
-\frac{B\}^{1)}\left(r_{w}\right)}{B\left\{^{(1)}\left(r_{w}\right)\right.}=\frac{-\frac{r_{w}}{r^{*}} \frac{B_{\theta}^{(0)}\left(r^{*}\right)}{B_{\phi}^{(0)}\left(r^{*}\right)} \frac{B_{\phi}^{(0)}\left(r_{w}\right)}{B_{\theta}^{(0)}\left(r_{w}\right)}+1}{\frac{B}{B(0)\left(r_{w}\right)}+\frac{r_{w}}{B_{\phi}^{(0)}\left(r_{w}\right)} \frac{B^{(0)}\left(r^{*}\right)}{B_{\phi}^{(0)}\left(r^{*}\right)}}
$$


This expression can be evaluated (in the Bessel function model) for various ratios $r * / r_{w}$ as a function of

$$
\frac{F}{\theta}=\frac{B_{\phi}^{(0)}\left(r_{w}\right)}{B_{\theta}(0)\left(r_{w}\right)}
$$

The curves are shown in Fig. XIII-7. Comparison with the experimental data (FIg. XIII-2) shows that the data 1 le between the Bessel function model curves for $r^{*}=r_{w}$ and $r^{*}=\frac{1}{2} x_{w}$. That is, if $\vec{B}^{(0)}$ follows Besse1-11ke profiles, and if (as the data seem to suggest) "resonant surface" phenomena are Involved, then the "resonant surfaces" would appear to lie in the outer half of the discharge tube, over a wide range of $F / \theta$.

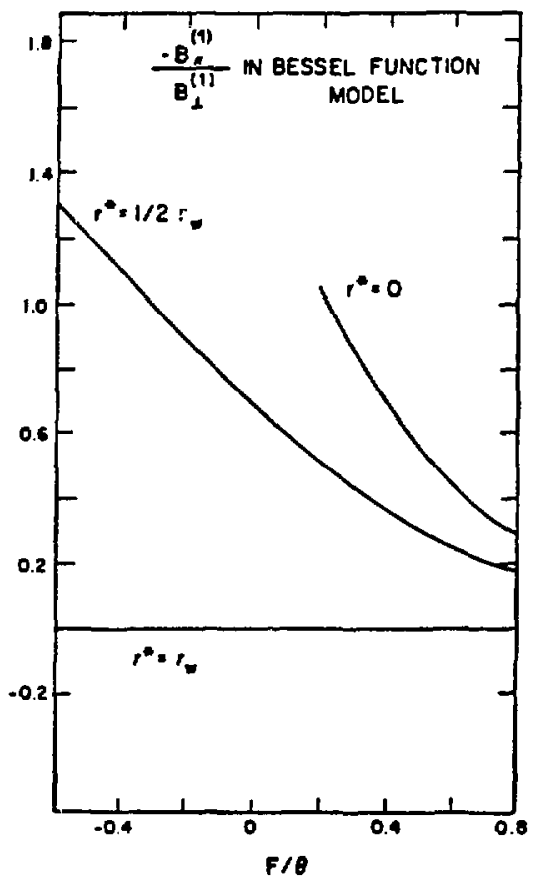

FIg. XIII-7.

Polarization ratio (as a function of $B_{\phi} / B_{\theta}$ at wa11) in the Besse1 function force-free model. 
CHAPTER XIV

POLOIDAL FLUX INPUT AS A FUNCTION OF TOROTDAL CURRENT RISETIME

by

J. A. Phi111ps

To obtain information on the resistivity of the $7 . T-40$ discharge, the pololdal flux input was measured by integration of the toroidal voltage $V_{e}$ across one feedplate. As $\int_{0}^{t} v_{z} d t=\phi_{\theta}(t)+\int_{0}^{t} v_{t}(0, t) d t$, where $\phi_{p}(t) \quad 1 s \quad t h e$ discharge poloidal flux (Including that between the inside of the porcelain tube and aluminum primary) and $v_{t}(o, t)$ is the voltage along the axis, the loss In flux $\int_{0}^{t} v_{z}(0, t) d t$ gives a measure of the discharge resistance.

ZT-40 has been run with four different risetimes, 33, 45, 95, and 230 , and Inittai gas pressures between 7 and 30 mtorr (FIgs. XIV-1 and XIV-2). The 95-us risetime was that for normal operation with four feedplates, with a charging voltage of $5.0 \mathrm{kV}$ and crowbarring at the peak current of $\sim 360 \mathrm{kA}$.

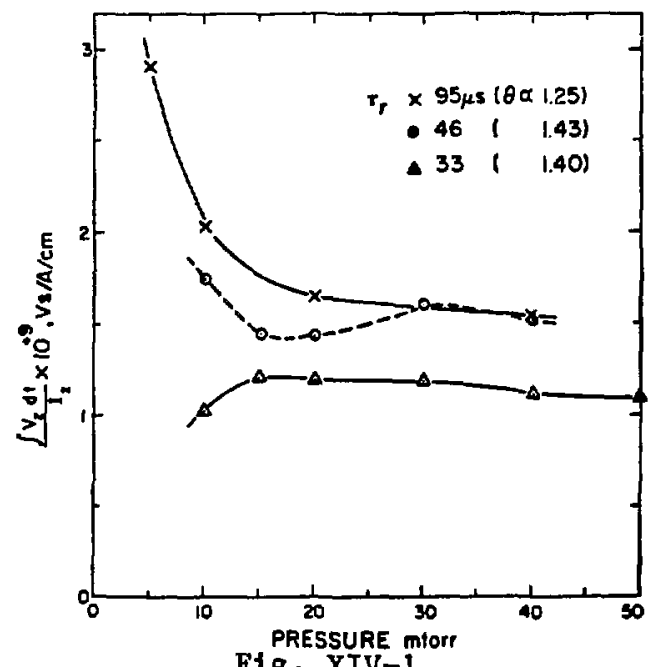

Fig. XIV-1.

Volt-second input at peak current vs pressure for three current risetimes.

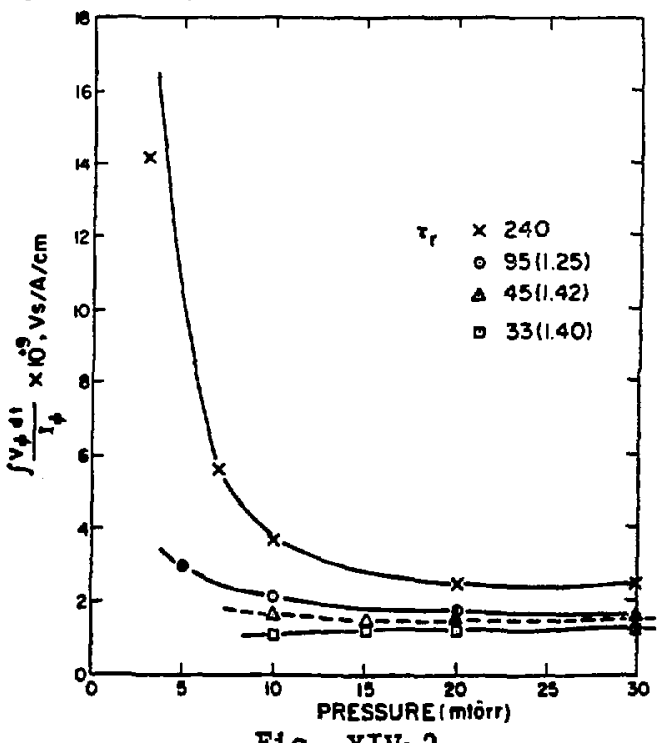

F18. XIV-2.

Vo1t-second input at peak current vs pressure for four current risetimes. 
The shorter risetimes were achieved by charging voltages of 7.5 and $10.0 \mathrm{kV}$ and crowbarring the discharge at a current level of $360 \mathrm{kA}$, or at $\sim 45^{\circ}$ and $30^{\circ}$, respectively. The torotdal field was held constant for these three risetimes with a peak $\theta$ of $\sim 1.4$. Single feedpoint operation gave a current risetime of $230 \mu \mathrm{s}$. Pressure runs with the 230-us risetime were made at a constant toroldal magnetic field but as the peak toroidal current dropped at

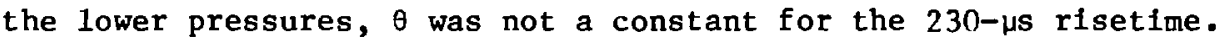

An examination of the results, Figs. XIV-1 and XIV-2, shows the following.

1. With the fastest risetime, $33 \mu \mathrm{s}$, the flux input is relatively constant with pressure and is somewhat less than that for a fully diffused current channel (uniform current density) of pinch radius, $r_{p}=14.5 \mathrm{~cm}$, calculated from pressure balance (plasma pressure zero). The loss of flux on the axis is therefore small.

2. With the longer risetimes, and at pressures $>20$ mtorr, more flux enters the discharge, with increased flux annihilation on axis. For a 240-us risetime the input flux is almost twice that for the shorter risetimes. This trend is expected since at the low plasma temperatures seen in these discharges, the resistance is relatively high, and the poloidal field has diffused to the axis in the first quarter-cycle. The longer this quarter-cycle the more the annihilation of flux on the axis. 3. As the pressure is reduced, the discharge is seen to become more resistive, drastically seen in the case of a 240- 4 s risetime, where the flux Input at 3 mtorr is $\sim 5$ times that at 30 mtorr. The high plasma resistance is reflected in the current which is highly damped at the lower pressures; e.g., at 5 mtorr the peak current is $\sim 3$ times smaller that at 30 mtorr. This behavior with pressure supports observations on ZT-40 that at low pressures there are large fluctuations in density, magnetic flelds, and discharge current, showing that there are strong anomalous resistivity effects.

\section{DISCUSSION}

From the data taken with magnetic probes reported in the following section, it was found that a maximum of abuut $33 \%$ of the Input flux was annihflated on the axis at peak current. The data was taken with the ZT-40 operated with a current risetime of $230 \mu \mathrm{s}$ and pressure of 20 mtorr. 
Using this result and assuming that the magnetic fleld profiles are similar for all four current risetimes reported here, the fractional loss of input flux for the four risetimes can be calculated with the result shown in Table I.

These results support the calculation of discharge inductance reported earlier from pressure balance. For all cases the data show that the poloidal field has probably diffused rather fully to the axis.

In the paper on pressure-balance limitations in $z$ pinches with diffusion heatingl it was shown that "with discharge currents which rise to the final value rapidly compared to the compression and flux diffusion times the pinch cannot achieve static equilibrium after the poloidal field diffuses to a uniform current distribution unless losses or plasma pressure at the wall are present." In the present experiments these conditions are not met since the current risetimes are relatively slow, and complete diffusion to a uniform current distribution, $B_{\theta}$ rising 1 inearly with radil, occurs at about current maximum. Tom 0liphant has, however, considered such cases with the numerical code, RAVEN, and has found $\mathrm{B}_{\theta} \geqslant 1$ early in the current rise. From these arguments it appears that, in all the current risetimes considered here, the discharge without losses would have been heated to a $\beta \geqslant 1$ by the time of current peak.

If it is assumed for the three fastest risetimes, where $I_{p} \simeq 370 \mathrm{kA}$ and initial pressures are 20 mtorr, that 1) 50\% (optimistic) of the initial gas fill remained in the discharge at current peak and 2) the plasma temperatures (optimistic) were $T_{1}=T_{e}=40 \mathrm{eV}$, the caleulated $\beta_{\theta}$ is $\sim 16 \%$. It thus appears that the discharge loses more than $84 \%$ of the energy transferred to it by diffusion heating. Absolute measurements of radiation and particle losses are necessary to support this conclusion.

TABLE I

FLUX INPUT AS A FUNCTION OF RISETIME

Current Risetime ( $\mu s)$
Fraction of Input Flux Annihilated on Axis ( $)$ 
Incidentally, as the absolute quantity of energy that is lost by the plasma is almost independent of risetime for the three fastest risetimes, it

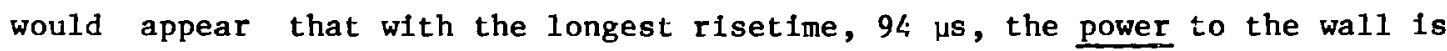
the smallest. The temperature rise and damage to the wall would then be the lowest for the longest risetimes.

REFERENCE

1. D. A. Baker and J. A. Phillips, Phys. Rev. Lett. 202, 32 (1974) 
CHAPTER XV

MEASUREMENTS OF MAGNETIC FIFLDS IN ZT-40

by

L. C. Burkhardt and J. A. Phillips

\section{INTRODUCTION}

Preliminary runs have been made with a magnetic probe in ZT-40 to determine the magnetic field profiles and to investigate the properties of toroidal current sheaths near the tube wall at early times in the discharge cycle. The existence of a relatively thin sheath, thickness a few centimeters, has been inferred from fast toroldal current rates of rise and from density interferometry measurements which suggest plasma accumulation near the tube wall.

\section{EXPERIMENTAL CONCERNS}

When the probe was run in the $\mathrm{ZT}-40$ discharge, there was a finite probability that the sapphire jacket would break and 1mpurities would be released from the probes (encased in epoxy), which would destroy the cleanliness of the vacuum chamber. It was therefore considered desirable to have in the first probe runs a probe assembly made out of those materials which exist in the discharge system, namely, ceramic and aluminum. The design and calibration of two probes is described below.

Two 1imitations of the first probe should be mentioned.

1. As there was only one coil pickup, several shots were required to map out the magnetic field profile; shot-to-shot reproducibility was, in general, good, $\pm 15 \%$.

2. As insulating materials, epoxy or plastic were not to be used; the aluminum twisted-pair connecting the coll to the outside was not ideal. As described below, theie was a significant contribution, up to $\sim 20 \%$, to the probe signal by the twisted-pair. 
In the experiment the magnetic probe was calibrated against the known peak toroidal field with the probe located at a minor radius of $6 \mathrm{~cm}$. Two discharges were made at each radius and the two probe signals averaged. The radial interval, $10-20 \mathrm{~cm}$, was covered during the run.

\section{DATA ANALYSIS}

Examination of the data with the first probe revealed the following behavior of the probe.

1. Perturbations in the toroidal field due to the hole in the primary for the diagnostic port were measured to be $15 \%$ larger than earlier results. The leads in the twisted-pair may be the cause of the discrepancy.

2. The discharge current calculated from the $B_{p}$ probe signal at $20 \mathrm{~cm}$ is $\sim 20 \%$ less than the known toroidal current. Either pickup from the twisted-pair or significant currents flowing outside the probe could be responsible.

The discharges examined with the first probe had the following parameters.

- Mode of operation: (1)(1) - stabilized, with $I_{\phi}$ crowbarred at $350 \mu \mathrm{s}$.

- One-feedplate operation: $\mathrm{I}_{\phi}$ peak $\pm 250 \mathrm{\mu s}$.

- Voltages: $\mathrm{v}_{\phi}=4.7 \mathrm{kV}, \mathrm{v}_{\theta}=8 \mathrm{kV}$.

- Peak $I_{\phi} \simeq 360 \mathrm{kA}$.

- Gas pressure: 20 mtorr.

- Discharges analyzed: 1585-1617.

The measured magnetic field distributions and calculated toroidal current densities are shown in Figs. XV-1 through XV-4.

A number of significant observations can be made.

1. At early times, $F i g \cdot X V-1$, the $B_{p}(r)$ profiles show a slow increase of field between $10-$ and $20-\mathrm{cm}$ radii with no strong evidence for a pronounced current sheath moving radially inward.

2. There is no strong evidence for a current concentration close to the wall even at late times after the toroidal current maximum. 
3. The poloidal magnetic field distributions are almost constant with radius and approximate that for $\mathrm{A} 1 / \mathrm{r}$ current density distribution. 4. The toroldal magnetic field behaves as in other experiments, peaking near the center of the discharge tube and falling off almost linearly with radius. In this run there was no evidence for field reversal, in agreement with measured values of $B_{\phi}(w)$.
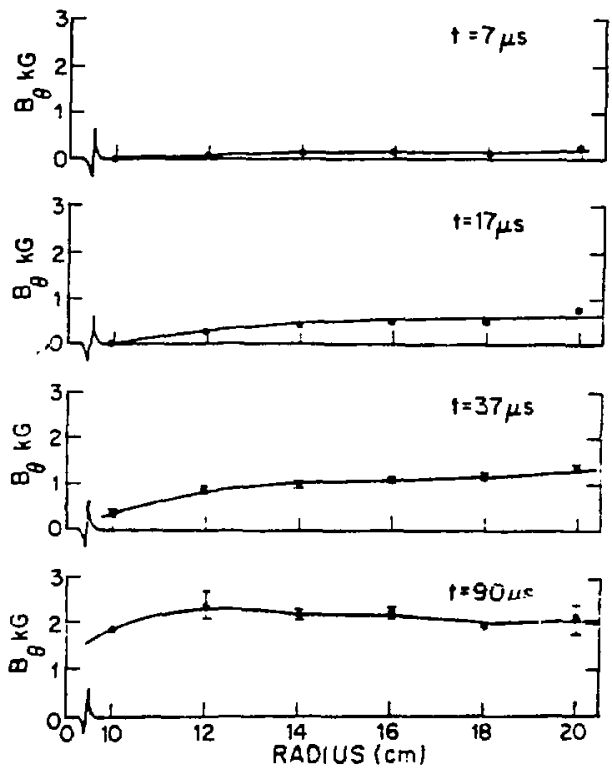

Fig. XV-1.

Magnetic probe data on the magnetic field component $B$ poloidal $\left(B_{0}\right)$ at early times ( 7 to 90 microseconds) in the discharge as a function of radius.
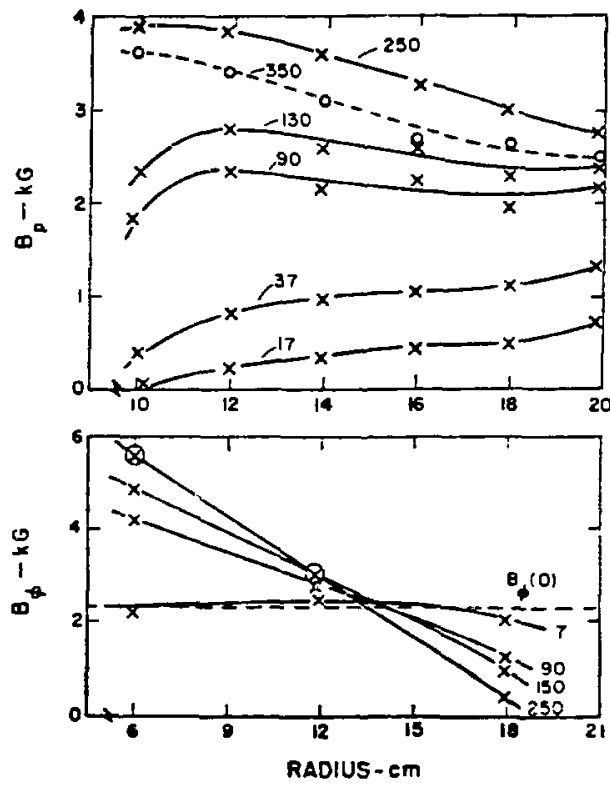

Figs. XV-2 and XV-3. Magnetic probe data plotted as a function of radius for $B$ poloidal and $B$ toroidal at times ranging from 0 to 250 microseconds in the discharge. 


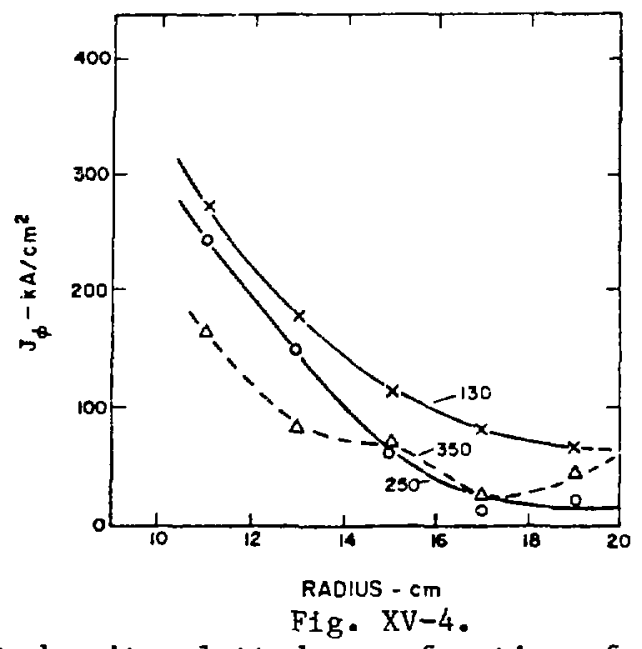

Toroidal current density plotted as a function of radius for times 130 , 250 , and $350 \mathrm{microseconds}$ in the discharge.

With the measured poloidal field distribution between 10 and $20 \mathrm{~cm}$, an inventory of the various components to the volt-second measured at the header can be made, as shown in Fig. XV-5. These components are, starting from the base line:

1. $\& \int_{10}^{20} \mathrm{~B}_{\mathrm{p}} \mathrm{dr}$ is the integrated poloidal flux between 10 and $20 \mathrm{~cm}$; $l$ is the major circumference of the torus.

2. The flux, or volt-second in the cables shorting out the three headers and in the space between the inside of the porcelain discharge tube and aluminum primary, is calculated by $L I_{t}$, where $L=165 \mathrm{nH}$. (The volt-second due to these inductances is added to that of $\& \int_{10}^{20} B_{p} d r$ in the figure.)

3. The volt-second due to the resistance in the cables $(780 \mu \Omega)$ is calculated from $R I(t)$ and time integrated.

4. The poloidal-field distribution inside of $\mathbf{r}=10 \mathrm{~cm}$ was estimated assuming a uniform current distribution inside $r=10 \mathrm{~cm}$.

5. The total $\int v_{z}(t) d t$ fed into the header is simply the integrated voltage at the one feedplate. 


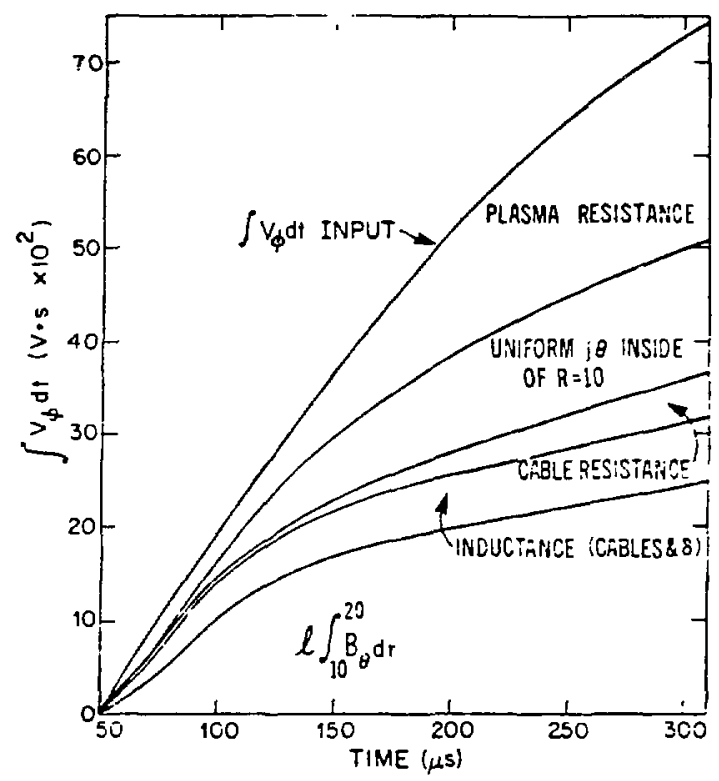

Fig. XV-5.

Plasma resistance as a function of time, deduced from magnetic probe data, circuit resistance component, and volt-second input.

With the assumption that all the components have been calculated correctly, the difference between the sum of the components and input volt-second is due to plasma resistance. At one time the joint resistance ( 68 joints around the primary) was thought to be important, but measurements showed that their effect was small (see below). From the volt-second inventory it is estimated that the plasma resistance was $\sim 3.7$ milliohm. Assuming a $k\left(r_{w} / r_{p}\right) \simeq 2$ and uniform current inside, a plasma resistivity of $1.6 \times 10^{-3} \mathrm{ohm}-\mathrm{cm}$ is calculated and an average $\mathrm{T}_{\mathrm{e}} \simeq 26 \mathrm{eV}$, which is not unreasonable for this mode of operation and relatively low toroldal current.

A second "clean" magnetic field probe has been fabricated of aluminum wire, with the wire surface anodized ( $200 \mathrm{~V}$ insulation) to allow a tight twisting of the leads. The $\mathrm{nA}$ of the coll was $2.5 \mathrm{~cm}^{2}-\mathrm{t}$ and the frequency response was flat, $\leq 2 \%$, to $2 \mathrm{Mhz} / \mathrm{s}$. 
A. Field Perturbations Due to Holes in Primary

This second probe was used first to measure the toroidal $B_{\phi}$ radial distribution along a minor diameter parallel to the major axis of the torus. A hole, $7.3 \mathrm{~cm}$ diameter, in the aluminum primary allowed an extension of the $\mathrm{Al}_{2} \mathrm{O}_{3}$ discharge tube to be connected to the mechanism providing probe motion. The perturbation in the toroidal field of this hole in the primary was measured at a number of radial positions. $B_{\phi}(r)$ is shown in $F i g . X V-6$, normalized to the field on axis. For comparison, the data taken on $2 T-1$ are also shown. It turns out that the ratio of hole diameter to radius of the primary is the same, 0.33 , for the two experiments. The agreement in the two measurements is good, and the results taken with the first aluminum probe, which gave a large field perturbation close to the primary, were in error, as suspected, due to the loose asymmetrical winding of the aluminum leads to the coil.

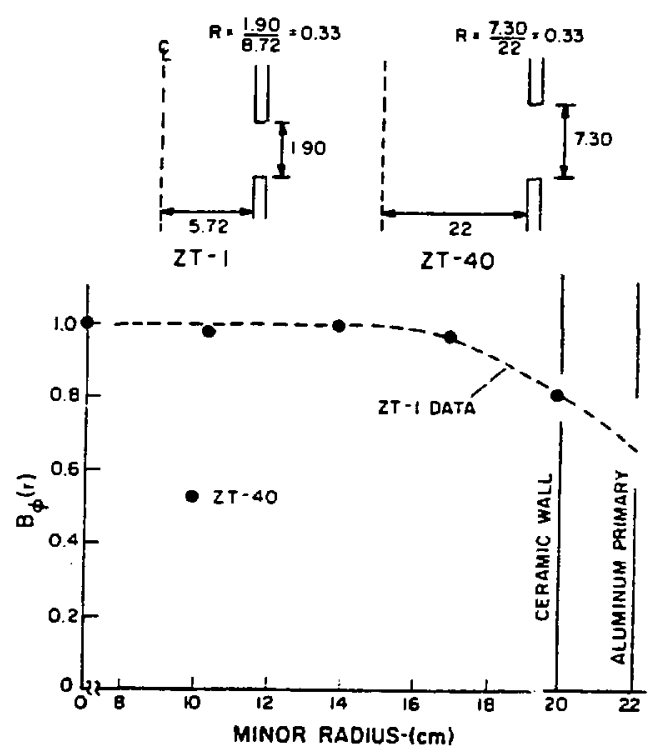

Fig. XV-6.

Toroidal vacuum field as measured by the magnetic field probe on $\mathrm{ZT}-40$ compared with the same data on $\mathrm{ZT}-1$, which has the same geometry for the perturbation in the primary she1l when the probe is inserted. 


\section{B. RFP Magnetic Field Profiles}

To gain some insight in the magnetic field distributions that could exist in $\mathrm{ZT}-40$, these modeis have been examined:

1. a modifled Bessel function (MBF) distribution;

2. $a B_{\phi}(r)$ which falls linearly with radius, ("1inear" mode1);

3. a $B_{\phi}(r)$ with zero slope both at $r=0$ and $r=r(w)$ (wall radius), ("zero slope" model); and

4. a thin-sheath model, $x=r(p)$, with $B_{\phi}$ constant for $r<r(p)$ and equal to $B_{\phi}(W)$ for $r>r(w)$, ("thin sheath" modell).

Plasma pressure has been assumed to be zero in all cases. The poloidal fleld profile $B_{p}(r)$ corresponding to each $B_{\phi}(r)$ model was calculated using this zero-plasma-pressure assumption. To facilitate a comparison between these models and the experimental data, the values of $B_{\phi}(r), B_{\phi}(w), \phi_{\phi}$ (toroidal $\mathrm{flux}$ ), and $\mathrm{B}_{\mathrm{p}}(w)$ are of particular interest.

The MBF model is that proposed by Culham; namely, $\mu$ is constant to a normalized radius of $0.7[r(w)=1]$ and then falls linearly to zero at $r(w)$. An example of this model, $\theta=1.06$, is shown in Fig. XV-7. From a set of these curves (prepared by R. Gribble), the values of interest are determined.

The linear model is simply $B_{\phi}(r)=1-\left[1-B_{\phi}(w)\right] r$, with $B_{\phi}(0)$ normalized to one. $B_{\phi}(r)$ and the calculated $B_{p}(r)$ distributions for four values of $B_{\phi}(w)$ are show in Fig. XV-8.

The zero slope model is defined with $B_{\phi}(r)=\left[1-B_{\phi}(w)\right]\left(1-r^{2}\right)^{2}+B_{\phi}(w)$. Distributions for four values of $B_{\phi}(w)$ are shown in Fig. XV-9. [It turns out that $\phi_{\phi}$ for the two models, linear and zero slope, are identical for the same $\left.\mathrm{B}_{\phi}(\mathrm{w})\right]$.

A comparison of the fleld distritutions for the three models, MBF, linear, and zero slope, for the case $B_{\phi}(w)=0$, is shown in Fig. XV-10.

For the thin sheath model, contrary to the other three, there is not a unique relationship among $B_{\phi}(0), B_{\phi}(w)$, and $\phi_{\phi}$, but a knowledge is required of $B_{p}(w)$ or 0 . In Fig. XV-11, values of $B_{\phi}(0)$ are shown as functions of $B_{\phi}(w)$ for different $\theta^{\prime} s ; \phi_{\phi}$ is held constant.

A normalized set of curves for the first three models, MBF, linear, and zero slope, is shown in $\mathrm{Fig}$. XV-12. In order to compare the experimental data with this figure, the $\phi_{\phi}$ calculated for each model has been modified in the 


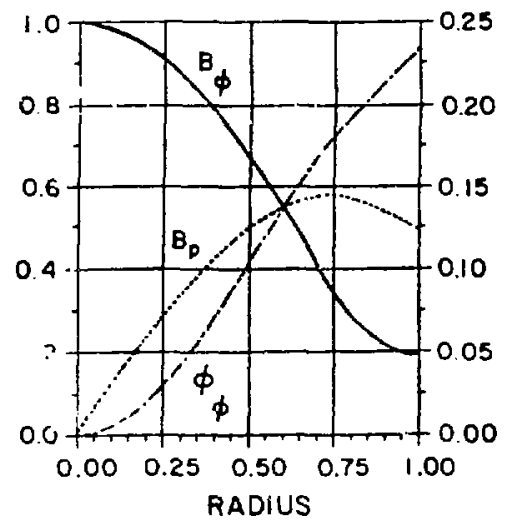

Fig. XV-7.

Magnetic field profiles for the MBF mode1, theta equal to 1.06 .

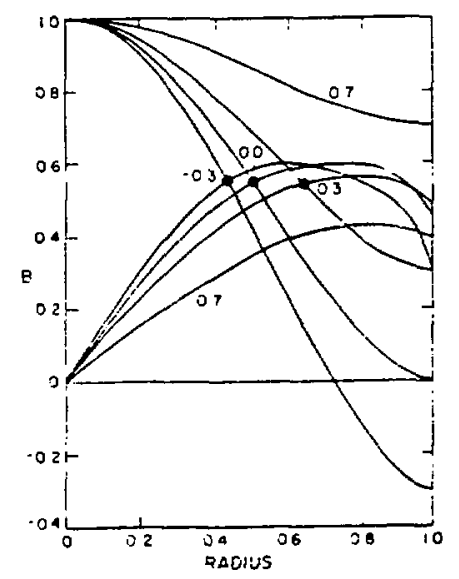

Fig. XV-9.

Field profiles for the zeroslope model.

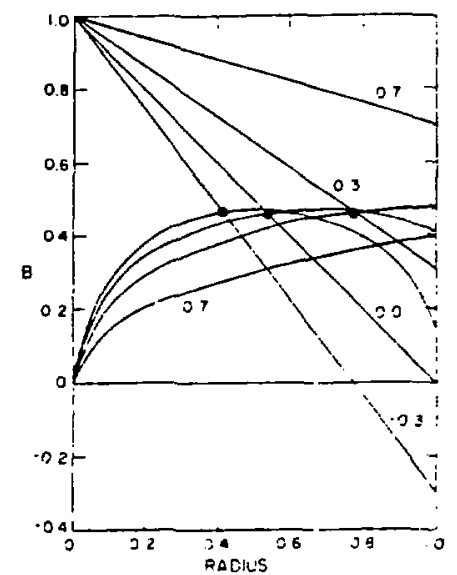

Fig. XV-8.

Magnetic field profiles for the linear model.

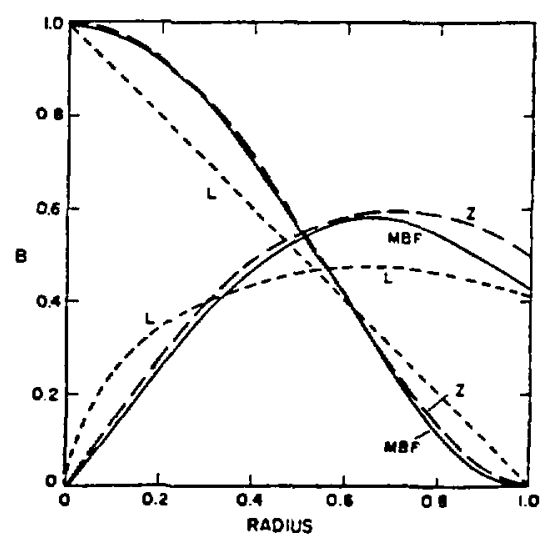

Fig. XV-10.

Magnetic field profiles as a function of radius for the modified Bessel function model, the 1inear model, and the zero-slope model. 


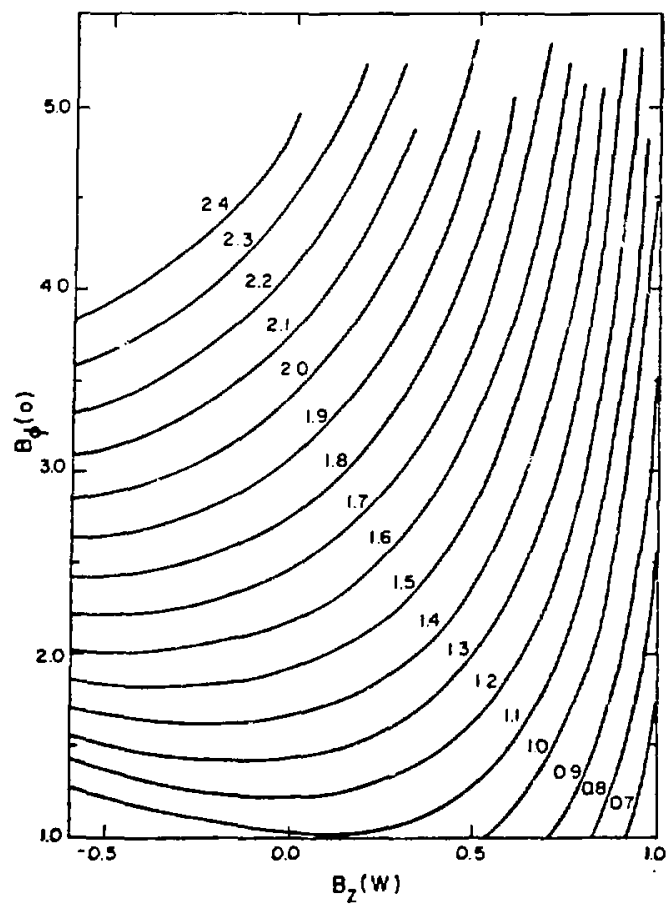

Fig. XV-11.

$B_{\phi}(0)$ as a function of $B_{\phi}(w)$ with $\theta$ a variable parameter. $\phi_{\phi}$ is held constant.

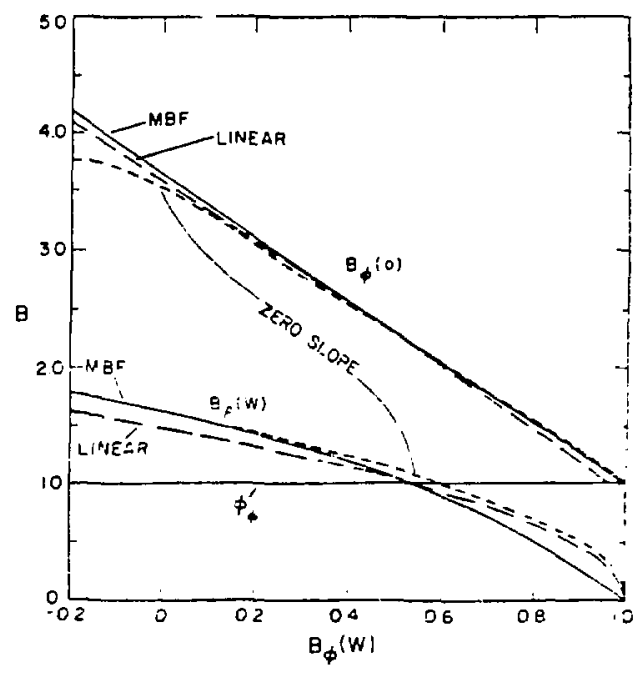

Fig. XV-12. Normalized curves for the three models, MBF, linear, and zero-slope.

figure for the position of the flux loop, $r=21.7 \mathrm{~cm}$, in the experiment. A comparison with the conventional $F$ - $\theta$ diagram can be made in Fig. XV-12, noting that with $\phi_{\phi}=1$, the coordinate $B_{\phi}(w)$ is identically $F$, and $B$ for $B_{p}(w)$ is $\theta$. We see that for $F=0,\left[B_{\phi}(w)=0\right], \theta$ is $\sim 1.53$ for the MBF and zero slope and $\sim 1.48$ for the 1 inear model.

L. Turner ${ }^{2}$ (LT) has calculated field distributions for incompletely relaxed discharges. The equations for the fields are

$$
\begin{aligned}
& B_{\phi}(0)=3.4829-2.4829 B_{\phi}(w) \text { and } \\
& B_{p}(w)=\left(1-B_{\phi}(w)\right)^{1 / 2}\left(2.5384-B_{\phi}(w)\right)^{1 / 2},
\end{aligned}
$$

with $\frac{\phi_{\phi}}{\pi}=1$ 
The variables determined from these distributions are plotted in Fig. XV-13. Comparisons between LT and the other models are not strictly correct since LT has plasma pressure with $\beta \sim 0.20$. The error introduced, however, is considered to be small.

\section{Experimental Data}

A number of discharges were run with the second magnetic probe positioned on the minor axis and rotated to measure $B_{\phi}(0)$. Breakage of the sapphire probe facket prevented a complete radial distribution to be determined.

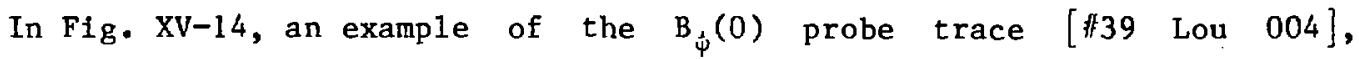
together with those of $\phi_{\phi}[\# 26], I_{\phi}[\# 116]$, and $B_{\phi}(w)[87]$ are shown. The sharp spikes on the magnetic probe signal were identified as electrostatic pickup by opening one of the two probe leads. As seen in Fig. XV-15 (note the different time scales for the two traces), sharp spikes are still present. The origin of these spikes is not understood but may be connected with arcs from the pinch to the steel bellows of the probe system (connected to the primary by $50 \Omega$ ) or by arcs to the pump stands.

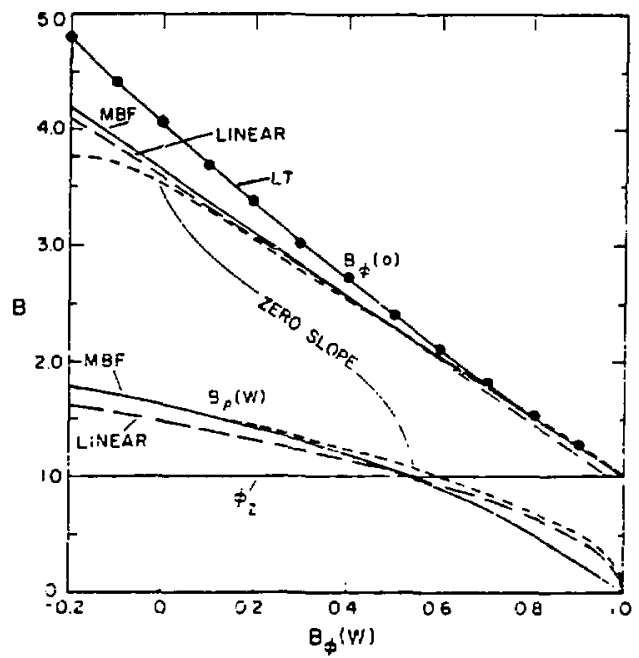

Fig. XV-13.

Turner's result for an incompletely relaxed discharge is compared with the three models, MBF, linear, and zero-slope. 

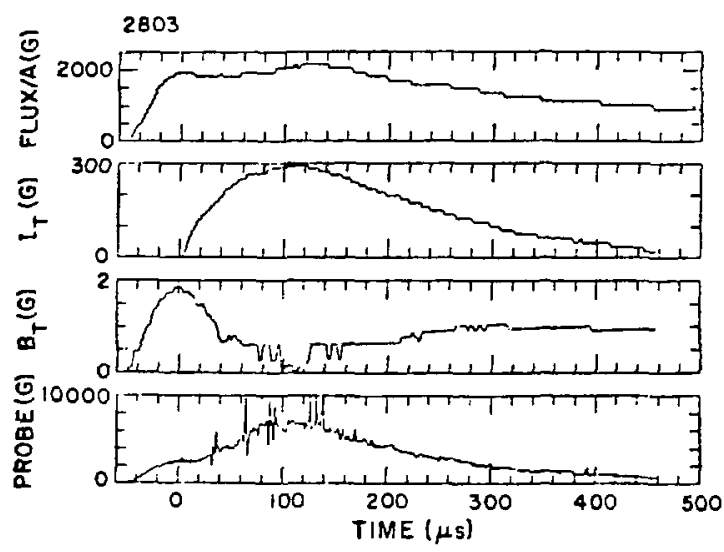

Fig. XV-14.

Comparison of a probe trace with

the toroidal flux, current, and

$B$ at the wall as a function of time.
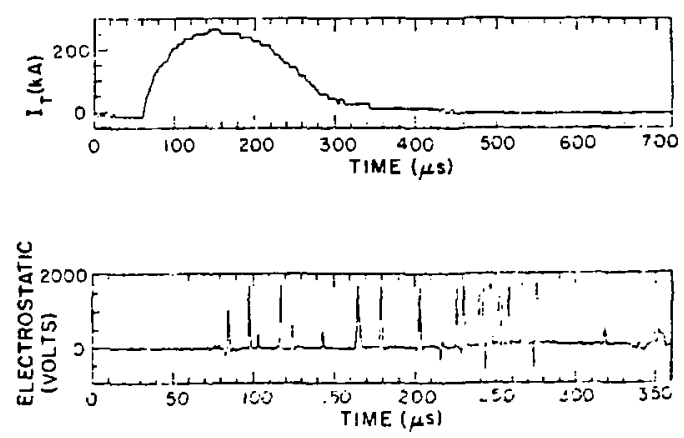

F1g. XV-15.

Electrostatic voltage spikes seen on the magnetic field probe as a function of time compared with the toroidal current. (Note the two time scales.)

\section{Stablized Mode.}

When ZT-40 was run in the stabilized mode, discharges $\# 2802$ and $\# 2803$, the operating parameters were as follows.

$\begin{array}{lcl} & \frac{\# 2802}{1.87 \mathrm{kG}} & \frac{\# 2803}{1.87 \mathrm{kG}} \\ \mathrm{B}_{\phi} \text { initia1 } & 301 \mathrm{kA} & 288 \mathrm{kA} \\ \left.\mathrm{I}_{\phi} \text { maximum (100 } \mathrm{s}\right) & 7 \text { mtorr } \\ \text { Deuterium gas filling pressure } & 10 \text { mtorr } & \end{array}$

The data for $B_{p}(w)$ and $B_{\phi}(\theta)$ versus $B_{\phi}(w)$ [ $\phi_{\phi}$ normalized to one] is shown in Fig. XV-16. The time interval examined was from the initiation of $I_{\phi}$ to $450 \mu \mathrm{s}$ in the discharge cycle.

During the rise, and fall of the toroldal current, both $B_{p}(w)$ and $B_{\phi}(0)$ appear to follow in general the same curve; $1 . e$. , there is no clear difference in the behavior of these variables during the falling currents from those on the current rise. (Conventionally, on a $F-\theta$ diagram the experimental points cluster, in general, about a single curve.)

$B_{p}(w)$ is in fair agreement with the predictions of the models for $B_{\phi}(w)$ $\leq 0.5 \mathrm{~cm}$ and are $h t g h e r$ for $B_{\phi}(w) \geq 0.5 \mathrm{~cm}$. This may be understood by noting that the $B_{p}$ field diffuses to the axis at early times $\left(B_{\phi}(w) \geq 0.5 \mathrm{~cm}\right)$ and there will be significant differences with the models. 


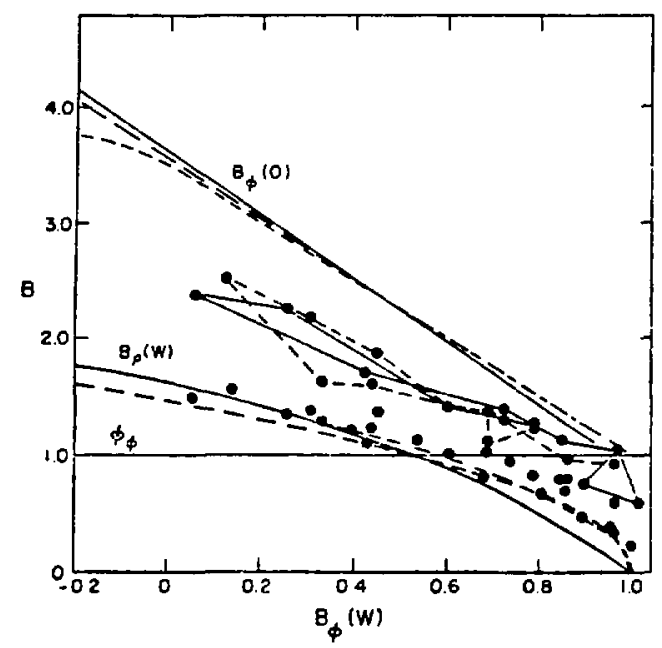

F1g. XV-16.

$B_{p}(w)$ and $B_{\phi}(0)$ plotted against $B_{\phi}(w)$ for two discharges of ZT-40 when run in the stabilized mode. The smooth curves are the distributions predicted by the theoretical models as shown in Fig. 13. $B_{p}(w)$ follows closely the theoretical curves but the $\mathrm{B}_{\phi}(0)$ is considerably lower.

Values of $B_{\phi}(0)$ (indicated by straight lines connecting the experimental points) are significantly smaller, $\sim 40 \%$, than predicted. A number of suggestions have been made to explain the differences.

1. The experimental $B_{\phi}(r)$ distributions are considerably flatter than the models considered.

2. The sapphire jacket under the intense plasma bombardment of long time duration is surrounded by a conducting layer shielding out rapid variations in the field. (This is our first experience with probes in discharges lasting hundreds of microseconds.)

3. The equilibrium position of the pinch is displaced radially outward from the major axis, as predicted by theory and seen in other toroldal experiments, and the magnetic field probe on axis does not measure the peak $B_{\phi} \cdot$ An asymmetric distribution would, however, not merely displace the peak $B_{\phi}$, but would modify the relationship between the parameters $\phi_{\phi}$, $B_{p}(w)$, and $B_{\phi}(w)$. 
2. Alded Mode.

When $\mathrm{ZT}-40$ is run in the aided mode, $\mathrm{B}_{\phi}(\mathrm{w})$ passes through zero. The operating parameters were as follows.

$$
\begin{aligned}
& \mathrm{B}_{\phi} \text { peak }-2.15 \mathrm{kG} \\
& \mathrm{I}_{\phi} \text { maximum }(100 \mu \mathrm{s})-274 \mathrm{kA} \\
& \text { Deuterium gas filling }-7 \text { mtorr }
\end{aligned}
$$

The data for two discharges, \#2805 and \#2806, are shown in Fíg. XV-17. $B_{p}(w)$ follows the same behavior as in the stabilized mode. $B_{\phi}(0)$ shows, however, a significant difference from the stabilized mode above. After $B_{\phi}(w)$ passes through zero and becomes negative, $B_{\phi}(0)$ markedly increases and comes close to (and sometimes above) the predicted values. From the limited data it is impossible to say if there has been a shift in the equilibrium position when $B_{\phi}(w)$ is small or negative (moving from a position outside the magnetic axis towards the magnetic axis); no correlation, however, with other diagnostics indicating radial motion has been found. The data do seem to indicate that the probe may not be seriously affected by the discharge. These preliminary results do indicate that the position of the discharge magnetic axis is important.

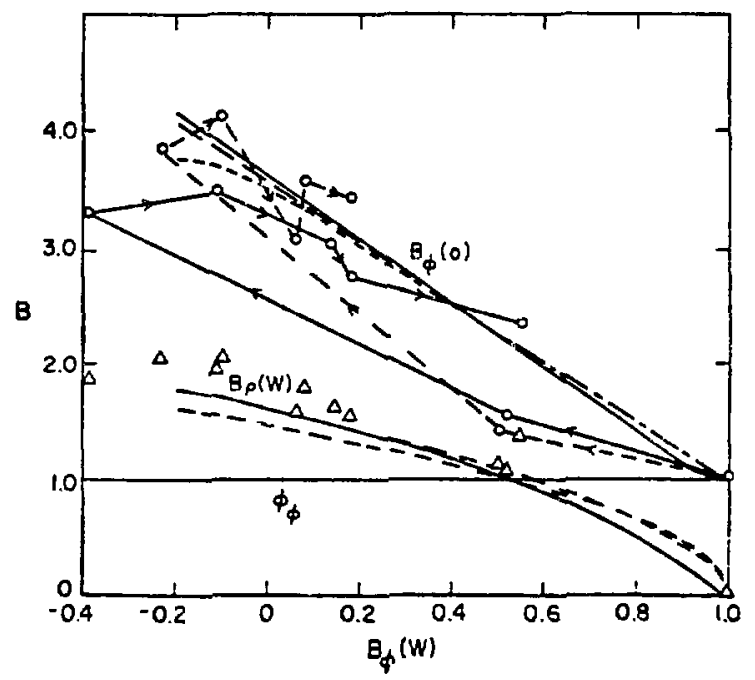

Fig. XV-17.

Aided mode. Note that when run in this mode, the $B_{\phi}(0)$ is below the theorettcal predictions until after $B_{\phi}(w)$ passes zero. 
IV. CONCLUSION

Comparisons of the preliminary experimental data with the models show that we cannot exclude any of the models at this time.

\section{REFERENCES}

1. S. Glasstone and R. Lovberg, Controlled Thermonuclear Reactions (D. Van Nostranc and Co., Inc., New York, 1960), p. 237.

2. L. Turner and J. P. Christiansen, "Incomplete Relaxation of Pinch Discharges," Phys. Fluids 24, No. 5 (May 1981), pp. 893-898. 
CHAPTER XVI

HELICAL COII MEASUREMENTS

by

G. Mi1ler

These measurements used the hellcal pickup coll shown in Fig. XVI-1. The coil was divided into four segments because of anticipated problems with high voltage, although this was not actually necessary. A detected signal (the sum

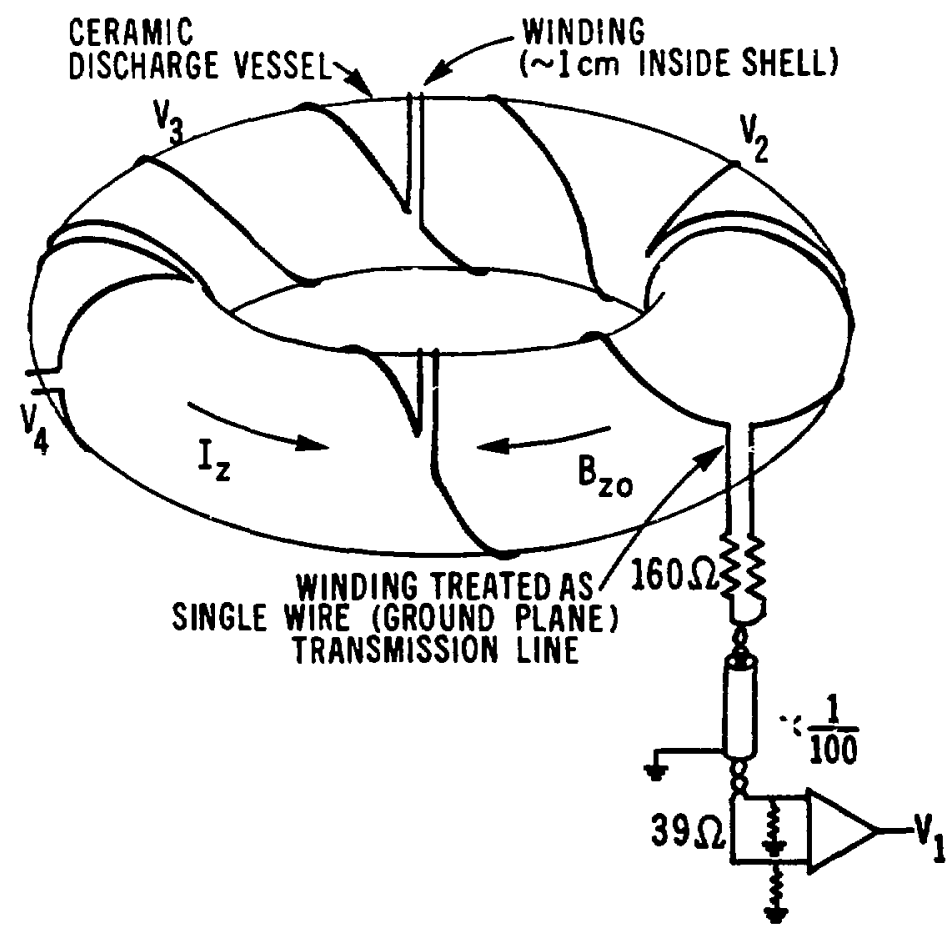

F1g. XVI-1.

Helical pickup col1. 
of the signals from the four segments) Implies the presence of magnetic field fluctuations having $B_{r} \sim \sin [m(\theta-4 z / R)]$, where $R$ is the major radius of the torus and $\mathrm{m}=1,3,5 \ldots$ To simplify we assume $\mathrm{m}=1$ dominates, as would be expected from stability theory. In this case the magnitude of the fluctuating fleld at the conducting wall is given by

$$
|\delta B|=\frac{1}{4 \pi \Delta R\left(m^{2}+n^{2} a^{2} / R^{2}\right)^{1 / 2}} \int V d t_{t},
$$

where $\Delta$ is the distance from the discharge tube to the conducting wall, $m=1$, $\mathrm{n}=4$, a is the minor radius of the torus, and $\mathrm{V}$ is the induced voltage. The direction of the detected fluctuating field is parallel to the conducting wall and perpendicular to wires of the coll. A set of colls of this type could conveniently resolve the field fluctuations into $m$ and $n$ components and the single coll was meant as a first step toward a more complete measurement.

It is natural to question the frequency response of such a coil because of its large size. To improve the frequency response, each wire is operated as a transmission line, with the conducting wall providing a ground plane. The characteristic impedence was determined empirically to be around 200 ohms. A frequency response check was dorie by threading the pickup loop through a ferrite core having several turns driven by a pulse generator. A square pulse was distorted only in the leading and trailing $0.2 \mu \mathrm{s}$ of the pulse. This implies a bandwidth of at least $1 \mathrm{MHz}$.

Roughly speaking, the measurements showed the following: the voltage began erratic fluctuations with an amplitude on the order of $\pm 1 \mathrm{kV}$ a short time after the start of the current; the amplitude was variable but quiet periods have not been observed, even with a reversed field; the fluctuations were slower at higher filling pressures, and the converse.

During two dedicated runs the sign of $B_{z O}$ (see Fig. XVI-1) was reversed to provide the equivalent of $n=-4$ as well as $n=+4$ data. For the first of these runs the machine was operated in the Tokamak regime to Investigate the delay in the onset of turbulence. Figure XVI-2 shows the rotational transform, or $1 / q=R B_{\theta} /\left(a B_{z}\right)$, as a function of time for a series of shots. These curves may also be thought of as giving the time dependence of the 


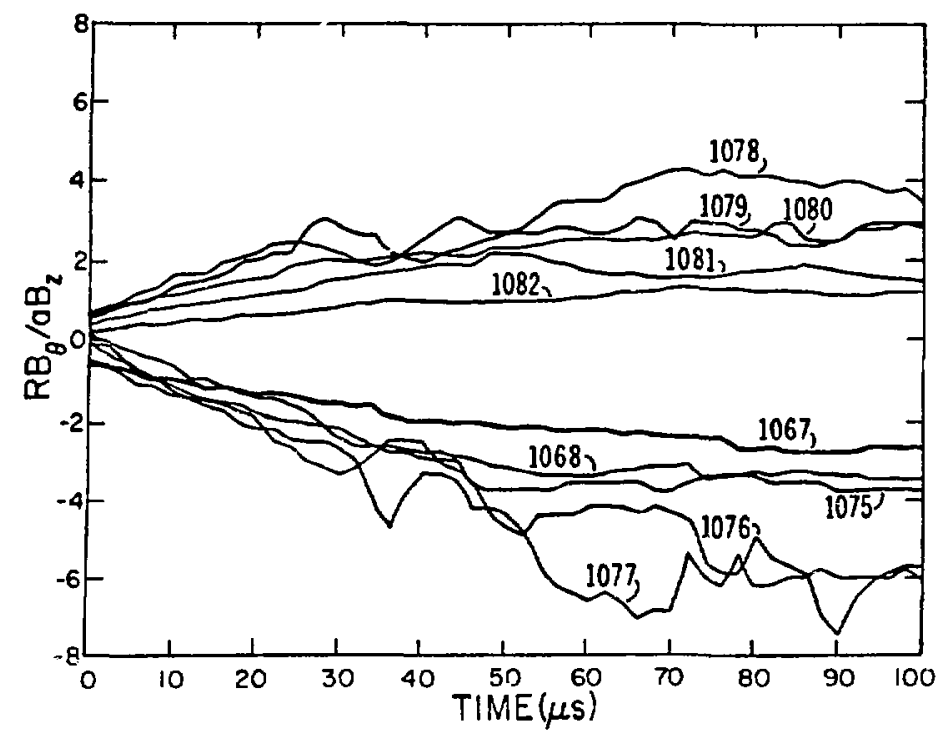

Fig. XVI-2.

Rotational transform for a series of low-current shots.

current, since $B_{z}$ is essentially constant. Some error is present because the magnetic probes used to determine $I_{z}$ had some $B_{z}$ pickup.

The results are given in Fig. XVI-3, which shows the voltage and $\delta B / B$ [obtained from fVat using Eq. (1)] for the shois shown in Fig. XVI-2. For $\mathrm{n}=-4$, which by convention means that the fleld lines at the wall rotate in the same sense as the pickup coll windings (shots 1067-1077), there is a fairly well defined threshold for $1 / q=3 \pm 1$. The flactuation level is large, up to \pm 0.4 (using the nominal value of $1 \mathrm{~cm}$ for $\Delta$ ). For $\mathrm{n}=+4$ the turbulence level is lower and the onset rotational transform smaller. From the interferometer, the onset of erratic density flictuations seems to occur at rotational transform values $\lesssim 1$.

The second $n= \pm 4$ run investigated the reversed-field configuration. (This data was actually taken during one feedpoint operation of $\mathrm{ZT}-40$, but this seems irrelevant.) Figure XVI-4 shows the $I_{z}$ and $B_{z}$ histories for two shots where 1 t was attempted to have everything the same except the sign of $\mathrm{B}_{z} / \mathrm{I}_{\mathrm{z}}$. The helical coil signals during the period of field reversal are shown in Fig. XVI-5. The $n=-4$ signals are perhaps larger, but the basic observation is that $n= \pm 4$ are approximately the same, in contrast to the Tokamak case. 
Fig. XVI-3.

(a) Measured helical coil voltage.

(b) Fractional magnetic field fluctuation level for shots in the low-current serles.
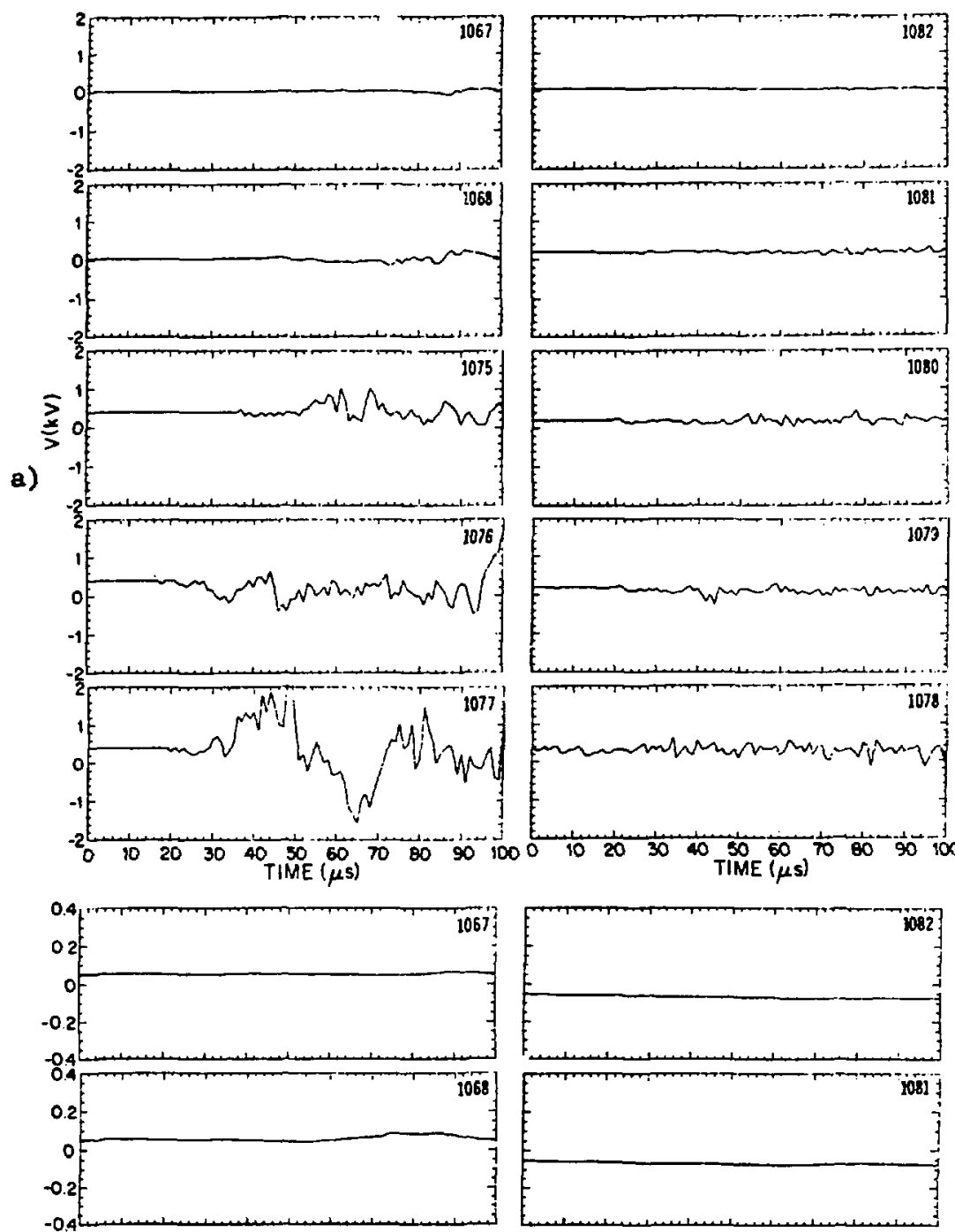

b)
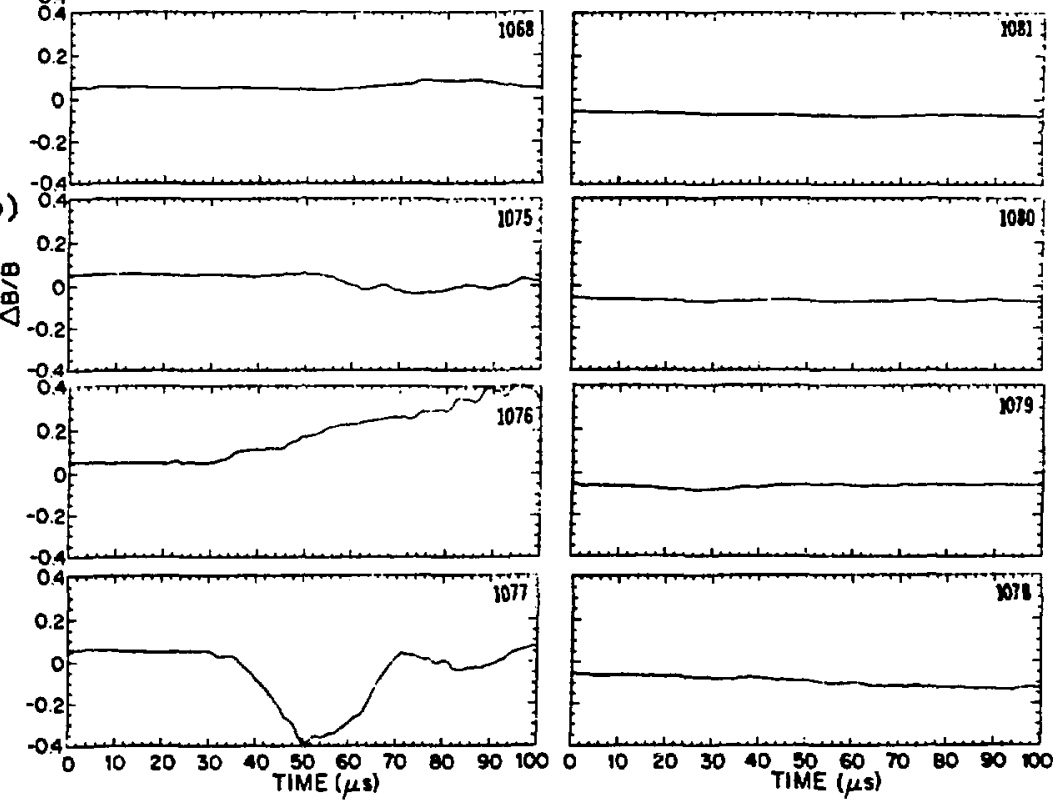

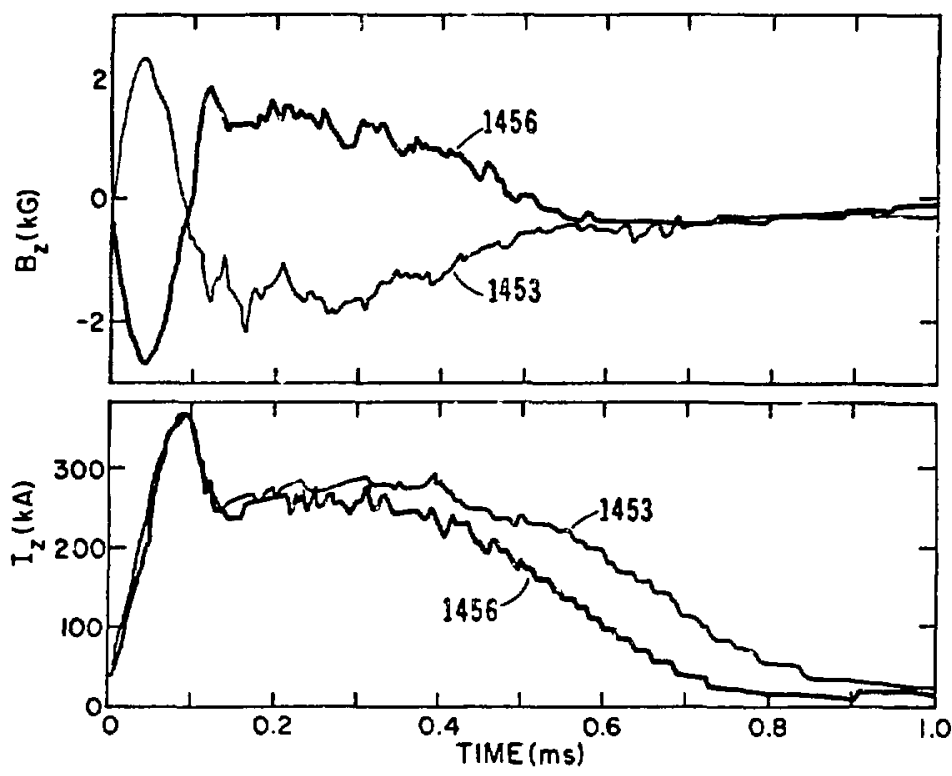

Fig. XVI-4.

$B$ and $I_{z}$ histories for the $n= \pm 4$ shots.

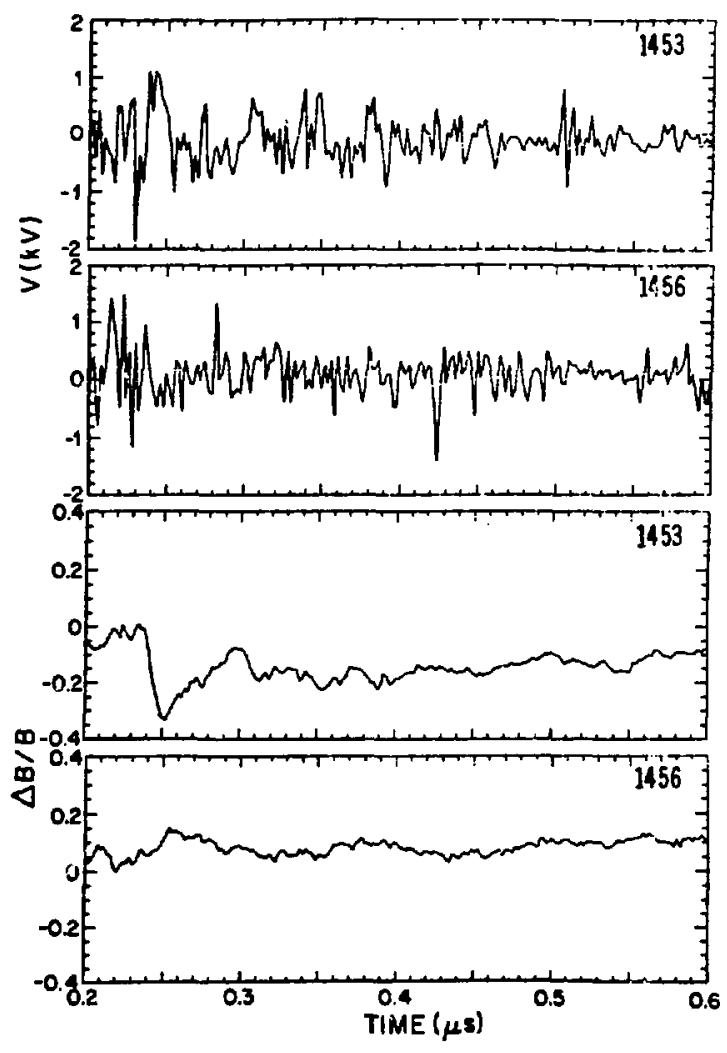

Fig. XVI-5.

Voltage and fleld fluctuation level for $n=-4$ (top)

and $n=+4$ (bottom). 
Studies were also made of the separate signals from the four segments of the complete coil. These signals showed very little correlation. The only indication of the proper phasing was for the Tokamak runs with the equilibrium field approximately aligned with the coil windings. In this case the sum signal was slightly larger for 1,1,1,1 phasing. Generally, however, the sum signal was largest for $1,-1,1,-1$ phasing. This 1 s probably due to the presence of $m=0$ modes which would be picked up by the semicircular parts of the coil segments. This pickup cancels for $1,1,1,1$ phasing. It is not surprising to have $m=0$ present since it, as well as $m=1$, is 11 kely to be a dominant unstable mode. 
CHAPTER XVII

UV SPECTROSCOPIC OBSERVATIONS

\author{
by \\ ‥ E. Howe11
}

\title{
I. INTRODUCTION
}

Spectroscopy is being used on $\mathrm{ZT}-40$ to provide information on the electron temperature, the impurity type and concentration, and the dependence on discharge conditions. The electron temperature and lensity in the plasma will determine the time evolution of impurity ionization states. An atomic physics code written by ortolani and Jahoda solves the rate equations for the Ionization states. One version, $0 . \mathrm{J} .2,1$ computes the electron temperature with the ohmic heating and losses competing, coupled with the circuit for the toroidal current. The dominant radiation loss comes from line radiation and, in particular, the resonance 1ines. These lines are found in the vacuum ultraviolet (VUV) part of the spectrum (below $2000 \AA$ ). It is well.known ${ }^{2}$ that for even small impurity concentrations, the radiation barrier can severely limit the maximum temperature achieved. There is a barrier around $30 \mathrm{eV}$ due to low $Z$ impurities, 1ike oxygen. For high $Z$ impurities, 11 ke 1 ron and aluminum, there is a barrier above $100 \mathrm{eV}$.

The most important short-term use of the spectroscopic data is to help establish the $\mathrm{ZM}-40$ conditions for the highest temperature and the longest duration of confined plasma. The longer term goal is to better understand the plasma processes.

An important part of this is to obtain emission data as functions of parameters 11ke maximum $\theta=B_{\theta}$ (wall)/ $\left\langle B_{\phi}\right\rangle$, filling pressure, and different toroldal currents. Fmission data have problems of interpretation such as lucation of emitting region, changing impurity concentration, and radial variations. 
There are two spectrometers used for these emission measurements on ZT-40. The VUV instrument is a $1-\mathrm{m}$ normal incident spectrometermonochromator, with a $600 \mathrm{l} / \mathrm{mm}$ platinum-coated grating. It has a dispersion of $16.6 \AA / \mathrm{mm}$ and entrance slits of $10,20,50$, and $100 \mathrm{\mu m}$. The usefu1 lower wavelength $11 \mathrm{mit}$ is approximately $300 \mathrm{~A}$. The second instrument is a $1-\mathrm{m}$ Czerny Turner spectrometer-polychromator, which has a lower wavelength 11mit of $2000 \AA$.

\section{LINE EMISSION AS A TEMPERATURE DIAGNOSTIC}

The time development of the lonization states can provide a tool for determining the electron temperature. The time evolution has been calculated by simply solving the rate equations for a given temperature and density. This was done with another version of the atomic physics code developed by Jahoda, Ortolani, and Mann (0.J.1). 3 The $0 . J .1$ code was modifled to include a changing electron temperature vs time along with a packaged plotting routine. 4 An example of the calculated $O$ VI time development is shown in Fig. XVII-1b for three cases of a changing electron temperature shown in Fig. XVII-1a. The time behavior has been quantified by measuring the full

(a)

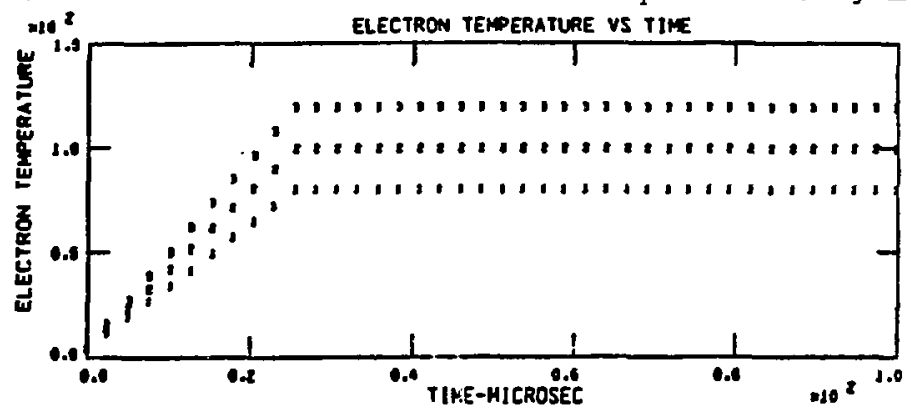

(b)

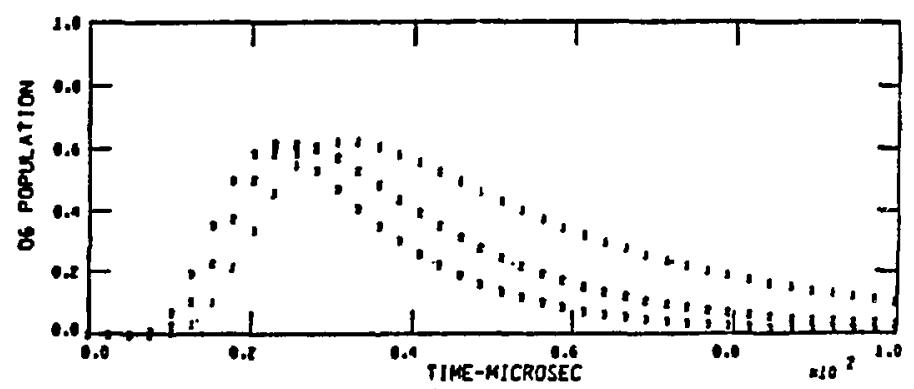

Fig. XVII-1.

(a) Three cases of electron temperature as a function of time. The maximum temperature, $T_{M}$, reached in each case is 80,100 , and $120 \mathrm{eV}$, respectively. (b) The time development of the 0 VI lonization state for the three cases in $1(a)$. 
time width $t_{w}$ at the e-folding time for the o vi ionization state. The maximum temperature $T_{M}$, with ramp times of $25 \mu \mathrm{s}$ and $50 \mu \mathrm{s}$, is plotted vs $t_{\mathrm{w}}$ in Fig. XVII-2 for a density of $4 \times 10^{14} \mathrm{~cm}^{-3}$. The ramp time is the time for the temperature to rise linearly from $5 \mathrm{eV}$ to the maximum $T_{M}$. These rise times for the temperature are postulated to represent the temperature change .. in $2 \mathrm{~T}-40$.

The density dependence of $t_{w}$ is shown in Fig. XVII-3. The band for each temperature represents the spread in $t_{w}$ calculated by using the two ramp times of 25 and $50 \mu \mathrm{s}$. The time development of the 0 VI Ionization state shown in Fig. XVII-1b is characterized as a burnthrough.

The use of $t_{w}$ vs $n_{e}$ to determine electron temperature has these requirements :

1. The atomic physics coefficlents used in the Ortolani-Jahodal code are sufficiently accurate.

2. The electron temperature has a time dependence as postulated above.

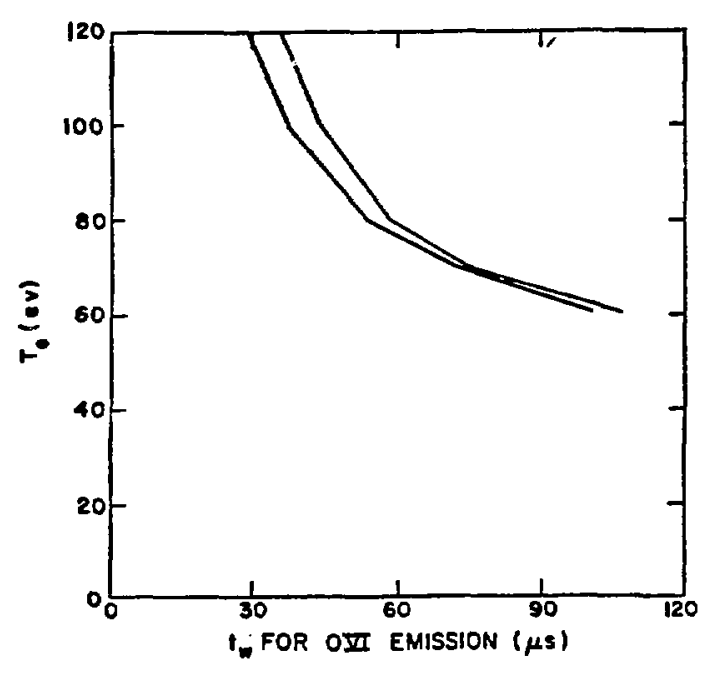

Fig. XVII-2.

The maximum temperature $\mathrm{T}_{\mathrm{M}}$ used to obtain the time $t$ for 0 VI at $n_{e}=4 \times 10^{14} \mathrm{~cm}^{-3}$

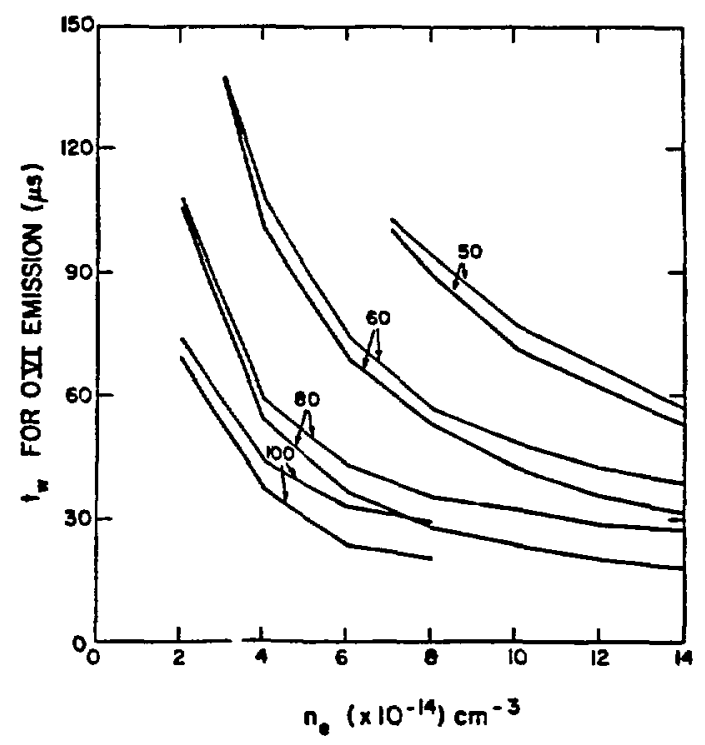

F1g. XVII-3.

Loc1 of constant $t_{w}$ for ramp times of 25 and $50 \mu s$. The numbers assoclated with each pair of lines represents the maximum temperature $T_{M}$. 
3. Certain experimentally obtained information is necessary to determine what constitutes a burnthrough as opposed to simply a decrease in cbserved intensity.

The following conditions are necessary for burnthrough to be determined from observed line emission of an ionization state $N$.

1. The resonance Iine must be observed.

2. The region containing the ith state must not be moving into and out of the fleld of view.

3. The spatial density dependence must be known for the electrons and the ions in the Nth state.

4. No influx into or out of the region of emission can occur for states other than $\mathrm{N}$.

5. The electron temperature must not decrease below the excitation level of the upper state of the resonance line.

Condition 1 should always be fulfilled. Conditions 2 and 3 can only be fulfilled by observing several chords of the plasma simultaneously at the wavelength of the Nth state. This has not been done in the present measurements. Condition 4 would be very hard to fulfill under any circumstances. Condition 5 should be satisfied in most cases for 0 VI because the excitation energy for the upper state of the resonance line is only $12 \mathrm{eV}$.

With these severe conditions on the determination of burnthrough, there is very little hope of making that unequivocal determinaioion. However, it should be possible to use the raw emission as a guide to the temperature in the plasma.

\section{SETUP OF PLASMA}

For the spectroscopic measurements presented here, the methods of programming were the self-reversal and aided-reversal modes. The plasma conditions used for results given here are

$$
\begin{aligned}
& I_{\phi}(\text { peak }) \simeq 500 \mathrm{kA} \text { and } \\
& \theta_{\mathrm{m}}=1.4 \text { to } 2.5,
\end{aligned}
$$


where $\theta_{\mathrm{m}}$ is the maximum $\theta$ in the discharge and is usually found at the crowbar time of $\mathrm{B}_{\phi}$ in the mode of urrent programming used here. A typical set of current and voltage waveforms is seen in Fig. XVII-4, for the alded-reversal mode.

IV. IMPURITIES PRESENT

The impurity spectrum in $2 \mathrm{~T}-40$, in the range 500 to $1100 \mathrm{~A}$, is shown in Fig. XVII-5. This spectrum was taken with a filling pressure of 5 mtorr. The main impurities appear to be oxygen, carbon, and nitrogen, with traces of aluminum appearing in certain conditions. In the UV region the most prominent lines are $O \mathrm{VI}$ and $O \mathrm{~V}$.

The level of oxygen impurity was measured for the 5-mtorr condition. The data for this are showr in Fig. XVII-6. This measurement obtained $\sim 1.25 \%$ oxygen in the discharge.

Attempts were made to measure the amount of nitrogen impurity. The nitrogen level measured for the 7-mtorr condition was $\leq 1 \%$ up to 40 us into the discharge. After this the nitrogen level tended to fluctuate too much for an impurity determination. However, from the $N$ IV (1238 \&) intensity measurements and the CV $(2271 \AA)$ intensity measurements, it takes approximately 5 or $6 \%$ nitrogen adied to the plasma to cause losses equivalent to the energy losses already present.

V. LINE EMISSION DATA

The data presented here use the programmed $B_{\phi}$ reversal mode. Examples of 0 VI (1032 \&) intensity from the VUV monochromator are shown in Figs. XVII-7a and 7b. The signal in Fig. XVII-7a does not appear to represent "burnthrough" even if the plasma is assumed to be homogeneous and uniform (satisfying conditions 3 and 4 and also conditions 2 and 5 ). The data are quantified as mentioned above, by measuring the fill time width of the e-folding time for the detected $0 \mathrm{VI}$ intensity. In Fig. XVII-7b, the e-folding time is 47 us for the first peak, which appears to represent burnthrough. The second peak elther represents the cooling off of the plasma or a wall hit with preionization of new $0 \mathrm{VI}$ taking place. The quadrature interferometer (Q. I.) signals 5 in units of wavelength phase shifts are given In Figs. XVII-8a and 8b, corresponding respectively to Figs. XVII-7a and $7 \mathrm{~b}$. 


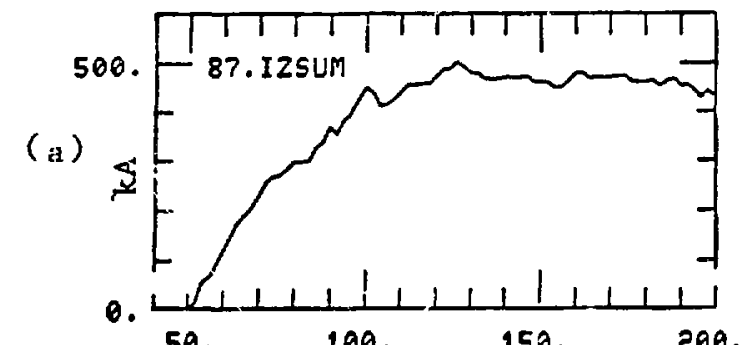

TIME INTEGRATED

(b)
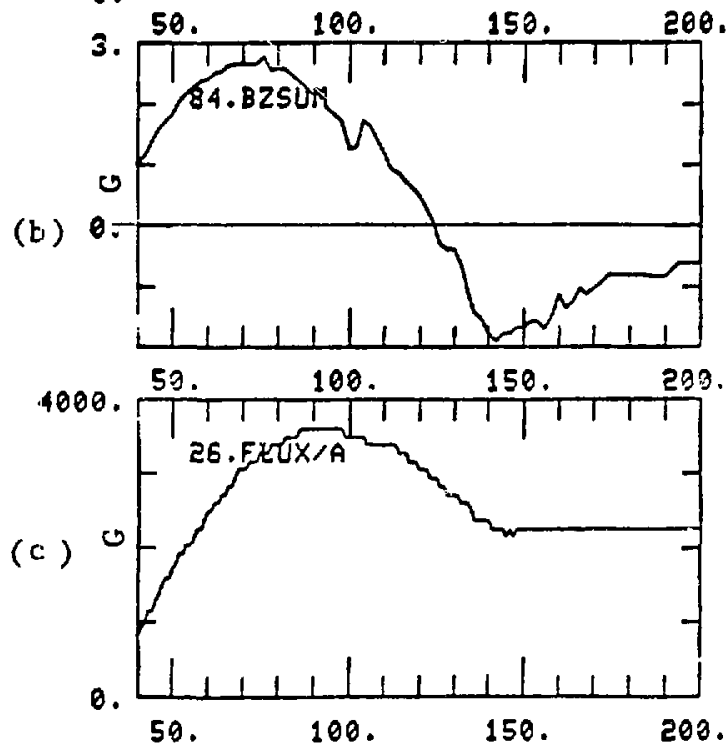

290.

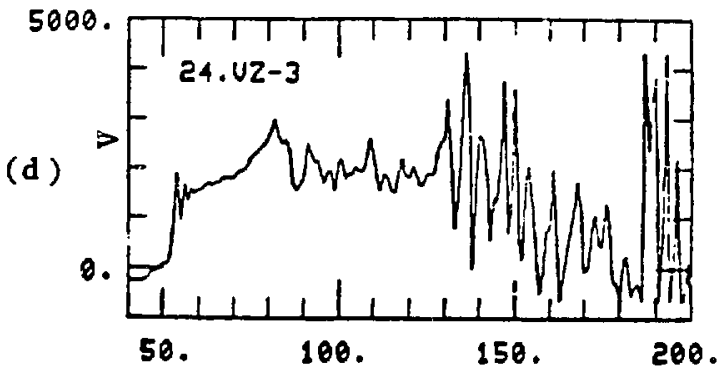

FIg. XVII-4.

Here are shown, respectively, toroldal current $I_{\phi}$, toroldal magnetic field $B_{\theta}$ at wall, average $B_{\phi}$, voltage on ${ }^{m}$ feedplates.

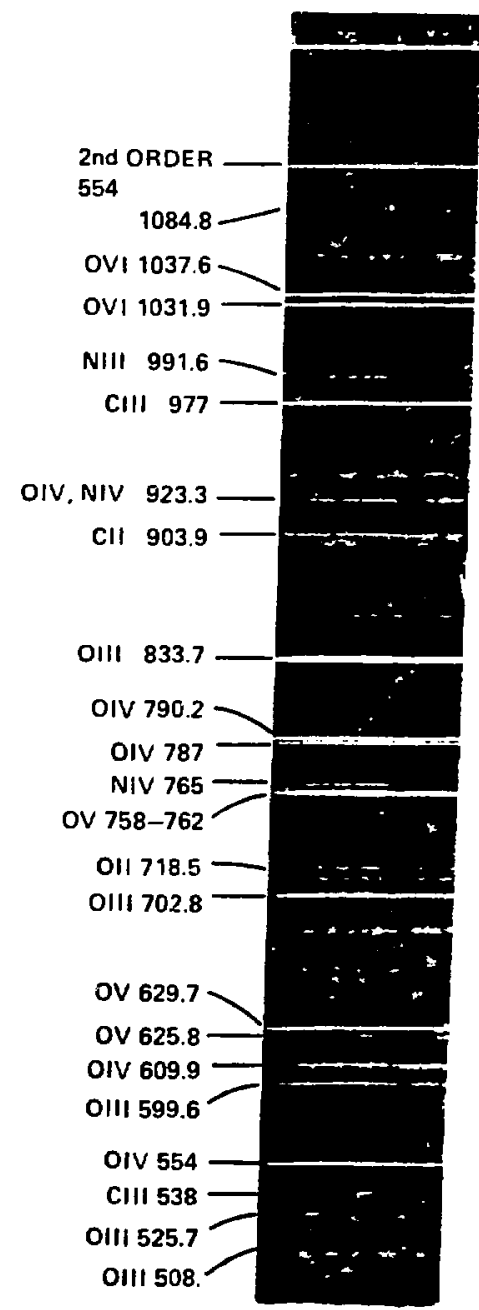

Fig. XVII-5.

Impurity spectrum between $500 \AA$ to $1100 \AA$.

In this picture, there are quickly changing amplitudes ( $\sim \mathrm{MHz}$ ) and slowly changing amplitudes $(\sim 10-50 \mathrm{kHz})$. The slower interferometry signal behavior is on the same time scale as the change of the $0 \mathrm{VI}$ emission. In Fig. XVII-8b, the signal, defined by a fast fluctuating envelope, remains 


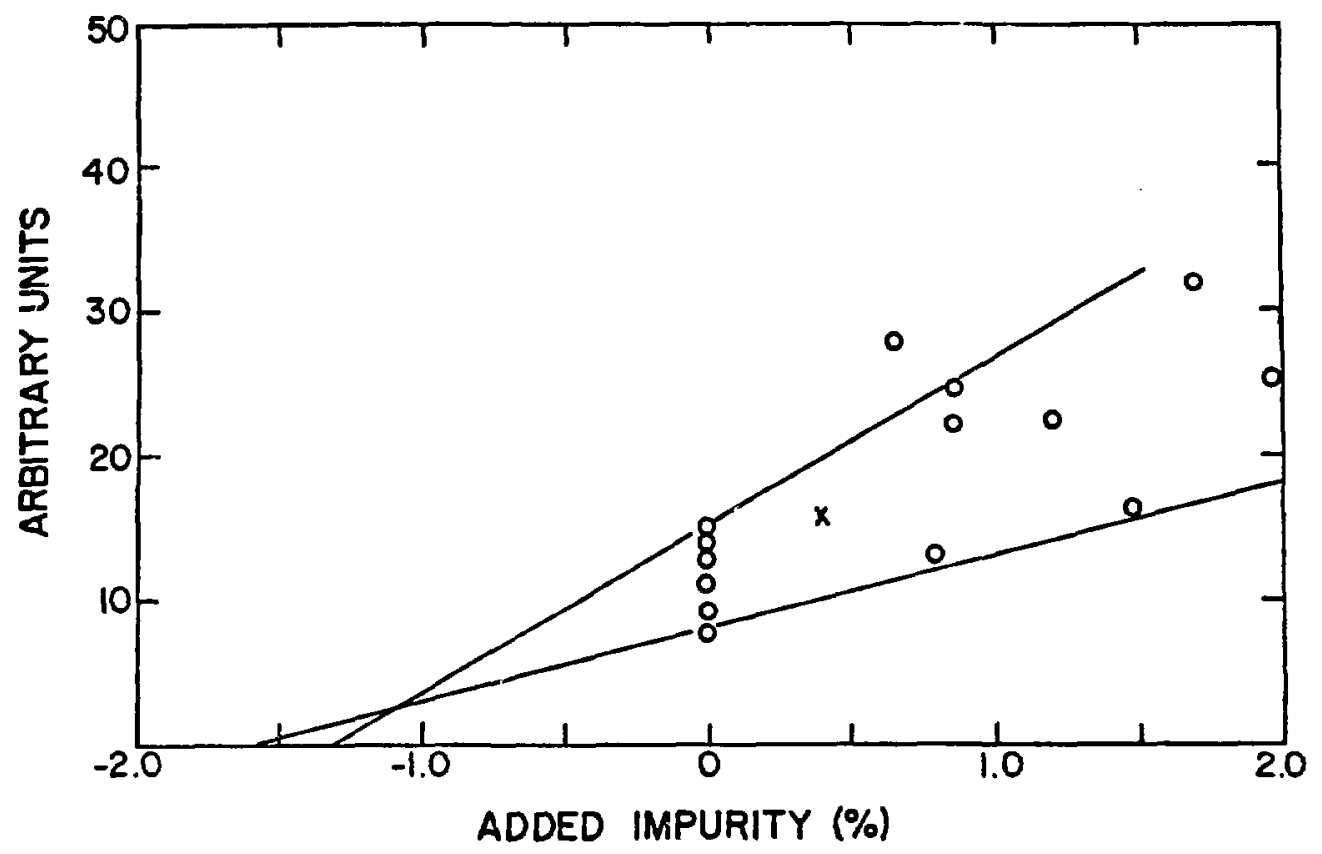

Fig. XVII-6.

O III line intensity integrated over discharge time for different percentages of added oxygen tmpurity.

(a)

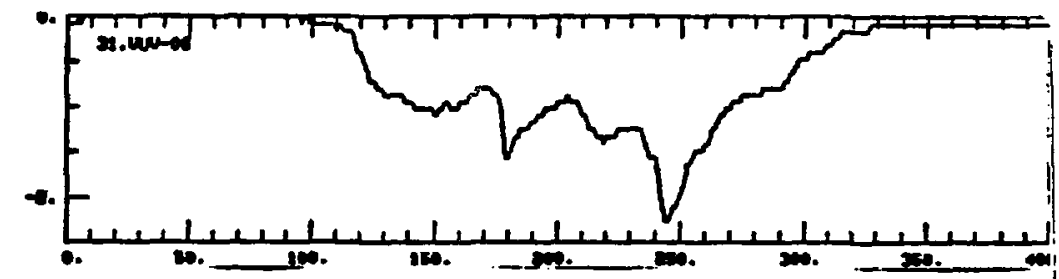

(b)

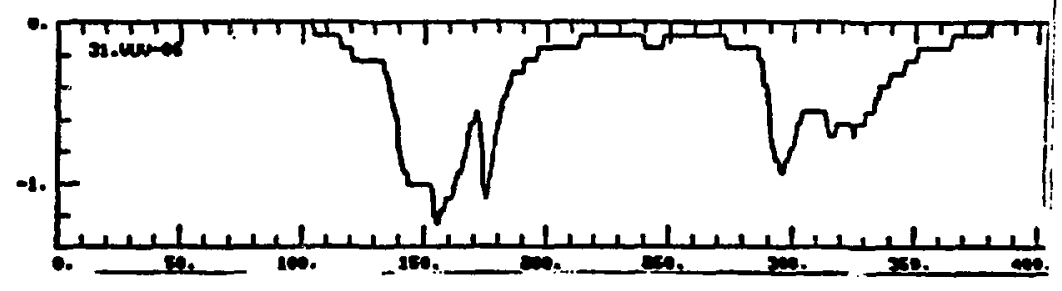

Fig. XVII-7.

0 VI (1032 A) Iine intensity for two plasma discharges. 


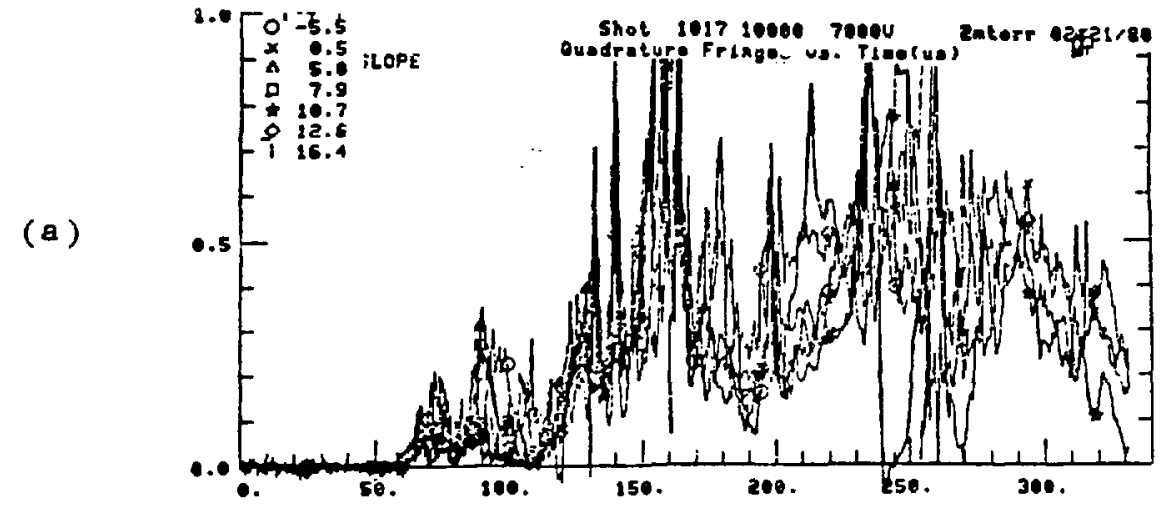

(b)

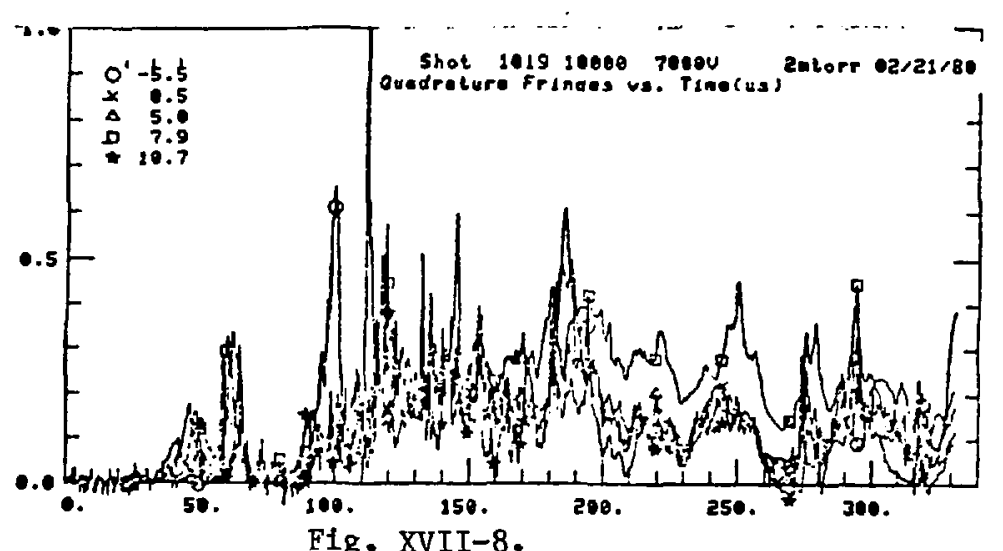

Quadrature interferometer signals for case $7(a)$ and $7(b)$, respectively.

essentially constant from $150 \mu \mathrm{s}$ to $170 \mu \mathrm{s}$, dips down at $175 \mu \mathrm{s}$ and then increases to about twice its original value. Thus, the decreased intensity of 0 VI In Fig. XVII-7b should not be due to a decrease in the electron or ion density. This assumes the density at the location of the interferometer is the same at the location of the VUV spectrometer.

A further example of $0 \mathrm{VI}$ IIne emission along with an $0 \mathrm{~V}$ line (not the resonance 1ine) is shown in Figs. XVII-9b and 9c. The current pulse for this discharge is shown in Fig. XVII-9a. 
(a)

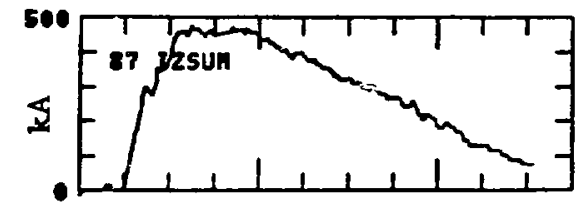

(b)

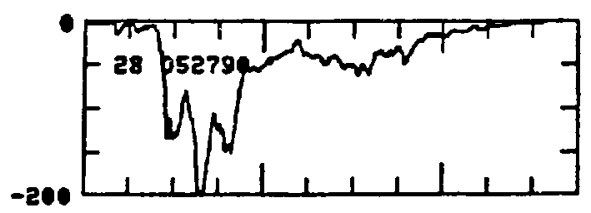

(c)

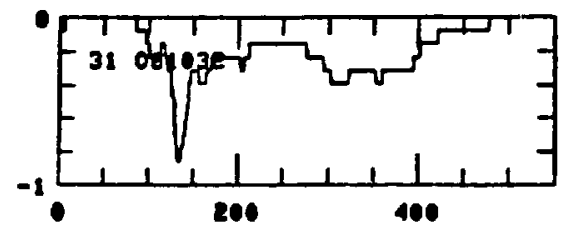

Fig. XVII-9.

(a) Toroldal current waveform. (b) $0 \mathrm{~V}$ line intensity (2790 A).

(c) O VI line Intensity (1032 \&).

VI. PARAMETER SCALING OF $t_{w}$

A. $t_{w}$ vs $\theta_{\mathrm{rn}}$

The full width time $t_{w}$ for $0 \mathrm{~V}$ was measured for different values of $\theta_{\mathrm{m}}$. This full width time is shown in Fig. XVII-10 for a filling pressure of 20 mtorr. Measurements were thrown out when the density decreased rapidiy near the time of the falling intensity of $0 \mathrm{~V}$. These were discarded because In those cases the decrease of the $0 \mathrm{~V}$ signal may be due to decreasing electron density. The data in Fig. XVII-10 can be used to estimate temperature based on the time evolution as computed with the code mentioned above. Frrin the numerical calculations, the data in Fig. XVII-10 indicate temperature less than $40 \mathrm{ev}$. There also appears to be a tendency for the temperature to increase as $\theta_{\mathrm{m}}$ increases.

The full width $t_{w}$ of $O$ VI is shown in Fig. XVII-11. For the 20 mtorr condition there is a tendency for $t_{w}$ to decrease as $\theta_{\mathrm{m}}$ increases. Since the electron density exhibits no increase with increasing $\theta_{m}$, there is an indication of higher temperatures as $\theta_{\mathrm{m}}$ increases. If these signals represent partial or complete burnthrough for the najority of the plasma in the fleld of view of the spectrometer, the ratio of the maximum signal to the minimum 


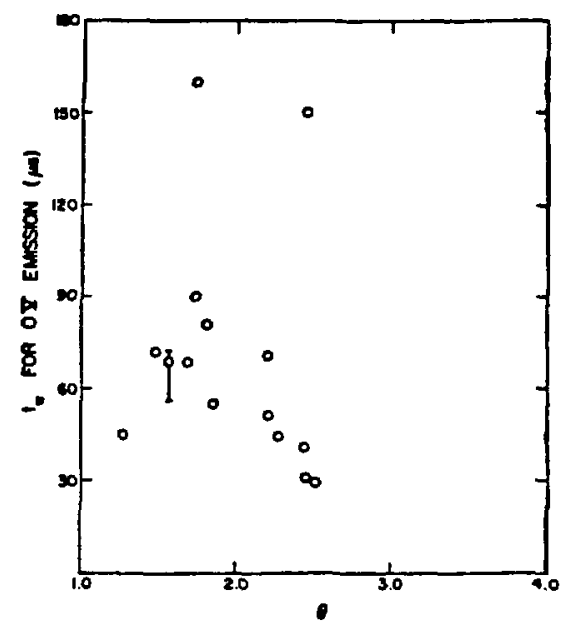

Fig. XVII-10.

Full width time $t_{w}$ for $0 \mathrm{~V}$ ine emission at a filling pressure of 20 mtorr.

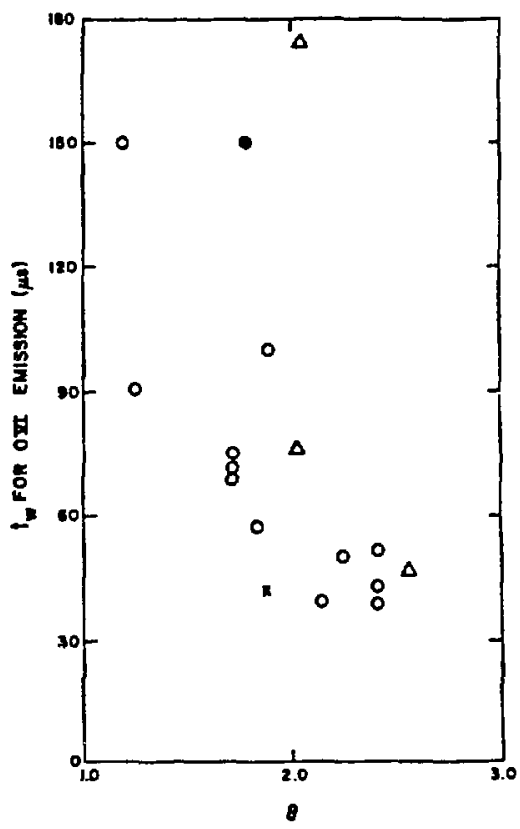

Fig. XVII-11.

Full width time $t_{w}$ vs $\theta_{\mathrm{m}}$ for $o v I$, o - 20 mtorr, - 10 mtorr, $x-6$ mtorr, $\Delta-2$ mtorr.

signal should also scale with $\theta_{\mathrm{m}}$. The experimental evidence for this is shown In FIg. XVII-12.

B. Plasma Confinement

Insufficient data exist in the present mode of operation to determine energy confinement time. However, a measure of the duration of the discharge is the decay time for $I_{\phi}$. This decay time increases with increasing $\theta_{m}$ for $\theta_{\mathrm{m}}^{\prime} \mathrm{s}>1.5$, as shown in Fig. XVII-13. The decay time has a dependance which is proportional to the plasma inductance divided by resistance. The resistance is determined by a combination of the crowbar gap resistance and the plasma resistance. Because the crowbar gap resistance is large, $260 \mu \Omega$ at $300 \mathrm{kA}$ for example, the decay time is not very sensitive to decreasing plasma resistance.

Another measure of the duration of the discharge can be the time for reappearance of $O V I$. The time from the first $O$ VI peak to the second $O$ VI peak is plotted vs $\theta_{\mathrm{m}}$ in Fig. XVII-14. These data indicate the plasma discharge time increases as $\theta_{m}$ increases from 1.5 to 2.5 . The time between 


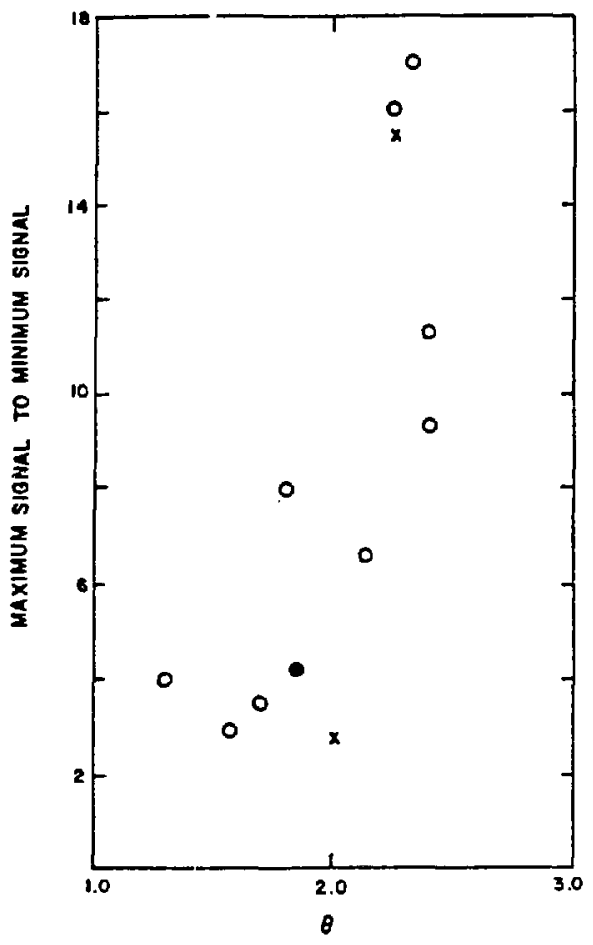

F1g. XVII-12.

Ratio of maximum signal to minimum signal. 0 - 20 mtorr, $\bullet-6$ mtorr, $x-2$ mtorr.

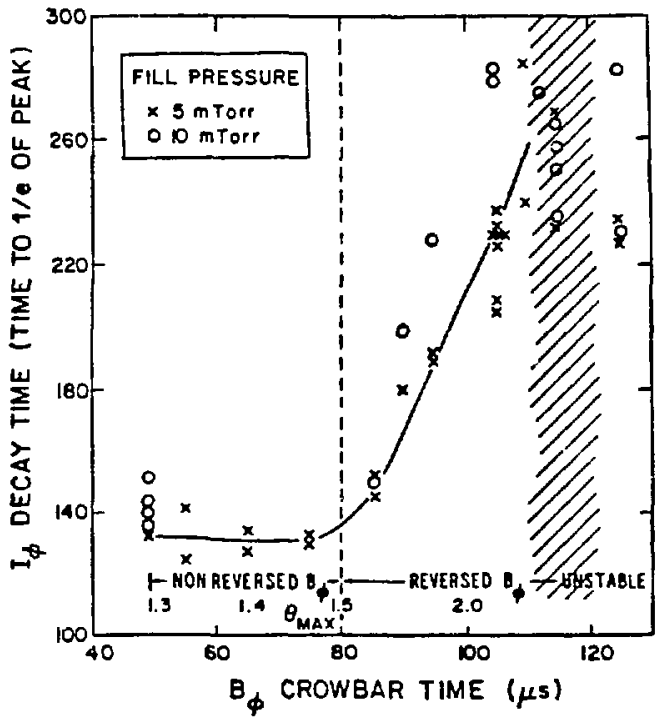

F1g. XVII-13.

Current decay time for $I_{\phi}$ vs crowbar time on $B_{\phi}$ or alternatively $\theta_{\mathrm{m}}$.

0 VI peaks is also plotted vs the $I_{\phi}$ decay time in Fig. XVII-15. This itself indicates the time between peaks is a measure of the plasma ilfetime.

C. Temperature vs Density

A pressure scan was made to determine the temperature vs density from the full width time $t_{w}$. The scaling of $t_{w}$ for 0 VI vs fill pressure is shown in Fig. XVII-16. The electron density obtained vs fill pressure is shown in Fig. XVII-17. From this it appears that full lonization is only obtained below 6 mtorr. Using the particular density data for the shot being analyzed, the full width $t_{w}$ is plotted vs electron density (during the 0 vI signal) in Fig. XVII-18. The solid lines on this graph represent temperature 1sotherms from FIg. XVII-3. Temperatures of about 50-60 eV appear at the highest densities whereas the densities below $6 \times 10^{14} \mathrm{~cm}^{-3}$ have temperatures of approximately $100 \mathrm{eV}$. 


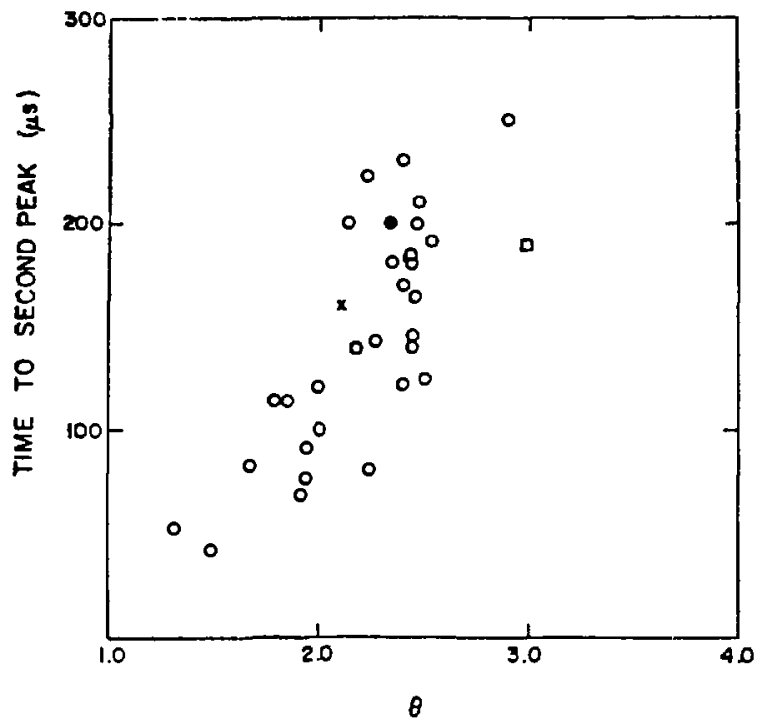

Fig. XVII-14.

Time $t_{B}$ between successive peaks of the observed line emission of either $0 \mathrm{~V}$ or 0 VI is plotted vs $\theta_{m}$.

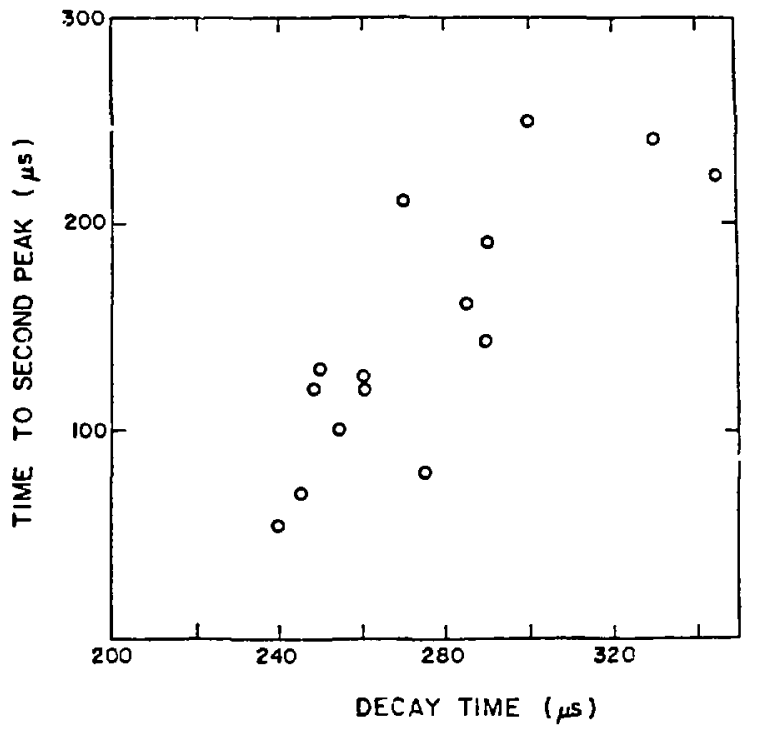

F1g. XVII-15.

Time between peaks $t_{B}$ is plotted vs decay time of the plasma current.

A pressure scan was also made for data taken at a lower current of $\sim 330 \mathrm{kA}$. The full width $t_{w}$ is plotted vs fill pressure in Fig. XVII-19. Also shown on this graph are values of $t_{w}$ vs pressure (foints), each of which correspond to the calculated temperature range shown. The temperature range of 30-55 eV seems to best characterize these experimental data. D. Description of the Computer Code 0.J.2. (Developed by Ortolani and Jahoda) The zero-dimensional code solves an intial value problem for 11 simultaneous differential equations:

$$
\begin{aligned}
& \frac{d n_{1}}{d t}=n_{1-1} s_{1-1}\left(T_{e}\right)-n_{1} s_{1}\left(T_{e}\right)-n_{1} \alpha_{1}\left(T_{e}, n_{e}\right)+n_{1+1} \alpha_{1+1}\left(T_{e}, n_{e}\right), \\
& I=1,9 \quad \mathrm{n}_{2} \text { through } \mathrm{n}_{9}=0 \text { initially, }
\end{aligned}
$$

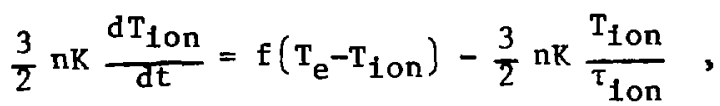




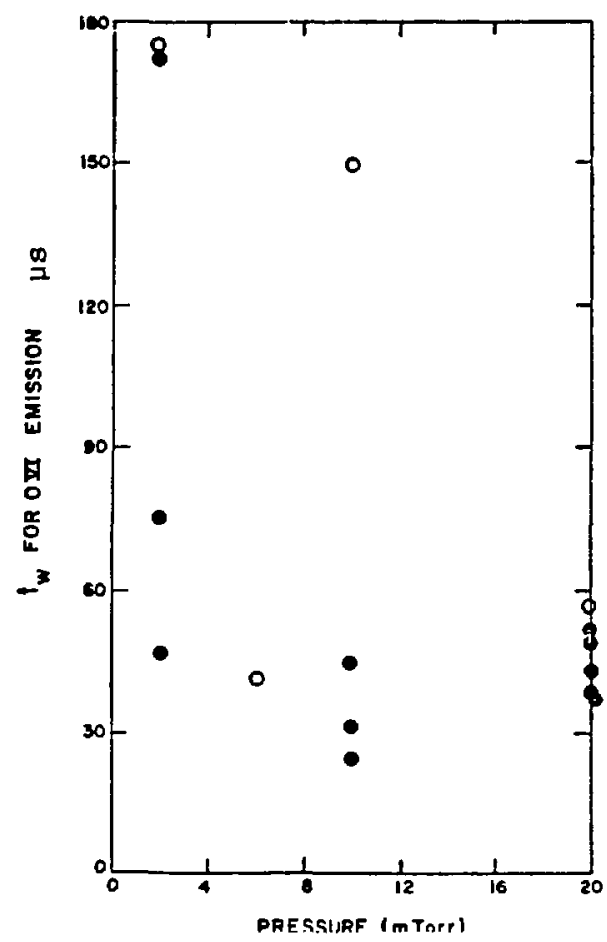

Fig. XVII-16.

Full width time $t_{w}$ vs fill pressure for $O V I, \circ-\theta_{\mathrm{mm}}=1.77-1.84$, $\theta_{\mathrm{m}}=2 \cdot 0-2 \cdot 5$.

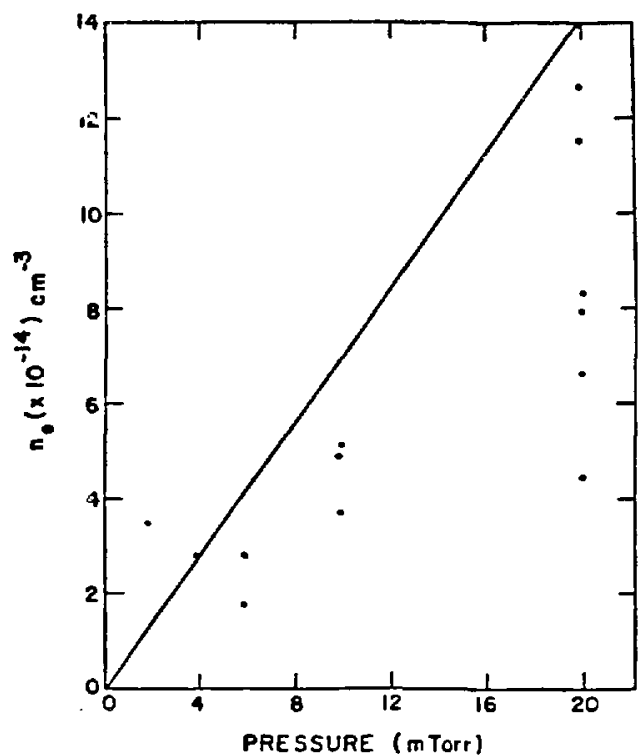

Fig. XVII-17.

Electron density vs fill pressure. The solid line represents $100 \%$ ionization.

$\frac{3}{2} \mathrm{nK} \frac{\mathrm{dT}}{\mathrm{dt}}=$ ohmic heating $-\mathrm{f}\left(\mathrm{T}_{\mathrm{e}}-\mathrm{T}_{\text {ion }}\right)-$ line radiation -

$$
\text { continum radiation }-\frac{3}{2} \mathrm{nK} \frac{\mathrm{T}_{\mathrm{e}}}{\mathrm{\tau}_{\mathrm{e}}} \text {, }
$$

$\sum_{1}^{9} n_{1}=n_{\text {oxygen }}$,

$\mathrm{n}=$ electron density,

$n_{\text {oxygen }}=$ total oxygen impurity density , 


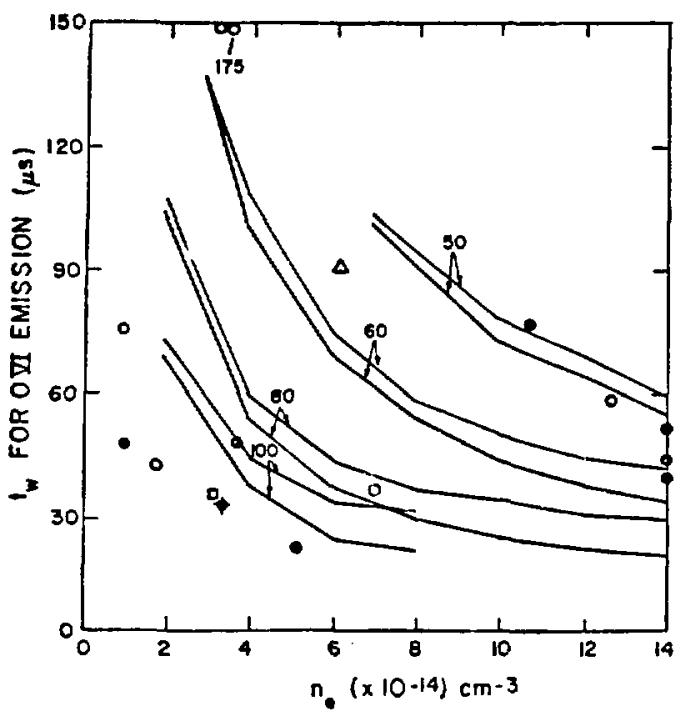

Fig. XVII-18.

Full width time $t_{w}$ vs electron density. The solid lines represent temperature isotherms for ramp times of 25 and $50 \mathrm{\mu s,}$ with the temperature maximum given for each pair.

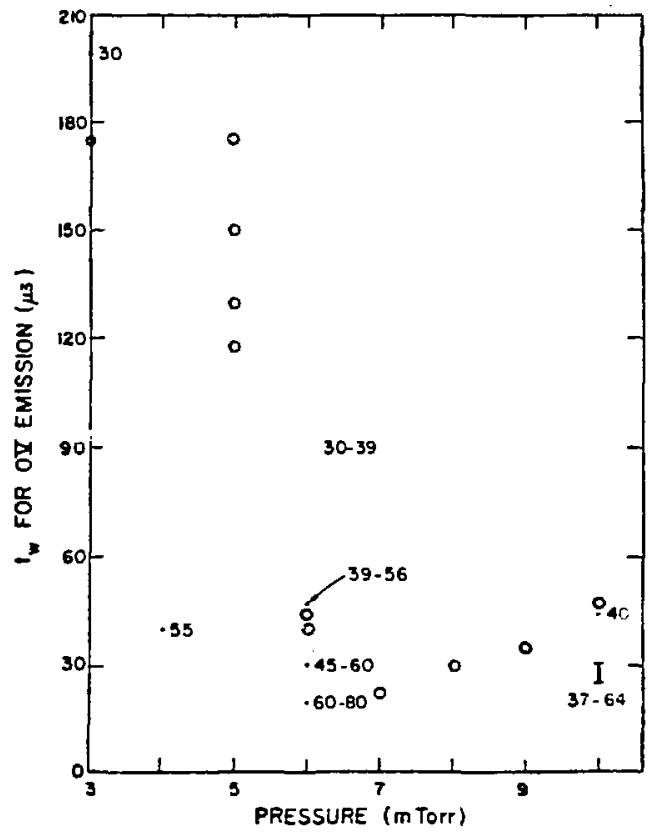

Fig. XVII-19.

Full width time $t_{w}$ for $0 v$ emission. These dala come from 330-kA conditions.

where $f\left(T_{e}-T_{10 n}\right)$ is the electron ion equilibration term, and $S_{1}\left(T_{e}\right)$ and $\alpha_{1}\left(T_{e}, n_{e}\right)$ are lonization and recombination coefficlents, respectively.

In addition, a circuit was coupled to the plasma model, with the current density being determined by the circuit and a form factor.

E. Conditions Needed For Observed 0 VI Behavior

The time behavior of $O V$ and $O V I$ were used as a comparison between the $0 . J .2$ code results and the experiment. In the ohmic heating term of the electron energy equation, there is a form factor used to account for a current distribution being present. By increasing this form factor another factor of two, the plasma heating could be obtained which was necessary for the observed t1me development of $\mathrm{OV}$ and $\mathrm{O} \mathrm{VI}$. The temperature and toroldal plasma current are shown in Fig. XVII-20 for conditions of aided reversal and a peak current of $500 \mathrm{kA}$. 


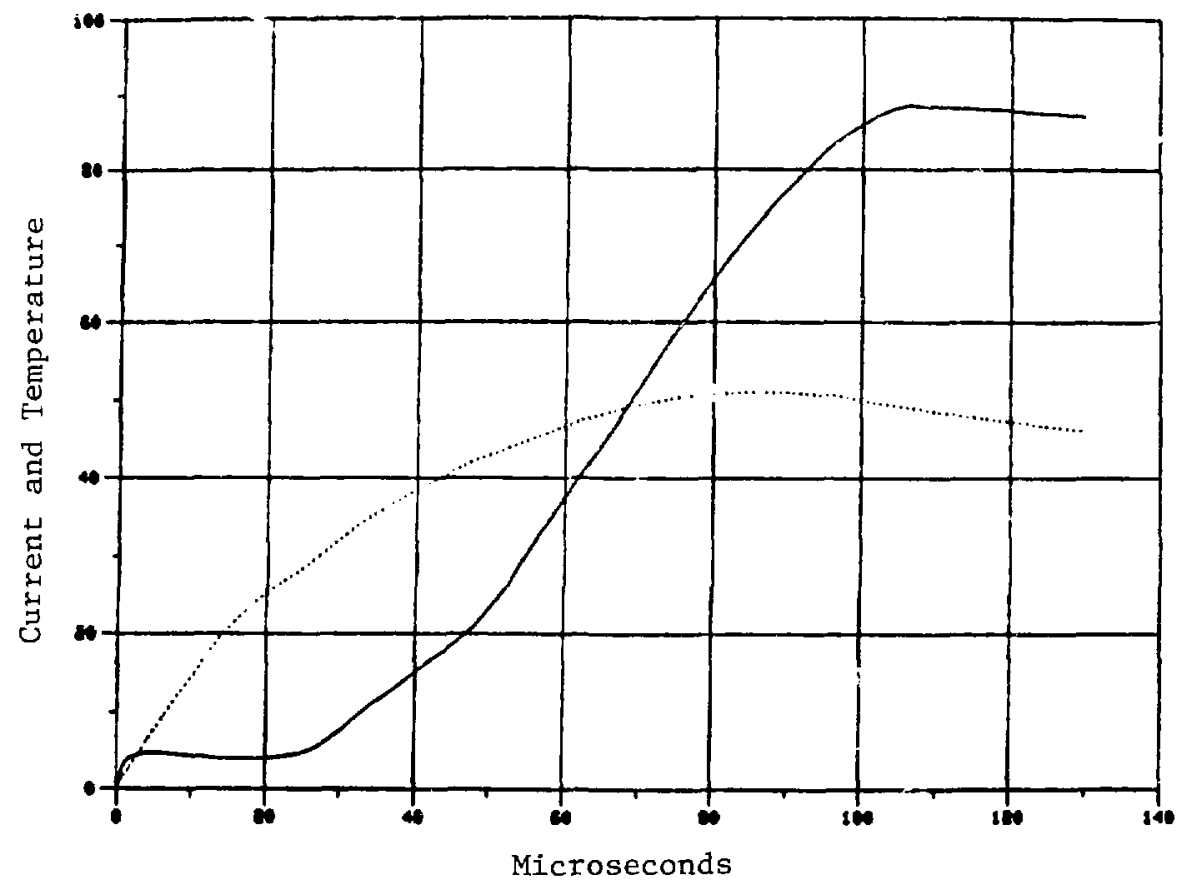

Fig. XVII-20.

Dotted line is toroidal current in kiloamps. Solid Iine is electron temperature in electron volts.

\section{SUMMARY}

The emission from oxygen impurity lines in the $Z \mathbf{T}-40$ plasma was used to determine the electron temperature. The emission data was quantified by the time of burnthrough $t_{w}$, which was then plotted vs $\theta_{m}$ and density. The temperature necessary for the observed $t_{w}$, assuming 1deal conditions, 1s found using the modified 0.J.l code. In the 500-kA case, with aided reversal of $B_{\Phi}$, temperatures of about 50-60 eV are necessary to explain the time development of $O \mathrm{~V}$ and $\mathrm{O} V \mathrm{I}$ at the filling pressures of $20 \mathrm{mtorr}$, whereas for filling pressures below 10 mtor $:$, temperatures of approximately $100 \mathrm{eV}$ are required.

The decay time of the current is increased by the increase of $\theta_{\mathrm{m}}$ above 1.5. This is consistent with an increasing plasma lifetime and is supported by the time of reappearance of $O \mathrm{VI}$. 


\section{ACKNOWLEDGMENTS}

People who have contributed to the nonspectroscopic diagnostic measurements or analyses mentloned here are Don Baker, Abe Jacobson, Robert Wat $t$, Ke1th Thompson, and Jerry Buchenauer. Watt, Thompson, and Jim Downing helped in some of the spectroscopic measurements. Ken Klare provided the computer software for the on-line data analysis. Armando Rendon helped keep the vUV equipment in operation.

\section{REFERENCES}

1. S. Ortolani, "A Zero Dimensional Plasma Model and the Study of the Initial Performance of the ZT-40 Experiment," Los Alamos Sclentific Laboratory report LA-8261-MS (March 1980).

2. A. C. Kolb, R. W. P. McWhirter, Phys. Fluids, 7, No. 4, P. 519 (1964).

3. S. Ortolani (ref. 1), Appendix.

4. Emory Stova1, FR 80 Library of Plotting Subroutines for the PDP-10, Los Alamos National Laboratory Group CTR-10 1nternal report (December 1979).

5. A. R. Jacobson, Rev. Sc1, Instrum. 49, No. 5 (May 1978). 
CHAPTER XVIII

ELECTRON TEMPERATURE ESTIMATES FROM HELIIM-LIKE ION LINE EMISSION

by

P. G. Weber

\section{INTRODUCTION}

It is generally agreed that Thomson scattering of laser light provides a definitive measurement of the electron velocity distribution, and hence the plasma temperature. Its major disadvantages are that either only a few points in time are avallable at a specific scattering point (using a multiple-pulse laser), or a number of scattering points can be examined at one instant of time. Both a multiple-pulse laser system and "two-dimensional" Thomson scattering are being implemented on 2T-40. Spectroscopic measurements can also be used to derive a temperature. These methods allow a continuous estimate of temperature, but are less accurate than scattering and less precise in spatial location. I describe such a method here, based on the emission of Helfum-1ike impurity ion radiation which was recently implemented on $\mathrm{ZT}-40$.

\section{THEORY}

In simplest terms, the Hellum-1lke ion may be considered as a three-level structure, with plasma excitation from the ground state to the $2^{3} \mathrm{p}$ upper leve1, followed by radiative decay to the $2^{3} \mathrm{~S}$ state. If we assume that excitation to the $2^{3} \mathrm{P}$ state is only due to plasma effects, and that all of the de-excitation is by radiative transaction, then, for a Maxwellian energy distribution we may write ${ }^{l}$ 


$$
I=2\left(\frac{2 k T_{e}}{\mathrm{~m} \pi}\right)^{1 / 2} \mathrm{~N}_{1} \mathrm{n}_{\mathrm{e}} O \mathrm{~h} v\left(1+\frac{\mathrm{E}_{12}}{\mathrm{kT}}\right) \exp \left(\frac{\mathrm{E}_{12}}{\mathrm{kT} \mathrm{T}_{e}}\right) \text {, }
$$

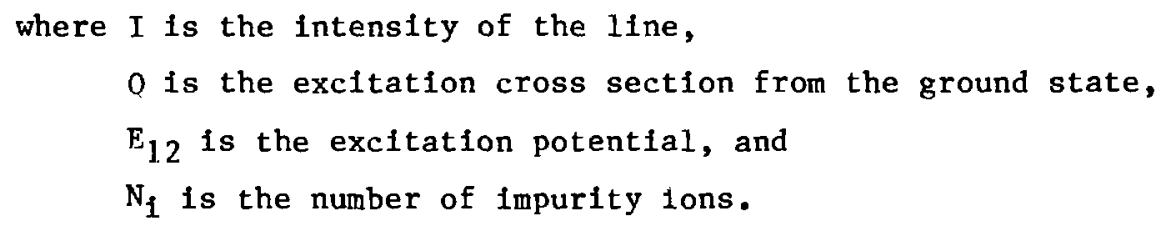

Evaluation of this expression using the appropriate cross sections ${ }^{2}$ shows a very strong dependence of the line intensity on the electron temperature for the species considered, namely, $C V$ at $2271 \AA, E_{12}=304 \mathrm{eV}, \mathrm{N}$ VI at $1897 \AA$, $E_{12}=426 \mathrm{eV}$, and $O$ VII at $1624 \AA, E_{12}=568 \mathrm{eV}$. Thus by measuring the line intensity, electron density, and estimating the Ion population, we may obtain a reasonable estimate of the temperature.

It is also noted that the threshold temperature for observation of a line varies from $\sim 30 \mathrm{eV}$ for $\mathrm{CV}$ to $70 \mathrm{eV}$ for 0 VII. This suggests an alternative temperature estimate may be obtalned by taking the ratio of intensities of two Ines,

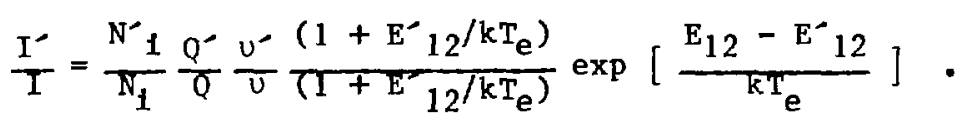

Thus by measuring the relative strength of two Helium-1ike impurity lines, and estimating the ratio of their concentrations, we may a1so arrive at a value of electron temperature. Again, the ratio of intensities is a strong function of temperature, so that great measuring accuracy is not required to obtain reasonable temperature estimates.

It should be noted that for Helium-like lons, the lowest excitation energies are much higher than the ionization energy of the preceeding Helium-1ike Ions (e.g. for $C V, E_{12}=304 \mathrm{eV}$; for $C I V, E_{10 n}=65 \mathrm{eV}$ ). Thus we are justified in neglecting production of ground-state ions during the time that line emission is observed. There is also no time dependence in the model, so that in a transient plasma we may see lonization states at a time 
when the extant temperature would indicate higher states. Thus we may underestimate temperatures early in time using this diagnotic.

The simple model described above has been 1mproved by inclusion of losses due to Ionfzation from the upper level and transfer to $n=2$ singlet levels ${ }^{3}$. Curves for C V $(2271 \AA)$ and 0 VII $(1624 \AA)$ for densities less than $10^{15} \mathrm{~cm}^{-3}$ are presented in Fig. XVIII-1.

\section{EXPER IMENT}

As a first step to implementing this diagnostic, CV (2271 $\AA)$ radiation from ZT-40 was examined. Light was gathered from a vertical chord and dispersed by a McPherson Mode1 216 one-meter spectrometer having a dispersion of $7 \AA / \mathrm{mm}$. The instrument was calibrated using a standard tungsten ribbon lamp and a calibrated mercury spectral lamp. The existence of the $C V$ triplet was confirmed, as shown in FIg. XVIII-2, where the intensity observed, normalized to the electron density, is plotted as a function of wavelength. The individual components are not resolved due to the $4 \AA$ FWHM resolution of

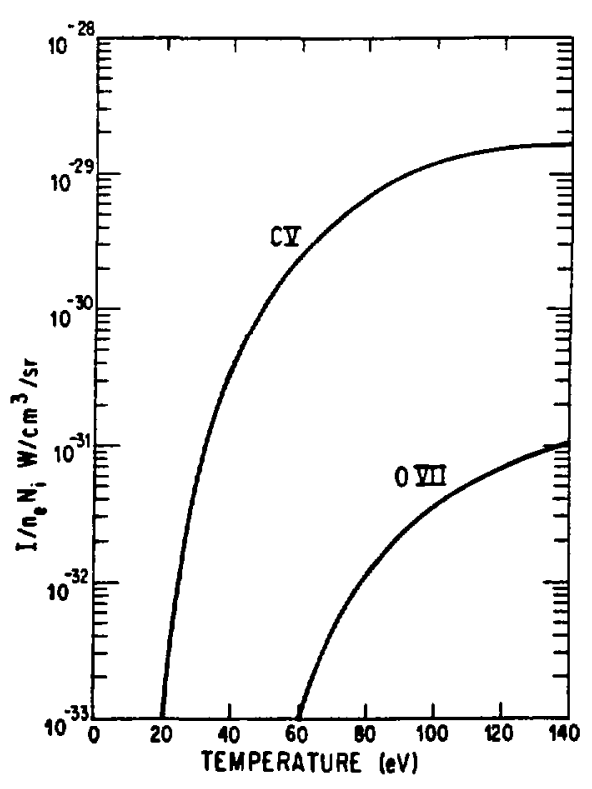

F1g. XVIII-1.

Line intensity as a function of electron temperature.

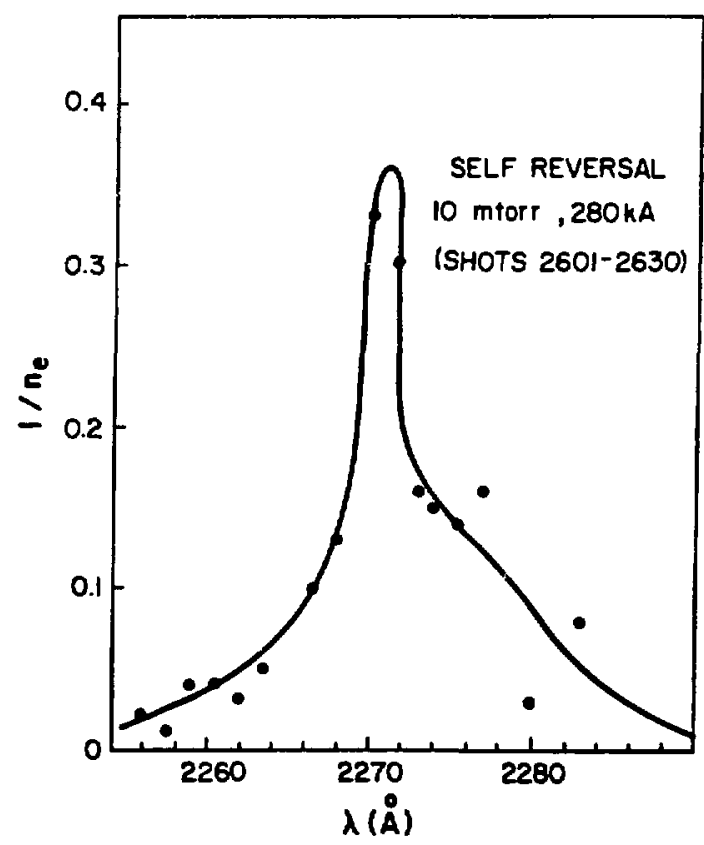

F1g. XVIII-2.

Intensity (normalized to electron density) at vicinity of $C v$ triplet. 
the system. An estimate of the impurity ion population was then obtained by comparing the $\mathrm{C} \mathrm{V}$ line emission to the single-point Thomson scattering temperature for a wide variety of conditions, primarily at later times ( $~ 300 \mu \mathrm{s}$ ) when equilibrium might reasonably be expected to have been achieved. The average value of this number of radiators was then taken as fixed for the remaining experiments. Note that a factor of 2 error in this estimate would typically affect the derived temperature by $\pm 4 \mathrm{eV}$.

Figure XVIII-3 shows the temperature history for four shots (each symbo1 pertains to a particular shot). The shots are seen to be reasonably reproducible, and temperatures above the "Oxygen Radiation Barrier" are maintained. Agreement with Thomson scattering data at later times is good, but Thomson scattering shows temperatures near $100 \mathrm{eV}$ near peak current $(\sim 150 \mu \mathrm{s})$. Our underestimate using the present diagnostic is attributed to insufficient time being avallable to reach an equilibrium, as well as the possibly peaked temperature profile at this time leading to higher temper:tures on axis (Thomson) than averaged over a finite volume (as in the present method).

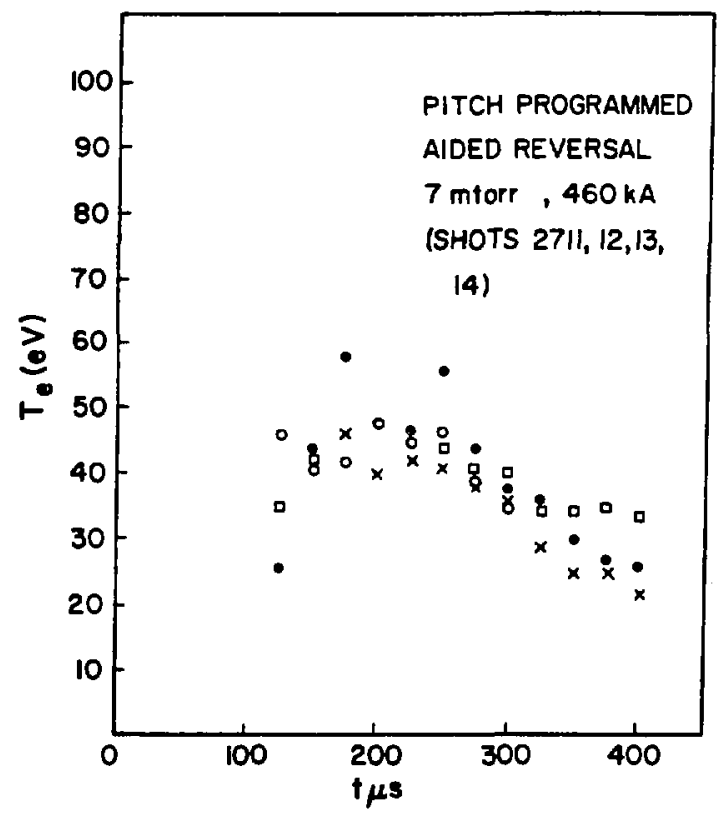

Fig. XVIII-3.

Electron temperature as a function of time as from $C V$ intensity. 
IV. CONCLUSTON

The observation of Helium-like impurity lines allows a reasonably accurate estimate of electron temperature to be made as a function of time in 2T-40. The data compares favorably to Thomson scattering temperatures, and provides a valuable continuous monitor on electron temperatures. The experiment is being extended to observe two spectral lines simultaneously along the same chord, allowing temperature estimates to be made from intensity ratios rather than the absolute measurements.

\section{REFERENCES}

1. R. V. Williams and S. Kaufman, Proc. Phys. Soc. LXXV, 329 (1959).

2. N. H. Magee, Jr., J. B. Mann, A. L. Merts, and W. D. Robb, "Electron Impact Excitation of Carbon and Oxygen Ions," Los Alamos Scientific Laboratory report LA-6691-MS (April 1977).

3. H.-J. Kunze, A. H. Gabriel, and H. R. Griem, Phys. Fluids 11, 662 (1968) 
CHAPTER XIX

RADIALLY RESOLVED LUMINOSITY MEASUREMENTS

by

R. G. Watt

A simple but very versatile system for observing the radlal distribution of radiation in the visible portion of the spectrum has been in use on ZT-40 since about shot 1200. The system consists of five fiber-optic 1ight guides, transmission filter, and photomultiplier (PM) tube assemblies. The light from a particular chord (defined by the honeycomb sections of the primary) is focused on the end of a crofon light pipe, which transmits light in the 4400-7000 \& region. The light is subsequently passed through a band pass transmission filter and detected by a type-1P21 PM tube. At present, two filters have been used, one centered at $486.1 \pm \sim 5 \mathrm{~nm}\left(D_{B} 1\right.$ ine) and one centered at $520 \pm 4.3 \mathrm{~nm}$. This second filter passes a wavelength at which no Iines have ever been observed on any photographic plots taken of ZT-40. Examination of wavelength tables ${ }^{l}$ show no known lines from impurities present in $2 \mathrm{~T}-40$ in this region. This system has also been used with no filter but the results are indistinguishable from those using the $D_{\beta}$ filter.

The comparison of the $D_{\beta}$ and $520 \mathrm{~nm}$ results for a particular type of shot indicate that most of the early ( $<50 \mu$ s after current initiation) radiation in the visible is due to $D_{B}$. Radiation coming from the plasma after that is a mixture of $D_{\beta}$ and bremsstrahlung (or Iine radiation; about $1: 1$ in the 10-nmwide filter window). Figure $X I X-1$ shows the radiation in the $D_{\beta}$ region as a function of time for a stablilzed pinch discharge at 7 mtorr, with $\theta \sim 1.5$ and peak current of approximately $430 \mathrm{kA}$. Figure XIX-2 shows the radiation seen through the 520-nm filter for an Identical shot. Figures XIX-3 and XIX-4 show the radial dependence of the $D_{\beta}$ and $520-n m$ radiation via a three-dimensional plot. 

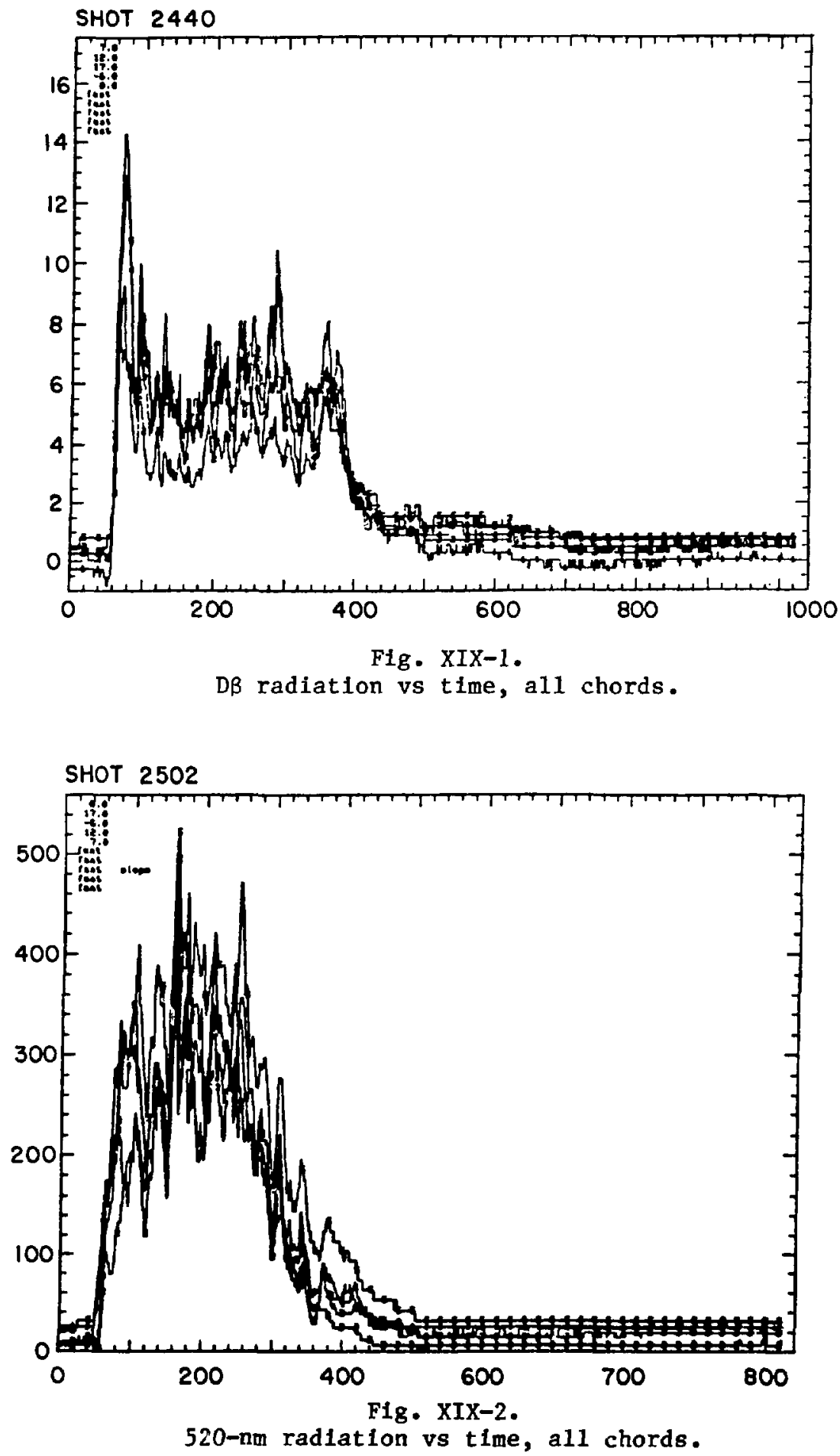


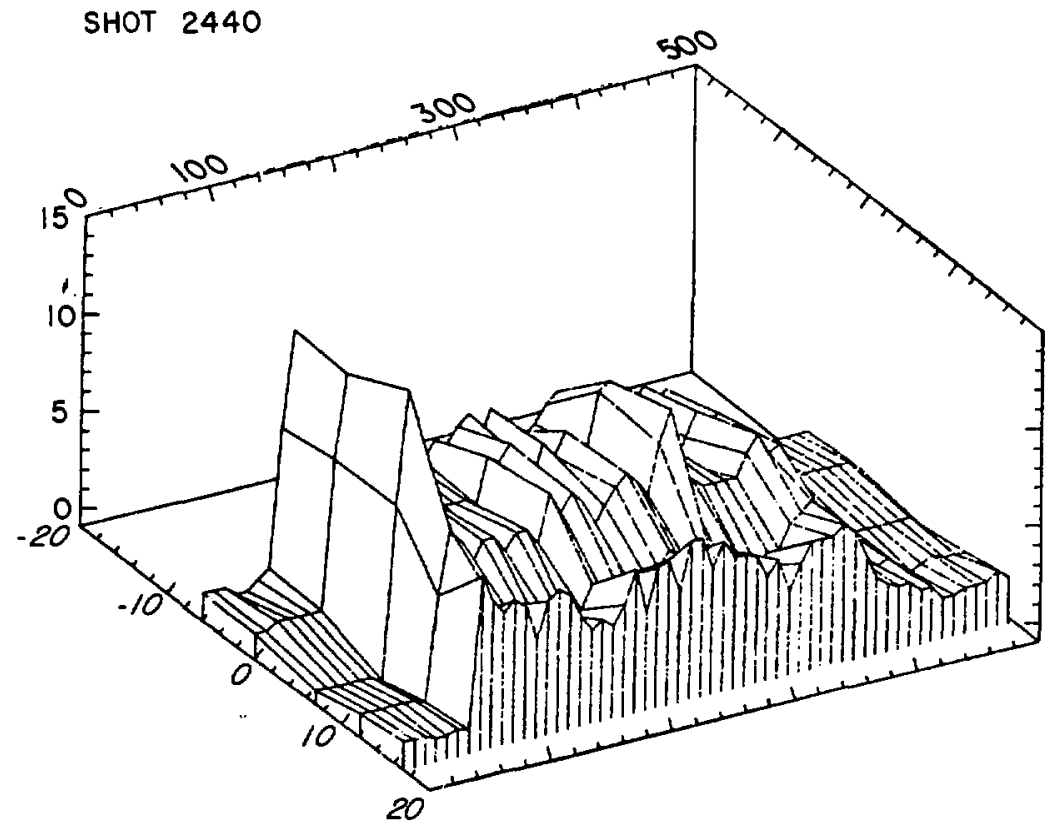

Fig. XIX-3.

DB radiation profiles vs time.

SHOT 2502

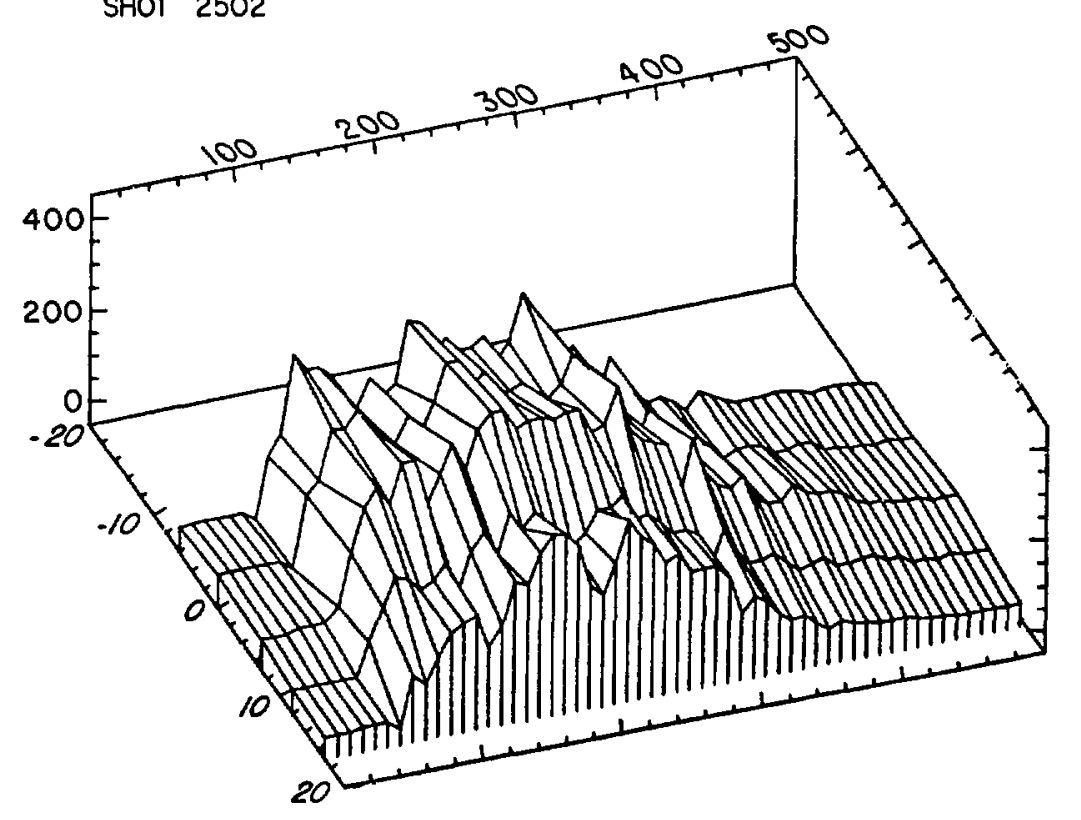

Fig. XIX-4.

$520-\mathrm{nm}$ radiation vs time. 
The radiation in the 520-nm window is much gentler in its time dependence than the $D_{B}$ radiation and is slightly (-20-30\%) peaked on axis. The $D_{\beta}$ radiation comes up rapidly during current initiation, then falls to some ldilng level that is a combination of $D_{\beta}$ and the surrounding bremsstrahlung (which makes the $D_{B}$ radial profile difficult to interpret) and then cuts off abruptly. This latter phenomena is probably caused by the $D_{\beta}$ radiation having turned back on while the background was dying away and then turning off rapidly as the plasma temperature collapsed below the $D_{B}$ norm temperature ${ }^{2}$ late in the discharge $\left(T_{e}\right.$, norm $\left.\sim 8 \mathrm{eV}\right)$.

The behavior of the $D_{\beta}$, while interesting in 1 ts own right, can only be used to calculate $\mathrm{T}_{\mathrm{H}}$, after correcting for the electron density and making some assumption about the profile of neutrals in the plasma. The assumptions make interpretation difficult. The bremsstrahlung, however, can in principal $\therefore e$ used to calculate $T_{e}$, after correcting for $n_{e}(r)$ and assuming something $x$ at the ion distribution $n_{1}(r)$. A reasonable assumption might be that $n_{+}(r) \sim n_{e}(r)$. In either case the interpretation demands an absolute calibration that is difficult to obtain, and in the case of bremsstrahlung, some knowledge of $z_{\text {eff }}$ is required. In many cases this method has been used to calculate $z_{\text {eff }}$ and this may be done here in the future. A more useful approach is $\ldots$ "callbrate" the bremsstrahlung on a given shot by comparing the Thomson scattering temperature to the bremsstrahlung and then using the time dependence of the bremsstrahlung to gain some insight into the time history of the discharge. To do so one must make some assumption about $z_{\text {eff }}$, the simplest being that $z_{\text {eff }} \sim$ constant during the discharge. Since this is only to be used as an indicator of the time history of the temperature, this is probably not too bad an assumption. Unfortunately, since the radiated power has a dependence

$$
\frac{d p}{d \nu} \propto \frac{n_{e}^{2} z_{e f f}^{2}}{T_{e}^{1 / 2}} e^{-h \nu / k T_{e}}
$$

and the filter is such that $h \nu \ll k \mathrm{kT}_{e}$, the dominant dependence is on the $\mathrm{n}_{e}^{2}$ term. Considering the large fluctuation level on the $f n_{e} d \ell$ signals from the interferometer, the resultant temperature profiles are at best merely 
qualitative. Seen in this 1ight, however, they provide an ald to identifying good or bad discharges and are useful in that role. A software package is being implemented to try to eliminate the density dependence and provide an on-1ine temperature profile for use by the physicist in charge of operating the machine.

\section{REFERENCES}

1. Striganov and Svontitski1, Tables of Spectral Lines of Neutra1 and Ionized Atoms, (Plenum Data Corporation, New York, 1968).

2. Lochte-Holtgreven, Plasma Dlagnost1cs, (North-Holland Publishing Company, Amsterdam, 1968), p. 178. 
CHAPTER XX

SIMULATIONS OF ZT-40 CURRENT AND VOLTAGE WAVEFORMS

by

R. F. Gribble

The englneering coupling model for RFP devices devised by Culham appears to represent ZT-40 waveforms rather we11 and may help in understanding some of the details of ZT-40 operation. This is rather surprising since the model only assumes a current distribution and a plasma resistance. The basis of the model is the assumption that plasma current risetime is much longer than thc relaxation time to minimum energy states, so that the plasma is essentially continuously described by some approximation to such a "continum" of states. A comparison between model calculations and ZT-40 data for several modes of operation, including that of the single feedplate connection, is presented.

The Culham coupled model was devised originally to aid in the engineering design of the proposed Culham experiment, RFX. Jim Grey and Mike Bevir first developed the "Cunstant $K$ " model based on the minimum energy state theory of Taylor. Allan Newton and Wallace Johnson concelved a more general model based on total magnetic field energy. Both assume a plasma current distribution, but Newton and Johnson modified the pure Bessel function distribution so that current at the wall is zero. The modified Bessel function distribution is the solution of

$$
\nabla \times \underset{\sim}{\mathrm{B}}=\mu \underset{\sim}{\mathrm{B}},
$$

where $\mu$ is constant, $\mu_{k}$, for $r \leq .7 a$ and unlformly decreases from $\mu_{k}$ at $r=.7 \mathrm{a}$ to zero at $\mathbf{r}=\mathrm{a}$, where $\mathrm{a}$ is the wall radius. With this model $\mu_{k}$ is 


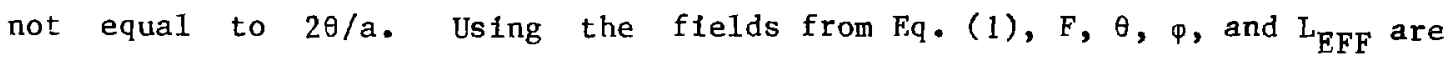
calculated where

$$
L_{E F F}=\frac{2\left(W_{m}-W_{o}\right)}{I_{z}{ }^{2}} .
$$

$W_{0}$ is the energy of the $B_{z}$ field if it were uniform,

$$
\mathrm{w}_{\mathrm{o}}=\frac{1}{2} \frac{\phi^{2}}{\mathrm{~L}_{\mathrm{o}}} \text {, }
$$

where

$$
\begin{aligned}
& L_{o}=\frac{\mu_{o} a^{2}}{2 R} \text {, and } W_{m} \text { is the total magnetic energy, } \\
& W_{m}=\int \frac{B^{2}}{2 \mu_{o}} d v \text {. }
\end{aligned}
$$

From these quantities, $F(\theta)$ and $\mathrm{L}_{\mathrm{FFF}}(\theta)$ are obtained for use in the circuit simulation. The Sceptre circuit simulation calculates

$$
\begin{aligned}
& \phi=\int \mathrm{v}_{\theta} \mathrm{dt}, \\
& \theta=\left(\frac{\mathrm{L}_{\mathrm{o}}}{\varepsilon}\right)\left(\frac{\mathrm{I}_{\mathrm{z}}}{\phi}\right), \\
& \mathrm{I}_{\theta}=\frac{\phi \mathrm{F}(\theta)}{\mathrm{i}_{\mathrm{o}}}, \\
& \mathrm{W}_{\mathrm{m}}=\int\left(\mathrm{V}_{\mathrm{z}} \mathrm{I}_{\mathrm{z}}+\mathrm{V}_{\theta} \mathrm{I}_{\theta}-\mathrm{I}_{\mathrm{z}}{ }^{2} \mathrm{R}_{\mathrm{p}}\right) \mathrm{dt},
\end{aligned}
$$

146 


$$
\begin{aligned}
& W_{o}=\left(\frac{1}{2}\right)\left(\frac{\phi^{2}}{L_{o}}\right), \text { and } \\
& I_{z}=\left[2\left(W_{m}-W_{o}\right) / L_{E F F}(\theta)\right]^{0.5} .
\end{aligned}
$$

The constant $\varepsilon$ is $a / R$ (ratio of plasma radius to major radius). Note that $W_{m}$ of Eq. (7) is calculated at each time step from the "terminals of the plasma," whereas $W_{m}$ of $E q .(2)$ is the volume integral of field energy and is a function only of $\theta$ and the field on axis for a particular assumed distribution. Since the relaxation time to the minimum energy state is assumed much less than the time scale of plasma current rise, the plasma is always in the minimum energy state given by Eq. (1), and thus the two $W_{m}^{\prime}$ 's are equal. The $I_{z}$ load consists of $R_{p}$ in series with a current source $I_{z}$, and the $I_{\theta}$ load consists only of a current source equal to $I_{\theta}$, as shown in FIg. $X X-1$. The Sceptre code does not self-consistent1y solve the circuit equations and Eqs. (4)-(9) at each time step. Some nonlinear quantities used in a time step were calculated in the previous step. By using a sufficiently small time incremert, the errors resulting from tiits approximation are usually negligible. Note that for $F=1$ the $I_{\theta}$ load is a constant" Inductance $L_{0}$, and, if $V_{\theta}$ is zero, the $I_{z}$ load is $R_{p}$

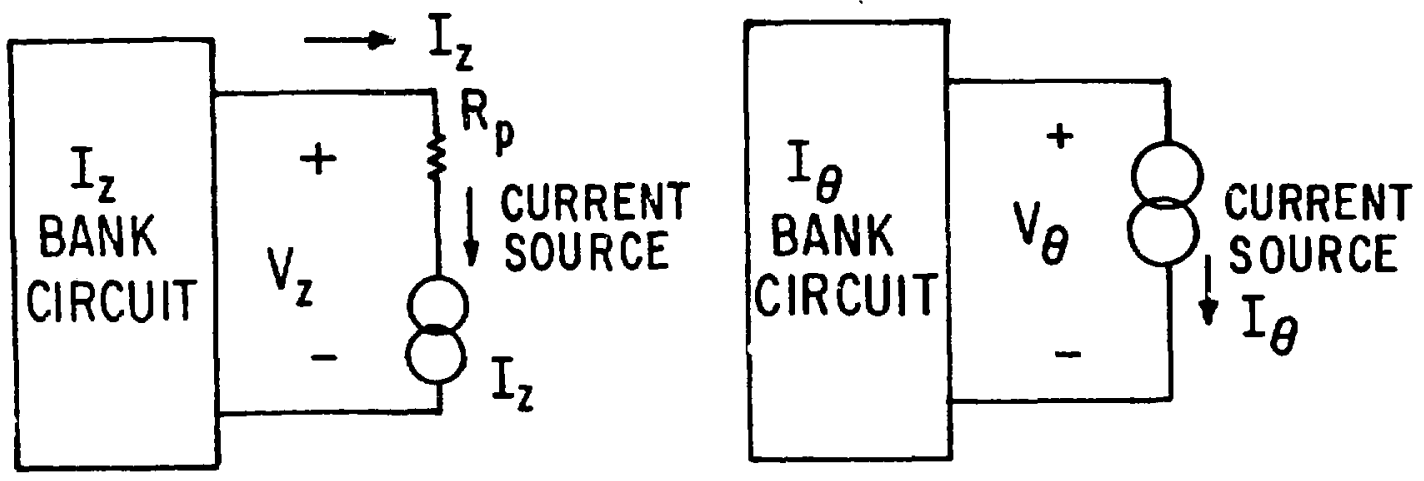

Fig. $X X-1$.

Equivalent circuit for the reversed-field-pinch coupled model. 
In serles with $\mathrm{L}_{\mathrm{EFF}}$. Voltage across $\mathrm{L}_{\mathrm{FFF}}$ is then $\mathrm{L}_{\mathrm{EFF}} \mathrm{dI} / \mathrm{dt}+\frac{1}{2} \mathrm{I}_{\mathrm{z}} \mathrm{d} \mathrm{L}_{\mathrm{EFF}} / \mathrm{dt}$. Figs. XX-2 through XX-6 show some of the simulation results. Fig. XX-2 is a comparison between the simulation and ZT-40 for alded-reversal shot 2683. Figs. $X X-3, X X-4$, and $X X-5$ are the comparisons for one-feedplate-operation shots 1281, 1282, and 1514. Figs. XX-3 and XX-5 show simulation runs for two values of plasma resistance that somewhat bracket the 7T-40 current waveform. The timing on $B_{z}$ bank voltage must be slightly in error for Figs. $X X-3$ and $X X-4$ (shots 1281,1282 ) since $B_{z}$ and flux do not quite match at the start of plasma current. From working with the model we have found that rather accurate values of $B_{z}$ clrcuit timing, $B_{z}$ circuit constants, and $B_{z}$ bank voltage are necessary for reasonable correlation of simulation with experiment. Shot 1514 was part of a set from probe measurements from which Jim Philiips calculated a plasma resistance of 0.004 ohms at peak current, a value that provides the best match for the simulation near peak current.

To observe effects on simulation of varying simulation plasma resistance, values of $0.0016,0.008$, and 0.016 ohms were used in the calculations shown in Fig. XX-6 and compared with data of shot 2683. According to the model, a factor of 10 change in plasma resistance only changes the peak current by $25 \%$, reflecting the strong influences of circult ohmic-voltage drcps on this version of the $\mathrm{ZT}-40$ circult and Indicating the reason that a constant plasma resistance provides a good fit to data.

The best fit to ZT-40 data for simulations using a constant plasma resistance occur for elther or both relatively high filling pressure and low plasma current. For these conditions, the fit is rather surprising. By employing a time-varying plasma resistance that is a function of plasma energy (with an energy decay time) and theta, better fit to higher current, lower f111 pressure data should be possible. Inttlal efforts in this direction show promise. Simulations are also being run that force the simulated plasma current to fit data by varying plasma resistance during the calculation. Such calculations may yleld a measure of plasma resistance at the "terminals" as a function of time. 
Fig. $\mathrm{XX}-2$.

Comparison of the coupled-model simulation results with ZT-40 aided-reversal shot 2683.
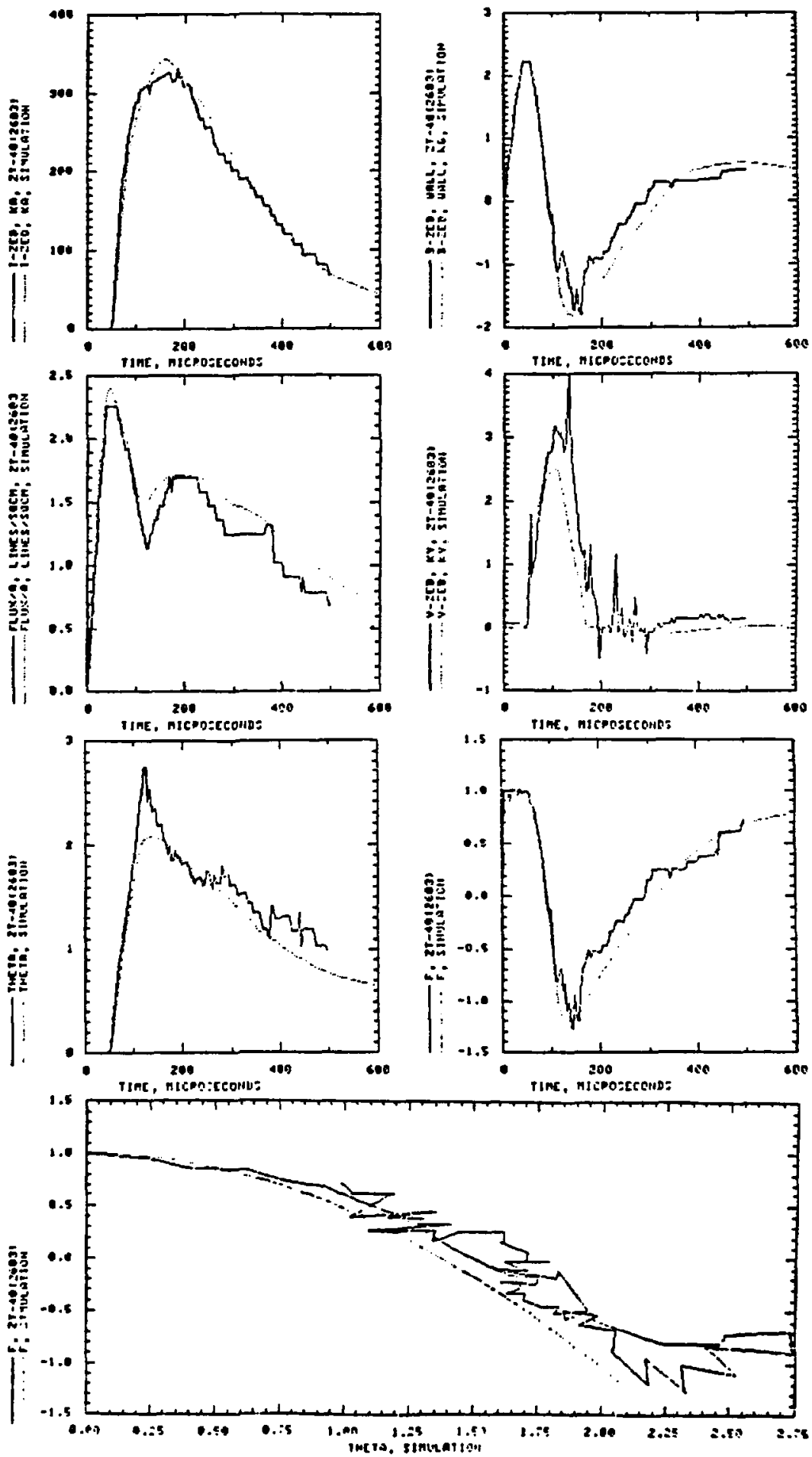

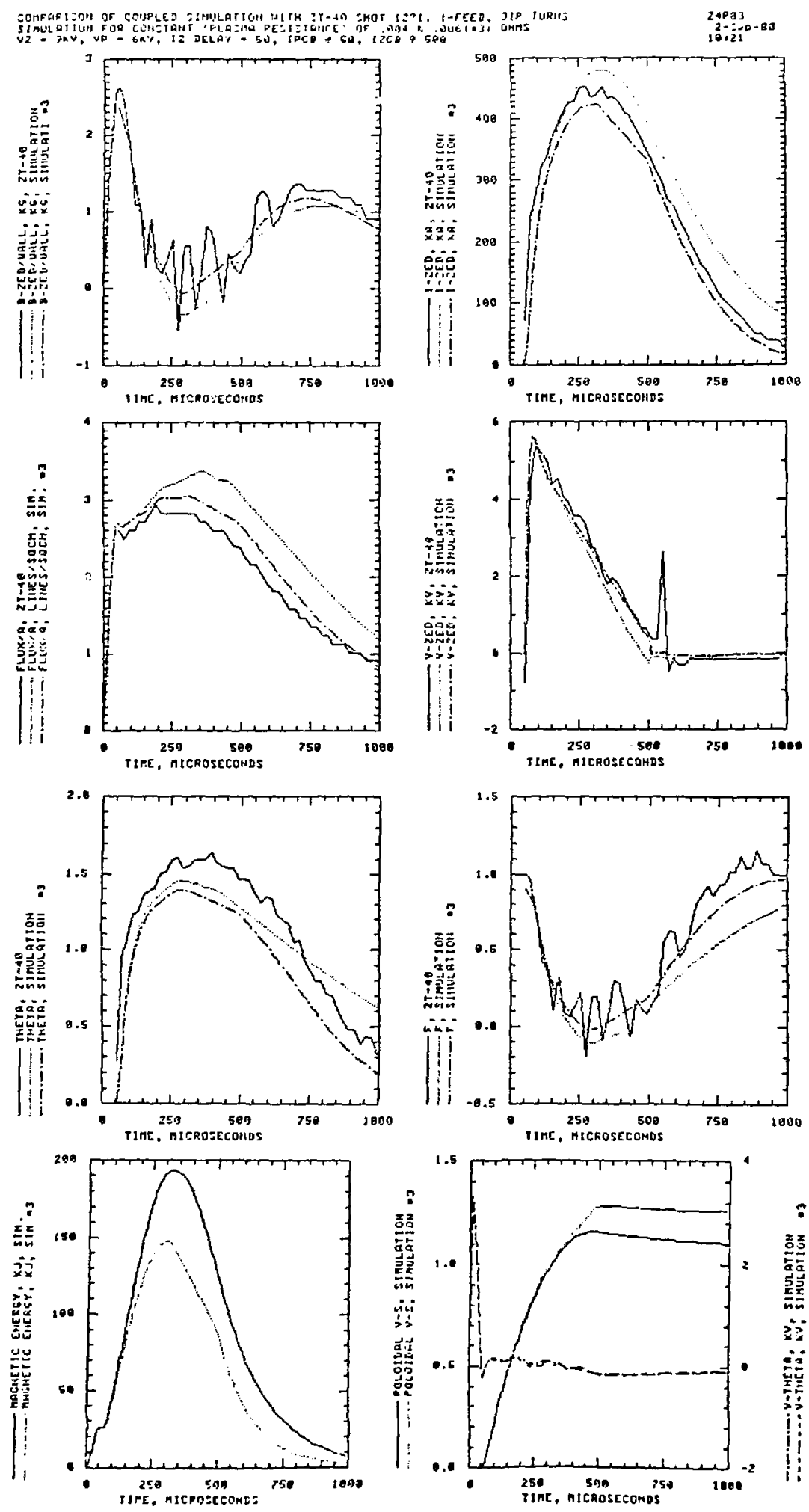

Fig. $\mathrm{Xx}-3$.

Comparison of the coupled-model simulation results with ZT-40 shot 1281 . 

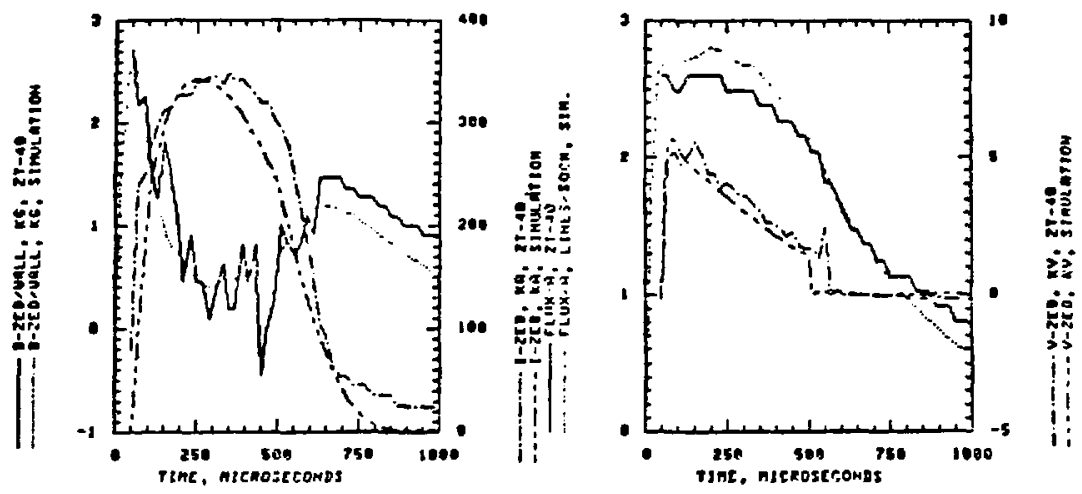

Figs. $\mathrm{XX}-4$ and $\mathrm{XX}-5$. Comparison of coupledmodel simulation results with ZT-40 shot 1282 (top) and 1514 (bottom).
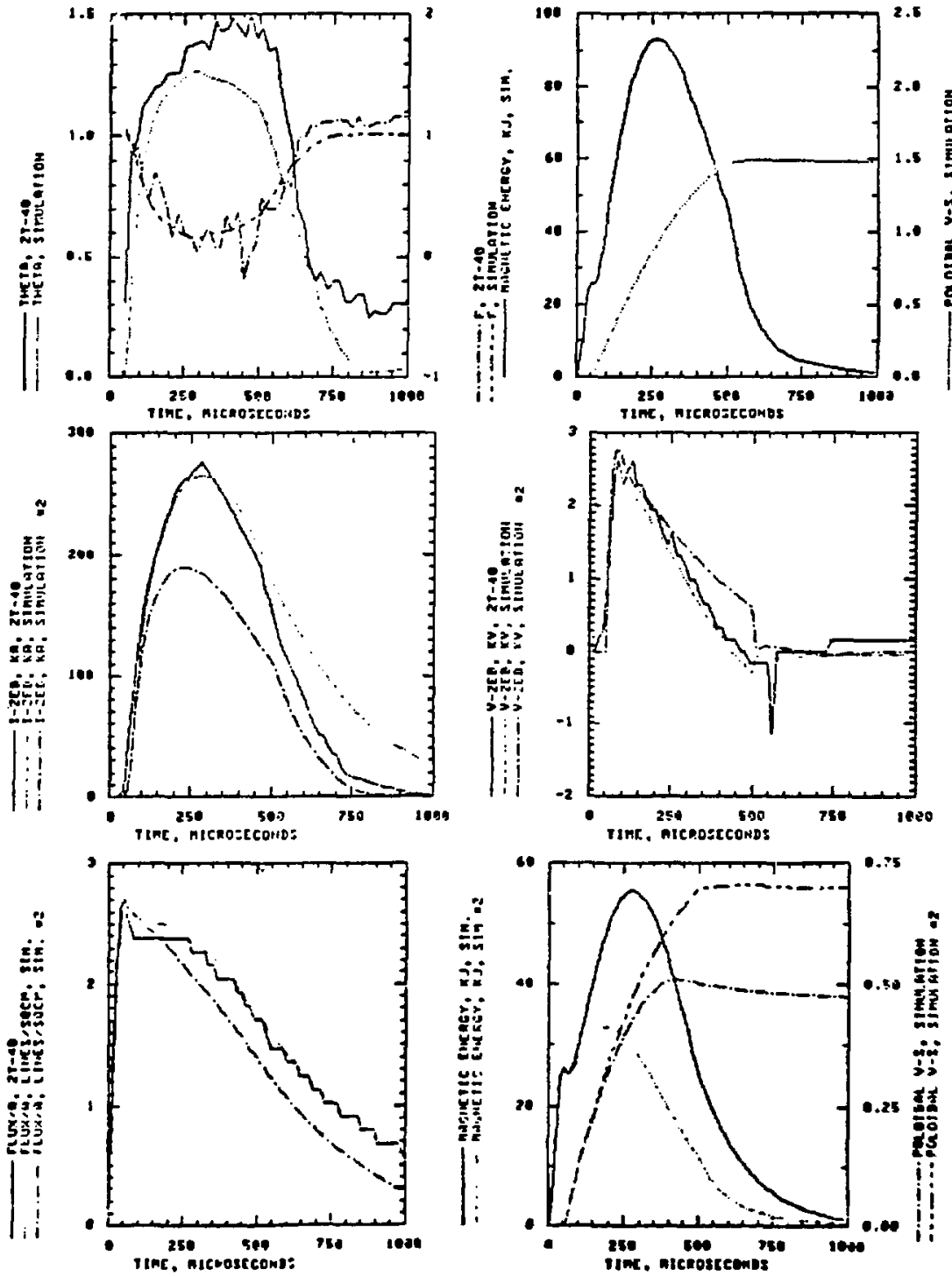

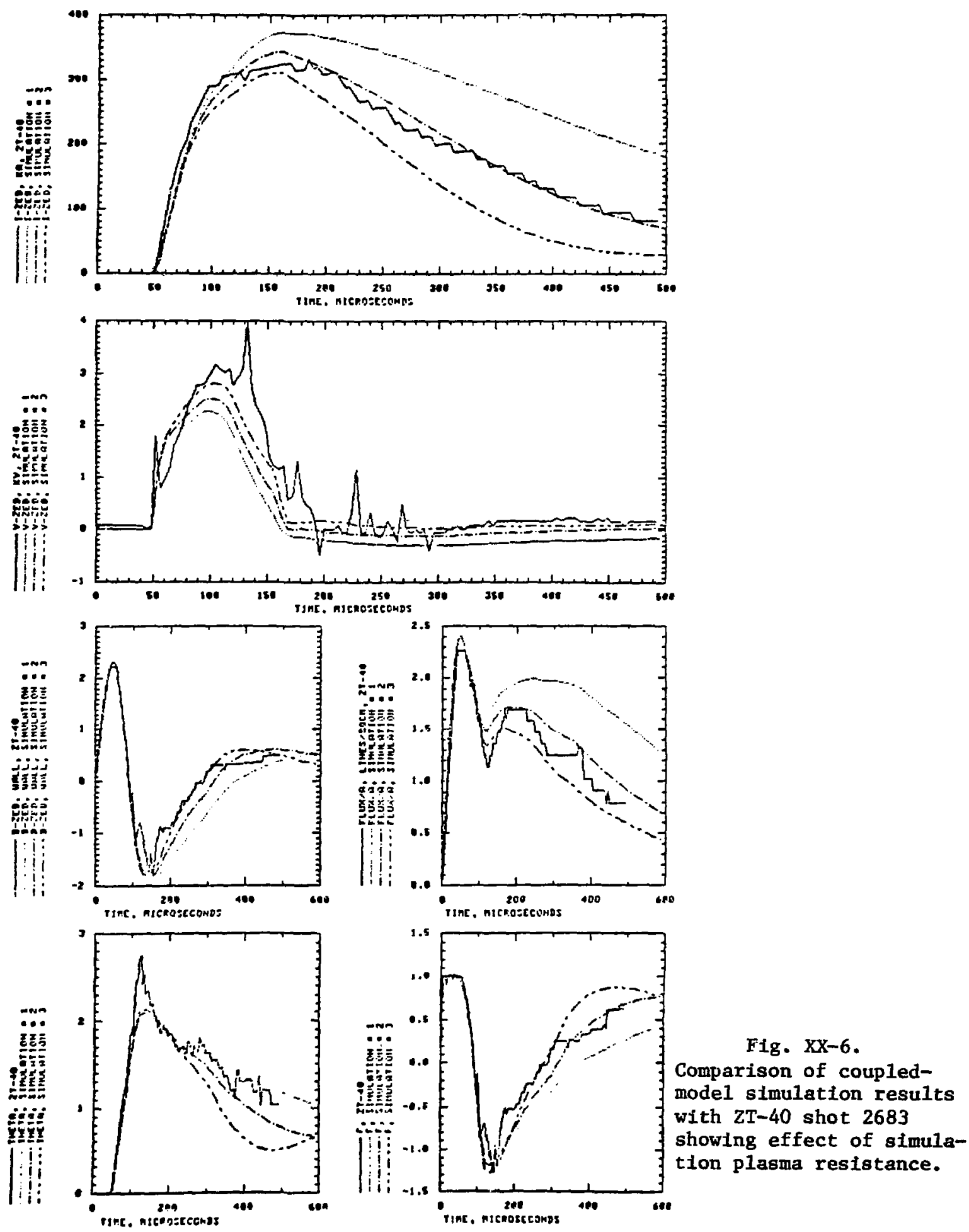


\section{ACKNOWLEDGMENT}

This work at the Los Alamos National Laboratory Involved too many of its general support groups to acknowledge individually. Specific thanks are extended to the members of the theory group CTR- 6 and computing group CTR- 10 . The authors gratefully acknowledge the administration and guidance of CTR Ttuision under Harry Dreicer and Warren Quinn. And, we further thank the $\therefore-40$ experimental team by groups, staff, and technicians:

CTR-DO

Joseph N. DiMarco

James A. Phillips

\section{CTR-2}

Don A. Baker

Guthrie Miller

Louis C. Burkhardt

Delmo D. Carney

Albert Haberstich

Laurle J. Iko

Robert B. HoweII

John L. Jo11n

John C. Ingraham

Gilbert L. Maestas

Abram R. Jacobson

Thomas L. Petersen

Robert S. Massey

Joe S. Sandoval

John E. Baran

CTR-4

Robert S. Dike

Garrett A. Barnes

John D. Galbraith

Robert Benavidez

Robert F. Gribble

Tom Dominguez

Charles F. Hammer

Phill1p A. Freeman

Jimmy G. Me1ton

Lou S. Schrank

Ra1ph W. Kewish

Alex M. Velasquez

Kurt F. Schoenberg

Jim A. Meyer 
CTR-7

C. Jerald Buchenauer

James N. Downing

Edward M. Little

Ke1th S. Thomas

Robert G. Watt

Pau1 G. Weber

Peter R. Forman

Ronald W. Wilkins

Kenneth A. Klare

George I. Chandler

Franz C. Jahoda

Kenneth B. Freese

Marvin Bausman
Jose A. Garcia

Raymond Lopez

Camilo E. Medina

Michael D. Pacheco

Jose D. Sandoval

Emmanue1 K. Spanos

CTR-8

Larry Caud 111

Jose F. Trujillo

Charles Roger Hall

Robert M. Carpenter

Richard W. Howell

J. D. Smith

Terry D. Langham

Martin A. Leif 